\title{
Kognitive \\ Verarbeitungsprozesse beim kausalen Urteilen und Entscheiden
}

\author{
Dissertation \\ zur Erlangung des mathematisch-naturwissenschaftlichen Doktorgrades \\ „Doctor rerum naturalium“ \\ der Georg-August-Universität Göttingen
}

vorgelegt von

Stefan Mangold

aus Fritzlar

Göttingen, im Juni 2012 
Referent: PD Dr. York Hagmayer

Korreferent: Prof. Dr. Hannes Rakoczy

Tag der mündlichen Prüfung: 23.07.2012 

Inhaltsverzeichnis

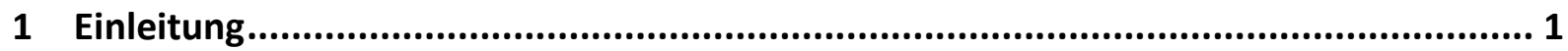

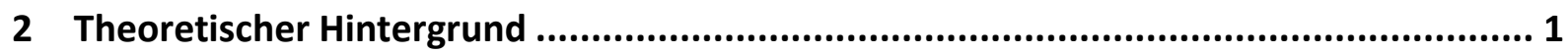

2.1 Kausales Denken als eigenständige Denkform: Charakteristika kausaler Beziehungen .................................................................................................... 1

2.1.1 Gemeinsames Auftreten von Ursache und Effekt............................................... 5

2.1.2 Zeitliche Abfolge von Ursache und Effekt ................................................................ 7

2.1.3 Räumliche und zeitliche Nähe von Ursache und Effekt (Kontiguität)........................ 8

2.1.4 Gerichtetheit der Beziehung zwischen Ursache und Effekt.................................. 11

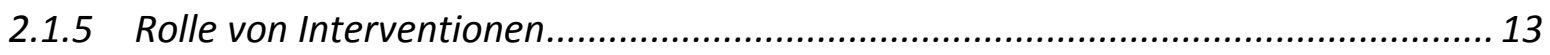

2.1.6 Mechanismen zwischen Ursachen und Effekten ................................................ 15

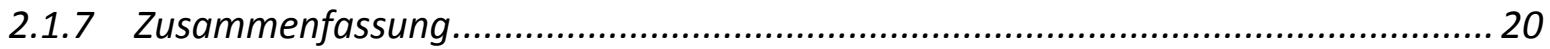

2.2 Prozesse kausalen Denkens in Zwei-Prozess-Theorien der Urteils- und Entscheidungsforschung........................................................................ 20

2.2.1 Ursprünge der modernen 2-Prozess-Theorien........................................................ 21

2.2.2 Gemeinsamkeiten verschiedener Zwei-Prozess-Theorien ..................................... 23

2.3 Kausales Denken und Zwei-Prozess-Theorien ................................................. 26

2.3.1 Verarbeitung kausaler Informationen als Typ 1-Prozess ........................................ 28

2.3.2 Verarbeitung kausaler Informationen als Typ 2-Prozess ....................................... 34

2.4 Berücksichtigung kognitiver Prozesse in kausalen Theorien .............................. 41

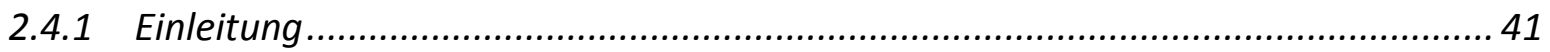

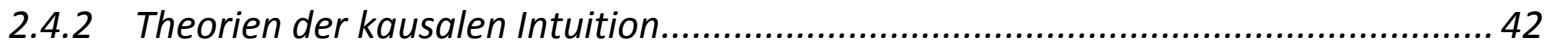

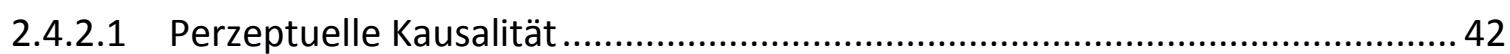

2.4.2.2 Urteilsverzerrungen.................................................................................. 46

2.4.2.3 Einflüsse kausalen Vorwissens auf Kategorisierungsurteile............................. 49

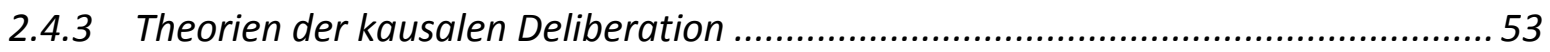

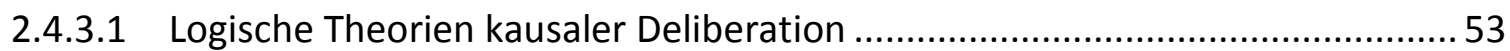

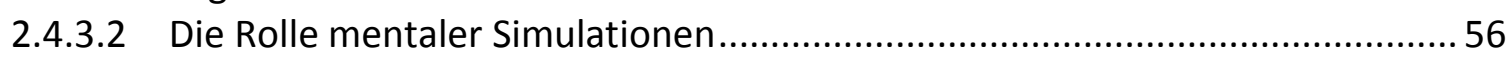

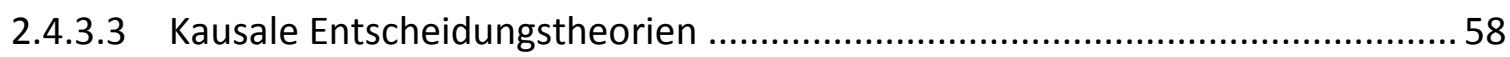

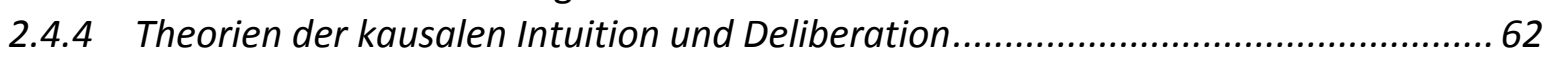

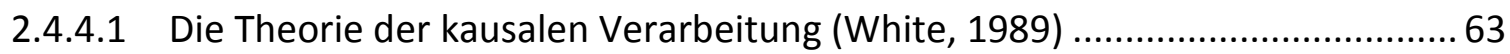

2.4.4.2 Der Zwei-Prozess-Ansatz von Fugelsang und Thompson (2003) ......................66 66

2.4.4.3 Der Zwei-Prozess-Ansatz von Verschueren, Schaeken und d'Ydewalle (2005) 67

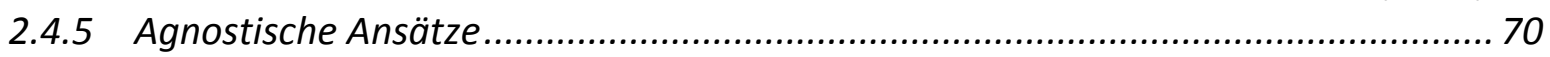

2.4.5.1 Das Modell der Kausalattribution von Einhorn und Hogarth (1986) ................ 71

2.4.5.2 Das Force Dynamics Model von Wolff (2007) ............................................... 75

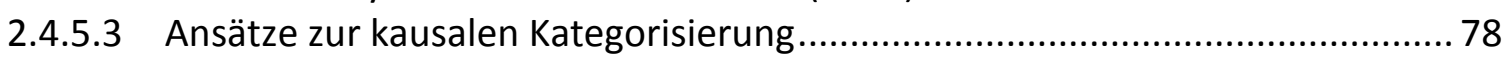

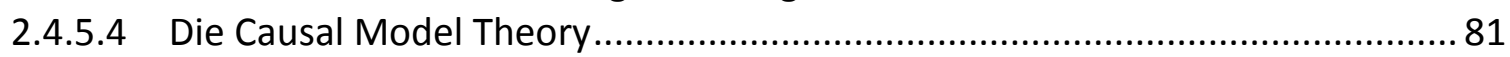

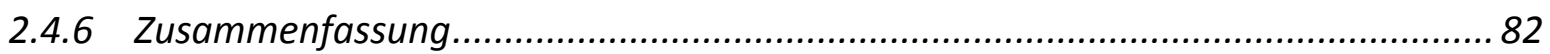

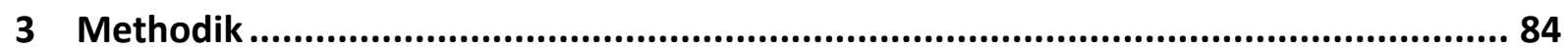

3.1 Wahl der experimentellen Bedingungen .................................................. 85

3.2 Die Theorie des unbewussten Denkens (Dijksterhuis \& Nordgren, 2006) ............... 90 


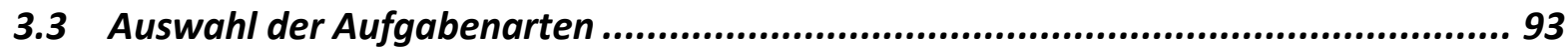

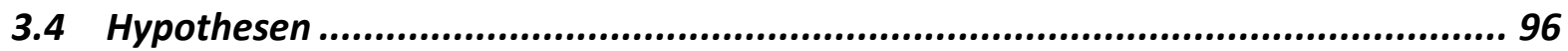

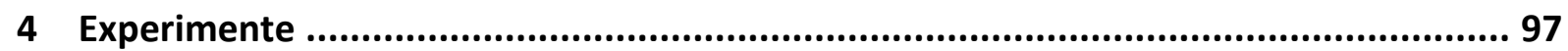

4.1 Überblick über die durchgeführten Experimente und allgemeiner Ablauf.............. 97

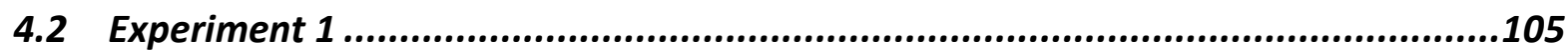

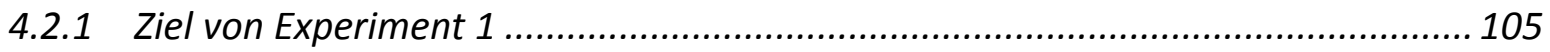

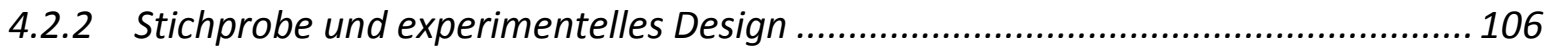

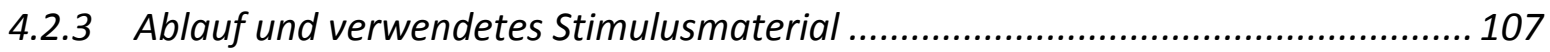

4.2.3.1 Instruktionen Kausalsystem Kategorisierungsaufgaben ............................... 108

4.2.3.2 Instruktionen Kausalsystem Interventionsaufgaben................................... 113

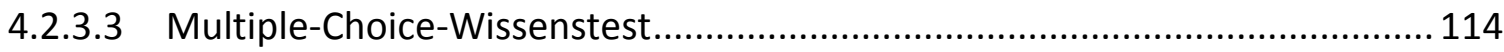

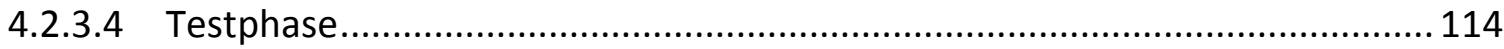

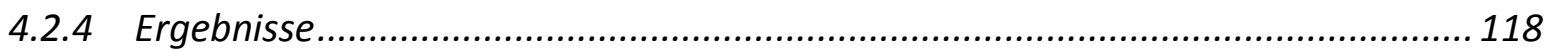

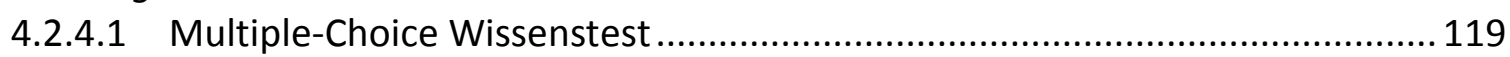

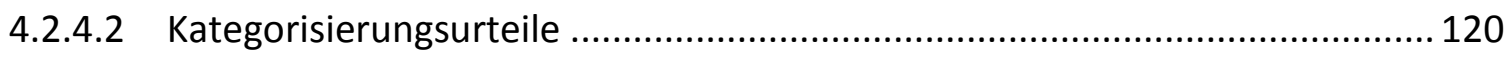

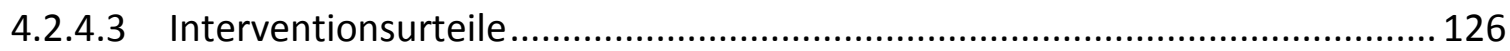

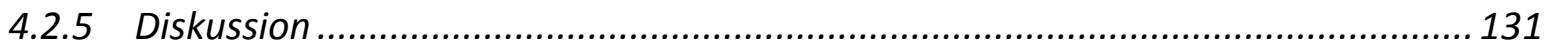

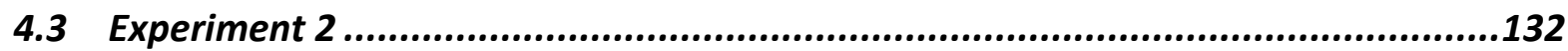

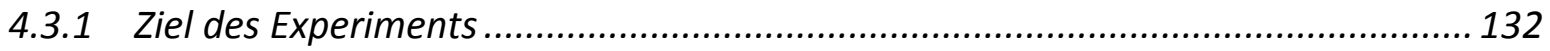

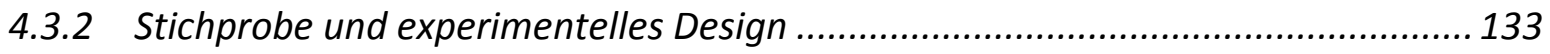

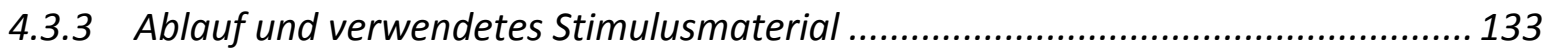

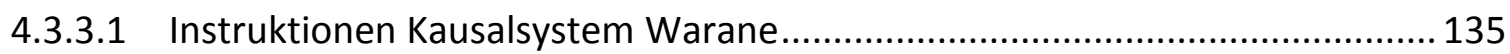

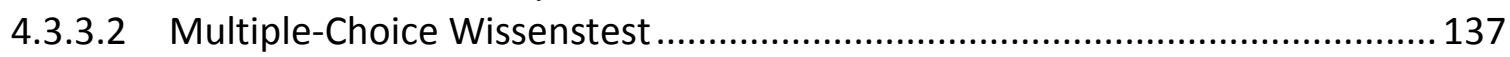

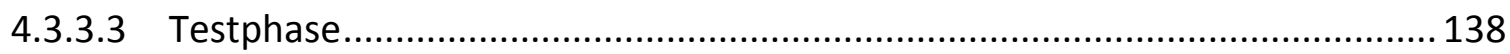

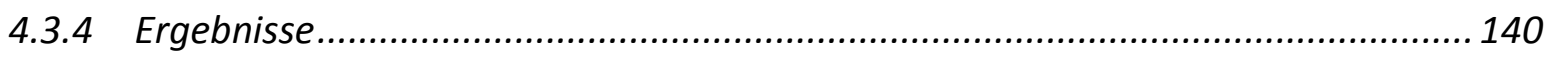

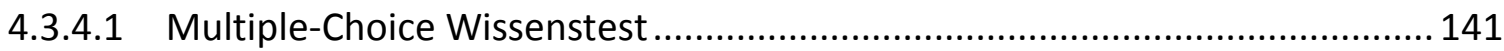

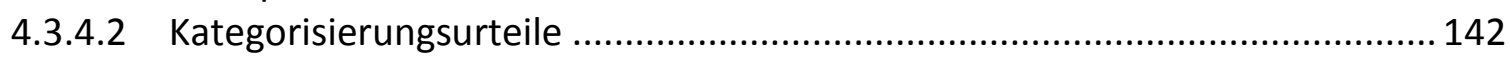

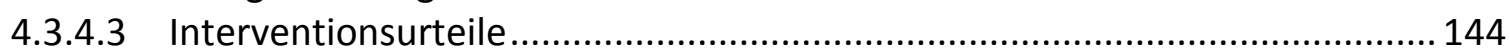

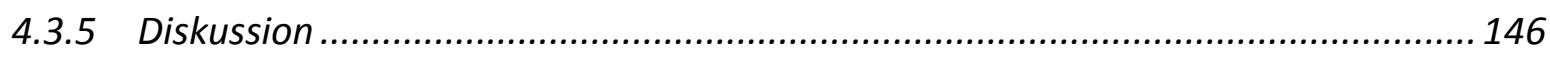

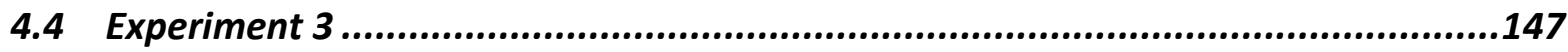

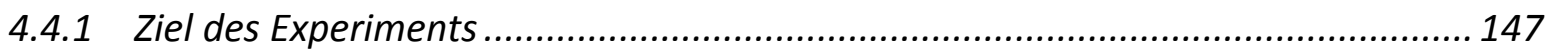

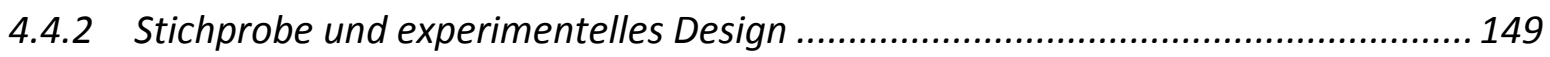

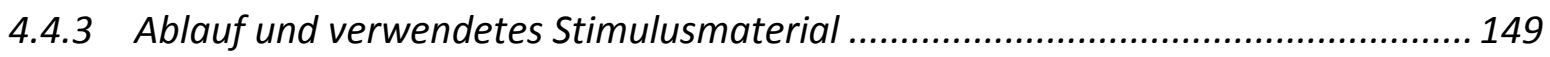

4.4.3.1 Instruktionen Kausalsystem Post-Embitterment-Disorder ........................... 150

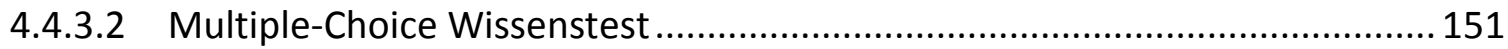

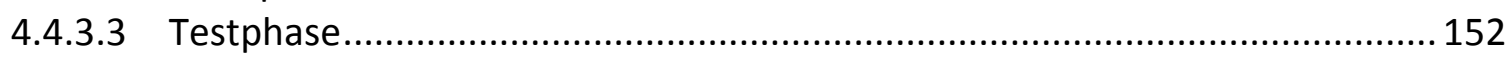

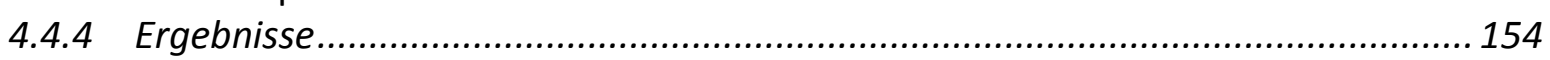

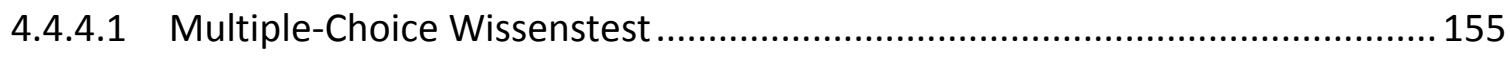

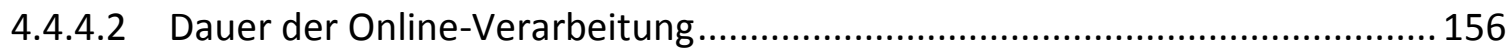

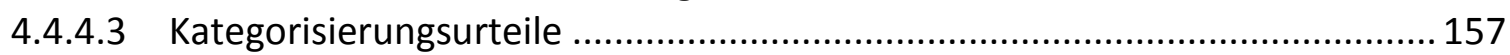

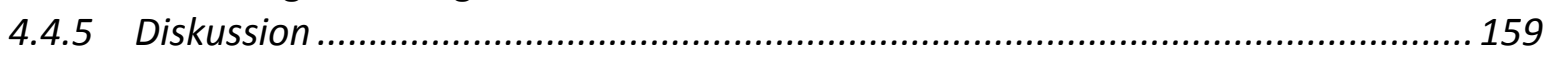

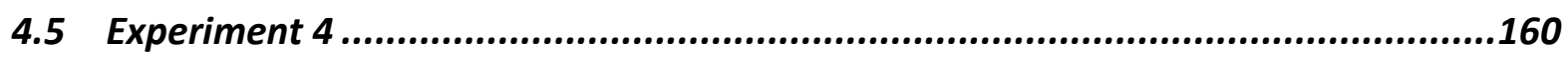

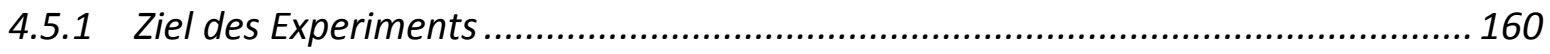

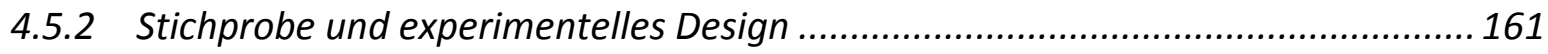

4.5.3 Ablauf und verwendetes Stimulusmaterial ........................................................ 161 


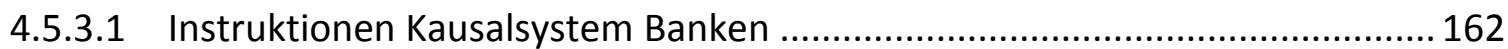

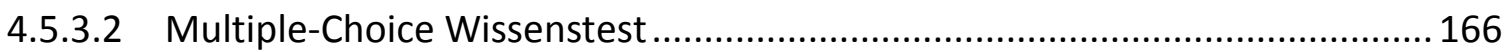

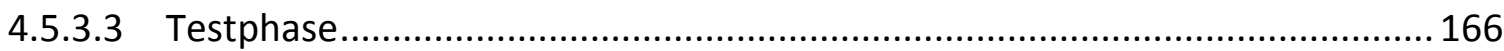

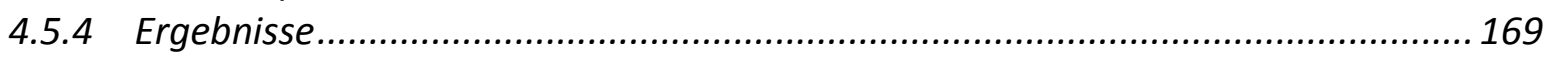

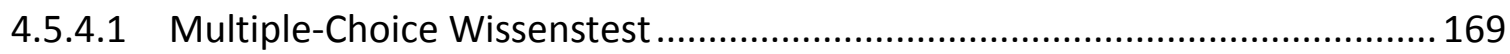

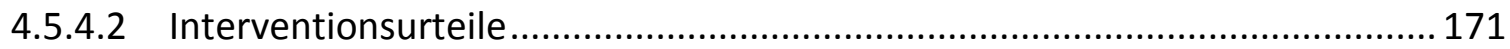

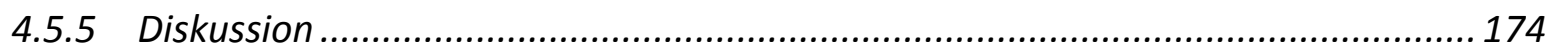

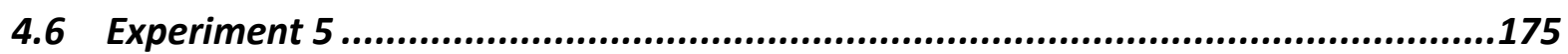

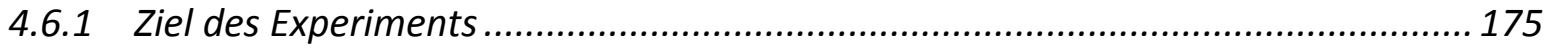

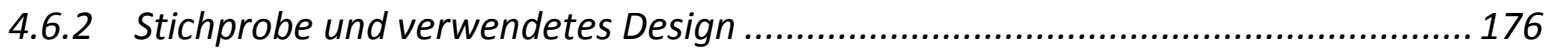

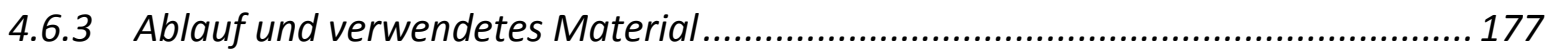

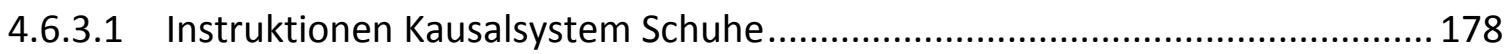

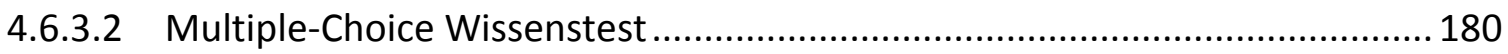

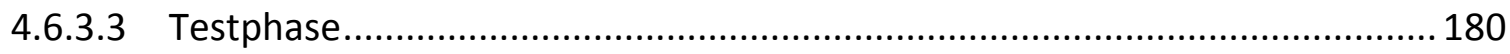

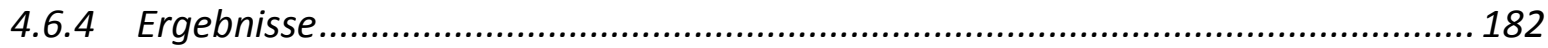

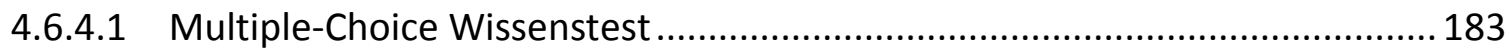

4.6.4.2 Vorhersagen auf Grundlage kausaler Mechanismen .................................... 184

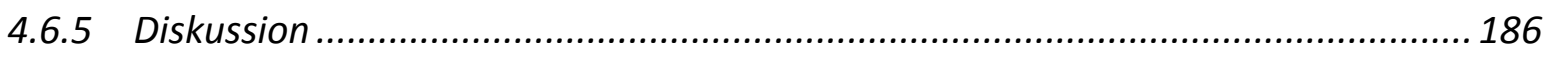

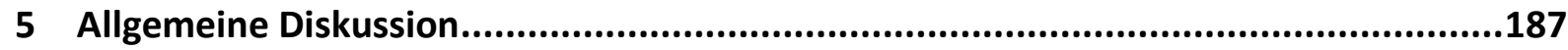

5.1 Bewertung von Hypothese 1 anhand der Ergebnisse der Kategorisierungsexperimente

5.2 Bewertung von Hypothese 2 anhand der Ergebnisse der Interventionsexperimente

5.3 Unbewusstes Denken bei Kausalurteilen? ...................................................202

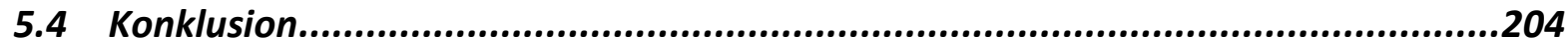

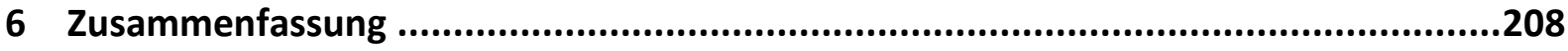

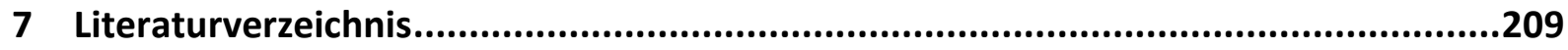

Anhang A - Multiple-Choice-Fragen und -Antworten in Experiment 1 ..........................219

Anhang B - Zusätzliche Varianzanalytische Ergebnisse in Experiment 1 ........................222

Anhang C - Multiple-Choice-Fragen und Antworten in Experiment 2 .............................223

Anhang D - Multiple-Choice-Fragen und Antworten in Experiment 3 .........................226

Anhang E - Instruktionen für Balancierungsbedingung 2 in Experiment $4 \ldots \ldots \ldots \ldots \ldots \ldots \ldots . . . . . . . . .228$

Anhang F - Multiple-Choice-Fragen und Antworten in Experiment 4 .............................230

Anhang G - Instruktionen für Balancierungsbedingung 2 in Experiment 5 .....................235

Anhang H - Multiple-Choice-Fragen und Antworten in Experiment 5 ...........................237

Anhang I - Kontraste zwischen den Bedingungen in Experiment 5.............................240 


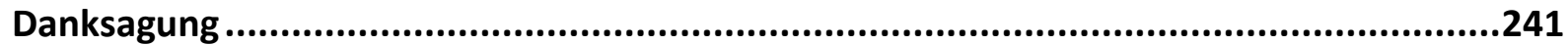

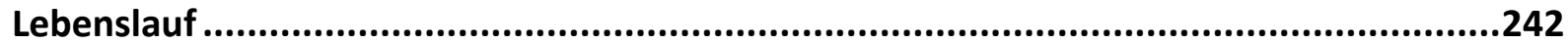




\section{Einleitung}

Innerhalb der Kognitionspsychologie gibt es lebhafte Debatten über die Art und Anzahl der Prozesse, die dem Denken zu Grunde liegen. In der vorliegenden Arbeit soll es um die Frage gehen, welche kognitiven Prozesse beim kausalen Denken grundlegend sind. Dazu soll zunächst geklärt werden, ob kausales Denken eine eigenständige Form des Denkens ist. Nach der positiven Beantwortung dieser Frage werden in den darauf folgenden Abschnitten verschiedene theoretische Ansätze des kausalen Denkens im Hinblick auf die angenommenen kognitiven Prozesse analysiert. Im Vordergrund wird dabei die Unterscheidung von intuitiven Typ 1-Prozessen und deliberativen Typ 2-Prozessen stehen. Wie die Analyse zeigen wird, vertreten manche theoretische Modelle die Ansicht, dass kausales Denken auf Typ 1Prozessen beruht, während es von anderen Typ 2-Prozessen zugeordnet wird. Eine dritte Gruppe von Ansätzen versteht kausales Denken als eine Kombination von Typ 1- und Typ 2Prozessen, eine vierte sieht es hingegen als agnostisch an. Zur Untersuchung der dem kausalem Denken zu Grunde liegenden Prozesse wird im zweiten Teil dieser Arbeit ein neues Untersuchungsparadigma entwickelt. Es werden fünf experimentelle Studien vorgestellt, deren Ergebnisse im letzten Teil zusammenfassend diskutiert werden.

\section{Theoretischer Hintergrund}

\subsection{Kausales Denken als eigenständige Denkform: Charakteristika kausaler Beziehungen}

Eine der für die vorliegende Arbeit wichtige Diskussion in der Kognitionspsychologie beschäftigt sich mit der Frage, ob kausales Denken als eigenständige Form des Denkens verstanden werden kann oder lediglich ein Spezialfall allgemeinerer Denkformen ist. Im folgenden Abschnitt soll dieser Frage nachgegangen werden. Dazu werden die besonderen Charakteristika kausaler Beziehungen betrachtet und verschiedene Theorien auf ihre Eignung diese Besonderheiten zu erfassen untersucht. Die betrachteten Theorien lassen sich dabei grob unterteilen in diejenigen, die kausales Denken als Spezialfall einer domänenübergreifenden, allgemeinen Fähigkeit zu Denken ansehen und diejenigen, die aus den Eigenschaften kausaler Beziehungen die Notwendigkeit ableiten, kausales Denken als eigenständige Denkform anzunehmen. Es wird gezeigt, dass die zweite Art von Theorien besser geeignet ist, die Eigenheiten kausalen Denkens zu erklären. Die Eigenständigkeit kausalen Denkens bildet den Ausgangspunkt für die in den Abschnitten 2.3 und 2.4 vorgenommene Analyse verschiedener 
theoretischer Ansätze hinsichtlich der kognitiven Prozesse, die dem Umgang mit kausalen Phänomenen zu Grunde liegen.

Im Fokus der vorliegenden Arbeit soll nicht die Frage nach der Existenz von Kausalität in der Welt stehen, sondern die Frage, ob und wie Menschen Ereignisse kausal repräsentieren und für ihre Entscheidungen nutzen können. Zur Beantwortung dieser Frage ist es wichtig zu wissen, ob Menschen die besonderen Charakteristika kausaler Beziehungen beim kausalen Denken berücksichtigen oder ob ihre Repräsentationen ohne sie gebildet werden. Darüber hinaus werden verschiedene Theorien im Hinblick auf ihre Berücksichtigung der von Menschen repräsentierten Charakteristika kausaler Beziehungen untersucht, um ihre Eignung für Entscheidungen auf der Grundlage kausalen Wissens zu prüfen.

David Hume (1739/1978) gilt als klassischer Vertreter der Ansicht, dass eine Verbindung zwischen einer Ursache und ihrem Effekt nicht notwendigerweise in der Welt existieren muss, sondern vielmehr als eine aus gewohnheitsmäßigen Beobachtungen der Kovariation zweier Ereignisse abgeleitete Erwartung über den Ablauf von Ereignissen angesehen werden kann. Zusammen mit der Beobachtung einer festgelegten zeitlichen Abfolge, bei der die Ursache dem Effekt stets vorausgeht, sowie einer räumlichen und zeitlichen Nähe (Kontiguität) von Ursache und Wirkung wird nach Hume die Empfindung von Kausalität erzeugt. Neben diesen Kriterien gibt es nach Ansicht Humes keine weiteren empirisch belegbaren Eigenschaften kausaler Beziehungen, die auf die tatsächliche Existenz versteckter kausaler Kräfte hinweisen. Ausgehend von den Erkenntnissen Humes entwickelten sich bis in die Gegenwart Theorien, deren Fokus auf der Assoziation von Veränderungen in zeitlich vorausgehenden Variablen mit Veränderungen in anderen, zeitlich nachfolgenden Variablen liegt (vgl. Waldmann \& Hagmayer, in Druck). Somit betrachten diese Theorien vor allem die Kovariation zwischen Ereignissen als Grundlage kausaler Inferenz. Diese Theorien werden im weiteren Verlauf dieser Arbeit unter dem Terminus ,kovariationsbasierte Ansätze ‘ zusammengefasst. Eine weitere Klasse von nicht auf dem Konzept von Kausalität basierenden Ansätzen bilden die logischen Theorien (z.B. die Mental Model Theory of Causal Meaning and Reasoning (Goldvarg \& Johnson-Laird, 2001). Im Zentrum dieser Theorie steht die Annahme, dass Menschen Aussagen über kausale Beziehungen mit Hilfe von auf Propositionen basierenden mentalen Modellen repräsentieren und kausale Schlüsse auf Grundlage dieser Modelle ziehen. Wie die kovariationsbasierten Ansätze gehen auch die logischen Theorien davon aus, das kausales Denken lediglich einen 
Spezialfall eines allgemeineren, domänenübergreifenden Denkprozesses darstellt (vgl. Waldmann \& Hagmayer, in Druck).

Eine andere Sichtweise auf Kausalität liefern diejenigen Theorien (im Folgenden unter dem Begriff ,kausale Theorien‘ subsummiert), deren Fokus auf der Erklärung der durch kausale Ereignisse herbeigeführten Prozesse und Mechanismen liegt (vgl. Waldmann und Hagmayer, in Druck). Kausale Mechanismen bilden dabei den Kern der kausalen Beziehung, indem sie Ursachen und die von ihr hervorgerufenen Effekte verbinden. Einige philosophische (z.B. Glennan, 2009; Machamer, Darden \& Craver, 2000) wie auch psychologische Theorien (z.B. White, 1989; Ahn, Kalish, Medin und Gelman, 1995) sehen Wissen um kausale Mechanismen als grundlegend für die Inferenz von Kausalität an. Daneben beschäftigen sich einige Ansätze mit dem Verhältnis von Mechanismus-Wissen und kovariationbasierten Wissen (z.B. Ahn et al., 1995; Buehner, 2005; Fugelsang \& Thompson, 2003; Hagmayer \& Waldmann, 2002). Andere Ansätze legen ihren Fokus wiederrum auf die Beziehungen zwischen zwei konkreten Ereignissen (z.B. White, 2006a, 2009; Wolff, 2007) und befinden sich somit auf einer spezifischeren Betrachtungsebene, auf der keine kovariationsbasierten Informationen verfügbar sind. Schließlich gibt es noch Theorien kausaler Bayes-Netze, die eine Integration von Wissen über einzelne Kausalbeziehungen zwischen Ereignissen und kovariationsbasierten

Informationen mittels kausaler Bayes-Netze vornehmen (z.B. Pearl, 1988, 2009; Spirtes, Glymour \& Scheines, 2000). Auf Grundlage dieser komputationalen Modelle entwickelten sich auch eine Reihe psychologischer Ansätze, insbesondere auch die Causal Model Theory (Waldmann, 1996; Waldmann \& Holyoak, 1992; Waldmann, Holyoak \& Fratianne, 1995; für einen Überblick anderer psychologischer Ansätze s. Gopnik, Glymour, Sobel, Schulz, Kushnir \& Danks, 2004; Sloman, 2005). Diese Theorie geht davon aus, dass Menschen kausale Zusammenhänge als Modelle repräsentieren und für Urteile und Vorhersagen nutzen. Dabei können die kausalen Modelle auch aus mehreren Variablen und Relationen bestehen. Kausale Bayes Netze (vgl. Glymour, 2001, Pearl, 2009) stellen eine Möglichkeit dar, kausale Zusammenhänge formal darzustellen (vgl. Abbildung 1 für eine graphische Darstellung verschiedener kausaler Strukturen). Dabei werden die kausal relevanten Variablen durch Knoten repräsentiert. Die kausalen Beziehungen zwischen den Variablen werden als Pfeile dargestellt, die von einer Ursache zu ihrem Effekt zeigen. Eine wichtige Eigenschaft kausaler Bayes Netze liegt in ihrem Vermögen, die Konsequenzen von 
Veränderungen der durch die Variablen abgebildeten Entitäten oder durch externe Einwirkungen abzubilden.

a)

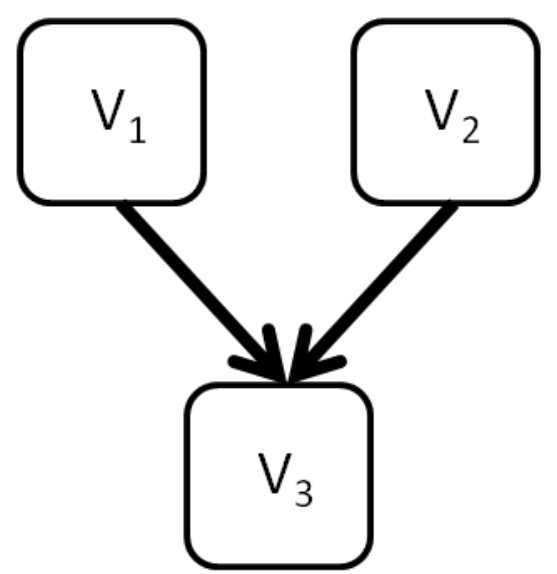

b)

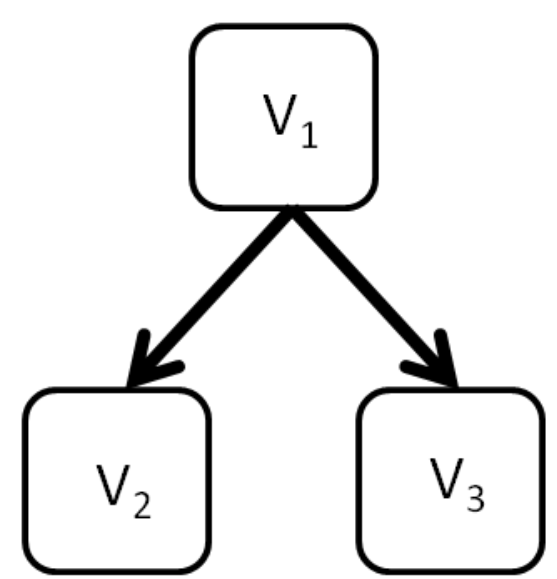

c)

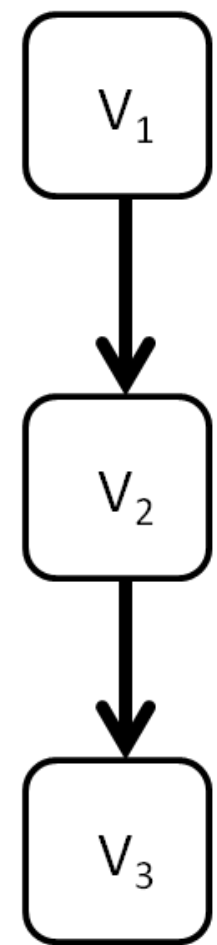

Abbildung 1: Graphische Darstellung von drei einfachen kausalen Strukturen. a) Gemeinsamer Effekt Modell: $V_{1}$ und/oder $V_{2}$ verursachen $V_{3}$ werden. b) Gemeinsame Ursache Modell: $V_{1}$ verursacht sowohl $V_{2}$ als auch $V_{3}$. c) Kausale Kette: $V_{1}$ verursacht $V_{2}, V_{2}$ verursacht $V_{3}$.

Eine weitere Gruppe von Theorien kausaler Inferenz kann zwischen den kovarianzbasierten und den kausalen Theorien eingeordnet werden, da sie gewissermaßen einen Übergang darstellt (vgl. Waldmann \& Hagmayer, in Druck). Prominente Vertreter dieser komputationalen Erklärungen kausalen Denkens sind die PowerPC-Theorie von Cheng (1997) und der Causal Support-Ansatz von Griffiths und Tenenbaum (2005). Sie berücksichtigen die Tatsache, dass kausale Zusammenhänge nicht immer hinreichend mittels kovariationsbasierten Informationen abbildbar sind und versuchen dies durch die Berücksichtigung einiger Charakteristika kausaler Beziehungen zu kompensieren. Zudem gehen sie davon aus, dass Menschen rational Schließen, wann immer dies möglich ist. Beide Theorien beschäftigen sich mit der Schätzung von Parametern, die den Zusammenhang zwischen einer Ursache und einem von ihr hervorgerufenen Effekt charakterisieren. Dabei liefert die PowerPC-Theorie Punktschätzungen für die Stärke der Beziehung zwischen Ursache und Effekt, während die Causal Support-Theorie Schätzungen von Parameterverteilungen für die Existenz einer Beziehung zwischen Ursache und Effekt angibt. 
Die verschiedenen hier kurz erwähnten Theorien unterscheiden sich vor allem im Hinblick auf die von ihnen berücksichtigten Charakteristika kausaler Beziehungen. Inwiefern diese Charakteristika erlauben zwischen den verschiedenen Theorien zu differenzieren, es ermögliche Schlüsse über die Existenz kausaler Beziehungen zu ziehen und beim Urteilen und Entscheiden mittels kausaler Beziehungen eine Rolle spielen, soll daher im folgenden Abschnitt näher erläutert werden. Dazu werden neben den auf Hume (1739/1978) zurückgehenden Charakteristika gemeinsames Auftreten (Kontingenz), zeitliche Abfolge und räumlich-zeitliche Nähe (Kontiguität) von Ursache und Effekt auch das Wissen um die Existenz kausaler Mechanismen sowie die Gerichtetheit der Beziehung von Ursache und Effekt inkl. der Rolle von Interventionen beleuchtet.

\subsubsection{Gemeinsames Auftreten von Ursache und Effekt}

Kontingenz stellt einen der wichtigsten Indikatoren kausaler Beziehungen dar. Auch das alltägliche Verständnis von Kausalität ist nahezu untrennbar mit der Annahme eines gemeinsamen Auftretens von Ursachen mit ihren Effekten verbunden. Darüber hinaus erscheint eine Beziehung, bei der die Ursache häufig von ihrem Effekt gefolgt wird stärker als eine Beziehung, bei der dies nur selten der Fall ist. Eine Vielzahl empirischer und theoretischer Studien haben sich mit diesem auf Hume (1739/1978) zurückgehende Charakteristikum kausaler Beziehungen auseinandergesetzt. Die Klasse der kovariationsbasierten Ansätze sieht Informationen über die Kovariation von Ursache und Effekt als den wichtigsten empirischen Indikator für die Interpretation einer Beziehung als kausal an. Diese Klasse beinhaltet neben den assoziativen noch die probabilistischen Theorien kausaler Inferenz. Den assoziativen Ansätzen zufolge wird eine Beziehung zwischen zwei Variablen (die im Rahmen dieser Theorien nicht als Ursache und Effekt, sondern als Cue und Outcome bezeichnet werden) aus einer Kombination von kovariationsbasierten Informationen und der Annahme einer zeitlichen Priorität des cues Ursache gegenüber dem Outcome inferiert werden.

Eine weitere Klasse von Theorien, die Kausalität vornehmlich aus kovariationsbasierten Informationen herleiten, bilden die probabilistischen Theorien (Eells, 1991; Salmon, 1984; Spirtes, Glymour \& Scheines, 2000; Suppes, 1970, 1974). Bei ihnen steht die Häufigkeit der verschiedenen Kombinationsmöglichkeiten der An- bzw. Abwesenheit zweier Variablen im Zentrum der Inferenz kausaler Zusammenhänge. Probabilistische Theorien interpretieren die Häufigkeitsinformationen i.d.R. im Sinne einer gerichteten Beziehung von Ursache zu Effekt. Allerdings kann Kausalität nach Waldmann 
und Hagmayer (in Druck) nicht als intrinsischer Bestandteil probabilistischer Theorien angesehen werden, da es ihnen vornehmlich darum geht, welche Arten von Kovariationsmetriken Menschen nutzen, um die Stärke von Ursache-Wirkungs-Relationen zu bestimmen. Ein bekanntes Maß hierfür stellt die Delta-P-Regel dar. Sie besagt, dass sich die Stärke eines (Kausal-)Zusammenhangs aus der Differenz der Wahrscheinlichkeit des Auftretens eines Effektes bei Anwesenheit der Ursache $(\mathrm{P}(\mathrm{E} \mid \mathrm{C}))$ und der Wahrscheinlichkeit des Auftretens des Effektes bei Abwesenheit der Ursache $(\mathrm{P}(\mathrm{E} \mid \sim \mathrm{C})$ ) ergibt (Jenkins \& Ward, 1965 zit. n. Waldmann \& Hagmayer, in Druck)

$$
\Delta \mathrm{P}=\mathrm{P}(\mathrm{e} \mid \mathrm{c})-\mathrm{P}(\mathrm{e} \mid \sim \mathrm{c})
$$

Die logischen Theorien betrachten kausales Denken als Spezialfall des deduktiven Schließens, das eine allgemeinen, domänenübergreifenden Denkfähigkeit darstellt, und sehen es deshalb wie die kovariationsbasierten Ansätze nicht als eigenständige Denkform an (vgl. Waldmann und Hagmayer, in Druck). In den logischen Theorien werden kausale Beziehungen mit den Gesetzen der Aussagenlogik repräsentiert. So lässt sich diesen Theorien zufolge beispielsweise aus den Prämissen „Wenn Ursache C, dann Effekt E“ und „Ursache C“, die Konklusion „Effekt E“ ableiten (Modus ponens). Das gemeinsame Auftreten von Ereignissen (z.B. C und E, nicht-C und nicht-E) ermöglicht diesen Theorien zufolge einen Schluss auf das Vorliegen bzw. Nicht-Vorliegen kausaler Beziehungen. Daher spielen kovariationsbasierte Informationen bei den logischen Theorien eine wichtige Rolle.

Kovariationsbasierte Informationen werden jedoch auch von denjenigen Theorien berücksichtigt, die davon ausgehen, dass Kausalität nicht nur einen Spezialfall eines allgemeinen, domänenübergreifenden Denkprozesses darstellt (vgl. Waldmann \& Hagmayer, in Druck). Sie nehmen hier allerdings eine weniger zentrale Rolle ein und bilden nur einen von mehreren Indikatoren von Kausalität. So sieht z.B. die Power PC Theorie (Cheng, 1997) die Stärke einer kausalen Beziehung zwischen zwei Variablen als eine theoretische Größe an, die auf Grundlage von mit Vorwissen angereicherten Kovariationsinformationen inferiert werden kann. Die einzige Ausnahme bilden die Theorien kausaler Kräfte (z.B. White, 2006a, 2009; Wolff, 2007), die eine Betrachtung einzelner, konkreter Ereignisse zum Gegenstand haben und daher nicht auf kovariationsbasierte Informationen zurückgreifen können.

Der Einfluss kausalen Vorwissens auf die Bewertung von kovariationsbasierten Informationen wurde u.a. von Waldmann und Hagmayer (2001)untersucht. Es konnte gezeigt werden, dass bei der Bewertung der Kausalstärke einer instruierten Ursache-Effekt-Beziehung 
der Wert einer dritten Variablen nur dann von Bedeutung für das Urteil war, wenn diese als mögliche weitere Ursache des Effektes angesehen wurde. Hingegen spielte die dritte Variable keine Rolle, wenn sie ein weiterer Effekt der Ursache-bzw. der Effekt-Variablen war oder die Ursache der Ursache-Variablen war.

\subsubsection{Zeitliche Abfolge von Ursache und Effekt}

Ein weiteres, ebenfalls auf Hume (1739/1978) zurück gehendes Charakteristikum kausaler Beziehungen ist die zeitliche Reihenfolge von Ursache und Effekt. Ursachen gehen ihren Effekten stets voraus. Dieses Charakteristikum kausaler Beziehungen ist somit geeignet um Variablen als potenzielle Ursachen eines Effektes auszuschließen, wenn diese zeitlich nach dem Effekt auftreten. Andererseits ist die zeitliche Abfolge jedoch nicht hinreichend, um eine Variable eindeutig als Ursache eines Effektes zu identifizieren, da die Möglichkeit einer Konfundierung durch eine weitere Variable besteht, die in Wirklichkeit die Ursache sowohl des Effektes als auch der aufgrund der zeitlichen Abfolge möglichen potenziellen Ursache ist (vgl. Lagnado, Waldmann, Hagmayer \& Sloman, 2007).

Wie Wolff (2007) kritisch anmerkt, gibt es jedoch auch eine Reihe von kausalen Ereignissen, bei denen das Auftreten der Ursache vor dem Effekt nicht zwingend notwendig sei. So setzt die Wirkung physikalischer Kräfte wie z.B. der Gravitation ohne zeitlichen Verzug ein, Ursache und Effekt treten hier simultan auf. Des Weiteren können in statischen Situationen, d.h. in Situationen, in denen nichts passiert (wie z.B. „Der Nagel bewirkt, dass das Bild an der Wand hängt"), keine Aussagen über zeitliche Abfolgen von Ursache und Effekt getroffen werden obwohl auch hier Kausalattributionen möglich sind. Somit gibt es Situationen, in denen nicht von einer Sequenz Ursache-Effekt ausgegangen werden kann. Es ist jedoch weiterhin nicht möglich, dass ein Effekt vor seiner Ursache eintritt, so dass die Annahme der zeitlichen Priorität der Ursache mit der Einschränkung eines möglichen gleichzeitigen Auftretens von Ursache und Effekt aufrecht erhalten werden kann.

Der Bedeutung des Charakteristikums der zeitlichen Abfolge von Ursache und Effekt wird auch dadurch Rechnung getragen, dass sie in alle betrachteten Theorien kausaler Inferenz einfließt. So berücksichtig die logische Mental Model Theory of Causal Meaning and Reasoning (Goldvarg \& Johnson-Laird, 2001) dass Ursachen ihren Effekten vorangehen. Sie geht davon aus, dass die zeitliche Abfolge von Ursache und Effekt in den mentalen Modellen, die die kausalen Beziehungen repräsentieren, berücksichtigt werden. 
In den assoziativen Theorien wird die zeitliche Priorität des Cues vor dem Outcome berücksichtigt. Dabei muss jedoch beachtet werden, dass die zeitliche Abfolge von Cue und Outcome nicht immer der zeitlichen Abfolge von Ursache und Effekt entspricht, da einige assoziative Theorien die wahrgenommene Abfolge der Variablen beim Lernen von Beziehungen als den entscheidenden Hinweis für die Repräsentation einer Relation zwischen Cue und Outcome ansehen (Lagnado et al., 2007). Es ist jedoch auch möglich, dass die Wahrnehmung von Ursache und Effekt zeitversetzt erfolgt, so dass der Effekt vor der Ursache wahrgenommen wird. Dies ist beispielsweise auch in Experimenten der Fall, in denen ausgehend vom Vorliegen bestimmter Symptome (Effekte) auf deren Ursache(n) geschlossen werden soll (z.B. Waldmann \& Holyoak, 1992). In diesen Fällen würden assoziative Theorien die Rollen von Ursache (Cue) und Effekt (Outcome) vertauschen und die Relation somit nicht korrekt wiedergeben. Waldmann und Holyoak konnten zeigen, dass Versuchspersonen mit entsprechendem kausalen Vorwissen sensitiv für die kausale Abfolge von Ursache und Effekt sind, da sie trotz der zeitversetzten Wahrnehmung (Effekt vor Ursache) weiterhin auf Grundlage der Kausalrelation urteilten. Des Weiteren werden Informationen über Variablen oftmals symbolisch (z.B. in Textform) präsentiert (Lagnado et al., 2007), wodurch die zeitliche Abfolge von Ursache und Effekt nicht als feststehend angesehen werden kann. Daher hat nach Ansicht von Vertretern kausaler Theorien das Charakteristikum der zeitlichen Abfolge von Ursache und Effekt in rein kovariationsbasierten Theorien des kausalen Denkens keinen wesentlichen Stellenwert (vgl. Greville \& Buehner, 2010; Waldmann \& Hagmayer, in Druck).

\subsubsection{Räumliche und zeitliche Nähe von Ursache und Effekt (Kontiguität)}

Eng verwandt mit dem Charakteristikum der zeitlichen Abfolge ist die Bedeutung der räumlichen und zeitlichen Nähe (Kontiguität) von Ursache und Effekt bei der Zuschreibung einer kausalen Beziehung. Diese beiden Charakteristika kausaler Beziehungen werden im Rahmen dieser Arbeit unterschieden, da die zeitliche Abfolge von Ursache und Effekt und der Abstand zwischen ihnen verschiedene Implikationen für Kausalitätszuschreibungen haben. Während die zeitliche Abfolge wie bereits erläutert ein Ausschlusskriterium für potenzielle Ursachen darstellt, kann der Abstand zwischen Ursache und Effekt einen Einfluss auf die Bewertung der Stärke der kausalen Verbindung ausüben.

Kontiguität war in der Vergangenheit bereits oft ein Gegenstand empirischer Forschung (vgl. Buehner, 2005, für eine Übersicht). Dabei wurde vor allem die Länge des zeitlichen Intervalls zwischen Ursache und Effekt als Einflussfaktor auf die wahrgenommene 
Stärke eines kausalen Zusammenhangs untersucht. Wie Shanks, Pearson und Dickinson (1989, zit n. Lagnado et al, 2007) zeigen konnten wird eine Kausalrelation mit zunehmender Länge des Intervalls als weniger stark bewertet, es sei denn die Verzögerung kann mittels Vorwissen begründet werden (z.B. Buehner \& May, 2002; Buehner, 2005; Hagmayer und Waldmann, 2002). Kritik an der Annahme der Bedeutung der Länge des Ursache-EffektZeitintervalls für die Bewertung der Stärke einer Kausalbeziehung leitet Buehner (2005) aus der Rate Estimation Theory von Gallistel und Gibbon (2000) ab, die einen kontiguitätsbasierten Ansatz konditionalen Lernens liefert. Der Theorie zufolge basiert konditionales Verhalten nicht auf der Wahrscheinlichkeit von Verstärkungen, sondern auf dem Zeitintervall zwischen Verstärkung und konditioniertem Stimulus. Daher gehen Gallistel und Gibbon davon aus, dass die für das gezeigte Verhalten relevante Variable die Rate der Verstärkungen ist. Wichtig für die vorliegende Arbeit ist das Phänomen der sog. timescale invariace, das auftritt, wenn nach Veränderung sämtlicher Zeitintervalle in einem Experiment durch Multiplikation mit einem konstanten Skalierungsfaktor das Konditionierungsergebnis unverändert bleibt. Gallistel und Gibbon konnten die timescale invariance in einer Vielzahl von Konditionierungsbedingungen nachweisen. Buehner (2005) überträgt dieses Phänomen auf die Forschung zur Bewertung der Stärke kausaler Beziehungen in Abhängigkeit von der Länge des Ursache-Effekt Intervalls. Da hier das Intervall zwischen zwei Versuchsdurchgängen normalerweise nicht mit dem selben Skalierungsfaktor wie das Intervall zwischen Ursache und Effekt verändert wurde kann die beobachtete Abnahme der bewerteten Kausalstärke auf ein methodisches Artefakt zurückzuführen sein. Würden hingegen beide Intervalle so verändert, dass das Verhältnis zwischen ihnen konstant bleibt, so sollte sich die die Bewertung der Kausalstärke nicht verändern.

Nachfolgende Beispiele sollen verdeutlichen, wie stark die Länge des zeitlichen Intervalls zwischen Ursache und Effekt variieren kann und welchen Einfluss Vorwissen über Mechanismen auf die kausale Bewertung dieses Intervalls hat: Verdirbt man sich den Magen durch schlechtes Essen, so können mehrere Stunden zwischen der Ursache (Essen der verdorbenen Speise) und Effekt (Unwohlsein) liegen. Andererseits folgt das Ertönen der Klingel unmittelbar nach dem Betätigen des Klingelknopfes. Das letztgenannte Beispiel verdeutlicht, dass der zeitliche Abstand zwischen Ursache und Effekt so gering sein kann, dass er nicht einmal mehr wahrnehmbar ist. Für die Inferenz einer kausalen Beziehung ist es nun wichtig, dass das zeitliche Intervall nicht zu lang ist um plausibel zu sein. In den oben genannten Beispielen ist die Zeitspanne zwischen Ursache und Effekt sehr unterschiedlich und dennoch sollten in beiden Fällen kausale Beziehungen repräsentiert werden, da die 
zeitlichen Abstände jeweils plausibel sind. Zeitintervalle werden als plausibel angesehen wenn sie mit vorhandenem Wissen in Einklang stehen. Dieses Wissen kann entweder durch Abstraktion vorangegangener Erfahrungen in vergleichbaren Situationen erzeugt oder explizit gelernt werden (vgl. Hagmayer \& Waldmann, 2002). So wissen wir beispielsweise, dass zwischen dem Verzehr von Nahrungsmitteln und dem möglichen Auftreten von Magenschmerzen eine längere Zeitspanne liegt, da es etwas dauert bis unser Körper die Speise soweit umgewandelt (verdaut) hat, dass sich mögliche Schadstoffe negativ auf unser Wohlbefinden auswirken können. Auf der anderen Seite wissen wir auch, dass sich der elektrische Strom, der nach dem Drücken des Klingelknopfes zur Klingel fließt sehr schnell fortbewegt und auch der Mechanismus, der letztendlich zum Ertönen der Klingel führt, sehr schnell abläuft. Zusammen mit Wissen über die Ausbreitungsgeschwindigkeit von Schallwellen sind wir somit wenig überrascht, wenn wir unmittelbar nach dem Drücken des Knopfes das Geräusch der Klingel wahrnehmen. Mit anderen Worten muss der zeitliche Abstand zwischen Ursache und Effekt im Hinblick auf den angenommenen kausalen Mechanismus plausibel sein (vgl. Buehner \& May, 2002, 2003; Buehner, 2005; Greville \& Buehner, 2010; Hagmayer \& Waldmann, 2002). Hagmayer und Waldmann (2002) erbrachten z.B. Belege für die Bedeutung der Dauer von kausalen Wirkmechanismen bei der Schätzung der Kontingenz zwischen Ursachen und Effekten. Versuchspersonen wählten für die Schätzung der Stärke von Kausalzusammenhängen diejenigen Informationen aus, die mit ihrem Vorwissen über die Geschwindigkeit des kausalen Prozesses in Einklang standen.

Ein weiterer Faktor, der beeinflusst wie gut eine Zeitspanne zwischen Ursache und Effekt geeignet ist eine kausale Beziehung $\mathrm{zu}$ repräsentieren, ist die zeitliche Vorhersagbarkeit (temporal predictability) des Effektes durch die Ursache (Greville \& Buehner, 2010). Das Erlernen von kausalen Beziehungen wird erleichtert, wenn der zeitliche Abstand von Ursache und Effekt nicht zu stark variiert also relativ stabil ist. Wie Greville du Buehner (2010) mit aus mehreren Durchgängen bestehenden Lernphasen zeigen konnten führen gleichbleibende zeitliche Abstände zwischen Ursache und Effekt dazu, dass die kausale Beziehung zwischen den beiden Variablen als stärker angenommen wurde als dies bei unregelmäßigen Abständen der Fall war. Der Faktor der zeitlichen Vorhersagbarkeit ist auch mit der Auffassung Humes (1739/1978) vereinbar, für den eine gleichbleibende zeitliche Abfolge von Ursache und Effekt neben der Kovariation eine Voraussetzung für die Wahrnehmung einer Beziehung als kausal darstellt. 
Einige Untersuchungen beschäftigten sich überdies auch mit der räumlichen Nähe von Ursache und Effekt, die bereits von Hume (1739/1978) als Einflussfaktor auf die kausale Inferenz identifiziert wurde. So ist es das Phänomen für des launching effects (Michotte, 1963) entscheidend, dass sich die beiden Objekte berühren, um eine Wahrnehmung von Kausalität beim Beobachter entstehen zu lassen. Daher spielt das Charakteristikum der räumlichen Nähe auch bei den Theorien eine wichtige Rolle, in denen eine kausale Beziehung über einen Prozess der Kraftübertagung konstituiert wird (z.B. das Force-Dynamics Model von Wolff, 2007).

Auch Einhorn und Hogarth (1986, s. Abschnitt 2.4.5.1) betonen neben der Bedeutung der zeitlichen auch die Rolle der räumlichen Kontiguität für die Inferenz kausaler Beziehungen. In ihrem Modell der Bewertung möglicher Ursachen besteht die Rolle von Kontiguität vor allem in der Lenkung von Aufmerksamkeit auf Kontingenzen zwischen Variablen, die dann auf im Hinblick auf ihre kausale Bedeutung betrachtet werden. Ohne Kontiguität würde die Inferenz kausaler Bedingungen somit komplexer und somit schwieriger.

\subsubsection{Gerichtetheit der Beziehung zwischen Ursache und Effekt}

Kausale Beziehungen können in zweifacher Hinsicht als gerichtet beschrieben werden. Zum einen ist die bereits erläuterte zeitliche Abfolge von Ursache und Effekt ein bedeutendes Charakteristikum. Zum anderen ist jedoch auch die (parallel zur zeitlichen Abfolge ablaufende) Wirkrichtung in einer kausalen Beziehung eindeutig determiniert. Ursachen beeinflussen ihre Effekte während Effekte keinen direkten Einfluss auf ihre Ursachen ausüben. In der gebräuchlichen graphischen Darstellung kausaler Beziehungen wird dies durch einen von der Ursache auf den Effekt gerichteten Pfeil zum Ausdruck gebracht (vgl. Abbildung 1). Die Beziehung zwischen der Gerichtetheit einer kausalen Beziehung und der zeitlichen Abfolge von Ursache und Effekt kann wie folgt charakterisiert werden. Die zeitliche Abfolge Ursache $\rightarrow$ Effekt ist zwar notwendig aber nicht hinreichend für die Inferenz von Gerichtetheit. So ist zum Beispiel ein Fall denkbar, bei dem zwei durch eine (versteckte oder offensichtliche) gemeinsame Ursache hervorgerufene Effekte zuverlässig nacheinander auftreten. Hieraus kann nicht auf das Vorliegen einer gerichteten kausalen Beziehung zwischen den beiden Effekten geschlossen werden, da die kausale Beziehung an sich nicht existiert. Im Falle des Vorliegens einer (z.B. durch Intervention festgestellten)kausalen Gerichtetheit in einer Beziehung zwischen zwei Variablen kann hingegen zuverlässig auf die zeitliche Abfolge Ursache $\rightarrow$ Effekt geschlossen werden. 
Kausale Gerichtetheit ist somit hinreichend, aber nicht notwendig für das Vorliegen einer bestimmten zeitlichen Abfolge zwischen zwei Variablen. Als Konsequenz lässt sich aus diesen Überlegungen ableiten, dass es mit Hilfe einer Intervention, die dazu geeignet ist die Richtung der kausalen Wirkung zwischen zwei Variablen festzustellen, möglich ist auch eine Aussage über die zeitliche Abfolge der Variablen zu treffen. Hingegen ist eine exakte Beobachtung der zeitlichen Abfolge zweier Variablen ohne weiteres Wissen über das Vorliegen eines Kausalzusammenhangs nicht ausreichend, um eine Aussage über die Gerichtetheit und damit das Vorliegen einer Kausalrelation treffen zu können. So können beide Variablen von einer dritten (ggf. auch versteckten) Variablen verursacht worden sein ohne dass eine direkte kausale Beziehung zwischen ihnen besteht (vgl. Lagnado et al., 2007). Eine weitere Möglichkeit ist, dass die beobachtete zeitliche Abfolge zufällig entstanden ist, also ohne dass eine dritte, beide beobachtete Variablen verursachende Variable existiert.

Diese Faktoren sprechen gegen das Vermögen von rein auf Informationen über die Kovariation, Kontiguität und zeitlicher Abfolge basierenden Theorien, zuverlässige Aussagen über Kausalrelationen treffen zu können. Weder die assoziativen, noch die logischen Theorien kausaler Inferenz berücksichtigen die Gerichtetheit kausaler Beziehungen. Probabilistische Theorien berücksichtigen hingegen bei der Bewertung von Konvarianzinformationen die Wirkrichtung von Ursache zu Effekt. Bei der damit verbundenen Unterscheidung zwischen Ursache und Effekt handelt es sich jedoch nicht um einen intrinsischen Teil einer kausalen Theorie (Waldmann \& Hagmayer, in Druck). Die PowerPC-Theorie (Cheng, 1997) betrachtet Situationen, in denen die potenziellen kausalen Rollen der betrachteten Variablen bekannt sind und betrachtet daher die Gerichtetheit kausaler Beziehungen nicht. Hingegen geht sie als eine explizite Voraussetzung in die Causal Support Theory (Griffiths \& Tenenbaum, 2005) bei der Beschreibung kausaler Strukturen mit ein. In den kausalen Theorien stellt die Gerichtetheit kausaler Beziehungen einen wesentlichen Bestandteil dar (vgl. Waldmann \& Hagmayer, in Druck).

Waldmann \& Holyoak (1992) liefern empirische Evidenz für die Relevanz der Gerichtetheit kausaler Beziehungen im Bereich des prädiktiven bzw. diagnostischen Schließens. Beim prädiktiven Schließen wird vom Vorliegen einer Ursache-Variablen auf das Vorliegen einer Effekt-Variablen geschlossen, während beim diagnostischen Schließen vom Vorliegen einer Effekt-Variablen auf das Vorliegen einer Ursache-Variablen geschlossen wird. In Bezug auf die Gerichtetheit kausaler Beziehungen bedeutet dies, dass prädiktives Schließen in Richtung der Wirkung des kausalen Zusammenhangs abläuft, während 
diagnostisch entgegen der kausalen Wirkrichtung geschlossen werden muss. Waldmann und Holyoak (1992) zeigten, dass das Auftreten von cue competition davon abhängt, ob die betroffenen Variablen als Ursachen oder Effekte repräsentiert wurden, ob also prädiktive oder diagnostische Schlüsse gezogen werden mussten. Beim Phänomen der cue competition konkurrieren zwei Variablen um die Erklärung eines Effektes. Waldmann und Holyoak bedienten sich bei ihrer Demonstration des blocking-Paradigmas, bei dem zunächst eine Variable A wiederholt mit einem Effekt C gepaart wird, um eine Assoziation zwischen den beiden Variablen zu etablieren. Im Anschluss wird A wiederholt mit einer weiteren Variablen B gepaart, um auch hier eine Assoziation zu etablieren. Danach werden A und B gemeinsam mit C präsentiert. Obwohl A und B jeweils mit C kovariieren schreiben Versuchspersonen nur A eindeutig die Rolle einer Ursache-Variablen zu während sie bezüglich der Rolle von B unsicher sind. Dies wird im Sinne einer cue competition interpretiert: nach der anfänglichen Paarung von A und $\mathrm{C}$ ist es eindeutig, dass A eine Ursache von C ist. Wird nun zusätzlich zu A noch B präsentiert und C tritt wiederrum auf, so liefert B keine weiteren Informationen bei der Vorhersage von $\mathrm{C}$ und wird daher im Vergleich zu A weniger stark als Ursache von C wahrgenommen. Dies lässt sich noch problemlos mit auf reinen Kovarianzinformationen beruhenden assoziativen Theorien erklären. Wie Waldmann und Holyoak aber zeigen konnten tritt der Blocking-Effekt nur in der prädiktiven Bedingung auf, d.h. wenn die Variablen A und B Ursachen von C darstellen. In der diagnostischen Bedingung, bei der C als Ursache von A und B instruiert wird verschwindet der Effekt bei sonst gleichem Versuchsablauf. Dieses Phänomen ist ein Beleg für die Bedeutung der Gerichtetheit von Kausalrelationen (von der Ursache zum Effekt). Diese Eigenschaft wird in reinen Kovarianzinformationen nicht repräsentiert und spielt in den assoziativen Theorien folglich keine Rolle.

\subsubsection{Rolle von Interventionen}

Wie bereits im Abschnitt über die Gerichtetheit von Kausalbeziehungen erwähnt, sind Interventionen in kausale Systeme eine Möglichkeit, etwas über die Art der sie konstituierenden Kausalrelationen in Erfahrung zu bringen. Es handelt sich somit also um ein Instrument mit dem Kausalrelationen identifiziert und differenziert werden können (vgl. Lagnado et al., 2007). Darüber hinaus besteht eine wichtige Aufgabe im Bereich des kausalen Urteilens und Entscheidens in der Vorhersage der Folgen möglicher Handlungen, die mittels mentaler Simulation von Interventionen in Kausalmodelle getroffen werden können. Dies ist der Gegenstand der Causal Model Theory of Choice (Sloman \& Hagmayer, 2006; Hagmayer 
\& Sloman, 2009), die an späterer Stelle noch ausführlicher behandelt wird. Durch die Auswahl der für ein gegebenes Kausalmodell besten Intervention besteht zudem die Möglichkeit mit Hilfe von Interventionen vorhandenes Wissen über Kausalsysteme zu testen.

Eine Intervention (vgl. Hagmayer, Sloman, Lagnado \& Waldmann, 2007) setzt eine Variable in einem kausalen Modell zuverlässig auf einen bestimmten Wert. Dies geschieht unabhängig von den normalen Ursachen der Variablen auf die interveniert wird, diese verlieren somit ihren Einfluss. Die kausale Verbindung zwischen der Variablen, auf die interveniert wird und ihren Effekten bleibt hingegen bestehen. Daher kann mit Hilfe einer Intervention die Wirkung einer Variablen auf ihre Effekte isoliert beobachtet werden. Voraussetzung hierfür ist, dass die Intervention selbst die Effekt-Variable nicht beeinflusst und statistisch unabhängig von allen anderen Faktoren ist, die einen direkten oder indirekten Einfluss auf die Effekt-Variable ausüben können (Waldmann \& Hagmayer, 2005). In der formalen Darstellung kausaler Systeme (Causal Bayes Nets, Glymour, 2001; Pearl, 2009) werden Interventionen aufgrund ihrer Fähigkeit des Isolierens bestimmter Teile eines Systems auch bildhaft als graph surgery (Pearl, 2009) bezeichnet.

Erfüllt eine Intervention die genannten Bedingungen, kann die Gerichtetheit kausaler Beziehungen wie folgt festgestellt werden: Interveniert man auf eine Ursache-Variable, so hat dies (ggf. probabilisitische) Auswirkungen auf die Ausprägungen der mit ihr verbundene(n) Effekt-Variable(n). Interveniert man hingegen auf eine Effekt-Variable, so wird eine mit ihr verbundene Ursache-Variable hiervon nicht beeinflusst. Aus (wiederholten) Beobachtungen der Auswirkungen von Interventionen kann sowohl auf strukturelle Merkmale (die Gerichtetheit der Beziehung zwischen den beteiligten Variablen) als auch auf die Stärke der Beziehung geschlossen werden.

Empirische Evidenz für den Nutzten von Interventionen bei der Inferenz kausaler Strukturen liefern u.a. die Studien von Steyvers, Tenenbaum, Wagenmakers und Blum (2003) sowie Lagnado und Sloman (2004). Versuchspersonen hatten hier die Aufgabe kausale Strukturen entweder mit Hilfe von Beobachtungen oder mit Interventionen zu identifizieren. Es zeigten sich bessere Ergebnisse wenn die Versuchspersonen Interventionen durchführen durften, auch wenn die Leistungen nicht optimal waren. Weitere Studien (Meder, Hagmayer \& Waldmann, 2008; Sloman \& Lagnado, 2005; Waldmann \& Hagmayer, 2005) konnten außerdem zeigen, dass Menschen in der Lage sind, Vorhersagen für die Folgen von Interventionen abzuleiten, die zuvor noch nie durchgeführt oder beobachtet wurden. 
Da die Rolle von Interventionen auf der Grundlage des nur in kausalen Theorien existierenden Charakteristikums der Gerichtetheit kausaler Beziehungen basiert, wird sie auch nur von einigen dieser Theorien betrachtet. Da kovariationsbasierte Theorien nicht davon ausgehen, dass Menschen die Richtung von Kausalrelationen repräsentieren, haben Interventionen für sie folglich auch keine Bedeutung.

\subsubsection{Mechanismen zwischen Ursachen und Effekten}

Ein bekanntes Beispiel für Bedeutung von Mechanismen bei Kausalattributionen stammt von Sober (1987 zit. n. Sober, 2001): Angenommen es gäbe eine über lange Zeit stabile, starke Korrelation zwischen den Preisen für Brot in England und der Höhe des Meeresspiegels in Venedig. Dennoch wäre es höchst ungewöhnlich, eine gemeinsame Ursache für die Höhe des Brotpreises und das Niveau des Meeresspiegels anzunehmen, da kein plausibler Mechanismus verfügbar ist, der die beiden Ereignisse kausal miteinander verbindet.

Glennan (2009) bezeichnet die Entdeckung von Mechanismen als „Goldstandard“ (gold standard) bei der Erklärung kausaler Beziehungen und beschreibt Ansätze für eine mechanistische Analyse von Kausalität. In der Literatur existieren daher eine Vielzahl von Beschreibungen, die versuchen das Wesen dieser kausalen Mechanismen zu erfassen. Einige Ansätze orientieren sich beispielsweise an den Prozessen physikalischer Kraftübertragung, bei die einem Objekt innewohnende Kraft durch physischen Kontakt mit einem anderen Objekt ( zumindest teilweise) auf dieses übergeht (z.B. der conserved quantity-Ansatz von Dowe, 1992). Exemplarisch können hier die Vorgänge beim Billardspiel erwähnt werden, bei dem zunächst eine bestimmte Kraft durch den Stoß auf die weiße Kugel übertragen wird. Trifft die weiße anschließend auf eine andere Kugel, so wird ihre Kraft wiederrum auf die andere Kugel übertragen. Einen auf diesen und ähnlichen Beobachtungen und der damit verbundenen intuitiven Zuschreibung von Kausalität basierenden psychologischen Ansatz lieferte Michotte (1963) mit seinen Untersuchungen zur wahrgenommenen Kausalität, auf den an späterer Stelle noch näher eingegangen wird.

Einen alternativen Standpunkt vertritt Glennan (2009), der Mechanismen als komplexe Systeme versteht, die über Interaktionen ihrer Teile Verhalten erzeugen. Dabei müssen die interagierenden Teile in einer direkten kausalen Beziehung zueinander stehen. Ein verwandter philosophischer Ansatz, der Mechanismen ebenfalls als über ihre Teile interagierende Systeme versteht, sieht Mechanismen hingegen als aus Entitäten und Aktivitäten 
zusammengesetzt (Machamer, Darden \& Craver, 2000). Dieser Ansatz geht davon aus, dass Aktivitäten Veränderungen produzieren und diese Produktion Kausalität konstituiert (Machamer, 2004). Den System-orientierten Ansätzen ist gemein, dass sie in der Lage sind, ein großes Spektrum von Phänomenen abzudecken, während Zweifel an der Generalisierbarkeit der an physikalischen Prozessen orientierten Ansätze über die Domäne physikalischer Phänomene hinaus bestehen (vgl. Waldmann \& Hagmayer, in Druck).

Den im Rahmen dieser Arbeit betrachteten psychologisch-kausalen Theorien zufolge werden die Verbindungen zwischen Ursachen und ihren Effekten über kausale Mechanismen hergestellt, die eine Wirkung der Ursache auf ihren Effekt ermöglichen. Im Rahmen der Causal Model Theory (Waldmann, 1996; Waldmann \& Holyoak, 1992; Waldmann, Holyoak \& Fratienne, 1995) beispielsweise bildet die Struktur des kausalen Modells gerichtete Mechanismen $a b$, die Ursachen mit ihren Effekten verbinden. Darüber hinaus werden in dieser Theorie Mechanismus-basierte Informationen mit Kovariations-basierten Informationen kombiniert, da Letztere dazu genutzt werden können, die Parameter des kausalen Modells zu inferieren (vgl. Waldmann \& Hagmayer, in Druck).

Wie bereits aus dem eingangs dieses Abschnittes erwähnten Beispiel hervorgeht, spielt die Plausibilität kausaler Mechanismen eine wichtige Rolle. Plausible Mechanismen werden leichter repräsentiert als solche, die nicht plausibel erscheinen (vgl. Ahn \& Kalish, 2000; Fugelsang \& Thompson, 2003). Ebenso werden Ereignisse, die in einem Kausalmodell nicht über Mechanismen miteinander verbunden sind eher als zufällig interpretiert bzw. beim kausalen Schließen weniger stark gewichtet (Ahn \& Kalish, 2000). Waldmann \& Hagmayer (2001) konnten zeigen, dass die Abstraktheit der präsentierten Informationen über ein Kausalsystem einen Einfluss auf die Plausibilität und damit auf vorgenommene Kausalurteile ausübt. Kausalrelationen werden stärker eingeschätzt, wenn sie in einem für die Versuchspersonen plausiblen bzw. konkreten Kontext stehen als dies bei einem sehr abstrakten Kontext der Fall ist (Experiment 3). Die Autoren führen diesen Befund darauf zurück, dass es den Versuchspersonen mit einer plausiblen Coverstory leichter fiel den beteiligten Variablen ihre Rollen im Kausalsystem zuzuweisen, da sie hierbei auf bereits vorhandenes Wissen über vergleichbare kausale Mechanismen zurückgreifen konnten. Dies war im Rahmen einer abstrakten Coverstory nicht der Fall.

Im Folgenden werden Theorien behandelt, die untersuchen welchen Einfluss Wissen über Mechanismen im Bereich des kausalen Denkens ausübt. Im Vordergrund steht dabei die Betrachtung der psychologischen Prozesse des kausalen Schließens während die 
metaphysische Perspektive nur kurz angerissen wird. Als ein wesentliches Problem dieser mechanistischen Theorien identifiziert Glennan (2009; vgl. auch Ahn, Kalish, Medin \& Gelman, 1995; Goldvarg \& Johnson-Laird, 2001) die Frage nach der Grundlage kausaler Mechanismen. Versucht man einen Mechanismus in einzelne Komponenten aufzuteilen und diese wiederrum über Mechanismen zu erklären, so führt dies entweder zu einer unendlichen Kette von Mechanismen (einem infiniten Regress) oder aber der Feststellung, dass Mechanismen in letzter Konsequenz aus anderen Komponenten bestehen müssen. Damit ist der grundsätzliche Charakter von Mechanismen für die Erklärung von Kausalität in Frage gestellt. Alternative Erklärungsansätze weisen allerdings ähnliche Probleme auf. Manipulationsbasierte Ansätze, die versuchen Kausalrelationen mit Hilfe von Interventionen auf die beteiligten Variablen zu identifizieren, benötigen auf einer fundamentalen kausalen Ebene beispielsweise Annahmen über einen der Interventionswirkung zu Grunde liegenden Mechanismus, um gesicherte Aussagen über eine Kausalrelation machen zu können. Ohne diese Annahmen wären lediglich Vermutungen über die Rolle von Ursache und Effekt möglich, da auf der fundamentalen Ebene auf der Basis von Beobachtungen nicht sicher bestimmt werden kann, welche Variable welche Rolle einnimmt (Glennan, 2009).

Neben den philosophischen Ansätzen existiert auch eine Reihe psychologischer Theorien, die kausale Mechanismen als einen wesentlichen Bestandteil kausalen Schließens betrachten. Eine davon ist die Theory of Causal Processing (White, 1989), die von der Existenz eines allgemeinen Konzeptes von Kausalität ausgeht, in dem generative Mechanismen die Erzeugung von Effekten durch ihre Ursachen ermöglichen. Der Vorgang, durch den eine Ursache einen Effekt erzeugt wird, hier als die Freisetzung einer kausalen Kraft (causal power) interpretiert, die unter bestimmten Bedingungen (releasing conditions) erfolgt. Die kausale Stärke wird dabei als stabile Eigenschaft von Dingen (wie physischen Objekten oder Personen) verstanden. Die kausale Stärke wirkt jedoch nicht direkt auf den Effekt, sondern vermittelt durch eine Übertragung (transmission) einer Kraft oder Energie von der Ursache auf den Effekt (White, 2009). In einer konkreten Situation wird dieses basale Kausalitäts-Konzept mit Kontext-spezifischen Annahmen gefüllt.

White (1989) legt nahe, dass die generativen Beziehungen zwischen Ursache und Effekt über einen Entwicklungsprozess in der Kindheit gelernt werden. Zunächst würden Kinder kausale Verarbeitung auf der Basis eines Eigenschaftstransfers zwischen zwei Objekten durchführen. Dieser Transfer basiert wiederrum auf der Wahrnehmung von kontinuierlichen Bewegungscharakteristika, die sich über zwei als getrennt wahrgenommene 
Objekte ausdehnen. Die Kontinuität hat ihrerseits verschiedene invariante Eigenschaften: zeitliche Kontiguität, räumliche Kontiguität, zeitlicher Vorrang der ,Ursache“ vor dem ,Effekt‘ und Ähnlichkeit der kausal relevanten Eigenschaften vor und nach dem Transfer.

Die psychologischen Prozesse kausalen Schließens stehen im Mittelpunkt des Mechanismus-basierten Ansatzes von Ahn und Kollegen (1995). Sie gehen davon aus, dass Wissen oder Vermutungen über Mechanismen grundlegende Komponenten kausalen Schließens darstellen. Mechanismen stellen eine Verbindung zwischen mehreren an einem Ereignis beteiligten „Oberfächen-Faktoren“ (,surface“ factors) her und werden als Komponenten des Ereignisses verstanden, die kausale Kräfte oder kausale Notwendigkeit besitzen (,... some component of an event which is thought to have causal force or causal necessity“, Ahn et al., 1995, S. 303). Wissen über Mechanismen geht nach Ahn und Kollegen somit über direkt Beobachtbares hinaus, es besteht im Verständnis des Prozesses über den eine Ursache ihren Effekt hervorruft. Es besitzt ein anderes Abstraktionsniveau als das konkret vorliegende Ereignis und beinhaltet Entitäten oder Prozesse, die nicht konkret beschrieben wurden. Daher werden Mechanismen auch als theoretische Entitäten verstanden, die theoretische Konstrukte beinhalten, welche dazu dienen, vorliegende Beobachtungen kausal interpretieren zu können.

Ein ungelöstes Problem ist die Frage nach der Herkunft des Wissens über Mechanismen. Ahn et al. (1995; Ahn \& Kalish, 2000) legen nahe, dass dieses Wissen aus verschiedenen Quellen stammen kann. Es kann angeboren, über Kommunikation erworben oder durch Analogiebildung entstanden sein. Allerdings muss z.B. das für die Analogiebildung benutzte Wissen seinerseits eine Herkunft aufweisen, so dass sich schnell ein infiniter Regress ergibt. Nach Auffassung von Ahn und Kalish (2000) ist dieser infinite Regress jedoch mehr ein wissenschaftstheoretisches oder philosophisches Problem, das im typischerweise stattfindenden kausalen Schließen kaum eine Rolle spielt und daher für ihren Ansatz von nachrangiger Bedeutung ist. Vertreter kovatiationsbasierter Ansätze gehen davon aus, dass das Wissen über kausale Mechanismen daher letztendlich auf Analysen beobachteter Kovariationen basieren muss. So versteht Glymour (1998) einen Mechanismus als diejenige Komponente, die eine bestehende Korrelation zwischen zwei Variablen gegeben den Mechanismus verschwinden lässt (d.h. $M$ ist ein Mechanismus, wenn $\operatorname{Korr}(A, B \mid M)=0$ und $\operatorname{Korr}(\mathrm{A}, \mathrm{B}) \neq 0$ ). Glymour vertritt den Standpunkt, dass Mechanismen Muster aus Kovariationen sind und nicht nur Evidenz für diese darstellen. Somit ist eine Trennung von 
Mechanismen und Kovariationsmustern wie sie beispielsweise Ahn und Kollegen (1995) vornehmen nach Ansicht Glymours nicht sinnvoll.

Wie Ahn und Kalish (2000) jedoch zeigen ist auch diese Sichtweise mit Problemen behaftet. So kann aus der obigen Definition abgeleitet werden, dass alle Kovariationsmuster, bei denen eine Variable eine Korrelation zwischen zwei anderen Variablen verschwinden lassen kann einen Mechanismus beschreibt. Bestehen jedoch logische Abhängigkeiten zwischen den am Ereignis beteiligten Variablen (also dem potenzellen Mechanismus und den beiden korrelierten Variablen), so würde der kovariationsbasierte Ansatz Glymours (1998) von einem Mechanismus ausgehen, der aufgrund der Abhängigkeit tatsächlich nicht existiert. Ahn und Kalish (2000) stellen daher fest, dass bedingte Kovariation nicht hinreichend für die Definition von Mechanismen ist.

Weitere Probleme des kovariationsbasierten Ansatzes identifizieren Ahn und Kalish (2000; Ahn et al., 1995) in der Notwendigkeit mehrerer Beobachtungen (mindestens die Ausprägung der Effektvariable bei An- sowie Abwesenheit einer potentiellen Ursache), die diese Ansätze erfordern. Diese macht es beispielswiese nicht möglich, eine Kausalattribution basierend auf nur einer einzelnen spezifischen Beobachtung vorzunehmen. Die rechnerische Komplexität von rein auf Informationen über Kovariation beruhenden Analysen spricht ebenfalls gegen diesen Ansatz. Ohne Vorwissen wäre es nicht möglich, potentielle Ursachen vorzuselektieren. Daher müssten Kovariationen zwischen allen verfügbaren Variablen untersucht werden. Dies läge jedoch weit außerhalb der Kapazität des kognitiven Apparates, da bereits zwischen relativ wenigen Variablen eine große Anzahl an Kovariationen (n*(n1)/2) besteht. In der Realität verfügen Menschen außerdem, (so gut wie) nie über repräsentative Stichproben auf deren Basis sie kovariationsbasierte Schlüsse ziehen könnten. Des Weiteren ist es mitunter praktisch unmöglich, sämtliche benötigte Evidenz überhaupt zu erhalten (wenn es z.B. darum geht, bestimmte Faktoren konstant zu halten). Diese Probleme des kovariationsbasierten Ansatzes veranlassen Ahn und Kalish (2000) zu dem Schluss, dass Menschen im typischen Fall kausale Attributionen nicht auf der Grundlage von Kovariationen, sondern über die Prüfung der Plausibilität von Mechanismen bilden. Dies bedeutet jedoch nicht, dass Informationen über Kovariationen keine Rolle bei der Identifikation kausaler Relationen spielen. In vielen Situationen wie beispielsweise der Bestimmung potentieller Ursachen eines Effektes bestehen vielmehr komplexe Beziehungen zwischen Mechanismen und Kovariationen. Ahn und Kalish fassen ihre Ansicht über die Beziehung zwischen Assoziationen und Mechanismen wie folgt zusammen: „Thus, we argue 
that association is related to mechanism as evidence ist to theory. That is, mechanism explains association as theory explains evidence." (Ahn \&Kalish, 2000, S. 204.)

\subsubsection{Zusammenfassung}

Der vorangegangene Abschnitt beschäftigte sich mit den verschiedenen Charakteristika kausaler Beziehungen und ihrer Berücksichtigung in Theorien kausalen Denkens. Einige dieser Theorien verstehen kausales Denken als Spezialfall einer allgemeineren-domänenübergreifenden Denkfähigkeit während andere eine Eigenständigkeit kausalen Denkens im kognitiven Apparat annehmen. Einige der vorgestellten Charakteristika werden in fast allen vorgestellten Theorien berücksichtigt. Dazu gehören das gemeinsame Auftreten von Ursache und Effekt, die zeitliche Priorität der Ursache vor dem Effekt, sowie die zeitliche und/oder räumliche Kontiguität zwischen Ursache und Effekt. Dagegen werden die Charakteristika Gerichtetheit der kausalen Beziehung und die damit verbundene Rolle von Interventionen, sowie die Annahme von Mechanismen bei der Bildung kausaler Beziehungen von den kovariationsbasierten und den logischen Theorien nicht berücksichtigt. Es konnte jedoch anhand der Ergebnisse verschiedener empirischer Studien gezeigt werden, dass auch diese Charakteristika kausaler Beziehungen einen Einfluss auf menschliches Verhalten ausüben und daher in Theorien kausalen Denkens berücksichtigt werden sollten. Da dies nur in den kausalen Theorien der Fall ist kann somit davon ausgegangen werden, dass kausales Denken eine eigenständige Form des Denkens darstellt.

\subsection{Prozesse kausalen Denkens in Zwei-Prozess-Theorien der Urteils- und}

\section{Entscheidungsforschung}

Nachdem im vorangegangenen Abschnitt dargelegt wurde, dass Menschen die verschiedenen Charakteristika kausaler Beziehungen in ihrem Denken berücksichtigen, soll im Folgenden untersucht werden, welche Prozesse die Grundlage des kausalen Denkens bilden könnten. Dazu wird zunächst die in der Urteils- und Entscheidungsforschung vielfach Verwendung findende Klasse der Zwei-Prozess-Theorien (vgl. Evans \& Frankish, 2009; Stanovich, 2011 für Überblicksarbeiten; für Kritik an den Zwei-Prozess-Theorien vgl. Osman, 2004; Keren \& Schul, 2009) vorgestellt. Vertreter dieser Ansätze gehen davon aus, dass es zwei unterschiedliche Arten von kognitiven Prozessen mit unterschiedlichen Merkmalen gibt. Es wird untersucht, wie die verschiedenen Theorien verschiedene Aspekte kausalen Denkens berücksichtigen und welche Art von Prozessen hierbei ggf. angenommen wird. Im Anschluss soll untersucht werden, wie sich verschiedene Theorien kausalen Denkens in Bezug auf die 
zwei Arten von Prozessen positionieren bzw. positioniert werden können. Abschließend wird die Notwendigkeit einer einheitlichen Untersuchung kausaler Theorien vor dem Hintergrund der Zwei-Prozess-Theorien diskutiert und Überlegungen zum im empirischen Teil dieser Arbeit verwendeten Untersuchungsparadigma angestellt.

Zwei-Prozess- bzw. Zwei-System-Theorien beschäftigen sich mit den kognitiven Vorgängen, die dem menschlichen Denken, Urteilen und Entscheiden zu Grunde liegen. Die Gemeinsamkeit zwischen diesen Theorien besteht darin, dass sie von der Annahme der Existenz zweier unterschiedlicher Arten von kognitiven Prozessen bzw. Systemen ausgehen, die unterschiedliche Merkmale aufweisen. Die Begriffe Prozess und System werden in den verschiedenen Theorien uneinheitlich verwendet. Im Rahmen der vorliegenden Arbeit wird der Begriff Prozess in Anlehnung an Frankish und Evans (2009) für grundlegende kognitive Verarbeitungsmechanismen verwendet, während Systeme als Bestandteile der mentalen Architektur verstanden werden, die aus verschiedenen Prozessen bestehen können und auf Grundlage der Prozesseigenschaften operieren. Dabei ist es wichtig klarzustellen, dass der hier verwendete Systembegriff keine Aussage über hirnphysiologische Korrelate implizieren soll (zu vergleichbaren Ansichten s. z.B. Evans, 2008; Stanovich, 2004). Obwohl der Fokus dieses Abschnittes auf den kognitiven Prozessen kausalen Denkens liegt, ist es nicht möglich, auf den System-Begriff zu verzichten, da dieser im Fokus von mehreren hier vorgestellten Ansätzen steht. Manche dieser Ansätze beschreiben zwar darüber hinaus auch die den Systemen zu Grunde liegenden Prozesse, dies ist aber nicht immer der Fall.

\subsubsection{Ursprünge der modernen 2-Prozess-Theorien}

In den letzten Jahrzehnten hat sich eine Vielzahl von Theorien entwickelt, die sich mit der Unterscheidung zweier verschiedener Arten von kognitiven Prozessen beschäftigen (für einen Überblick vgl. Evans, 2008; Evans \& Frankish, 2009; Stanovich, 2011). Wie Frankish und Evans (2009) in einer kurzen Vorstellung der Entwicklung von Zwei-Prozess-Theorien anmerken, entstanden diese Theorien in verschiedenen Gebieten der Psychologie weitgehend unabhängig voneinander. Die Ursprünge der modernen Zwei-Prozess-Theorien liegen in den siebziger Jahren des 20. Jahrhunderts.

Eine der ersten modernen Zwei-Prozess-Theorien ist im Bereich des schlussfolgernden Denkens angesiedelt und stammt von Wason und Evans (1975). Die Autoren untersuchten Handlungsbegründungen von Versuchspersonen bei der Wason selection task (Wason, 1966, 1968a zit. n. Wason \& Evans, 1975). Bei dieser Aufgabe werden Versuchspersonen 
aufgefordert, konditionale Aussagen der Form „Wenn P, dann Q“ (Affirmation) und „Wenn $\mathrm{P}$, dann nicht-Q“ (Negation) zu überprüfen, wobei $\mathrm{P}$ in der Regel ein bestimmter Buchstabe und Q eine Zahl repräsentiert. Den Versuchspersonen werden vier Karten zusammen mit der Information präsentiert, dass jede der Karten auf der einen Seite einen Buchstaben und auf der anderen Seite eine Zahl aufweist. Ihre Aufgabe besteht dann darin, konditionale Aussagen der Form „Wenn auf der einen Seite ein Vokal (P), dann auf der anderen Seite eine gerade Zahl (Q)“ durch das Herumdrehen von möglichst wenigen Karten zu prüfen. In verschiedenen Experimenten konnte gezeigt werden, dass Versuchspersonen systematisch Fehler beim Herumdrehen der Karten begehen. So wurden sowohl bei Affirmations- (Wenn P dann Q) als auch bei Negations-Aufgaben (Wenn P dann nicht Q) vorzugsweise die gleichen Karten (nämlich P und Q) umgedreht, obwohl dieses Verhalten nur bei Negationsaufgaben korrekt ist (bei Affirmation, wäre das Herumdrehen von P und nicht-Q korrekt). Wason und Evans (1975) untersuchten daraufhin schriftlich gegebene Begründungen von Versuchspersonen für ihre Wahl der herumzudrehenden Karten nach systematischen Tendenzen. Die Ergebnisse des durchgeführten Experimentes werden von den Autoren als Beleg für das Wirken zweier unterschiedlicher Prozesse während der Bearbeitung der eigentlichen Aufgabe (dem Herumdrehen der Karten) und der nachfolgenden Introspektion (der Begründung der Wahl) gewertet. Dabei werden zwei fundamentale Annahmen gemacht: 1. Die bei der Bearbeitung der Aufgabe ablaufenden Prozesse sind der Introspektion nicht ohne Weiteres zugänglich. 2. Die Introspektion bezieht sich nicht auf die eigentliche Aufgabe, sondern besteht in der Konstruktion einer Erklärung des eigenen Verhaltens in Übereinstimmung mit vorhandenem Wissen über die Entscheidungssituation.

Auch wenn die von Wason und Evans (1975) postulierten Prozesse sehr spezieller Natur sind, so sind doch bereits wesentliche Merkmale allgemeinerer Zwei-Prozess Theorien erkennbar: Die beiden Arten von Prozessen unterscheiden sich in der Notwendigkeit des Mitwirkens von Bewusstsein. Auf der einen Seite gibt es schnelle Verarbeitungsprozesse, die unbewusst ablaufen und auch nicht ohne Weiteres bewusst gemacht werden können. Andererseits gibt es bewusste Prozesse, die beispielsweise dann ablaufen, wenn eine Begründung für ein zuvor gezeigtes Verhalten konstruiert werden soll.

Eine weitere frühe Zwei-Prozess-Theorie stammt von Schneider und Shiffrin (1977; Shiffrin \& Schneider, 1977). Sie postulieren Unterschiede zwischen automatischen und kontrollierten Prozessen der Informationsverarbeitung. Dabei wird automatische Verarbeitung als die Aktivierung einer gelernten Sequenz im Langzeitgedächtnis durch geeignete Stimuli 
verstanden, die dann ohne Kontrolle durch das Individuum abläuft und nicht an Aufmerksamkeit oder Kapazitätsbegrenzungen gebunden ist. Kontrollierte Verarbeitung wird nach Schneider und Shiffrin über wiederholte und konsistente Verknüpfung von Variablen (z.B. Stimulus und Reaktion) erzeugt, wobei dieser Prozess zunächst kontrolliert abläuft und erst mit entsprechender Anzahl an Wiederholungen automatisiert wird.

Im Gegensatz dazu wird die kontrollierte Verarbeitung als eine Aufmerksamkeit erfordernde, willentlich steuerbare, zeitweise Aktivierung einer Sequenz von Elementen verstanden, die zwar schnell und einfach auch in unbekannten Situationen erreicht werden kann, aber den Kapazitätsbegrenzungen des kognitiven Systems unterliegt. Darüber hinaus ist die kontrollierte Verarbeitung meistens von serieller Natur. Sie ermöglicht die Speicherung von Inhalten in das Langzeitgedächtnis. Aufmerksamkeit ist neben den Unterschieden in der Kapazität ein wichtiger Faktor bei der Diskriminierung der beiden Arten von Prozessen, da sie bei kontrollierten Prozessen zwingend, bei automatischen Prozessen hingegen nicht notwendig anwesend sein muss.

Anhand der beiden vorgestellten frühen Zwei-Prozess-Theorien wird bereits deutlich, dass Aufmerksamkeit bzw. Bewusstsein eine wichtige Rolle bei der Unterscheidung zwischen den jeweils postulierten Prozessen spielt. Die sich in der Folge weitgehend unabhängig voneinander entwickelnden Zwei-Prozess-Theorien auf verschiedenen Gebieten der Psychologie (vgl. Frankish \& Evans, 2009) nehmen diese Unterscheidung ebenfalls vor. Darüber hinaus weisen diese Ansätze eine Vielzahl von weiteren Gemeinsamkeiten auf (vgl. Evans, 2008 für einen Überblick).

\subsubsection{Gemeinsamkeiten verschiedener Zwei-Prozess-Theorien}

Inzwischen existieren mehrere Arbeiten, die Prozess-Charakteristika verschiedener Ansätze zusammengetragen und analysiert haben (z.B. Evans, 2003, 2008; Stanovich, 1999; Stanovich \& Toplak, 2012). So unterteilt beispielsweise Evans (2008) die in der Literatur vorkommenden Eigenschaften in vier Gruppen. Dabei werden die einzelnen Eigenschaften in Abhängigkeit ihrer Ausprägung entweder dem schnellen, automatischen, unbewussten und mit hoher Kapazität ausgestatteten System 1 oder dem langsamen, bewussten und deliberierenden System 2 zugeordnet. Die erste Gruppe von Eigenschaften wird von Evans (2008, Tabelle 2) unter der Überschrift Bewusstheit (consciousness) aufgelistet und umfasst die Begriffspaare unbewusst/bewusst, implizit/explizit, automatisch/kontrolliert, hoher Aufwand/niedriger Aufwand, schnell/langsam, hohe Kapazität/niedrige Kapazität, 
standardmäßige Prozesse/inhibitorische Prozesse, holistisch-wahrnehmungsbasiert/analytischreflektiv. Dabei bezieht sich der jeweils erste Begriff eines Paares auf die mit System 1 und der zweite Begriff eines Paares auf die mit System 2 assoziierte Eigenschaftsausprägung. In der zweiten Gruppe werden diejenigen Eigenschaften zusammengefasst, die mit der Entwicklung (evolution) der beiden Systeme in Verbindung gebracht werden können. Die aufgeführten Begriffspaare sind hier evolutionär alt/evolutionär neu, evolutionäre Rationalität/ individuelle Rationalität, geteilt mit anderen Tieren/einzigartig menschlich, nonverbal/verbunden mit Sprache, modulare Kognition/fluide Intelligenz. Die dritte Gruppe besteht aus funktionalen Merkmalen (functional characteristics) der beiden Systeme. Sie wird durch die Begriffspaare assoziativ/regelbasiert, domänenspezifisch/domänenübergreifend, kontextabhängig/Abstrakt, pragmatisch/logisch, parallel/sequenziell, sowie stereotypisch/egalitär gebildet. Die letzte Gruppe beschäftigt sich mit der Beziehung individueller Unterschiede (individual differences) zu den beiden Systemen. Sie besteht aus den Eigenschaftsausprägungen universal/vererbbar, unabhängig von allgemeiner Intelligenz/verbunden mit allgemeiner Intelligenz, unabhängig vom Arbeitsgedächtnis/begrenzt durch das Arbeitsgedächtnis.

Die Vielzahl der entstandenen Theorien und die aufälligen Gemeinsamkeiten der von ihnen angenommenen Systeme veranlassten Stanovich (1999) das auch von Evans (2008) verwendete Begriffspaar System 1/ System 2 in die Debatte einzuführen. Obwohl es in der Literatur vielfach Anwendung gefunden hat, plädiert Evans $(2009,2011)$ inzwischen für eine Unterscheidung zwischen Typ 1 und Typ 2 Prozessen als Komponenten von Typ 1- und Typ 2-Systemen. Der Grund hierfür liegt in möglichen falschen Interpretationen der Begriffe System 1 und System 2. Diese legen nach Evans (2011) die unrealistische Annahme nahe, dass es genau zwei distinkte Verarbeitungssysteme gibt, die in Lage sind, verschiedenste Arten von Aufgaben zu bewältigen. Darüber hinaus kann schnell der falsche Eindruck entstehen, die beiden Verarbeitungssysteme entsprechen zwei distinkten Systemen im Gehirn (Stanovich \& Toplak, 2012).

Evans (2011) vertritt hingegen die Ansicht, dass verschiedene Arten von Aufgaben (z.B. Planen von Handlungen, Lesen oder das Testen von Hypothesen) von unterschiedlichen Systemen des gleichen Typs (hier: Typ 2) bearbeitet werden. Dabei können an der Bewältigung einer Aufgabe (z.B. ein komplexes Problem im Bereich des deduktiven Schließens) auch mehrere Typ 2-Systeme beteiligt sein, die auf unterschiedliche Ressourcen zugreifen. Typ 2-Systeme beinhalten immer Typ 2-Verarbeitungsprozesse und teilen dadurch 
deren gemeinsames Merkmal des Zugriffes auf ein singuläres, zentrales Arbeitsgedächtnissystem. Diese Notwendigkeit der Beteiligung des Arbeitsgedächtnisses an Typ 2-Systemen kann Evans zufolge auch als Notwendigkeit kontrollierter Aufmerksamkeit verstanden werden. Neben den Typ 2-Verarbeitungsprozessen beinhalten Typ 2-Systeme überdies auch Typ 1-Prozesse, die außerhalb des Arbeitsgedächtnisses operieren. Als ein Beispiel nennt Evans den Vorgang des Lesens, der auf der einen Seite Aufmerksamkeit erfordert und damit ein Typ-2 System konstituiert. Auf der anderen Seite sind aber am Lesen auch Typ 1-Prozesse (z.B. während der Informationsaufnahme und des Sprachverständnisses) beteiligt. Typ 2-Systeme bestehen somit aus einer Kombination von Typ 1- und Typ 2Verarbeitungsprozessen. Damit lassen sie sich auch gegen Typ 1-Systeme abgrenzen, die keinen Zugriff auf das Arbeitsgedächtnis benötigen, da sie ausschließlich aus Typ 1Verarbeitungsprozessen bestehen. Inzwischen wird das Begriffspaar Typ 1- / Typ 2-Prozess in der Literatur zu Zwei-Prozess-Theorien zunehmend genutzt (vgl. Evans, 2009, 2011; Samuels, 2009; Stanovich, 2011; Stanovich \& Toplak, 2012). Daher soll es auch im weiteren Verlauf dieser Arbeit Anwendung finden.

Neben den Anforderungen an das Arbeitsgedächtnis gibt es noch weitere charakteristische Merkmale von Typ 1- bzw. Typ 2-Prozessen, die den zuvor beschriebenen Eigenschaften von System 1 bzw. System 2 entsprechen. Evans $(2009,2011)$ charakterisiert Typ 1-Prozesse zusammenfassend als schnell, unabhängig von Arbeitsgedächtnis und kognitiven Fähigkeiten, mit hoher Kapazität ausgestattet und automatisch ablaufend. Als zentrale Eigenschaft betrachtet er dabei die Unabhängigkeit vom Arbeitsgedächtnis (Evans, 2008, 2011). Dagegen laufen Typ 2-Prozesse langsam und kontrolliert ab und sind abhängig von individuellen kognitiven Fähigkeiten. Überdies benötigen sie Zugang zu einem einzelnen, in seiner Kapazität begrenzten Arbeitsgedächtnissystem.

Eine umfassende Analyse von in der Literatur postulierten Merkmalen beider Prozesse wird von Stanovich und Toplak (2012) vorgenommen. Dabei trennen sie diejenigen Eigenschaften, die die beiden Typen von Prozessen nach ihrer Auffassung definieren von denjenigen, die sie lediglich als Korrelate der Prozesse ansehen. Sie kommen zu dem Schluss, dass Autonomie die entscheidende Eigenschaft von Typ 1-Verarbeitungsprozessen darstellt. Dies bedeutet, dass Typ 1-Prozesse bei Vorhandensein der sie auslösenden Stimuli zwingend ablaufen und dann unabhängig von Informationen kognitiver Systeme höherer Ordnung operieren. Hingegen seien die tyischerweise Typ 1-Prozessen zugeordneten Eigenschaften Schnelligkeit und große Verarbeitungskapazität, sowie ihr assoziativer Charakter lediglich als 
Korrelate und nicht als definitorische Merkmale anzusehen. Typ 2-Prozesse haben Stanovich und Toplak zufolge zwei entscheidende Eigenschaften. Zum einen sind sie in der Lage, die Ergebnisse von Typ 1-Prozessen zu unterdrücken, wenn dies z.B. durch die Situation erforderlich erscheint. Zum anderen sind sie in der Lage, Repräsentationen der realen Welt von Repräsentationen als Resultat mentaler Simulationen zu unterscheiden. Diese Fähigkeit wird als kognitives Abkuppeln (cognitive decoupling) bezeichnet (vgl. Stanovich \& Toplak, 2012). Die üblicherweise mit Typ 2-Prozessen assoziierten Eigenschaften langsamer Ablauf, sequentielle Verarbeitung und hohen rechnerischen Anforderungen stellen nach Stanovich und Toplak (2012) hingegen lediglich Korrelate von Typ 2-Verarbeitungsprozessen dar.

Insgesamt kann an dieser Stelle festgehalten werden, dass sich die grundsätzliche Trennung zwischen zwei Arten von Prozessen und zwei Arten von Systemen in einer Vielzahl von Theorien aus unterschiedlichen Bereichen der Psychologie wiederfinden lässt. Darüber hinaus ähneln sich die Eigenschaften der postulierten Prozesse bzw. Systeme über die Ansätze hinweg. Die Analyse von Stanovich und Toplak (2012) stellt in diesem Zusammenhang einen Versuch dar, die Vielzahl der angenommenen Eigenschaften der beiden postulierten Verarbeitungsprozesse auf wenige, elementare Merkmale herunterzubrechen. $\mathrm{Ob}$ sich die von ihnen angenommenen definitorischen Merkmale in der Debatte gegen die Annahmen anderer Autoren (z.B. Evans, 2008, 2011) durchsetzen werden bleibt abzuwarten.

\subsection{Kausales Denken und Zwei-Prozess-Theorien}

Ein zentraler Aspekt der vorliegenden Arbeit ist die Zusammenführung von ZweiProzess-Theorien mit Theorien kausalen Denkens. Dies soll im Folgenden in zwei Schritten geschehen. Zunächst wird untersucht, wie im Rahmen verschiedener Zwei-Prozess-Theorien kausales Denken und Urteilen konzeptualisiert bzw. welchen Prozessen die Verarbeitung kausaler Informationen zugeordnet wird. Dabei wird vor allem die Zuschreibung kausaler Denkprozesse zu den beiden Typen von Prozessen betrachtet. Im anschließenden Abschnitt wird geprüft, ob einzelne Theorien kausalen Denkens Aussagen über zu Grunde liegende kognitive Prozesse machen. Ist dies der Fall, so soll anschließend die Frage nach der Zuordnung dieser Prozesse zu den beiden Prozesstypen geklärt werden.

Bei einer Betrachtung bestehender Zwei-Prozess-Theorien (vgl. Evans, 2008; Stanovich, 2004) fällt auf, dass kausales Denken nur in wenigen dieser Ansätze überhaupt berücksichtigt wird. Dieser Umstand mag zumindest teilweise auch auf die im vorangegangenen Abschnitt eingegangene Debatte über die Berücksichtigung kausaler 
Charakteristika im menschlichen Denken zurückzuführen sein. Daneben ist er jedoch auch ein Indiz dafür, dass verschiedene Forschungszweige trotz thematischer Verwandtschaft oftmals relativ isoliert voneinander arbeiten. Dennoch gibt es Zwei-Prozess-Theorien, die versuchen, die bei der Bearbeitung von Aufgaben mit kausalem Hintergrund ablaufenden Verarbeitungsprozesse einzuordnen. Einige dieser Theorien sollen im Folgenden vorgestellt und im Hinblick auf ihre Zuordnung kausaler Denkprozesse zu den beiden vorgestellten Prozesstypen untersucht werden. Dabei sollen die betrachteten Theorien einheitlich auf ihren Umgang mit vier Aspekten kausalen Denkens untersucht werden: Erstens soll analysiert werden, welche Informationsverarbeitungsprozesse sie bei der Bildung kausaler Urteile annehmen. Hierbei wird bei Vorliegen einer Folge von Ereignissen (z.B. A und B) geschlossen, ob A B verursacht hat oder nicht. Zweitens soll der Umgang mit kausalen Attributionen betrachtet werden, bei denen nach der Beobachtung eines Ereignisses auf dessen Ursache geschlossen wird. Drittens soll untersucht werden, welche kognitiven Prozesse bei (kausalen) Kategorisierungsaufgaben angenommen werden, die in der Regel aus der Präsentation eines Falles und einer anschließenden Kategorisierungsentscheidung bestehen. Viertens sollen die Prozesse beschrieben werden, die nach den verschiedenen Theorien in die Wahl von Interventionen in ein Kausalsystem für eine konkrete Situation bzw. der Abschätzung von Interventionsfolgen involviert sind. Bei allen hier vorgestellten Aspekten kausalen Denkens spielen Vorerfahrungen eine wichtige Rolle. Wurden Aufgaben in der Vergangenheit bereits vielfach ausgeführt, so können sich Assoziationen bilden, die dann eine aktuelle Ausführung der Aufgabe bestimmen können. Neben dem assoziativen Wissen, ist es jedoch auch möglich, kausales Wissen über die Struktur der Aufgabe z.B. durch explizite Wissensvermittlung zu erlangen. Diese zweite Art von Wissen steht im Fokus der vorliegenden Arbeit. Daher werden im empirischen Teil der Arbeit auch Aufgaben verwendet, mit deren Inhalt die Versuchspersonen möglichst wenig vertraut sind.

Es ist wichtig anzumerken, dass die verschiedenen Theorien nicht alle vier zu betrachtenden Aspekte kausalen Denkens explizit berücksichtigen. Berücksichtigt eine Theorie einen Aspekt nicht explizit, wird ausgehend von den in der jeweiligen Theorie angenommenen Charakteristika der beiden Verarbeitungsprozesse eine Zuordnung des betreffenden Aspekts zu einem der beiden Prozesstypen vorgenommen. So wird beispielsweise die Wahl von Interventionen in keiner der Theorien explizit diskutiert. Da diese aber mittels mentaler Simulationen (vgl. Abschnitt 2.4.3.2) durchgeführt wird, kann sie im Rahmen einer Theorie, die mentale Simulationen als Typ 2-Prozess ansieht, ebenfalls diesem Prozesstyp zugeordnet werden. 
Dabei werden zunächst die Ansätze von Smith und DeCoster (2000), sowie Kahneman und Frederick (2002; Kahneman, 2011) betrachtet, die annehmen, dass bestimmte Aspekte kausalen Denkens auch ohne Beteiligung von Typ 2-Prozessen ablaufen können. Anschließend werden die Theorien von Sloman (1996), sowie Strack und Deutsch (2004) vorgestellt, die den Standpunkt vertreten, dass Typ-2-Prozesse notwendig für kausales Denken sind.

\subsubsection{Verarbeitung kausaler Informationen als Typ 1-Prozess}

Die erste vorgestellte Zwei-Prozess-Theorie mit Bezug zum kausalen Denken stammt von Smith und DeCoster (2000). Sie zeigen in ihrem Ansatz Verbindungen zwischen zwei unterschiedlichen Verarbeitungsmodi und zwei unterschiedlichen LangzeitGedächtnissystemen auf. Letztere bestehen aus einer Kombination von Mechanismen, die Erwerb, Beibehaltung und Nutzung von Gedächtnisinhalten ermöglichen und dabei auf Grundlage bestimmter Regeln vorgehen (Sherry \& Schacter, 1987). Ihre Existenz kann nach Ansicht von Smith und DeCoster (2000) als gesichert und evolutionär begründbar angenommen werden. Die Aufgabe des ersten Gedächtnissystems ist das langsame und stufenweise Ansammeln von Informationen, so dass sich über die Zeit Wissen auf Grundlage einer großen Anzahl an Erfahrungen bilden kann. Das zweite Gedächtnissystem erfüllt hingegen die Aufgabe eines schnellen Erwerbs neuer Informationen, die mitunter bereits nach einmaligem Auftreten erinnert werden können. Während das erste, langsame, System somit umfassende, schematische Repräsentationen aufbaut, basiert das zweite System auf detaillierten einzelnen Erfahrungen und deren Verbindung zum Kontext, in denen sie gemacht wurden.

Im Lichte dieser Annahmen unternehmen Smith und DeCoster (2000) den Versuch einer Integration und Interpretation verschiedener sozial- und kognitionspsychologischer Zwei-Prozess-Ansätze, um zu neuen Erklärungen und Vorhersagen in verschiedenen psychologischen Gebieten zu gelangen. Die zwei auf Grundlage der beiden Gedächtnissysteme angenommenen Verarbeitungsmodi werden von Smith und DeCoster wie folgt charakterisiert: Das langsam lernende Gedächtnissystem bildet die Grundlage des assoziativen Verarbeitungsmodus (associative processing mode), der vor allem mittels automatischer Vervollständigung von Mustern (pattern-completion) operiert und Ähnlichkeiten zu Typ 1-Systemen aufweist. In diesem Modus werden aktuelle Beobachtungen oder affektive Reaktionen mit Informationen aus dem Gedächtnis 
angereichert, die aus ähnlichen Situationen wie der aktuell erlebten stammen. Die wichtigsten Eigenschaften des assoziativen Verarbeitungsmodus sind nach Smith und DeCoster:

- eine schnelle und automatische Bereitstellung von Informationen,

- eine reproduktive (d.h. auf Abgleich mit bereits abgespeicherten Informationen basierende) Arbeitsweise, die sich auch gleichzeitig über mehrere Informationen erstrecken kann (parallel constraint satisfaction), sowie

- ein auf allgemeiner Ähnlichkeit zwischen aktuellen Cues und gespeicherten Gedächtnisrepräsentationen basierenden Abruf von Gedächtnisinhalten.

Der zweite von Smith und DeCoster (2000) angenommene Verarbeitungsmodus ähnelt hingegen einem System vom Typ 2. Er arbeitet regelbasiert (rule-based processing mode) und nutzt hierfür symbolisch repräsentiertes und kulturell überliefertes Wissen. Dabei erfolgt die symbolische Repräsentation des Wissens meist in Form von Sprache, die auf die beiden vorgestellten Gedächtnissysteme zurückgreift. Die diesen Verarbeitungsmodus konstituierenden Regeln können ebenfalls in beiden Gedächtnissystemen (in Abhängigkeit der Häufigkeit und Dauer ihrer Nutzung) gespeichert werden. Als wichtigste Eigenschaft des regelbasierten Verarbeitungsmodus nennen Smith und DeCoster die Nutzung symbolisch repräsentierten und willentlich abgerufenen Wissens. Weitere Eigenschaften des regelbasierten Verarbeitungsmodus sind:

- die Möglichkeit, symbolisches Wissen auch mittels einzelner Erfahrungen zu erwerben und für Urteile und Entscheidungen zu nutzen,

- die Fähigkeit, Regeln zu nutzen, anstatt ihnen nur zu entsprechen,

- ein langsam und notwendigerweise sequenziell ablaufender Verarbeitungsprozess, sowie

- die Tendenz zur analytischen Verarbeitung (im Gegensatz zur Verarbeitung aufgrund globaler Ähnlichkeit im assoziativen Verarbeitungsmodus)

Smith und DeCoster (2000) liefern auf Grundlage der von ihnen angenommenen Verarbeitungsmodi Erklärungen für verschiedene Phänomene aus unterschiedlichen Bereichen der Psychologie von denen zwei aufgrund ihrer Relevanz für die vorliegende Arbeit vorgestellt werden sollen. Kategorisierungen werden nach Smith und DeCoster mittels der Vervollständigung von Mustern durchgeführt und sind daher dem assoziativen 
Verarbeitungsmodus zuzuordnen. Es wird davon ausgegangen, dass sich im langsamen Gedächtnissystem Assoziationen zwischen den Merkmalen einer Kategorie bilden, da diese häufig gemeinsam auftreten. Dies führt dazu, dass die Beobachtung einzelner Kategorienmerkmale einem Abruf bzw. einer Rekonstruktion der gesamten MerkmalsKonfiguration einer Kategorie auslösen kann oder, in den Worten von Smith und DeCoster: „the complete pattern is brought forth from a sufficiently distinctive part“ (2000, S. 113). Der Terminologie Evans“ (2009, 2011) folgend können Kategorisierungen somit aufgrund ihrer Verortung im assoziativen Modus als Typ 1-Prozesse verstanden werden.

Kausale Attributionen können nach Smith und DeCoster (2000) ebenfalls auf Grundlage des langsamen Gedächtnissystems und somit im assoziativen Verarbeitungsmodus durchgeführt werden. Damit sind sie ebenfalls zu den Typ 1-Prozessen zu zählen. Der Prozess kann auf zwei unterschiedliche Arten ablaufen: Zum einen können Attributionen auf der Grundlage von Informationen über die Kovariation von Ereignissen vorgenommen werden, die eine wesentliche Grundlage des langsamen Gedächtnissystems bilden. Trat ein aktuell eingetretenes Ereignis in der Vergangenheit häufig gemeinsam mit einer potenziellen Ursache ein, so sind Menschen geneigt, das Ereignis auch im aktuellen Fall auf diese Ursache zu attribuieren (vgl. Kelley, 1973). Darüber hinaus nehmen Smith und DeCoster (2000) an, dass die gespeicherten Assoziationen von Ereignissen im langsamen Gedächtnissystem die kausale Wirkung eines Ereignisses auf ein anderes Ereignis repräsentieren und nicht nur eine einfache Korrelation zwischen den Ereignissen. Diese Annahme steht in Übereinstimmung mit den Untersuchungen zum „Blocking“-Phänomens (Rescorla \& Wagner, 1972; vgl. Abschnitt 2.1.4). Dieses besagt, dass die Stärke einer Assoziation zwischen zwei Ereignissen davon abhängt, in welchem Umfang das eine beteiligte Ereignis spezifische diagnostische Informationen über das Auftreten des anderen Ereignisses liefert (Smith \& DeCoster, 2000). Auf Grundlage dieser im langsamen Gedächtnissystem gespeicherten Information über die kausale Wirkung von Ereignissen kann dann ebenfalls eine Kausalattribution im assoziativen Modus vorgenommen werden.

Problematisch an der Zuordnung kausaler Attributionen zum assoziativen Verarbeitungsmodus ist, dass Smith und DeCoster (2000) ihre Beschreibung kausaler Attributionen aus einer assoziativen Perspektive vornehmen, die die besonderen Charakteristika kausaler Beziehungen unberücksichtigt lässt (vgl. Abschnitt 2.1). Die Zuordnung kausaler Attributionen zum assoziativen Verarbeitungsmodus und die damit einhergehende Klassifikation als Typ 1-Verarbeitungsprozess ist somit kritisch zu betrachten, 
da nicht klar ist, ob die unberücksichtigten Charakteristika ebenfalls im assoziativen Modus verarbeitet werden können.

Kausalurteile werden von Smith und DeCoster (2000) als Beispiele für eine analytische bzw. rationale Art von Urteil aufgeführt. Sie sind in ihrem Ansatz daher eher dem regelbasierten, auf Basis von Typ 2-Prozessen operierenden System zuzuordnen. Ähnlich verhält es sich mit der Simulation der Folgen von Interventionen in ein kausales System: Smith und DeCoster folgen der Auffassung von Clark (1997), indem sie die Planung zukünftiger Handlungen auf der Basis kontrafaktischen Schließens als im regelbasierten System stattfindenden Vorgang einordnen. Es kann angenommen werden, dass an der Planung von Interventionsentscheidungen mittels mentaler Simulation der Folgen verschiedener Optionen vergleichbare Prozesse beteiligt sind. Daher kann die Wahl einer Intervention ebenfalls dem regelbasierten System von Smith und DeCoster (2000) zugeordnet werden, obwohl sie in ihrer Arbeit keine Erwähnung findet.

Kahneman und Frederick (2002) folgen mit ihrer 2-System-Theorie der Unterscheidung zwischen schnellen, assoziativen und langsamen, regelbasierten Prozessen als Grundlage zweier kognitiver Systeme. Sie nutzen die Terminologie von Stanovich (1999) und unterscheiden zwischen einem intuitiven System 1 und einem regelbasierten bzw. reflektierenden System 2.

Die Charakteristika der intuitiven Verarbeitungsprozesse nach Kahneman und Frederick (2002) entsprechen den Merkmalen von Typ 1-Prozessen anderer Zwei-ProzessTheorien: Sie laufen schnell, automatisch und mühelos ab und sind assoziativer Natur. Zudem können mehrere intuitive Prozesse parallel ablaufen. Der Verarbeitungsprozess selbst ist dem Bewusstsein nicht zugänglich, lediglich das Ergebnis ist bewusstseinsfähig. Der Prozess des Musterabgleichs (pattern matching) dient als Beispiel eines typischen intuitiven Prozesses. Kahneman und Frederick verstehen intuitives Denken als eine Art Erweiterung perzeptueller Verarbeitungsprozesse auf nicht notwendigerweise anwesende Beurteilungsobjekte. So können die zu beurteilenden Objekte beispielsweise auch durch Sprache evozierte mentale Repräsentationen sein. Die beim intuitiven Denken benutzten Repräsentationen weisen die Eigenschaften Konkretheit und Spezifität auf. Darüber hinaus besitzen sie einen affektiven Gehalt und beinhalten kausale Tendenzen. Somit schreibt die Theorie von Kahneman und Frederick dem intuitiven System 1 eine Fähigkeit zur Berücksichtigung kausaler Informationen zu. Dadurch unterscheidet sie sich z.B. von der 2-System-Theorie Slomans (1996), der die Verarbeitung kausalen Wissens als einen regelbasierten Prozess versteht. Als 
Beispiel einer intuitiven Kategorisierung (intuitive categorical prediction) kann die sogenannte conjunction fallacy dienen, bei der eine das Auftreten einer Konjunktion zweier Ereignisse intuitiv wahrscheinlicher als das Auftreten nur eines der an der Konjunktion beteiligten Ereignisse eingeschätzt wird. Kahneman und Frederick (2002) erklären dieses Phänomen mit der Aktivierung kausaler Schemata und deren Nutzung bei der Bildung des intuitiven Urteils.

Kahneman (2011) erweitert die Sichtweise, nach der das intuitive System 1 in Bezug auf die Generierung von Kausalurteilen eine wichtige Rolle spielt. Dabei geht er von der Annahme aus, dass Menschen danach streben, Vorgänge in der Welt kausal und kohärent zu erklären. Um dies zu gewährleisten kommt dem Aufspüren kausaler Verbindungen eine zentrale Rolle zu, die das System 1 über einen automatisch ablaufenden Prozess übernimmt. Dabei können die zu identifizierenden Verbindungen sowohl auf physikalischer als auch auf intentionaler Kausalität basieren. Die Zuordnung kausaler Urteilsprozesse zum System 1 lässt sich nach Kahneman auch evolutionär begründen: Die standardmäßige Annahme kausaler Beziehungen in der Welt ermöglichte es unseren Vorfahren, Veränderungen in der Umwelt schnell als Abweichungen von gewohnten kausalen Mustern zu erkennen und entsprechend zu reagieren. Sie ist somit ein Teil einer von unseren Vorfahren vererbten allgemeinen Vigilanz. Kausale Attributionen werden von Kahneman ebenfalls als eine Art Routineaufgabe des Intuitiven Systems verstanden, die automatisch bei der Wahrnehmung von Ereignissen durchgeführt werden.

Zur Unterstützung seiner Annahmen führt Kahneman (2011) Belege aus dem Bereich der kognitiven Urteilsverzerrungen an. Die Neigung von Menschen, zufällige Ereignissequenzen oder die Regression zur Mitte kausal zu interpretieren, werden als Beispiele für den intuitiven und automatischen Charakter kausaler Attributionen angeführt. Hingegen ist das Schließen auf Grundlage statistischer Regeln ein mühevoller Prozess, der nur auf der Basis vorhandenen statistischen Wissens im deliberierenden bzw. reflektierenden System 2 durchgeführt werden kann.

Das reflektierende Denken steuert nach Kahneman und Frederick (2002) die Bearbeitung unbekannter Aufgaben, die Verarbeitung abstrakter Konzepte und die willentliche Anwendung von Regeln. Seine Prozesse werden als kontrolliert, aufwändig und deduktiv charakterisiert. Es verarbeitet Informationen langsam und seriell. Die Verarbeitungsprozesse sowie das Ergebnis des reflektierenden Denkens sind dem Bewusstsein zugänglich. Somit entsprechen die Merkmale der Prozesse des reflektierenden 
Denkens den Typ 2-Prozessen. Die Planung von Interventionen in ein kausales System kann auch bei Kahneman und Frederick als im reflektierenden System ablaufender Vorgang interpretiert werden, da sie kontrolliert, langsam, seriell und deduktiv erfolgt.

Kahneman und Frederick (2002) sehen eine klare Hierarchie zwischen intuitivem und reflektierendem Denken. Intuitives Denken generiert schnelle Antworten auf aktuell vorliegende Urteilsprobleme. Die Qualität dieser Antworten wird vom reflektierenden System überwacht. Je nach Ergebnis der Überprüfung akzeptiert, modifiziert oder verwirft es die Vorschläge des intuitiven Systems. Nur wenn eine intuitive Antwort ohne wesentliche Modifikationen übernommen wird, kann man von einem intuitiven Urteil sprechen. Die Rolle der beiden Systeme bei der Urteilsbildung hängt nach Kahneman und Frederick von verschiedenen Einflussfaktoren ab. So können bestimmte Charakteristika der Aufgabe wie bspw. Restriktionen bezüglich der Verarbeitungsdauer dazu führen, dass der kontrollierende Einfluss des reflektierenden Systems zurückgeht. Zudem üben individuelle Eigenschaften der Urteilenden wie momentane Stimmung, Intelligenz oder Erfahrung mit der Art der Aufgabe Einflüsse auf die Rolle der beiden Systeme im Entscheidungsprozess aus.

Zusammenfassend kann festgestellt werden, dass beide hier vorgestellten Theorien annehmen, dass Typ 1-Prozesse in bestimmte Aspekte kausalen Denkens involviert sind. Sowohl Smith und DeCoster (2000) als auch Kahneman und Frederick (2002; Kahneman, 2011) sehen kausale Attributionen und Kategorisierungen als Aufgaben an, die mit Typ 1Prozessen bearbeitet werden können. Für Kahneman und Frederick können darüber hinaus auch Kausalurteile auf der Basis von Typ 1-Prozessen gebildet werden, während diese bei Smith und DeCoster Typ 2-Prozesse zu beinhalten scheinen. Die Planung von Interventionen in ein kausales System kann hingegen bei beiden Ansätzen als im Typ 2-System stattfindend interpretiert werden. Es ist wichtig anzumerken, dass für beide Theorien gilt, dass diejenigen Vorgänge im Bereich des kausalen Denkens, die im vorangegangenen Abschnitt als auf Typ 1-Prozessen basierend klassifiziert wurden auch bewusst und willentlich (also als Typ 2Prozesse) durchgeführt werden können. Es ist das angenommene Vermögen von Typ 1Prozessen, kausale Attributionen und Kategorisierungen (sowie im Fall der Theorie von Kahneman und Frederick auch Kausalurteile) vorzunehmen, das sie von den im folgenden Abschnitt vorgestellten Ansätzen von Sloman (1996), sowie Strack und Deutsch (2004) unterscheidet. 


\subsubsection{Verarbeitung kausaler Informationen als Typ 2-Prozess}

Sloman (1996) stellt eine Zwei-System-Theorie für den Bereich des schlussfolgernden Denkens auf. Er unterscheidet zwischen zwei kognitiven Systemen, einem assoziativen und einem regelbasierten. Das assoziative System gelangt nach Sloman auf der Grundlage von Ähnlichkeit und Kontiguität zu Schlüssen. Seine Arbeitsweise besteht in der Einteilung von Perzeptionen in verschiedene Cluster auf der Basis statistischer Regularitäten in der Umwelt. Die Regularitäten äußern sich in wahrgenommenen Ähnlichkeiten und temporären Strukturen zwischen verschiedenen Objekten. Den Perzeptionen eventuell zu Grunde liegende kausale oder mechanische Strukturen werden vom assoziativen System hingegen nicht berücksichtigt. Nach Ansicht Slomans repräsentieren Assoziationen Strukturen nicht über die Berechnung von Relationen, sondern direkt. Darüber hinaus genügt es, wenn eine Ähnlichkeitsstruktur implizit über eine Menge von Assoziationen repräsentiert wird. Eine explizite Repräsentation ist nicht notwendig.

Im Gegensatz versucht das regelbasierte System nach Sloman (1996) die logische, hierarische und kausale Struktur der Umwelt zu erfassen und folgt dabei den rechnerischen Prinzipien der Produktivität und Systematik. Unter Produktivität ist dabei die Eigenschaft von regelbasierten Systemen zu verstehen, durch die Kombination von Regeln eine unbegrenzte Zahl von Propositionen generieren zu können. So kann beispielsweise in der Arithmetik immer wieder eine neue Zahl generiert werden, indem zur größten Zahl aus einer bestehenden Menge von Zahlen eine feste Größe (z.B. 1) addiert wird. Das Prinzip der Systematik von Regeln charakterisiert Sloman wie folgt: Besitzt eine Regel die Fähigkeit, bestimmte Fakten zu kodieren (z.B. John liebt Mary), so impliziert dies, dass diese Fähigkeit auch auf andere Fakten übertragen werden kann (z.B. Mary liebt John). Eine weitere Eigenschaft von Regeln ist ihre Abstraktheit, die ihre Anwendbarkeit auf alle Aussagen, die eine bestimmte symbolische Struktur aufweisen, ermöglicht. Sloman betont, dass das regelbasierte System Regeln explizit folgt und ihnen nicht nur entspricht. Darüber hinaus begrenzt er den Anwendungsbereich des regelbasierten Systems auf schlussfolgerndes Denken.

Zusammenfassend lassen sich die postulierten Systeme folgendermaßen charakterisieren (vgl. Sloman, 1995, Tabelle 1): Das assoziative System basiert auf den Prinzipien der Ähnlichkeit und Kontiguität und bezieht sein Wissen aus persönlichen Erfahrungen. Die verwendeten Repräsentationen bestehen aus konkreten und generischen Konzepten, Bildern, Stereotypen und Merkmalen, die über Assoziationen miteinander verbunden sind. Die Verarbeitung erfolgt automatisch und ist in der Regel reproduktiv, es 
kann jedoch auch zu ähnlichkeitsbasierten Generalisierungen kommen. Es werden Integrationen über alle Merkmale unter Berücksichtigung vorgegebener Bedingungen (constraint satisfaction) vorgenommen. Als Beispiele kognitiver Funktionen, die im assoziativen System ablaufen nennt Sloman Intuition, Phantasie, Kreativität, Imagination, visuelles Erkennen und die Operationen des assoziativen Gedächtnisses. Das assoziative System kann aufgrund der vorgestellten Merkmale auch als Typ 1-System verstanden werden.

Das regelbasierte System arbeitet nach dem Prinzip der Manipulation von Symbolen und bezieht sein Wissen aus Sprache, Kultur und formalen Systemen. Die verwendeten Repräsentationen bestehen aus konkreten, generischen und abstrakten Konzepten, abstrahierten Merkmalen oder zusammengesetzten Symbolen zwischen denen kausale, logische oder hierarchische Verbindungen bestehen. Die Verarbeitung erfolgt produktiv und systematisch und beinhaltet die Abstraktion relevanter Merkmale. Darüber hinaus ist sie strategisch (im Gegensatz zur automatischen Verarbeitung des assoziativen Systems). Sloman (1996) ordnet die kognitiven Funktionen Deliberation, Erklärung, formale Analyse, Verifikation, Zuschreibung von Zweck (ascription of purpose) und die Operationen des strategischen Gedächtnisses dem regelbasierten System zu. Das regelbasierte System kann somit auch als ein System vom Typ 2 charakterisiert werden.

Die beiden Systeme interagieren nach Ansicht Slomans normalerweise bei der Bewältigung einer Aufgabe. Im Falle eines neuen Problems kann es jedoch vorkommen, dass beide Systeme unabhängig voneinander nach einer Lösung suchen und $\mathrm{zu}$ sich widersprechenden Ergebnissen gelangen. Sloman postuliert keine klare Hierarchie zwischen den Systemen. Daher ist es nicht immer einfach, das System zu identifizieren, das eine gegebene Antwort generiert hat. Anhand der Bewusstseinsinhalte kann die Quelle der Antwort jedoch manchmal ausgemacht werden, da im assoziativen Systems meist nur die Antwort bewusstseinsfähig ist, während im regelbasierten System auch der zur Antwort führende Prozess bewusst abläuft. Da beide Systeme jedoch auch zu derselben Antwort gelangen können und es zudem auch nicht-assoziative Prozesse gibt, die außerhalb der bewussten Wahrnehmung ablaufen (z.B. bei Intuitionen von Experten) ist die Identifikation mittels Bewusstseinsinhalten anfällig für Fehler.

Ein wichtiges Anwendungsfeld der Zwei-System-Theorie Slomans (1996) stellt die Frage nach der mentalen Repräsentation von Kategorien dar. Sloman stellt in diesem Zusammenhang zwei Klassen von Ansätzen gegenüber: Die erste Klasse von Ansätzen geht davon aus, dass ähnlichkeitsbasierte Prozesse die Basis von Kategorisierungen bilden. Als 
Beispiele führt Sloman Prototyp- und Expemplar-Kategorisierungsmodelle an. Dabei wird ein Prototyp einer Kategorie als diejenige Instanz verstanden, die die höchste Ähnlichkeit zu den Mitgliedern der eigenen Kategorie und die niedrigste Ähnlichkeit zu Mitgliedern anderer Kategorien aufweist. Bei den Expemplar-basierten Ansätzen wird hingegen davon ausgegangen, dass Menschen alle von ihnen beobachteten Instanzen im Gedächtnis speichern und nur dann auf Grundlage ihrer Ähnlichkeit gruppieren, wenn sie durch eine Kategorisierungsaufgabe dazu aufgefordert werden.

Eine zweite Klasse von Ansätzen wird von Sloman (1996) unter dem Begriff der Theorie-basierten Kategorisierung zusammengefasst, da sie laienhafte Theorien (lay theories) als Grundlage von Kategorisierungen annehmen. Diese Ansätze betonen, dass Ähnlichkeitsbeziehungen allein nicht ausreichend sind, um Kategorisierungen zu erklären. Beispielsweise können ähnlichkeitsbasierte Ansätze nicht erklären, welche Objekteigenschaften aufgrund ihrer bestehenden Beziehungen zu Objekten oder Kategorien auf andere Objekte oder Kategorien projiziert werden können. Nach Murphy (1993, zit. n. Sloman, 1996) sind Menschen bei bestimmten Eigenschaften von Objekten eher bereit, diese auf andere Objekte derselben Kategorie zu generalisieren. Dieses Phänomen erfordert eine bestimmte Art von Wissen, das als Theoriewissen über die Übertragbarkeit von Objektmerkmalen in einem spezifischen Kontext aufgefasst werden kann. Ein weiteres Problem ähnlichkeitsbasierter Ansätze identifiziert Sloman darin, dass sie die Kontextabhängigkeit von Ähnlichkeiten nicht berücksichtigen. Als Beispiel nennt er den Fall eines mechanischen Affen, der in einem Kontext aufgrund seines Aussehens als ähnlich zu einem echten Affen, in einem anderen Kontext aber aufgrund seiner inneren Struktur als ähnlich zu einem mechanischen Pferd eingeschätzt wird.

Darüber hinaus können einfache Ähnlichkeitsstrukturen die Verwendung von Konzepten nicht erklären, die als zentrale Elemente von (Laien-)Theorien verstanden werden können. Diese Theorien dienen wiederrum der Erklärung beobachteter Kausalrelationen (Sloman, 1996). Je besser eine Theorie die Angemessenheit der Wahl einer bestimmten Kategorie erklären kann, desto eher wird diese Kategorie ausgewählt. Sloman ordnet Theoriebasierte Kategorisierungen dem regelbasierten System zu, da sie Erklärungen nutzen, die dem regelbasierten Denken entstammen und die Nutzung von (Laien-)Theorien die Anwendung einer Regel nahelegt. Zudem kann ausgeschlossen werden, dass Theorie-basierte Kategorisierungen im assoziativen System ablaufen, da dieses von der Annahme ausgeht, dass die Verarbeitung auf der Basis von Ähnlichkeit abläuft. Zur Stützung seiner Annahmen stellt 
Sloman (1996) Studien von Rips (1989) und Smith und Sloman (1994) vor. Diese Arbeiten zeigen, dass Ähnlichkeit zwischen einem Stimulus und einer Kategorie nicht zwangsläufig mit einer Zuordnung des Stimulus zu dieser Kategorie einhergeht. Wie Sloman (1996) betont, laufen Kategorisierungsprozesse jedoch nicht immer explizit regelbasiert ab, sondern nur dann wenn die Voraussetzungen (wie die Existenz eindeutiger Regeln oder Bedingungen, die das bewusste Nachdenken fördern) hierfür günstig sind. Kategorisierungen können somit auch auf der Grundlage von ähnlichkeitsbasierten, assoziativen Prozessen vorgenommen werden.

Mit Blick auf die vorliegende Arbeit wird deutlich, dass Sloman (1996) die Verarbeitung kausaler Beziehungen (z.B. im Rahmen Theorie-basierter Kategorisierungen) dem regelbasierten System zuordnet, dessen Charakteristika weitgehend mit denen der Prozesse vom Typ 2 übereinstimmen. Ähnlich wie Kahneman (2011) haben Menschen nach Ansicht Slomans (1996) ein Bedürfnis, ihre Umwelt auf der Basis kausaler Beziehungen (bewusst) zu verstehen. Während Kahneman (2011) diese Annahme als Basis seiner Argumentation für die Existenz von ausschließlich auf Typ 1-Prozessen basierenden kausalen Denkvorgängen nutzt, geht Sloman (1996) davon aus, dass beim kausalen Denken und Urteilen ein regelbasierter (Typ 2-) Prozess abläuft, es sei denn, es bestehen bereits durch häufige Erfahrungen gebildete Assoziationen, die auch mit Typ 1-Prozessen verarbeitet werden können. Außerdem können kausale Strukturen nur über Regeln und damit im regelbasierten System erfasst werden. Daher kann davon ausgegangen werden, dass für Kausalurteile und kausale Attributionen Prozesse vom Typ 2 erforderlich sind. Als weiterer Beleg für diese Annahme kann die Beschreibung der Arbeitsweise des assoziativen Systems dienen: „Rather than trying to reason on the basis of an underlying causal or mechanical structure, it constructs estimates based on underlying statistical structure“ (Sloman, 1996, S.4).

Kausales Wissen kann nach Sloman (1996) Ähnlichkeitsrelationen und damit auch assoziative Urteilsprozesse beeinflussen. So steigt beispielsweise die Repräsentativität einer zu beurteilenden Aussage, wenn diese im Einklang mit bestehendem Kausalwissen über den $\mathrm{zu}$ beurteilenden Sachverhalt steht. Sloman erläutert diesen Vorgang am Beispiel einer conjunction fallacy im Bereich der Kategorisierung: Versuchspersonen schätzten die Wahrscheinlichkeit, dass John P. einen Angestellten umgebracht hat als geringer ein als die Wahrscheinlichkeit, dass John P. einen Angestellten umgebracht hat, um zu verhindern, dass dieser mit der Polizei spricht. Sloman folgert daraus, dass die Ähnlichkeit einer Handlung (hier: der Mord) zu den von einem Mörder erwarteten Handlungen durch die Einführung eines 
Motivs bzw. einer Ursache für die Handlung (hier: Verhindern, dass der Angestellte mit der Polizei spricht) erhöht wird. Diese Folgerung setzt zwei Bedingungen voraus: Erstens muss ein Verständnis dafür vorliegen, dass der Mord an dem Angestellten zur Verhinderung eines Gesprächs mit der Polizei eine Kausalrelation zwischen Motiv und Mord darstellt. Da dieses Verständnis eine kausale Analyse beinhaltet, muss Sloman zufolge auch mindestens eine Regel (und somit ein Typ 2-Prozess) am Verständnisprozess beteiligt sein. Zweitens ist eine Entscheidung nötig, dass eine Beschreibung, die ein Motiv für den Mord beinhaltet dem „Standardmodell“ eines Mordes ähnlicher ist als eine Beschreibung ohne Motiv. Dieser Entscheidungsprozess ist nach Auffassung Slomans assoziativer Natur (Typ 1-Prozess). Insgesamt beinhaltet die Berücksichtigung kausalen Wissens bei einer Kategorisierung somit immer auch mindestens einen regelbasierten (Typ 2-) Prozess.

Obwohl die Planung von Interventionen in ein kausales System auch bei Sloman (1996) nicht explizit erwähnt wird liegt es nahe, diesen Vorgang dem regelbasierten System zuzuordnen. Der Grund hierfür liegt in der Ähnlichkeit der Charakteristika des regelbasierten Systems und den Anforderungen, die Planungen von Interventionen an den kognitiven Apparat stellen. Neben der bereits erwähnten Berücksichtigung kausaler Beziehungen ist hier vor allem die produktive, systematische und strategische Art der Verarbeitung im regelbasierten System zu nennen. Zudem lassen sich die einige der Eigenschaften des assoziativen Systems (automatischer Ablauf, Berücksichtigung von Assoziationen, reproduktive Verarbeitung) kaum mit den Anforderungen der Planung von Interventionen vereinbaren. Lediglich bei Interventionen, die der ausführenden Person bereits so gut bekannt sind, dass assoziative Verknüpfungen angelegt wurden, kann es zu einer automatischen Ausführung ohne die Beteiligung von Typ 2-Prozessen kommen. Neue Handlungen bzw. Interventionen erfordern hingegen Typ 2-Prozesse, um ihre Folgen planen zu können.

Ein weiterer Ansatz, der kausales Denken als Prozess eines Typ-2-Systems versteht stammt von Strack und Deutsch (2004). Im Fokus ihres 2-System-Modells steht das Verhalten in sozialen Situationen. Die Autoren gehen davon aus, dass dieses als eine gemeinsame Funktion der parallel ablaufenden Prozesse eines reflektierenden (reflective) und eines impulsiven (impulsive) Systems verstanden werden kann. Dabei erzeugt das reflektierende System Verhalten auf Grundlage von Wissen über Fakten und Werte. Es kann als eine Art Aufmerksamkeit erfordernder, temporärer Speicher mit begrenzter Kapazität charakterisiert werden, in dem vorgehaltene Repräsentationen verblassen, wenn sie nicht aktiv gehalten werden. Die Funktion des reflektierenden Systems besteht in der Erzeugung deklarativen 
Wissens durch die Zuordnung von perzeptuellem Input zu semantischen Kategorien. Des Weiteren haben Repräsentation im reflektierenden System nach Strack und Deutsch die wichtige Eigenschaft, dass sie flexibel generiert und modifiziert werden können. Dadurch wird das reflektierende System in die Lage versetzt, verschiedene Aufgaben wie schlussfolgerndes Denken, Planungen und mentale Simulationen durchzuführen. Das impulsive System wird im Modell von Strack und Deutsch hingegen als einfaches assoziatives Netzwerk verstanden. Es verfügt über die Eigenschaften eines Langzeitgedächtnisses und erzeugt Verhalten über assoziative Verknüpfungen und motivationale Orientierungen. Insgesamt ähnelt somit das reflektierende System in seinen Eigenschaften einem Typ 2-System, während das impulsive System Ähnlichkeiten mit einem Typ 1-System aufweist.

Um die in diesem Abschnitt betrachteten Aspekte kausalen Denkens zu den im Ansatz von Strack und Deutsch (2004) postulierten Systemen zuordnen zu können ist es wichtig, den Ablauf der Generierung propositionaler Repräsentationen zu verstehen. Zunächst wird davon ausgegangen, dass ein perzeptueller Input bestimmte, assoziativ verknüpfte Konzepte im impulsiven System aktiviert. Die in die Proposition eingehenden Elemente (mindestens ein Konzept und das zu benutzende Beziehungsschema) werden dann aus dem impulsiven System abgerufen. Anschließend wird aus den Elementen im reflektierenden System eine propositionale Repräsentation erzeugt, die die Beschaffenheit der Welt bzw. des Organismus wiederspiegelt. Darüber hinaus können auf Basis einer propositionalen Repräsentation mittels Anwendung von Regeln Inferenzen gebildet werden. Strack und Deutsch illustrieren den Ablauf am Beispiel einer propositionalen Kategorisierungsaufgabe (z.B. „Ist A ein B?“). Nach der Wahrnehmung von A werden mit A im impulsiven System verknüpfte Konzepte aktiviert. Zusätzlich wir das Beziehungsschema für Kategorienzugehörigkeit (,,ist ein“), sowie das Label der Kategorie („B“) aus dem impulsiven System abgerufen. Im reflektierenden System werden die Elemente zu einer propositionalen Repräsentation verknüpft (z.B. „A ist ein B“), die für Inferenzen genutzt werden kann. Der Ablauf bei der Bildung von kausalen Attributionen und Kausalurteilen (letztere werden von Strack und Deutsch nicht explizit erwähnt) folgt dem selben Muster. Der wesentliche Unterschied besteht in der Aktivierung anderer Beziehungsschemata (nämlich solchen für Verursachungen). Es kann angenommen werden, dass die Planung von Interventionen in ein kausales System ebenfalls unter Beteiligung des reflektierenden Systems vonstatten geht, da die für diesen Vorgang zentralen mentalen Simulationen (vgl. Abschnitt 2.4.3.2) von Strack und Deutsch als typische Aufgabe für dieses System angeführt werden. 
Zusammenfassend kann festgestellt werden, dass die betrachteten Zwei-ProzessTheorien unterschiedliche Standpunkte bezüglich der Zuordnung der betrachteten Aspekte kausalen Denkens zu den von ihnen postulierten Verarbeitungssystemen einnehmen. Für zwei der betrachteten Theorien (Smith \& DeCoster, 2000; Kahneman \& Frederick, 2002) können bestimmte kausale Denkprozesse in Typ 1-Systemen ablaufen, während die Theorien von Sloman (1996), sowie Strack und Deutsch (2004) von der Notwendigkeit der Operation von Typ 2-Prozessen (und damit der Zuordnung zu Typ 2-Systemen) bei kausalen Denkvorgängen ausgehen. Das Ergebnis der vorgenommenen Analyse ist jedoch mit folgender Anmerkung zu versehen: Kausalurteile, kausale Attributionen, Kategorisierungen und die Wahl einer Intervention können unter Umständen nach den betrachteten Theorien auch ohne Beteiligung von Typ 2-Prozessen ablaufen. Dies ist dann der Fall, wenn vielfältige (Lern-)Erfahrungen in der Vergangenheit zur Bildung von assoziativen Verbindungen im Langzeitgedächtnis geführt haben. Auf Grundlage dieser Assoziationen kann bei Vorliegen der entsprechenden Rahmenbedingungen eine Entscheidung automatisch und unbewusst im Typ 1-System getroffen werden. Hat sich beispielsweise eine bestimmte Intervention in einer bestimmten Situation in der Vergangenheit bereits häufig bewährt (z.B. das schnelle Entfernen eines Topfes mit überkochender Milch von der heißen Herdplatte) und steht eine neuerliche Interventionsentscheidung in einer solchen Situation an (das Volumen der im Topf befindlichen Milch vergrößert sich rapide), so kann diese auch ohne die Beteiligung bewusster Typ 2-Prozesse stattfinden. Ist die Situation oder die Aufgabe hingegen neu bzw. unbekannt (d.h. es existieren keine assoziativen Verbindungen im Gedächtnis), so sind Prozesse vom Typ 2 für die dann notwendig Planung der Intervention und die Abschätzung der Interventionsfolgen erforderlich. Eine System 1-Entscheidung, die auf durch instrumentelles Lernen hervorgerufene assoziative Verbindungen beruht, unterscheidet sich wesentlich von einer (System 1- oder System 2-) Entscheidung auf der Grundlage kausalen Lernens. Assoziative Repräsentationen kausalen Wissens berücksichtigen nicht sämtliche Charakteristika kausaler Beziehungen (vgl. Abschnitt 2.1) und können nicht für die Vorhersage unbekannter Phänomene in einer kausalen Struktur genutzt werden. Daher handelt es sich bei System 1-Entscheidungen auf der Grundlage assoziativer Verbindungen genau genommen auch nicht um Aspekte kausalen Denkens, die im Fokus dieses Abschnittes stehen.

Es muss überdies betont werden, dass keine der Theorien kausales Denken mit allen seinen in Abschnitt 2.1 vorgestellten Charakteristika betrachtet. Generell steht die Betrachtung kausaler Denkvorgänge bei keiner der vorgestellten Theorien im Zentrum des 
Interesses, sondern dient in der Regel der Illustration eines Prozesses (der je nach Theorie vom Typ 1 oder Typ 2 sein kann). In diesem Zusammenhang kann auch der Umstand verstanden werden, dass die überwiegende Mehrheit bestehender Zwei-Prozess-Theorien Kausalität gänzlich unberücksichtigt lässt.

\subsection{Berücksichtigung kognitiver Prozesse in kausalen Theorien}

\subsubsection{Einleitung}

Nachdem im vergangenen Abschnitt verschiedene Zwei-Prozess-Theorien im Hinblick auf die Berücksichtigung kausaler Informationsverarbeitung untersucht wurden, sollen im Folgenden kausale Theorien im Zentrum des Interesses stehen. Dabei soll ein besonderes Augenmerk auf der Frage liegen, ob sie Annahmen über die Art der kausalen Informationsverarbeitung zu Grunde liegenden kognitiven Prozesse machen und - wenn ja welchen Typ von Verarbeitungsprozess (Typ 1 oder Typ 2) sie bei der Bearbeitung der von ihnen untersuchten Phänomene annehmen. Zuerst sollen kausale Theorien betrachtet werden, die sich mit der Vorstellung eines unbewussten, intuitiven Einflusses von Kausalität bzw. bestehendem kausalen Wissen auf Urteile und Wahrnehmungen vereinbaren lassen. In diesem Zusammenhang werden Ansätze zur perzeptuellen Kausalität (Michotte, 1963), Urteilsverzerrungen (Tversky \& Kahneman, 1980; White, 2006a), sowie Kategorisierungsurteilen (Luhmann, Ahn \& Palmeri, 2006).betrachtet. Zweitens werden kausale Theorien vorgestellt, die nahelegen, dass beim kausalen Urteilen und Entscheiden die Beteiligung von Typ 2-Prozessen notwendig ist. Hierzu zählen Theorien, die kausale Urteile in Analogie zum logischen Schließen verstehen (Cheng \& Holyoak, 1985; Cummins, Lubart, Olksnis und Rist, 1991; Goldvarg \& Johnson-Laird, 2001), und solche, die annehmen, dass mentale Simulationen einen integralen Bestandteil des Urteils- und Entscheidungsprozesses (Lagnado, 2011; Meder, Gerstenberg, Hagmayer \& Waldmann, 2010; Sloman \& Hagmayer, 2006; Sloman \& Lagnado, 2005) bilden. Drittens werden kausale Theorien vorgestellt, die für die Beteiligung beider Prozesstypen bei kausalen Urteilen und Entscheidungen plädieren. Viertens sollen abschließend einige Theorien Erwähnung finden, die keine Aussage bezüglich der Prozesse machen, die der kausalen Informationsverarbeitung zu Grunde liegen. 


\subsubsection{Theorien der kausalen Intuition}

\subsubsection{Perzeptuelle Kausalität}

Ein Phänomen auf dem Gebiet der Kausalität, das sich gut mit der Vorstellung von kausalem Denken auf der Grundlage von Typ 1-Prozessen vereinbaren lässt ist die perzeptuelle Kausalität. Die Forschung zu diesem Thema geht größtenteils auf die Studien von Albert Michotte (1963) zurück, der zeigen konnte, dass Menschen spontan Kausalurteile fällen wenn sie bestimmte Ereignisse beobachten. Diese Ereignisse können sehr abstrakter Natur und damit untypisch für die tägliche Lebensumwelt von Versuchspersonen sein. Das wohl bekannteste Beispiel im Bereich der perzeptuellen Kausalität ist der sog. launching effect (Michotte, 1963): Den Versuchspersonen werden zwei Objekte (z.B. Quadrate) auf einer Fläche (bzw. einem Bildschirm) präsentiert, von denen sich eines bewegt während das andere unbewegt ist. Dabei bewegt sich das erste Objekt in Richtung auf das unbewegte Objekt. In dem Moment, in dem sich die beiden Objekte berühren stoppt das erste Objekt seine Bewegung, während das zweite Objekt beginnt, sich zu bewegen. Dabei bewegt es sich in der gleichen Richtung wie zuvor das erste Objekt (d.h. von diesem weg). Dieser Vorgang wird von einem großen Anteil der Betrachter als eine Art “Anstoßen” des zweiten Objektes durch das erste Objekt wahrgenommen (launching). Der beobachtete Prozess wird somit als kausal interpretiert. Da es sich bei den beobachteten Objekten jedoch lediglich um Abbildungen (die zudem nicht einmal eine Ähnlichkeit zu realen Objekten aufweisen müssen) und nicht um physische Objekte (bei denen das erste Objekt seinen Impuls auf das zweite Objekt überträgt) handelt, wird der Vorgang des „Anstoßens“ (und damit der gesamte Kausalprozess) lediglich inferiert. Neben dem launching effect gibt es noch weitere Phänomene im Bereich der wahrgenommenen Kausalität (z.B. entraining oder triggering; vgl. Scholl \& Tremoulet, 2000). Diese Effekte werden allerdings in der vorliegenden Arbeit nicht näher betrachtet, da sie keine zusätzlichen Informationen für die hier interessierende Fragestellung liefern.

Eine kontrovers diskutierte Frage besteht in der Beschaffenheit und Herkunft der kognitiven Prozesse, die für perzeptuelle Kausalität verantwortlich sind (für Überblicksarbeiten vgl. Danks, 2009; Rips,2008, 2011). In seinem kritischen Überblick der diese Frage diskutierenden Literatur macht Rips (2011) zwei gegensätzliche Standpunkte aus. Zum einen gibt es die auf Michotte (1963) zurückgehende Vorstellung einer Art von Kausalitäts-Detektor, der angeboren ist bzw. sich aus angeborenen Strukturen früh in der Entwicklung ausbildet. Diesen Detektor versteht Rips (2011) als ein domänenspezifisches 
Input-Modul im Sinne Fodors (1983). Er ist in der Lage, auf Grundlage seines perzeptuellen Inputs, niedrigstufige Inferenzprozesse (low-level inferences) durchzuführen, wobei er unabhängig von Erwartungen oder vorhandenen Gedächtnisinhalten (also informationell eingekapselt bzw. bottom-up) operiert. Dabei wird seine Arbeitsweise nicht durch Lernerfahrungen beeinflusst. Es ist jedoch möglich, dass er sich im Rahmen von Entwicklungsprozessen verändert. Das Ergebnis der Informationsverarbeitung im KausalitätsDetektor ist eine Repräsentation der inferierten kausalen Interaktion.

Vertreter eines alternativen Standpunktes (z.B. Weir, 1978; White, 2006b) gehen hingegen davon aus, dass kausale Schemata eine zentrale Rolle bei der Wahrnehmung von Kausalität spielen (Rips, 2011). Kausale Schemata sind als abstrakte Repräsentationen im Langzeitgedächtnis gespeichert und werden schnell und automatisch mittels räumlicher, zeitlicher oder anderer Cues mit einer aktuellen Beobachtung (z.B. eines launching events) abgeglichen. Das Ergebnis eines solchen Abgleichs besteht in der Interpretation einer beobachteten Interaktion als kausal bzw. nicht kausal. Der Prozess dieser Interpretation findet typischerweise außerhalb der bewussten Wahrnehmung statt und erfolgt schnell und mühelos. Lediglich bei unerwarteten Ereigniskomponenten wird eine bewusste, langsame und aufwändige (Typ 2-)Verarbeitung vorgenommen (vgl. Weir, 1978). Das Ergebnis ist dem Arbeitsgedächtnis zugänglich, auch wenn es mittels unbewusster Informationsverarbeitung zustande gekommen ist. Am Phänomen der perzeptuellen Kausalität ist dem Schema-Ansatz zufolge somit eine Kombination von domänenübergreifenden top-down- (Abgleich mit im Langzeitgedächtnis gespeicherten kausalen Schemata) und bottom-up-Prozessen (Verarbeitung perzeptueller Informationen) beteiligt. Kommt es zu keinen unerwarteten Ereignissen bilden Typ 1-Prozesse die Grundlage der Interpretation.

Die Debatte um die Existenz eines (angeborenen) Moduls für kausale Wahrnehmungen lässt sich mit Danks (2009) wie folgt zusammenfassen: Es gibt auf der einen Seite einige Hinweise, die für die Existenz eines solchen Moduls sprechen. Kausale Wahrnehmungen erfolgen fast ohne Zeitverzug, automatisch, unwillkürlich und informationell eingekapselt (informationally encapsulated). Dieses sind zwar alles Eigenschaften, die auch Modulen im Sinne Fodors (1983) zugeschrieben werden, zugleich entsprechen sie aber auch den Eigenschaften von Typ 1-Prozessen (mit Ausnahme der informationellen Kapselung). Daneben gibt es einige Punkte, die sich nur schwer mit der Existenz eines von erworbenem Wissen gänzlich unabhängig operierenden Moduls für wahrgenommene Kausalität vereinbaren lassen. Zum einen interpretiert Danks (2009) 
Ergebnisse von Studien, die mit Hilfe von bildgebenden Verfahren neuronale Aktivierungen während der Wahrnehmung von Kausalität aufzeichnen, als Hinweise gegen die Existenz eines distinkten neuronalen Moduls zur Wahrnehmung von Kausalität (siehe aber Blakemore et al., 2001 für eine alternative Ansicht). Darüber hinaus sind Danks (2009) zufolge bislang keine Studien bekannt, die bei Patienten mit Gehirnverletzungen selektive Ausfälle im Bereich der wahrgenommenen Kausalität nachweisen können. Des Weiteren konnten zeitlich stabile individuelle Unterschiede bezüglich der Wahrnehmung von Kausalität nachgewiesen werden. So berichten einige Versuchspersonen keine kausalen Wahrnehmungen beim klassischen launching-effect (Beasley, 1968).

Die Unveränderbarkeit des Detektors durch Lernerfahrungen ist ein zentraler Punkt der Diskussion über die Existenz eines solchen Moduls. Rips (2011) verweist auf empirische Studien, die Belege für die Ansicht liefern, dass sich zumindest einige kausale Wahrnehmungen durch Lernerfahrungen beeinflussen lassen und damit gegen das Vorliegen eines Kausalitäts-Moduls im engeren Sinne sprechen. So wurden z.B. Veränderungen der kausalen Wahrnehmung bei wiederholter Reizdarbietung berichtet (vgl. Danks, 2009). Weitere Hinweise für eine Beeinflussbarkeit kausaler Wahrnehmungen durch Erfahrungen liefern Gruber, Fink und Damm (1957), die leichte Einflüsse von vorangegangenem Training auf die kausale Beurteilung von launching-Ereignissen nachweisen konnten. Versuchspersonen, die eine Trainingsphase mit längeren zeitlichen Abständen zwischen den Bewegungen der beteiligten Objekten durchlaufen hatten, beurteilten danach eine Ereignissequenz mit zeitlichem Abstand eher kausal als eine Vergleichsgruppe, die in der Trainingsphase mit kürzeren zeitlichen Abständen konfrontiert war. Des Weiteren konnten Schlottmann und Anderson (1993) einen Einfluss individueller Prädispositionen auf Urteile zur wahrgenommenen Kausalität aufzeigen. Der Einfluss des kulturellen Hintergrundes von Versuchspersonen auf die Wahrnehmung kausaler Ereignisse (z.B. des launching effects) wurde unter anderem von Peng und Knowles (2003) untersucht. Sie konnten zeigen, dass sich chinesische und amerikanische Versuchsteilnehmer in ihren Erklärungen der Ereignisse unterschieden.

Theoretische Belege für die Beeinflussbarkeit kausaler Wahrnehmungen durch erworbenes Wissen liefern Tenenbaum und Griffiths (2003). Mit Hilfe eines auf einem dynamischen kausalen Bayes-Netz basierenden Modellierungsansatzes reinterpretieren sie ein von Anderson (1991, zit. n. Tenenbaum \& Griffiths, 2003) durchgeführtes Experiment zur kausalen Wahrnehmung. Dabei zeigen sie, dass ein Modell, welches lediglich eine reine 
bottom-up-Verarbeitung räumlicher und zeitlicher Stimuli simuliert (die angenommene Arbeitsweise eines Kausalitäts-Detektors) einem Modell, dass noch weitere Interaktionen berücksichtigt in der Erklärung des Verhaltens der Versuchspersonen unterlegen ist. Die zusätzlich berücksichtigten Interaktionen werden von den Autoren als Hinweise auf der Existenz von komplexen, aber dennoch impliziten, physikalischen Grundannahmen (i.S.v. Vorwissen) interpretiert, die den unmittelbaren kausalen Wahrnehmungen der Versuchspersonen zu Grunde liegen.

Für Vertreter des Modularitäts-Standpunktes ist die in der Literatur vorgelegte Evidenz nicht geeignet, um die Idee eines Kausalitäts-Detektors zu verwerfen (vgl. Newman, Choi, Wynn \& Scholl, 2008). So kann beispielsweise die Veränderbarkeit des Detektors mit Entwicklungsprozessen erklärt werden, die auch angeborene Module durchlaufen können. Newman und Kollegen liefern zudem experimentelle Evidenz, die ihre Auffassung eines angeborenen, aber im Laufe der Entwicklung veränderbaren Moduls für die Wahrnehmung von Kausalität stützt.

Insgesamt reicht der Umfang der empirischen Evidenz nicht aus, um die Debatte über die Existenz eines angeborenen Kausalitäts-Detektors endgültig zu entscheiden (vgl. Schlottmann, 2000). Es deutet aber einiges darauf hin, dass es zumindest einige externe Prozesse gibt, die Informationen in ein mögliches Modul einfließen lassen, das dadurch nicht mehr als informationell eingekapselt im engeren Sinne verstanden werden kann.

Unabhängig vom Ausgang dieser Debatte kann jedoch für die vorliegende Arbeit festgehalten werden, dass beide Ansätze mit der Vorstellung einer auf Typ 1-Prozessen beruhenden Wahrnehmung von Kausalität vereinbar sind. Dabei weisen die Schema-basierten Ansätze eine größere Nähe zu den in Abschnitt 2.3.1 vorgestellten Zwei-Prozess-Theorien auf, die eine Typ 1-Verarbeitung bei kausalen Phänomenen für möglich erachten. Beide Arten von Theorien gehen von einem Einfluss bestehender Gedächtnisrepräsentationen auf die Beurteilung kausaler Phänomene aus. Diese Repräsentationen werden im Zwei-ProzessAnsatz von Smith und DeCoster (2000) als Schemata verstanden, die über konkrete Lernerfahrungen aufgebaut werden. Hingegen werden die Repräsentationen in den Schemabasierten Ansätzen zur wahrgenommenen Kausalität als sehr abstrakte, domänenübergreifende kausale Schemata charakterisiert, die durch Erfahrungen aufgebaut und verändert werden oder aber auch angeboren sein können (vgl. Rips, 2011). Die Schemabasierten Ansätze gehen somit von einer Beeinflussbarkeit kausaler Wahrnehmungen durch Vorwissen in Form abstrakter kausaler Schemata aus, die durch Erfahrungen modifiziert 
werden können. In seiner Abstraktheit unterscheidet sich diese Art von Vorwissen jedoch von der Art von Vorwissen, das in den nun folgenden Abschnitten hinsichtlich seines Einflusses auf Urteile und Kategorisierungen untersucht wird.

\subsubsection{Urteilsverzerrungen}

Nachdem soeben gezeigt wurde, dass kausale Schemata möglicherweise über Typ1Prozesse die Wahrnehmung von Kausalität beeinflussen, stehen nun Urteilsprozesse im Zentrum der Betrachtung. Auch hier soll untersucht werden, ob in der Literatur Belege für einen über Typ 1-Prozesse vermittelten Einfluss kausalen Wissens bzw. der besonderen Charakteristika kausaler Beziehungen existieren. Dabei werden zunächst die Ansätze von Tversky und Kahneman (1980), sowie White (2006a) betrachtet, die unter anderem Urteilsverzerrungen untersuchen, die durch die kausale Rolle von Variablen verursacht werden. Abschließend wird auf die Studie von Luhmann et al. (2006) eingegangen, in der die Beeinflussung von Kategorisierungsurteilen durch vorhandenes Kausalwissen untersucht wird.

Im Bereich ihres „Heuristics and Biases“-Forschungsprogramm untersuchten Tversky und Kahneman (1980) auch die Rolle von Variablen in Kausalbeziehungen. Sie konnten zeigen, dass Versuchspersonen eher gewillt waren, vom Vorhandensein einer UrsacheVariablen auf das Vorhandensein einer Effekt-Variablen zu schließen als umgekehrt. Werden z.B. Urteile für die bedingten Wahrscheinlichkeiten $\mathrm{P}(\mathrm{Y} \mid \mathrm{X})$ und $\mathrm{P}(\mathrm{X} \mid \mathrm{Y})$ miteinander verglichen, so schätzt die Mehrzahl von Versuchspersonen $\mathrm{P}(\mathrm{Y} \mid \mathrm{X})$ größer ein als $\mathrm{P}(\mathrm{X} \mid \mathrm{Y})$, wenn (1) $X$ natürlicherweise als eine Ursache von $Y$ angesehen wird und (2) $P(X)=P(Y)$ ist. Da $\mathrm{P}(\mathrm{Y} \mid \mathrm{X})$ und $\mathrm{P}(\mathrm{X} \mid \mathrm{Y})$ aber aufgrund von (2) jedoch rechnerisch gleich groß sind, handelt es sich nach Tverksy und Kahneman hier um eine Urteilsverzerrung auf der Grundlage der Rollen von Variablen in einem kausalen Schema. Diese Urteilsverzerrung besteht in einer Asymmetrie zwischen prädiktiven und diagnostischen Inferenzen: Offenbar fällt es Menschen leichter, vom Vorliegen einer Ursache auf die Anwesenheit des Effektes zu schließen, als vom Vorliegen des Effektes auf die Anwesenheit der Ursache (zum Zusammenhang von prädiktivem bzw. diagnostischem Schließen und dem Charakteristikum der Gerichtetheit kausaler Beziehungen vgl. Abschnitt 2.1.4).

Des Weiteren fanden Tversky und Kahneman (1980), dass Kausalurteile unter bestimmten Bedingungen von Basisraten beeinflussbar sind. Dies geschieht, wenn die Basisraten in das dem Urteil zu Grunde liegende kausale Schema passen. Wenn nicht, so 
werden sie zu Gunsten kausal relevanter Daten vernachlässigt. Basisraten können dabei als Repräsentation von Vorwissen verstanden werden (vgl. Kahneman \& Tversky, 1973), in denen vor dem aktuellen Fall gemachte Erfahrungen in vergleichbaren Situationen zusammengefasst werden. Diese Zusammenfassung kann dabei auf zwei unterschiedliche Arten geschehen, die in statistischen bzw. kausalen Basisraten resultieren kann (Kahneman, 2011). Als statistische Basisraten werden dabei diejenigen Basisraten angesehen, die globale Informationen über die Population, aus der ein zu beurteilender Fall stammt, beinhalten. Kausale Basisraten haben dagegen eine kausale Relevanz für den zu beurteilenden Fall, indem sie in der Form von Stereotypen repräsentiert werden. Da Systeme vom Typ 1 nach Kahneman gut mit Stereotypen umgehen können, werden kausale Basisraten besser in Urteile integriert als dies bei statistischen Basisraten der Fall ist, die nur in Systemen vom Typ 2 verarbeitet werden können. Dies liegt Kahneman zufolge an der Fähigkeit von Typ 1Systemen, gut mit kausal verbundenen Informationen, wie sie beispielsweise in kausalen Schemata anzutreffen sind, umgehen zu können. Rechnerisch führen beide Arten übrigens zu identischen Ergebnissen.

Ein Schema wird von Tversky und Kahneman (1980) als ein Modell eines Systems verstanden, das dazu dient, die Outcomes des Systems vorherzusagen oder zu erklären. Die Outcomes dienen dann ihrerseits dazu, das Schema $\mathrm{zu}$ aktualisieren. Insofern können Schemata als eine Art Vorwissen verstanden werden, das die Grundlage für Urteile und Entscheidungen bildet. Schemata können unterschiedlich genau, sicher und vollständig sein. Im Alltag haben Menschen oft keine exakten Modelle der Systeme in denen sie sich bewegen. Obwohl sie sich dessen oft bewusst sind, können derlei „unsichere“ Modelle zu systematischen Urteilsverzerrungen führen. Ein bekanntes Beispiel hierfür ist die Tendenz, auf Grundlage eines bekanntermaßen zweifelhaften Modells mit großer Zuversicht Vorhersagen zu machen (over-prediction; Tversky \& Kahneman, 1980). Das unangemessen große Gewicht der aus dem zweifelhaften Modell abgeleiteten Vorhersage kann mit Hilfe der oben erwähnten kausalen Asymmetrie erklärt werden: Menschen neigen stark dazu, einen Effekt durch eine vorhandene Ursache zu erklären, auch wenn ihr Wissen über die Wirkung dieser Ursache auf den Effekt mit Unsicherheit behaftet ist. Eine weitere Konsequenz der kausalen Asymmetrie ist nach Tversky \& Kahneman (1980) der Umstand, dass es einfacher ist, neue Fakten in ein bestehendes Kausalmodell zu integrieren als das Modell auf der Grundlage der neuen Fakten zu verändern. Darüber hinaus wird ein bestehendes Modell auf der Grundlage neuer Fakten oft nur in einem unzureichenden Ausmaß verändert. Zur Illustration dienen ihnen die Ergebnisse einer Studie, in der Versuchspersonen eine auf einem 
psychologischen Test basierende Beschreibung der Persönlichkeit eines Studenten erhielten und von dieser auf seine Studienfachwahl schließen sollten. Die Beschreibung legte einen technischen Studiengang nahe. Anschließend wurde den Versuchspersonen mitgeteilt, dass die beschriebene Person tatsächlich Behindertenpädagogik studiert. Dieses Fach wurde von den Versuchspersonen zuvor als unpassend zur beschriebenen Persönlichkeit des Studenten bewertet. Im Anschluss sollten die Versuchspersonen die Beziehung zwischen Persönlichkeit und Studienfachwahl erklären. Nur wenige Versuchspersonen (21\%) stellten die Ergebnisse des Persönlichkeitstests in Frage. Die meisten erklärten die Studienfachwahl, indem sie einzelne Aspekte der beschriebenen Persönlichkeit hervorhoben oder die psychologische Bedeutung der Wahl des Studienfachs gemäß einzelner Aspekte der Persönlichkeit reinterpretierten. Die Ergebnisse der Studie legen nach Tversky und Kahneman eine Tendenz zum ,Erklären ohne Revision' (tendency to explain without revising) nahe. Ein auf zuvor erworbenem Wissen basierendes Modell bzw. Schema beeinflusst somit massiv die Interpretation neuer Informationen. Die Einfachheit mit der Menschen in der Lage sind kausal (hier: von der Ursache auf den Effekt) zu denken, inhibiert dabei nach Ansicht der Autoren die diagnostische Revision des Schemas. Das Unvermögen, die Gültigkeit des Modells der Persönlichkeit zu hinterfragen, deutet auf die Operation von Prozessen des Typ 1-Systems hin. Dieses ist nach Kahneman (2011) immer dann beteiligt, wenn die Möglichkeit einer alternativen Interpretation nicht wahrgenommen wird. Die Generierung von Erklärungen der Wahl des Studienfachs, die selbst sicherlich eher innerhalb eines Typ 2-Systems abläuft, basiert nach dieser Sichtweise auf Vorwissen, dessen Unzulänglichkeit aufgrund seiner Verarbeitung durch Typ 1-Prozesse nicht erkannt werden kann.

Die bereits von Tversky und Kahneman (1980) festgestellte Asymmetrie zwischen Ursachen und Effekten wird von White (2006a) näher untersucht. Dabei identifiziert er zwei Varianten kausaler Asymmetrie, die auftreten nachdem die Rollen der Ursache- und der Effekt-Variablen zugewiesen wurden. Die erste Variante besteht in einer Überschätzung der Bedeutung der Ursache-Variablen bei gleichzeitiger Unterschätzung oder gar Nichtbeachtung der Bedeutung der Effekt-Variablen in Bezug auf eine Veränderung der Effekt-Variable. Mit anderen Worten wird eine Änderung des Status einer Effekt-Variablen zu stark der UrsacheVariable zugeschrieben. Die zweite Variante der kausalen Asymmetrie besteht in einer Unterschätzung bzw. Nichtbeachtung der von der Effekt-Variable auf die Ursache-Variable ausgeübten Kraft. Der Ursprung dieser Variante könnte White zufolge im Vorhandensein von Vorwissen über wichtige Eigenschaften des Objektes (z.B. der zu erwartende Widerstand bei dem Versuch, es in Bewegung zu setzten) liegen. Dieses Eigenschaftswissen fließt in die 
Planung einer Manipulation des Objektes ein. Der Vorgang der Planung selbst läuft automatisch und ohne Aufmerksamkeit ab und weist damit zwei wichtige Charakteristika eines Typ 1-Prozesses auf. Somit wird das Vorwissen über die für eine Manipulation relevanten Objekteigenschaften in einem unbewussten Planungsprozess gewissermaßen verrechnet. Der unbewusste Charakter dieses Vorgangs führt nach White möglicherweise dazu, dass der Einfluss der von der Effekt-Variable (das manipulierte Objekt) auf die Ursache-Variable (das manipulierende Objekt) vernachlässigt wird oder unbeachtet bleibt. Vorwissen übt in diesem Fall somit einen über Typ 1-Prozesse vermittelten Einfluss auf ein kausales Urteil aus. Der Einfluss des manipulierten Objektes wird nur dann mittels Typ 2Prozessen verarbeitet, wenn die Objekteigenschaften nicht korrekt vorhergesehen werden können und dadurch unerwartet sind. In diesem Fall ziehen sie Aufmerksamkeit auf sich und werden bewusst verarbeitet .

Die zweite Variante der kausalen Asymmetrie erläutert White (2006a) am Beispiel des aus dem vorherigen Abschnitt bekannten launching effects: Trifft das sich bewegende Objekt A auf das stillstehende Objekt B, so überträgt sich nicht nur eine Kraft von A auf B. Gleichzeitig findet auch eine Kraftübertragung von Objekt B auf Objekt A statt, die dazu führt, dass Objekt A stehen bleibt. Nach White sehen Menschen, die keine bestimmten Erwartungen bezüglich der am launching-Ereignis beteiligten Objekte haben, in der Regel A als Ursache der Bewegung von B an. Hierbei kommen beide Varianten der kausalen Asymmetrie zum Tragen. Erstens wird der Einfluss von B auf seine Bewegung nach Kontakt mit A (die Masse von B) nicht berücksichtigt. Zweitens wird der Einfluss, den B auf A ausübt (das Stoppen von A) nicht unmittelbar wahrgenommen. White ist jedoch der Ansicht, dass Erwartungen bezüglich der Eigenschaften der beteiligten Objekte Menschen in die Lage versetzen, B als ursächlich für das Stoppen von Objekt A anzusehen. Es spricht nichts dagegen, dass diese durch Vorwissen beeinflusste kausale Wahrnehmung genauso unmittelbar auftritt wie die Wahrnehmung, dass Objekt A Objekt B anstößt. Es kommt somit darauf an, welche Erwartung man vor der Beobachtung des Ereignisses hat. Wird sie bestätigt, so reichen Typ 1-Prozesse aus, um das Ereignis zu verarbeiten und eine Interpretation zu liefern. Lediglich bei unerwarteten Ereignissen wird die Mitwirkung von Typ 2-Prozessen erforderlich.

\subsubsection{Einflüsse kausalen Vorwissens auf Kategorisierungsurteile}

In Abschnitt 2.3 wurden verschiedene Zwei-Prozess-Theorien hinsichtlich ihrer Einordnung der u.a. bei Kategorisierungsaufgaben angenommenen kognitiven 
Verarbeitungsprozesse in die Typ 1-/ Typ 2-Unterscheidung analysiert. Dabei ergab sich ein uneinheitliches Bild. Die Ansätze von Sloman (1996) sowie Strack und Deutsch (2004) gehen davon aus, dass in der Regel Typ 2-Verarbeitungsprozesse an der Bildung von Kategorisierungsurteilen beteiligt sind. Hingegen vertreten Smith und DeCoster (2000), sowie Kahneman und Frederick (2002) die Auffassung, dass Kategorisierungen auch auf der Grundlage von Typ 1-Verarbeitungsprozessen vorgenommen werden können. Im Folgenden wird eine empirische Arbeit (Luhmann et al., 2006) vorgestellt, die empirische Evidenz für die Existenz eines über Typ 1-Prozesse vermittelten Einflusses kausalen Wissens auf Kategorisierungsurteile liefert und damit mit dieser zweiten Sichtweise vereinbar ist.

Luhmann et al. (2006) gehen davon aus, dass kausales Hintergrundwissen die Gewichtung der Eigenschaften einer Kategorie beeinflusst. Die Gewichtung schlägt sich wiederum in Kategorisierungsurteilen nieder. Dies ist auch dann der Fall, wenn eine Kategorisierung unter Bedingungen vorgenommen wird, die Typ 2-Verarbeitungsprozesse verhindern. Diese Ansicht widersprechen damit denjenigen Ansätzen in der Literatur, die für eine Berücksichtigung (kausalen) Hintergrundwissens langsame, deliberative (Typ 2-) Verarbeitungsprozesse als notwendig erachten. Die Autoren gehen jedoch nicht davon aus, dass kausales Wissen den Urteilsprozess selbst beeinflusst. Sie sind vielmehr der Ansicht, dass während des Erlernens kausaler Strukturen Eigenschaftsgewichte extrahiert und im Gedächtnis gespeichert werden. Der Urteilsprozess basiert damit lediglich aus einem ähnlichkeitsbasierten Abgleich der präsentierten Eigenschaftsausprägungen mit den vorgespeicherten Eigenschaftsgewichten. Die Grundlage für diese Annahmen bilden die Ergebnisse von Experimenten, in denen Luhmann und Kollegen Belege dafür erbrachten, dass Versuchspersonen bei Kategorisierungsentscheidungen unter Zeitdruck den kausalen Statuseffekt (causal status effect, Ahn, Kim, Lassaline \& Dennis, 2000) zeigen. Dieser Effekt besagt, dass die Position einer Variablen in einer kausalen Struktur einen Einfluss auf die Gewichtung dieser Variablen bei Kategorisierungsurteilen ausübt. Da im empirischen Teil der vorliegenden Arbeit ebenfalls kausale Kategorisierungsaufgaben untersucht werden, bei denen Vorhersagen für Urteile auf Grundlage des kausalen Status von Variablen abgeleitet werden, soll der Effekt an dieser Stelle näher erläutert werden. Im Anschluss werden die wesentlichen Aspekte der von Luhmann et al. (2006) durchgeführten Experimente vorgestellt.

Der kausale Status einer Variablen ist umso höher, je mehr andere Variablen von dieser kausal abhängig sind und spiegelt die Bedeutung der Variable innerhalb des Systems wieder. Dem kausalen Statuseffekt liegt somit die Annahme zu Grunde, dass Menschen 
Ursache-Variablen in Bezug auf Kategorisierungen als wichtiger und essenzieller ansehen als Effekt-Variablen. Dieses Phänomen lässt sich an folgendem einfachen Beispiel erklären (vgl. Rehder \& Kim, 2010): Gegeben sei eine kausale Kette mit den Variablen A, B und C. Dabei wird B von A und C von B verursacht. Somit ist A die in der kausalen Hierarchie am höchsten rangierende Variable und besitzt damit auch den höchsten kausalen Status. B, das ja sowohl ein Effekt (von A) als auch eine Ursache (von C) ist, hat die zweithöchste Position in der Kausalstruktur und somit auch den zweithöchsten kausalen Status. C, das ausschließlich ein Effekt (von B) ist, hat den niedrigsten kausalen Status in der Kette. Nimmt man nun an, dass die Variablen A, B und C jeweils die beiden Ausprägungen ,anwesend“ (1) oder „abwesend“(0) annehmen können und dass beim Prototyp eines Kategorienmitgliedes alle drei Variablen die Ausprägung ,,anwesend“ aufweisen, so ergibt sich aufgrund des kausalen Status der Variablen folgendes Bild: Die Wahrscheinlichkeit einer Zuordnung zur Kategorie ist für die Variablenkombination $(\mathrm{A}=1, \mathrm{~B}=1, \mathrm{C}=1)$ am höchsten und für $(\mathrm{A}=0, \mathrm{~B}=0, \mathrm{C}=0)$ am geringsten. Aufgrund des unterschiedlichen kausalen Status der einzelnen Variablen hat die Kombination $(\mathrm{A}=1, \mathrm{~B}=1, \mathrm{C}=0)$ eine höhere Wahrscheinlichkeit der Zuordnung zur Kategorie als $(A=0, B=1, C=1)$, obwohl in beiden Fällen jeweils zwei Variablen anwesend und eine Variable abwesend ist.

Wichtigste Voraussetzung für das Auftreten des kausalen Statuseffektes ist vorhandenes Wissen über die Struktur des Kausalmodells. Dieses Wissen wurde den Versuchspersonen in den Experimenten von Luhmann et al. (2006) für mehrere Kategorien explizit vermittelt (z.B: „A verursacht B, B verursacht C“, wobei A, B und C für die Eigenschaften einer zu erlernenden Kategorie stehen). Zudem wurden die kausalen Relationen graphisch dargestellt. Nach der Präsentation einer Kategorie wurden die Versuchspersonen darüber hinaus aufgefordert, Begründungen für die instruierten Kausalrelationen aufzuschreiben. Die Versuchspersonen sollten sich so intensiv mit der jeweiligen Kausalstruktur auseinandersetzen. In einer weiteren Phase wurde sichergestellt, dass die Versuchspersonen in der Lage waren, den kausalen Status der Eigenschaften der gelernten Kategorien zuverlässig zu bestimmen. Danach wurde die Zeit eingeschränkt, die für diese Bestimmung zu Verfügung stand. Dies sollte die Anwendung des zuvor erlernten Kausalwissens automatisieren. In einer anschließenden Testphase wurden den Versuchspersonen Exemplare der verschiedenen Kategorien präsentiert, bei denen jeweils eine Eigenschaft abwesend war. Die Aufgabe bestand darin, die Wahrscheinlichkeit der Kategorienzugehörigkeit für die verschiedenen Exemplare zu beurteilen. In einer Hälfte der Durchgänge wurden die Versuchspersonen aufgefordert, so schnell wie möglich zu antworten. 
In der anderen Hälfte der Durchgänge sollten sie sich so viel Zeit wie nötig nehmen. Obwohl sich die Zeiten in den beiden Arten von Durchgängen deutlich unterschieden (die Reaktionszeiten in den Durchgängen mit Aufforderung zum schnellen Antworten waren im Durchschnitt halb so groß wie in den anderen Durchgängen) beurteilten die Versuchspersonen in beiden Arten von Durchgängen die Wahrscheinlichkeit der Zugehörigkeit zu einer Kategorie tendenziell in Abhängigkeit vom kausalen Status der nicht vorhandenen Eigenschaft. Es zeigte sich somit unter beiden Instruktionen ein kausaler Statuseffekt.

In einem weiteren Experiment wurde unter anderem die den Versuchspersonen für die Beurteilung der Kategorienzugehörigkeit zur Verfügung stehende Zeit begrenzt. Auch in diesem Experiment zeigte sich die Tendenz, dass Versuchspersonen Exemplare, bei denen die fehlende Eigenschaft einen niedrigen kausalen Status besitzt, als eher der Kategorie zugehörig beurteilen als Exemplare, bei denen die fehlende Eigenschaft einen hohen kausalen Status aufweist. Dieser Effekt macht sich bei einer Präsentationsdauer der Exemplare von $500 \mathrm{~ms}$ oder mehr bemerkbar.

Wie bereits erwähnt gehen Luhmann et al. (2006) davon aus, dass während der Urteilsbildung ähnlichkeitsbasierte Prozesse ablaufen. Allerdings können die berichteten Befunde auch durch eine kausale Informationsverarbeitung während der Urteilsfindung, die auf Typ 1-Prozessen basiert, erklärt werden. So könnte eine intuitive kausale Urteilsbildung beispielsweise auf Grundlage holistischer Typ 1-Verarbeitungsprozesse (z.B. ein Musterabgleich unter Berücksichtigung kausaler Einflussfaktoren) ablaufen, wie dies im Zwei-System-Ansatz von Kahneman und Frederick (2002) nahegelegt wird. Insgesamt liefern die von Luhmann et al. (2006) vorgestellten Studien unabhängig von der Interpretation ihrer Ergebnisse Belege für die Möglichkeit des Wirkens schneller, unbewusster Typ 1Prozesse bei Kategorisierungsurteilen auf der Grundlage kausalen Vorwissens.

Die in diesem Abschnitt vorgestellten Ansätze liefern einige Hinweise auf die Möglichkeit der Existenz intuitiver Verarbeitungsprozesse, die unter Berücksichtigung kausaler Informationen in einem Typ 1-System ablaufen. Neben dem Phänomen der wahrgenommenen Kausalität, das eine große Nähe zu perzeptuellen Prozessen aufweist, scheinen über Typ 1-Prozesse vermittelte kausale Einflüsse auch bei kognitiven Prozessen höherer Ordnung wie den von Tversky und Kahneman (1980) untersuchten Urteilsprozessen oder den Kategorisierungsurteilen, die den Gegenstand der Studie von Luhmann et al. (2006) bildeten, eine wichtige Rolle zu spielen. Im folgenden Abschnitt sollen nun kausale Ansätze 
untersucht werden, die ein Wirken von Typ 2-Prozessen bei Urteilen und Entscheidungen nahelegen.

\subsubsection{Theorien der kausalen Deliberation}

\subsubsection{Logische Theorien kausaler Deliberation}

Eine frühe Untersuchung des Einflusses kausalen Vorwissens auf logisches Schlussfolgern liefern Cheng und Holyoak (1985). Sie beschäftigen sich mit den Fehlern, die Menschen beim logischen Schließen begehen und erklären sie mit der Kongruenz des Schlusses zu aktivierten pragmatic reasoning schemas, die bestimmte Schlüsse nahelegen. Korrespondiert ein solches Schema mit einer logischen Schlussfolgerung, so ist die Wahrscheinlichkeit des Auftretens logischer Fehler geringer als dies bei einer Inkongruenz des Schlusses mit dem Schema der Fall wäre. Pragmatice reasoning schemas werden als abstrakte Wissenstrukturen verstanden, die auf Lebenserfahrungen (z.B. über die Verursachung von Ereignissen) basieren. Cheng und Holyoak beschreiben sie als Sets von generalisierten, kontext-sensitiven Regeln, die dazu dienen nicht-logische (z.B. Ursache oder Vorhersage) und formallogische Begriffe (z.B. „wenn-dann“ oder „nur dann“) zu interpretieren. Im Prozess des logischen Schließens werden die pragmatic reasoning schemas mittels Vorwissen induziert und evoziert.

Eine Abweichung eines Schlusses von seiner formallogisch korrekten Lösung kann nach Cheng und Holyoak (1985) beispielsweise beim diagnostischen Schließen vorliegen. In einem kausalen Schema kann es sinnvoll sein, vom Vorliegen des Effektes auf das Vorliegen der Ursache zu schließen, wenn lediglich eine Ursache als für den Effekt verantwortlich wahrgenommen wird. Ein solcher Schluss führt formallogisch hingegen zum Fehlschluss der Affirmation der Konsequenz. Darüber hinaus kann die Aktivierung kausaler Schemata die Bewertung kontrapositive Transformationen (der Überführung einer Aussage der Form „Wenn A, dann B“ in die Form „Wenn nicht-B, dann nicht-A“) beeinflussen. Hierbei spielt die im kausalen Schema repräsentierte zeitliche Abfolge von Ursache und Effekt eine entscheidende Rolle. Treten Ursache und Effekt gleichzeitig auf, so wird die kontrapositive Transformation als sinnvoll angesehen, wie dies von den Autoren am Beispiel der kontrapositiven Transformation der Aussage „Wenn Rauch da ist, dann ist da ein Feuer“ in die Aussage „Wenn kein Feuer da ist, dann ist da kein Rauch“ gezeigt wird. Ist im kausalen Schema hingegen eine eindeutige zeitliche Abfolge repräsentiert, so wird die kontrapositive Transformation nicht als sinnvoll angesehen. Als Beispiel dient den Autoren hier die 
Transformation der Aussage „Wenn die Bombe explodiert, dann werden alle sterben“ in die Aussage „Wenn nicht alle sterben, dann wird die Bombe nicht explodieren“.

Obwohl Cheng und Holyoak (1985) keine konkreten Aussagen über die Natur der kognitiven Prozesse machen, die bei der Beeinflussung logischer Schlussfolgerungen durch pragmatic reasoning schemas ablaufen, gibt es einige Hinweise auf die Beteiligung von Typ 2-Prozessen: Die Schemata sind als Ansammlungen von generalisierten, kontext-sensitiven Regeln definiert, die abstrakte Wissensstrukturen abbilden. Die Verarbeitung abstrakter Repräsentationen und die Anwendung von Regeln werden in der Zwei-System-Debatte den Typ 2-Systemen zugeordnet (vgl. Evans, 2008; Abschnitt 2.2.2). Cheng und Holyoak (1985) gehen ferner davon aus, dass Schemata auf der Basis von Vorwissen evoziert werden können. Geht man davon aus, dass das Ergebnis einer Evokation bewusst ist, kann auch hier von einem Vorgang im Typ 2-System ausgegangen werden. Überdies werden die Versuchspersonen in den berichteten Experimenten ausdrücklich aufgefordert, gründlich über ihre Entscheidung nachzudenken, sich so viel Zeit wie nötig zu lassen, Erklärungen für ihre Antworten aufzuschreiben und ihre Antworten gegebenenfalls zu korrigieren. Diese Bedingungen können als Aufforderung zum bewussten (Typ 2-) Denken verstanden werden. Insgesamt liegt somit der Schluss nahe, dass Cheng und Holyoak von einer Beteiligung von Typ 2-Prozessen beim Einfluss der pragmatic reasoning schemas auf logische Schlussfolgerungen ausgehen. Da diese Schemata auch kausaler Natur sein können, ist der vorgestellte Ansatz als eine Theorie kausaler Deliberation zu verstehen.

In ähnlicher Weise kann auch die Studie von Cummins et al. (1991) interpretiert werden, die den Einfluss von Vorwissen auf die Beurteilung kausaler Szenarien, die mittels kausaler Konditionale (Modus ponens, Modus tollens, Negation des Antezedens, Affirmation der Konsequenz) beschrieben werden, zum Gegenstand hat. Dabei wurde die Bewertung von auf den Konditionalen basierenden Konklusionen in Abhängigkeit von Vorwissen über alternative Erklärungen für die kausalen Szenarien untersucht. Die Akzeptanz der Konklusionen für Modus ponens und Modus tollens wurde dabei vor allem durch die Anzahl der bekannten Möglichkeiten der Verhinderung des Auftretens des Effektes bei Anwesenheit seiner Ursache (disabling conditions) beeinflusst. Hingegen war die Akzeptanz der Konklusionen bei einer Negation des Antezedens oder einer Affirmation der Konsequenz von der Anzahl alternativer Ursachen abhängig. Cummins und Kollegen machen ähnlich wie Cheng und Holyoak (1985) keine expliziten Angaben über die kognitiven Prozesse, die dem Einfluss des kausalen Wissens zu Grunde liegen. Sie gehen jedoch davon aus, dass der 
Stimulus (hier: das kausale Konditional) zu einem Abruf alternativer Erklärungen (hier: Ursachen oder disabling conditions) führt, die aus erinnerten oder konstruierten Gedächtnisrepräsentationen bestehen. Diese Repräsentationen werden dann zur Interpretation des vorliegenden Stimulus herangezogen. Es liegt nahe, diesen Prozess in einem Typ 2System zu verorten, zumal von einer durch den Stimulus ausgelösten Suche nach Alternativen ausgegangen wird, die als eine domänenübergreifende Denkstrategie bei der Bearbeitung kognitiver Aufgaben charakterisiert werden kann (Cummins et al., 1991).

Die bereits im Abschnitt 2.1 kurz vorgestellte Mental Model Theory of Causal Meaning and Reasoning (Goldvarg \& Johnson-Laird, 2001) stellt einen weiteren Ansatz dar, der kausale Schlüsse als abhängig von Typ 2-Prozessen erscheinen lässt. Die Kernaussage der Theorie ist, dass Menschen kausale Beziehungen in Form von auf Propositionen basierenden mentalen Modellen repräsentieren und kausale Schlüsse auf der Grundlage ebendieser Modelle ziehen und bewerten. Ein mentales Modell umfasst dabei jeweils eine wahre Möglichkeit der Realisation der Prämissen einer kausalen Aussage und stellt den Endzustand einer Wahrnehmung, einer Vorstellung oder des Verständnisses eines Diskurses dar. Struktur und Inhalt eines mentalen Modells erfassen die Gemeinsamkeiten der verschiedenen Formen, in denen die abgebildete wahre Möglichkeit der Realisation der Prämissen einer kausalen Aussage auftreten kann. Dabei wird auch die zeitliche Priorität der Ursache vor ihrem Effekt berücksichtigt. Die kausale Aussage „A wird B verursachen“ wird nach Goldvarg und Johnson-Laird (2001) beispielsweise mittels dreier mentaler Modelle repräsentiert, die jeweils eine Möglichkeit umfassen: „A tritt auf, B tritt auf“, „A tritt nicht auf, B tritt auf“ und „A tritt nicht auf, B tritt nicht auf".

Der Theorie von Goldvarg und Johnson-Laird (2001) zufolge wird dabei jedoch vor allem das Modell „A tritt auf, B tritt auf“ explizit repräsentiert. Die beiden übrigen Modelle, in denen der Antezedens (A) nicht zutrifft, werden implizit in Form einer sog. mentalen Fußnote (mental footnote) abgelegt. Diese mentale Fußnote kann in einem Vorgang der Konkretisierung (fleshing out), der jedoch mit zusätzlichen kognitiven Anstrengungen verbunden ist, dazu genutzt werden, die mit ihr verbundenen mentalen Modelle ins Arbeitsgedächtnis zu rufen. In diesem Fall werden alle wahren Möglichkeiten der Realisation einer Aussage mittels vollständig expliziten Modellen (fully explicit models) repräsentiert. Allerdings ist es nach Ansicht der Autoren für Menschen nicht einfach, alle vollständig expliziten Modelle gleichzeitig aktiv im Arbeitsgedächtnis zu halten (,Three possibilities are hard to hold in mind at the same time“, Goldvarg \& Johnson-Laird, 2001, S. 573). Um diese 
Beanspruchung des Arbeitsgedächtnisses zu minimieren beschränken sich Menschen darauf, sich auf für bestimmte Kausalrelationen besonders repräsentative Möglichkeiten zu fokussieren und die übrigen Modelle in Form mentaler Fußnoten abzulegen.

Auf Grundlage dieser Darstellung erscheint es gerechtfertigt, die Mental Model Theory of Causal Meaning and Reasoning (Goldvarg \& Johnson-Laird, 2001) zu der Gruppe von Theorien zu zählen, die von einer Beteiligung von Typ 2-Verarbeitungsprozessen bei der Urteilsbildung annehmen. Bei den im Rahmen der Theorie betrachteten kausalen Schlüssen legen insbesondere die Beteiligung des Arbeitsgedächtnisses bei der expliziten Repräsentation einzelner oder aller mentaler Modelle einer Aussage sowie der Vorgang der Konkretisierung eine Beteiligung von Typ 2-Prozessen nahe.

\subsubsection{Die Rolle mentaler Simulationen}

Weitere Belege für die Verarbeitung kausaler Informationen mittels Typ 2-Prozessen liefern Sloman und Lagnado (2005). In ihren Experimenten konnten sie zeigen, dass Menschen in ihren Schlüssen sensitiv für die Auswirkungen von Interventionen in ein Kausalmodell sind. Nach der Theorie der kausalen Bayes-Netze (z.B. Pearl, 2009) wird bei einer Intervention eine Variable auf einen bestimmten Wert gesetzt und die Verbindung zu ihrer/ihren eigentlichen Ursache(n) gekappt (graph surgery, vgl. Abschnitt 2.1.5). Nach einer Intervention kann nicht mehr auf die normalen Ursachen der Variablen, auf die interveniert wurde, geschlossen werden. Ein Schluss auf ihre(n) Effekt(e) ist jedoch weiterhin möglich. Dadurch unterscheiden sich Interventionen von reinen Beobachtungen, da bei letzteren sowohl Schlüsse auf die Ursache(n) einer Variablen als auch auf ihre Effekte möglich sind. Sloman und Lagnado (2005) untersuchten in 6 Experimenten, ob Versuchspersonen sensitiv für die Folgen von Interventionen sind. Dabei zeigte sich, dass Menschen die hinter den Interventionen stehende Logik verstehen und sie für ihre Schlussfolgerungen anwenden, wenn diese auf kausalen Szenarien basieren. So konnten die Experimente zeigen, dass sich in kausalen Szenarien Schlüsse auf Grundlage von Interventionen von Schlüssen auf Grundlage von Beobachtungen unterschieden. In nicht-kausalen Szenarien wurde die Interventionslogik hingegen nicht angewandt.

Die Berücksichtigung der Auswirkungen von Interventionen erfordert eine Reihe kognitiver Vorgänge, die in der Zwei-System-Debatte als Typ 2-Prozesse charakterisiert werden: Die Modifikation eines Kausalmodells aufgrund der Information über das Vorliegen einer Intervention und die Vorhersage der Folgen dieser Intervention mittels mentaler 
Simulation ist kaum ohne Aufmerksamkeit, Bewusstheit und Zeit vorstellbar. Außerdem können diese Prozesse als regelbasiert verstanden werden, da die Charakteristika kausaler Beziehungen berücksichtigt werden müssen.

Eine weitergehende Anlayse der Rolle mentaler Simulationen beim kausalen Schließen liefert Lagnado (2011). Dabei diskutiert er die Möglichkeit, dass mentale Simulationen diejenigen Prozesse darstellen, die dem kausalen Schließen zu Grunde liegen. Der Kerngedanke hierbei ist, dass Menschen mentale Modelle von Objekten, Ereignissen oder Tatbeständen bilden und für mentale Simulationen nutzen, um zu Schlussfolgerungen zu gelangen. Aufbauend auf Craik (1952) wird angenommen, dass Menschen die Auswirkungen ihrer Handlungen in einem mentalen Modell der externen Welt simulieren. Das hierbei verwendete mentale Modell der Welt beinhaltet die für die in Frage kommenden Handlungen relevanten kausalen Mechanismen wie sie auch in der realen Welt vorzufinden sind. Lagnado (2011) erweitert die Ideen Craik's (1952) um vier Prinzipien, die er aus der Forschung zum mechanischen Schließen (Hegarty, 2004) entleiht. Erstens werden Simulationen lediglich in einem Ausschnitt des Gesamtmodells ausgeführt, der die relevanten Kausalverbindungen enthält. Zweitens beruhen Simulationen nicht ausschließlich auf der Manipulation visueller Vorstellungen, sondern können auch abstraktere Komponenten beinhalten. Drittens können auch motorische Repräsentationen Bestandteil mentaler Simulationen sein. Viertens stellen mentale Simulationen nur eine unter mehreren möglichen Strategien zum Lösen von Inferenzproblemen dar, mit denen sie auch kombiniert werden können. Auch wenn Lagnado (2011) nicht ausdrücklich von Typ 2-Prozessen spricht, ist doch davon auszugehen, dass die von ihm beschriebenen Vorgänge nicht gänzlich ohne sie auskommen.

Die Vorteile eines Simulations-basierten Ansatzes kausaler Inferenz liegen Lagnado (2011) zufolge zum einen in der Erklärung der Ergebnisse empirischer Studien, die zeigen konnten, dass sich die Inferenzleistung von Probanden verbesserte, wenn konkretes Stimulusmaterial benutzt und räumliche Imagination erleichtert wurde. Zudem kann der Ansatz Situationen erklären, in denen Menschen fehlerhafte Inferenzen lieferten. Zum anderen kann mit dem Simluations-basierten Ansatz erklärt werden, warum es Menschen leichter fällt prädiktive als diagnostische Inferenzen durchzuführen: Während im ersten Fall eine relativ einfache, vorwärtsgerichtete Simulation (von der Ursache zum Effekt) ausreicht, ist im zweiten Fall eine komplexere Simulation nötig, da berücksichtigt werden muss, welche anderen potentiellen Ursachen den Effekt ebenfalls hätten hervorrufen können. 


\subsubsection{Kausale Entscheidungstheorien}

Mentale Simulationen von Interventionen bilden den Kern einer kausalen Entscheidungstheorie, die Sloman und Hagmayer (2006; Hagmayer \& Sloman, 2009) mit der Causal Model Theory of Choice vorstellen. Die nun folgende ausführliche Darstellung dieser Theorie ist der Tatsache geschuldet, dass sie die einzige dem Autor der vorliegenden Arbeit bekannte kausale Entscheidungstheorie ist, die sich direkt in die 2-System-Debatte einordnen lässt. Sie gehen davon aus, dass Menschen vereinfachte Kausalmodelle einer Entscheidungssituation nutzen, um mit Hilfe simulierter Interventionen in diese Modelle die für sie beste Handlungsoption auszuwählen. Es wird angenommen, dass Entscheider ihre Entscheidungen für bestimmte Handlungen als Interventionen in ein kausales System verstehen, durch die alle kausalen Beziehungen zwischen der Zielvariable der Intervention und ihren normalen Ursachen aufgelöst werden. Dieser Prozess des undoing (vgl. Sloman, 2005) unterscheidet das Kausalmodell eines Entscheiders von dem eines unbeteiligten Beobachters: Der Beobachter repräsentiert die Entscheidung als in das Modell eingebetteten Knoten, der von anderen Variablen wie z.B. dem Entscheidungsprozess und situativen Einflüssen bestimmt wird. Dagegen werden im Modell des Entscheiders die kausalen Einflüsse auf die Entscheidung entfernt, da die Entscheidung als willentliche Festsetzung der Entscheidungs-Variablen auf einen bestimmten Wert durch den Entscheider angesehen wird. Aus diesem Verständnis einer Entscheidung als Intervention folgt, dass durch die Intervention keine neuen Informationen über die Ursachen der Entscheidung gewonnen werden können. Darüber hinaus gibt der willentliche Charakter der Entscheidung für den Entscheider einen deutlichen Hinweis auf die Beteiligung von Typ 2-Prozessen.

Der Causal Model Theory of Choice (Sloman und Hagmayer, 2006; Hagmayer \& Sloman, 2009) zufolge kann der Entscheidungsprozess in drei Phasen eingeteilt werden. In der ersten Phase wird in vier Schritten ein kausales Modell der Entscheidungssituation konstruiert. Erstens werden die Ziele der entscheidenden Person in Form einer Verteilung präferierter kausaler Konsequenzen (distribution of prefered causal consequences) repräsentiert. Zweitens werden Faktoren identifiziert, die mit diesen Konsequenzen in einer kausalen Beziehung stehen. Drittens wird ein kausales Modell konstruiert, das beschreibt wie die Faktoren die kausalen Konsequenzen erzeugen. Viertens werden auf der Grundlage der verfügbaren Informationen geschätzt, wie wahrscheinlich die präferierten Konsequenzen unter bestimmten Bedingungen eintreten. Außerdem wird in diesem Schritt geprüft, ob es unbeobachtete Variablen gibt, die aufgrund der verfügbaren Informationen als anwesend 
angenommen werden müssen. Das Ergebnis dieser Phase wird von Sloman und Hagmayer (2006; Hagmayer \& Sloman, 2009) als das „Weltmodell“ (world model) bezeichnet, da es sich um ein Kausalmodell handelt, das ein kausales System in der Welt repräsentiert. Das so konstruierte kausale Modell der Entscheidungssituation dient in der zweiten Phase als Basis für die Repräsentation der zur Verfügung stehenden Handlungsoptionen. Dabei wird davon ausgegangen, dass die Wahl einer bestimmten Handlung als (exogene) Intervention in das Kausalsystem repräsentiert wird. Das Weltmodell wird in dieser Phase wie folgt in ein „Entscheidungsmodell“ (choice model) transformiert: Die Interventionsmöglichkeit wird als Knoten im Kausalmodell repräsentiert, der ausschließlich mit der Zielvariable der Intervention verbunden ist. Des Weiteren wird die Verbindung zwischen der Zielvariable und ihren eigentlichen Ursachen gekappt. Zuletzt werden über Simulationen der kausalen Konsequenzen der Handlungsoption(en) die aus der Intervention resultierenden Wahrscheinlichkeiten des Eintretens der gewünschten Konsequenzen (interventional probabilities) abgeleitet. In der dritten Phase wird überprüft, ob eine Handlungsoption die Wahrscheinlichkeit des Auftretens des gewünschten Effektes erhöht. Eine mögliche Handlung wird nur dann weiter als Option betrachtet, wenn sie die Wahrscheinlichkeit des Eintretens der präferierten Konsequenzen erhöht. Ist dies der Fall und steht nur eine mögliche Handlung zur Auswahl, so wird diese bei Vorliegen eines positiven Kosten-Nutzen-Verhältnisses gewählt. Ein positives Kosten-Nutzen-Verhältnis liegt dann vor, wenn die Kosten der Handlung geringer als der durch sie verursachte zusätzliche Nutzen durch die Erhöhung der Wahrscheinlichkeit des Eintretens der gewünschten Konsequenzen sind. Hierbei ist der Theorie zufolge eine qualitative Abschätzung ausreichend. Liegen hingegen mehrere Handlungsmöglichkeiten vor, so werden kausale Erwartungswerte für die einzelnen Optionen gebildet und verglichen, die auf den interventional probabilities basieren. Es muss für jede Option ein eigenes Entscheidungsmodell konstruiert werden, um die entsprechenden kausalen Erwartungswerte ableiten zu können. Die Wahrscheinlichkeit der Wahl einer Handlungsoption wird schließlich als proportional zu ihrem kausalen erwarteten Nutzen angenommen. Im Anschluss an die Entscheidung können Lernprozesse auftreten durch die das Kausalmodell der Entscheidungssituation aktualisiert wird. Hagmayer und Sloman (2009; siehe auch Nichols \& Danks, 2007; Robinson, Sloman, Hagmayer \& Hertzog, 2010; Saito \& Shimazaki, 2011 für weitere empirische Prüfungen der Theorie) liefern in vier Experimenten Evidenz für die zentralen Annahmen der Theorie.

Im Fokus der Causal Model Theory of Choice (Sloman und Hagmayer, 2006; Hagmayer \& Sloman, 2009) stehen Entscheidungen auf der Basis von Deliberationen. Die 
Evaluation der verschiedenen Handlungsoptionen erfolgt im Rahmen eines mentalen Simulationsprozesses, der bewusstes Nachdenken über die aufgrund des verwendeten Kausalmodells möglichen Konsequenzen der Entscheidungsalternativen erfordert. Dies geschieht unabhängig davon, ob die Handlungen im Kausalmodell normalerweise überlegt oder reflexartig getroffen werden (Hagmayer \& Sloman, 2009, Experiment 3). Eine für die vorliegende Arbeit interessante Frage ist, ob sich aus dem Fokus auf deliberative Entscheidungen die Annahme ableiten lässt, dass gemäß der Causal Model Theory of Choice kausale Entscheidungen immer nach bewusstem Nachdenken getroffen werden. Insbesondere im Hinblick auf die Simulation der kausalen Konsequenzen möglicher Interventionen erscheint diese Annahme als plausibel.

Des Weiteren stellt sich die Frage, wie Entscheidungen auf der Grundlage zuvor bereits gelernter Kausalmodelle getroffen werden. Prinzipiell sollte die Theorie auch hier anwendbar sein, da durch ein bereits vorhandenes Kausalmodell der Entscheidungssituation lediglich die erste Phase der Konstruktion eines Weltmodells beeinflusst wäre. Bewertet ein Entscheider das vorhandene Kausalmodell als der Situation angemessen, so sollte die Modifikation zum Entscheidungsmodell und die anschließende Auswahl der Handlungsoption auf der Grundlage des Ergebnisses der mentalen Simulation der verschiedenen Optionen analog zu den weiter oben beschriebenen Phasen ablaufen. Auch in diesem Fall wäre also von einer Beteiligung von Typ 2-Prozessen am Entscheidungsvorgang auszugehen. Eine Ausnahme stellen Interventionsentscheidungen dar, die in der Vergangenheit bereits so häufig getroffen wurden, dass sie bei Vorliegen bestimmter Rahmenbedingungen automatisch ablaufen. Hierbei handelt es sich dann aber nicht um eine Entscheidung im Sinne der Theorie, sondern um eine Entscheidung auf der Grundlage einer assoziativen Verknüpfung. Hagmayer \& Sloman (2009) gehen nicht davon aus, dass kausales Entscheiden immer dann stattfindet, wenn es möglich ist. Sie nehmen vielmehr an, dass kausales Entscheiden vor allem bei neuartigen Aufgaben angewendet wird, bei denen ein gewisses Maß an (kausalem) Hintergrundwissen besteht. Zudem müsse die Aufgabe wichtig genug sein, um den für die Entscheidungsfindung nötigen Aufwand zu rechtfertigen.

Insgesamt geht die Causal Model Theory of Choice (Sloman \& Hagmayer, 2006; Hagmayer \& Sloman, 2009) eindeutig davon aus, dass für die von ihr behandelten Interventionsentscheidungen eine Beteiligung von Typ 2-Prozessen notwendig ist. Dadurch unterscheidet sie sich von den anderen in diesem Abschnitt vorgestellten Ansätzen, deren Einordnung in die 2-System-Debatte auf der Grundlage von Interpretationen der durch sie 
(mehr oder weniger expliziten) vorgenommenen Annahmen bezüglich der zu Grunde liegenden kognitiven Prozesse durchgeführt wurde.

Ein aus der Causal Model Theory of Choice (Sloman \& Hagmayer, 2006; Hagmayer \& Sloman, 2009) abgeleiteter Ansatz ist die Intervention-Finder-Heuristic (Meder et al., 2010). Die Heuristik besagt, dass Menschen beim Entscheiden über die Wahl einer Intervention in ein ihnen unbekanntes kausales Modell, die einen gegebenen Effekt mit der größten Wahrscheinlichkeit hervorruft folgende Schritte durchführen: Zunächst wird mittels der beobachteten Variablen eine Art kausales „Skelett“ (,skeletal“ causal model) gebildet, das dazu dient, die potenziellen Ursachen des interessierenden Effektes zu identifizieren. Das Modell wird als Skelett bezeichnet, da es weder die genaue Kausalstruktur noch deren Parameter erfasst. Die beobachteten Variablen werden lediglich in Bezug auf den interessierenden Effekt auf ihren Status als mögliche direkte oder indirekte Ursache hin bewertet. Dabei dienen die bereits beschriebenen Charakteristika kausaler Beziehungen als Hinweise auf die kausalen Rollen der einzelnen Variablen. Somit wird die Anzahl an möglichen Interventionspunkten durch die Berücksichtigung des Merkmals der Gerichtetheit kausaler Beziehungen eingeschränkt. Mittels der bedingten Wahrscheinlichkeit $\mathrm{P}($ Effekt|mögliche Ursache) wird anschließend diejenige Variable bestimmt, deren Aktivierung mit der größten Wahrscheinlichkeit zum Hervorrufen des Effektes führt. Im Gegensatz zur Causal Model Theory of Choice (Sloman \& Hagmayer, 2006; Hagmayer \& Sloman, 2009) benötigt die Intervention-Finder Heuristic (Meder et al., 2010) weder das vollständige Kausalmodell noch die Berechnung der Interventions-Wahrscheinlichkeiten (interventional probabilities) um zu einer Entscheidung zu gelangen. Dennoch ist davon auszugehen, dass auch in diesem Ansatz die Wahl der besten Intervention unter Beteiligung von Typ 2-Prozessen abläuft, da die einzelnen Schritte der Heuristik die aktive Erzeugung mentaler Repräsentationen sowie deren Nutzung zur Simulation der Auswirkung der verschiedenen Handlungsalternativen erfordern.

Die in diesem Abschnitt vorgestellten kausalen Ansätze legen die Beteiligung von Typ 2-Prozessen an der Urteils- und Entscheidungsfindung nahe, auch wenn keine der vorgestellten Theorien diese Einordnung explizit vornimmt. Die in den verschiedenen Ansätzen untersuchten Phänomene unterscheiden sich vor allem durch ihre höhere Komplexität zu den im Abschnitt zur kausalen Intuition vorgestellten Ansätzen. Darüber hinaus beschäftigen sie sich mit Aufgaben, die klassischerweise mit bewussten und gründlichen Nachdenken assoziiert sind wie z.B. die Aufgaben zum logischen Schließen im 
Ansatz von Cheng und Holyoak (1985). Der Prozess der mentalen Simulation nimmt eine wichtige Stellung in einigen der vorgestellten Ansätzen (Meder et al., 2010, Sloman \& Hagmayer ,2006); Sloman \& Lagnado,2005) ein und kann als Prozess vom Typ 2 verstanden werden. Daher sind die in diesem Abschnitt vorgestellten Ansätze den Systemen vom Typ 2 zuzuordnen. Dies bedeutet jedoch nicht, dass ausschließlich Typ 2-Prozesse an den Urteilen und Entscheidungen beteiligt sind. Vielmehr wird hier der Sichtweise von Evans (2011; vgl. Abschnitt 2.2.2) gefolgt, die davon ausgeht, dass auch Typ 1-Prozesse an der Operation von Typ 2-Systemen beteiligt sind.

\subsubsection{Theorien der kausalen Intuition und Deliberation}

In den beiden vorherigen Abschnitten ging es um kausale Theorien, die entweder kausale Phänomene zu erklären versuchten, die mit Verarbeitungsprozessen vom Typ 1 in Verbindung gebracht werden können oder um solche, denen Prozesse vom Typ 2 zu Grunde zu liegen scheinen. Diese Dichotomie erscheint in gewisser Weise artifiziell und ist dem Umstand geschuldet, dass die vorgestellten Theorien ihren Fokus nicht auf die Unterscheidbarkeit von Verarbeitungsprozessen, sondern eben auf die Erklärung bestimmter kausaler Phänomene legen. Die vorgestellten Theorien sollten nicht den Eindruck erwecken, als würden sie grundsätzlich von kausaler Verarbeitung im Rahmen von Typ 1- bzw. Typ 2Prozessen ausgehen. Vielmehr sollten sie der Verdeutlichung der Annahme dienen, dass unterschiedliche kausale Urteile mit unterschiedlichen Arten von Verarbeitungsprozessen erklärt werden können.

Im nun folgenden Abschnitt werden einige kausale Theorien vorgestellt, die mit der Vorstellung des Wirkens beider Typen von Prozessen verbunden werden können. Bei diesen Theorien handelt es sich einerseits um die Theorie der kausalen Verarbeitung von White (1989), die die Operation sowohl von automatischen Typ 1-Prozessen als auch kontrollierten Typ 2-Prozessen bei der kausalen Informationsverarbeitung annimmt. Zweitens wird auf ein Zwei-Prozess-Modell des kausalen Schließens von Fugelsang und Thompson (2003) eingegangen, das von einer automatisch und unbewusst ablaufenden ersten Phase der Rekrutierung von Vorwissen ausgeht, die von einer zweiten, bewusst und kontrolliert ablaufenden Phase der Evaluation der empirischen Evidenz gefolgt wird. Zuletzt wird der Ansatz von Verschueren, Schaeken und d'Ydewalle (2005) vorgestellt, der davon ausgeht, dass zwischen heuristischen (Typ 1-) und analytischen (Typ 2-) Prozessen beim konditionalen kausalen Schließen unterschieden werden kann. 


\subsubsection{Die Theorie der kausalen Verarbeitung (White, 1989)}

Eine frühe Theorie der kausalen Informationsverarbeitung, die neben Aspekten der kausalen Intuition auch solche der kausalen Deliberation aufnimmt, stammt von Peter White (1989). Er betont, dass es in seiner Theorie nicht darum geht, die Natur tatsächlich existierender Kausalbeziehungen in der Welt, sondern das von Menschen geteilte alltägliche Verständnis kausaler Verbindungen zu untersuchen. In seiner Causal Powers Theory of Causal Processing geht White davon aus, dass Menschen kausale Informationen in Abhängigkeit von verschiedenen Faktoren sowohl mittels Typ 1- als auch mittels Typ 2Systemen verarbeiten. Die Theorie basiert auf der Annahme der Existenz eines allgemein geteilten Konzeptes von Kausalität, in dem generative Mechanismen die Erzeugung von Effekten durch ihre Ursachen ermöglichen. Der Vorgang, durch den eine Ursache einen Effekt erzeugt wird als die Freisetzung einer kausalen Kraft (causal power) interpretiert, die unter bestimmten auslösenden Bedingungen (releasing conditions) erfolgt. Diese auslösenden Bedingungen treten dabei oft in Form von Ereignissen auf. So ist beispielsweise beim Lackmustest der Vorgang des Vermischens von Lackmus-Tinktur und zu testender Flüssigkeit die auslösende Bedingung. Die Fähigkeit von Säure (mit einem pH-Wert von kleiner als 4,5), Lackmus rot zu färben, stellt hingegen eine kausale Kraft dar. Die kausale Kraft wird als eine stabile Eigenschaft von Dingen (wie physischen Objekten oder Personen) verstanden. Sie wirkt jedoch nicht direkt auf den Effekt, sondern vermittelt durch eine Übertragung (transmission) einer Kraft oder Energie von der Ursache auf den Effekt (White, 2009). Neben der kausalen Kraft ist in diesem Konzept von Kausalität noch die ebenfalls als Eigenschaft verstandene Empfänglichkeit (liability) eines Objektes von Bedeutung. Die Empfänglichkeit kann als Fähigkeit verstanden werden, bestimmten Veränderungen unterzogen werden zu können. So ist nach White (1989) die Formbarkeit von Kupfer im Sinne einer Empfänglichkeit für bestimmte Veränderungen in der äußeren Gestalt nach Bearbeitung mit einem Hammer zu verstehen. Im oben genannten Beispiel des Lackmustests ist die Fähigkeit der Lackmus-Tinktur sich zu verfärben als Empfänglichkeit zu verstehen. In einer konkreten Situation wird das soeben vorgestellte basale Kausalitäts-Konzept mit Kontext-spezifischen Annahmen gefüllt.

White (1989) nimmt an, dass kausale Verarbeitung auf Grundlage generativer Beziehungen zwischen Ursache und Effekt über einen Entwicklungsprozess in der Kindheit erlernt wird. Zunächst würden Kinder kausale Verarbeitung auf der Basis eines Eigenschaftstransfers zwischen zwei Objekten durchführen. Die Grundlage dieses Transfers 
bilden Wahrnehmungen von kontinuierlichen Bewegungscharakteristika, die sich über zwei als getrennt wahrgenommene Objekte ausdehnen. Dabei besteht eine kontinuierliche Beziehung wenn die kausalen Cues zeitliche Kontiguität, räumliche Kontiguität, zeitlicher Vorrang der ,Ursache“ vor dem ,Effekt‘ und Ähnlichkeit der kausal relevanten Eigenschaften vor und nach dem Transfer (vgl. Abschnitt 2.1) vorliegen.

Automatische kausale Verarbeitung findet nach White (1989) beispielsweise (aber nicht ausschließlich) im Bereich der wahrgenommenen Kausalität statt. Wird z.B. beobachtet, wie ein Hammer ein Glas zerschlägt, so wird der Hammerschlag automatisch als Ursache für die Zerstörung des Glases wahrgenommen. In diesem Fall ist die automatische kausale Verarbeitung somit ein Teil automatisch ablaufender perzeptueller Prozesse. White identifiziert zwei Voraussetzungen für automatisch ablaufende kausale Verarbeitung. Zum einen muss ein basales Konzept von Kausalität, wie das der oben erläuterten generativen Mechanismen, vorhanden sein. Zum anderen ist die Existenz Kontext-spezifischer Annahmen über die eine Situation betreffenden kausalen Kräfte, Empfänglichkeiten, auslösenden Bedingungen und möglichen Effekte vonnöten. White zufolge erwerben Menschen Wissen über die kausalen Kräfte einer Vielzahl von Dingen bzw. Arten von Dingen. Dieses Wissen kann ebenso wie das Wissen über bestimmte auslösende Bedingungen oder mögliche Effekte mit zunehmender Vertrautheit im Rahmen des geschilderten automatischen kausalen Verarbeitungsprozesses genutzt werden. Der Erwerb von Wissen über kausale Kräfte erfolgt dabei entweder durch die Abstrahierung von über kausale Cues (s.o.) erworbenen Informationen oder durch direktes Lernen.

Automatische kausale Verarbeitung wird von White (1989) als der Verarbeitungsstandard angesehen. Eine kontrollierte kausale Verarbeitung (im Sinne eines Typ 2-Prozesses) von Ereignissen, die prinzipiell auch automatisch verarbeitet werden können, findet der Theorie zufolge nur statt, wenn entweder die kausalen Kräfte, die Empfänglichkeiten, die auslösenden Bedingungen oder eine Kombination dieser Faktoren in den Fokus der Aufmerksamkeit gerückt werden. Dies geschieht im Rahmen der bewusst ablaufenden Identifikation von Ursachen eines eingetretenen Ereignisses. Darüber hinaus können kontrollierte kausale Verarbeitungsprozesse auch stattfinden, wenn eine automatische kausale Verarbeitung nicht möglich ist, da ein Ereignis z.B. unerwartet auftritt oder unbekannt ist. White schlägt in diesem Zusammenhang zwei Regeln vor, die das Auftreten kontrollierter kausaler Verarbeitung in einem Individuum bestimmen, das sich in einer Situation befindet, in der eine automatische kausale Verarbeitung nicht möglich ist. Zum einen muss das Ergebnis 
der kausalen Verarbeitung (die Identifikation einer Ursache) eine praktische Relevanz (practical concern) für das Individuum besitzen. Andererseits muss dem Individuum die zu identifizierende Ursache als mögliche Ursache des zu erklärenden Effektes bekannt und nicht durch verfügbare Informationen (zu Recht oder $\mathrm{zu}$ Unrecht) ausgeschlossen sein (applicability of an existing causal belief). Ein für die verarbeitende Person adäquates Ergebnis kontrollierter kausaler Verarbeitung basiert somit erstens auf einer allgemeinen Konzeption einer kausalen Verbindung als generativer Beziehung, die das Wirken einer kausalen Kraft einer Entität repräsentiert. Zweitens beinhaltet es die Identifikation einer spezifischen kausalen Kraft, auslösenden Bedingung oder Empfänglichkeit (bzw. einer Kombination einiger oder aller dieser Konzepte) als in Frage kommende Ursache. Drittens wird es durch praktische Belange gesteuert und trägt zu ihrer Erfüllung bei. Viertens ist die identifizierte Ursache bereits als mögliche Ursache des zu erklärenden Effektes bekannt und wird nicht durch vorhandene Informationen als Ursache ausgeschlossen.

Zusammenfassend nimmt der Ansatz von White (1989) eine Trennung von Typ 1- und Typ 2-Prozessen der kausalen Informationsverarbeitung vor. Allerdings geht aus der Theorie nicht eindeutig hervor, ob die Ergebnisse der automatischen kausalen Verarbeitung bewusstseinsfähig sein können, da White davon ausgeht, dass bereits die Identifikation von Ursachen Prozesse vom Typ 2 erfordert: „What people report as the cause is whatever is identified in controlled, not automatic, causal processing, simply because it is that to which attention is given“" (White, 1989, S. 442). Diese Aussage legt nahe, dass White Aufmerksamkeit als das wesentliche Unterscheidungsmerkmal zwischen automatischer und kontrollierter kausaler Verarbeitung ansieht. In diesem Fall wäre es dann nicht möglich, dass Ergebnisse automatischer Verarbeitungsprozesse ins Bewusstsein gelangen..Somit wären auch die für die vorliegende Arbeit relevanten Aspekte kausalen Denkens der Theorie von White zufolge unweigerlich mit Prozessen vom Typ 2 verbunden. Whites Standpunkt stünde damit im Widerspruch zur z.B. von Kahneman und Frederick (2002) vertretenen Ansicht, dass Ergebnisse von Typ 1-Prozessen sehr wohl bewusst zugänglich sein können, während der Prozess an sich nicht bewusst wahrnehmbar ist. Trotz dieser Einschränkung ist die Theorie der kausalen Verarbeitung von White (1989) von Bedeutung für die vorliegende Arbeit, da sie nach dem Wissen des Autors die erste kausale Theorie darstellt, die davon ausgeht, dass kausale Informationen sowohl mittels automatischer (Typ 1-) Prozesse als auch mittels kontrolliert ablaufender (Typ 2-) Prozesse verarbeitet werden können. 


\subsubsection{Der Zwei-Prozess-Ansatz von Fugelsang und Thompson (2003)}

Fugelsang und Thompson (2003) schlagen auf der Basis eigener empirischer Untersuchungen ein Zwei-Prozess Modell kausalen Schließens vor. Dabei gehen sie davon aus, dass kausales Vorwissen in einer ersten Phase automatisch und unbewusst (also mittels Typ 1-Prozessen) für die als möglich erachteten Ursachen eines aufgetretenen Effektes rekrutiert wird. Auf dieser Grundlage wird in einer zweiten Phase die vorliegende empirische Evidenz, die in der Studie von Fugelsang und Thompson stets aus Daten über die Kovariation zweier Variablen besteht, mittels bewusster (Typ 2-) Verarbeitungsprozesse evaluiert.

Die Nutzung kausalen Wissens über Typ 1-Prozesse wurde bereits in der Theorie von White (1989) angenommen. Automatisch nutzbares kausales Wissen besteht White zufolge aus einem generellen Konzept generativer Beziehungen sowie spezifischeren Annahmen über kausale Kräfte, auslösenden Bedingungen und Empfänglichkeiten, durch die Ursachen ihre Effekte hervorrufen. Fugelsang und Thompson (2003) zählen die Theorie Whites` zu den mechanismus-basierten Ansätzen. In ihrer eigenen Theorie gehen sie jedoch davon aus, dass neben Mechanismus-basierten Kausalwissen auch im Langzeitgedächtnis gespeicherte Repräsentationen von Kovariationen mittels Typ 1-Prozessen verarbeitet werden können. Die Rekrutierung der beiden Arten von kausalem Vorwissen wird als ein heuristischer Prozess verstanden. Des Weiteren nehmen die Autoren an, dass Mechanismus-basiertes und Kovariations-basiertes Kausalwissen unabhängig voneinander existieren, bei der automatischen Verarbeitung aber auch interagieren können (für eine umfassende Kritik an der Annahme der Unabhängigkeit der beiden Arten von Kausalwissen siehe Perales, Shanks \& Lagnado, 2010). Als Beleg für den unbewussten Charakter der beschriebenen Prozesse führen Fugelsang und Thompson (2003) an, dass die Versuchspersonen in ihren Experimenten weitestgehend im Unklaren darüber waren, wie stark ihre Urteile durch Vorwissen beeinflusst wurden. Die unbewusste Rekrutierung von Vorwissen über eine potenzielle Ursache eines zu erklärenden Effektes resultiert nach Fugelsang und Thompson in einer Bewertung der Glaubwürdigkeit (believability). Dieser Begriff bezieht sich auf das Vermögen der potenziellen Ursache, den betrachteten Effekt tatsächlich hervorzurufen. Eine hohe Glaubwürdigkeit ist unabhängig von der Stärke der vorliegenden empirischen Evidenz mit höheren Urteilen bezüglich der Wirksamkeit der möglichen Ursache verbunden.

Die zweite Phase des Modells von Fugelsang und Thompson (2003) besteht in der bewussten Evaluation der empirischen Evidenz. Die Autoren gehen davon aus, dass die Entscheider in dieser Phase die empirische Evidenz bewusst und gründlich evaluieren und 
anschließend die wahrgenommene Wirksamkeit (perceived causal efficacy) der potenziellen Ursache bewerten. Die Annahme bewusster Informationsverarbeitungsprozesse in dieser Phase wurde in den durchgeführten Studien durch das Vermögen der Versuchspersonen, den Einfluss der empirischen Evidenz auf ihre Urteile tendenziell richtig einzuschätzen, gestützt. Ein zentrales Ergebnis der von Fugelsang und Thompson durchgeführten Studien ist, dass die Herkunft des Vorwissens einen Einfluss auf die bewusste Verarbeitung in Phase 2 ausübt. Kovariations-basiertes Vorwissen und Kovariations-basierte empirische Evidenz tragen additiv zum Kausalurteil bei. Dagegen besteht zwischen Mechanismus-basiertem Vorwissen und Kovariations-basierter Evidenz eine Interaktion: Kovarianz-basierte Daten für potenzielle Ursachen mit hoher Glaubwürdigkeit werden stärker gewichtet als Kovarianz-basierte Daten für potenzielle Ursachen mit niedriger Glaubwürdigkeit, wenn die jeweilige Glaubwürdigkeit aus Mechanismus-basiertem Vorwissen abgeleitet wurde. Somit bestimmen zwei Komponenten der automatischen Prozesse der Phase 1 den Ablauf der bewussten Prozesse in Phase 2: Zum einen hat die Glaubwürdigkeit der potenziellen Ursache unabhängig von der Art des Vorwissens, auf dem sie basiert, einen direkten Effekt auf das finale Kausalurteil. Zum anderen interagiert Glaubwürdigkeit, die auf Grundlage von Mechanismus-basiertem Vorwissen entsteht, mit der empirischen Evidenz in Bezug auf das finale Kausalurteil. Kausales Vorwissen beeinflusst also die deliberativen und analytischen (Typ 2-) Prozesse, die zur Evaluation der empirischen Evidenz nötig sind.

Insgesamt stellt der Ansatz von Fugelsang und Thompson (2003) kausale Urteile somit als zweistufigen Vorgang dar, der sowohl Prozesse von Typ 1 als auch solche vom Typ 2 beinhaltet. Dabei sind die Ergebnisse der automatisch und unbewusst ablaufenden ersten Phase jedoch nicht als eigenständige Urteile, sondern vielmehr als eine Art (unbewusster) Input für die bewusst ablaufenden Prozesse in der zweiten Phase, in der das finale Urteil gebildet wird, zu verstehen. Dennoch kann der Ansatz in die Kategorie der Theorien der kausalen Intuition und Deliberation eingeordnet werden, da die Resultate der Typ 1-Prozesse in Phase 1 als kausale Intuitionen verstanden werden können, die einen unbewussten Einfluss auf die Typ 2-Prozesse der kausalen Deliberation in Phase 2 ausüben.

\subsubsection{Der Zwei-Prozess-Ansatz von Verschueren, Schaeken und d’Ydewalle (2005)}

Einen weiteren Zwei-Prozess-Ansatz liefern Verschueren, Schaeken und d'Ydewalle (2005) mit ihrer Unterscheidung von heuristischen und analytischen Prozessen beim kausalen konditionalen Schließen (causal conditional reasoning). Sie untersuchen Annahmen über Informationsverarbeitungsprozesse und differenzieren dabei zwischen probabilistischen 
Ansätzen und einem auf mentalen Modellen basierendem Ansatz. Verschueren und Kollegen identifizieren den Kern des probabilistischen Ansatzes darin, dass die Wahrscheinlichkeit, eine Konklusion zu akzeptieren mit der Wahrscheinlichkeit der Kovariation von Ursache und Effekt in Beziehung steht. Die Wahrscheinlichkeit der Kovariation dient hierbei als ein Maß der Glaubwürdigkeit bzw. Unsicherheit der konditionalen Aussage. Hingegen steht beim Ansatz der mentalen Modelle die Verfügbarkeit von Gegenbeispielen für eine konditionale Aussage, die die Konklusion akzeptieren, im Fokus. Diese Gegenbeispiele können entweder Situationen sein, in denen der Effekt trotz Anwesenheit der Ursache nicht auftritt (disablers) oder Situationen, in denen eine andere als die betrachtete Ursache den Effekt bewirkt (vgl. Cummins et al., 1991; Abschnitt 2.4.3.1). Verschueren et al. (2005) gehen ferner davon aus, dass heuristische (Typ 1-) Prozesse die Verarbeitung probabilistischer Informationen charakterisieren, während analytische (Typ 2-) Prozesse für die Verarbeitung mentaler Modelle verantwortlich sind. Heuristische Prozesse werden von Verschueren und Kollegen in Übereinstimmung mit der in Abschnitt 2.2.2 dargestellten Sicht als Prozesse im System 1 charakterisiert. Sie sind somit als schnell, automatisch, weitgehend unbewusst und mit geringen Anforderungen an die Rechenkapazität zu verstehen. Analytische Prozesse laufen hingegen im System 2 ab und sind damit langsam, strategisch und bewusst. Es wird angenommen, dass analytische Prozesse die Ergebnisse heuristischer Prozesse überschreiben können und somit eine eindeutige Hierarchie zwischen beiden Prozesstypen besteht.

Verschueren et al. (2005) schlagen eine minimalistische Spezifikation probabilistischer Schlussfolgerung vor, in der sie davon ausgehen, dass die Likelihood, dass die Ursache vom Effekt gefolgt wird (L(e|c)) mit der Akzeptanz der Konklusionen bei Aufgaben, die auf den Schlussregeln Modus ponens und Modus tollens basieren, korreliert ist. Bei Aufgaben, die auf den Fehlschlüssen Affirmation der Konsequenz und Verneinung des Antezedens basieren, ist hingegen die Likelihood, dass dem Effekt die Ursache vorangeht (L(c|e)) mit der Akzeptanz der Konklusionen korreliert. Dabei gehen die Autoren davon aus, dass die Likelihoods in einem heuristischen Prozess auf der Grundlage von im Langzeitgedächtnis gespeicherten Situationen abgeleitet werden.

Der Theorie der mentalen Modelle (vgl. Johnson-Laird, Byrne \& Schaeken, 1992) zufolge besteht der Schlussfolgerungsprozess aus drei nacheinander ablaufenden Phasen. In der ersten Phase der Modellkonstruktion wird der Inhalt der Prämissen einer konditionalen Aussage explizit repräsentiert. Anschließend wird in einer zweiten Phase ein erster Schluss auf Basis einer begrenzten Auswahl expliziter mentaler Modelle gezogen. In der 
abschließenden dritten Phase wird dann dieser Schluss validiert, indem bewusst nach Gegenbeispielen gesucht wird. Der sequenzielle Ablauf und die bewusste Suche nach Gegenbeispielen dienen Verschueren et al. (2005) als Hinweise für das Vorliegen von analytischen Typ 2-Informationsverarbeitungsprozessen. Nach dem Ansatz der mentalen Modelle sollte die Akzeptanz der Konklusionen von Aufgaben, die auf Modus ponens und Modus tollens basieren, mit der Anzahl der vorstellbaren Bedingungen korreliert sein, die den Effekt verhindern können (disabling conditions). Hingegen sollte sich für Aufgaben, die auf der Affirmation der Konsequenz bzw. der Verneinung des Antezedens basieren eine Korrelation zwischen der Anzahl alternativer Ursachen und der Akzeptanz der Konklusion finden lassen.

Verschueren et al. (2005) testeten ihre Vorhersagen in mehreren Experimenten. Dabei sammelten sie Belege für den von ihnen propagierten Zwei-Prozess-Ansatz. Zum einen wurden Belege für die Annahme berichtet, dass heuristische Inferenzen auf Basis probabilistischer Informationen weniger Zeit in Anspruch nehmen als analytische Inferenzen auf Basis von Informationen über Gegenbeispiele. Zweitens konnte gezeigt werden, dass das Überschreiben von Ergebnissen des heuristischen Prozesses mit der assoziativen Stärke der analytisch inferierten Gegenbeispiele für eine Aussage in Beziehung steht: Je einfacher Gegenbeispiele gefunden werden konnten (d.h. je größer deren assoziative Stärke ist), desto größer war die Dominanz des analytischen Schlussfolgerungsprozesses. Darüber hinaus zeigte sich, dass die Versuchspersonen sowohl Informationen über die Likelihood als auch über Gegenbeispiele nannten, wenn sie aufgefordert wurden während des Schlussfolgerungsprozesses laut zu denken. Dieses Ergebnis wird von den Autoren als Beleg für die Existenz beider Arten von Prozessen gewertet. Wurden bei einer Aufgabe von einer Versuchsperson beide Arten von Informationen genannt, so folgten die Nennung der Gegenbeispiele meistens der Nennung der auf die Likelihood bezogenen Informationen. Diese Reihenfolge wird als weiteres Indiz für die unterschiedliche Geschwindigkeit der beiden Prozess-Arten angesehen.

Zusammenfassend postulieren Verschueren und Kollegen (2005) somit die Existenz zweier unterschiedlicher Schlussfolgerungsmechanismen für kausale Konditionale. Zum einen existiert ein heuristischer Mechanismus, der auf Informationen über die Likelihood der zu untersuchenden Aussage basiert, schnell abläuft und kaum Arbeitsgedächtniskapazität in Anspruch nimmt. Er läuft weitgehend unbewusst ab, nur sein Ergebnis gelangt ins Bewusstsein. Der heuristische Prozess verfügt somit über die typischen Eigenschaften von 
Typ 1-Prozessen. Zum anderen wird ein analytischer Schlussfolgerungsprozess angenommen, der auf Informationen über Gegenbeispielen der zu untersuchenden Aussage basiert, langsamer abläuft und höhere Anforderungen an die Kapazität des Arbeitsgedächtnisses stellt. Sowohl der Prozess des Abrufs von Gegenbeispielen als auch das finale Ergebnis des Prozesses sind bewusst. Somit ist der analytische Prozess eindeutig als Typ 2-Prozess zu verstehen. Zwischen den beiden Arten von Prozessen gibt es darüber hinaus eine klare Hierarchie, da der analytische Prozess in der Lage ist, das Ergebnis des heuristischen Prozesses zu überschreiben. Voraussetzung dafür ist lediglich, dass genügend Zeit für den analytischen Prozess zur Verfügung steht und ein eindeutiges Ergebnis auf analytischem Wege gefunden werden kann (d.h. es dürfen nicht zwei oder mehr mögliche Lösungen des selben Problems existieren).

\subsubsection{Agnostische Ansätze}

Nachdem in den vorangegangenen Abschnitten kausale Theorien vorgestellt wurden, die sich mehr oder weniger direkt in die Zwei-Prozess-Sichtweise einordnen ließen, sollen nun einige kausale Ansätze vorgestellt werden, die agnostisch in Bezug auf die Art der ihnen zu Grunde liegenden Prozesse sind. Die meisten kausalen Theorien sind dieser Kategorie zuzuordnen, da ihr Fokus auf der Erklärung bestimmter kausaler Urteile bzw. Inferenzen und nicht auf dem Bezug zu den Theorien kognitiver Prozesse liegt. Im Rahmen der vorliegenden Arbeit sollen nur einige wenige kausale Theorien exemplarisch für die Vielzahl der agnostischen Ansätze vorgestellt werden. Zunächst wird das Modell der Kausalattribution von Einhorn und Hogarth (1986) aufgrund seiner weitreichenden Bedeutung für das Feld der psychologischen Kausalitätsforschung eingehender betrachtet. Im Anschluss wird mit dem dynamische Modell von Wolff (2007) ein relativ aktueller Ansatz vorgestellt, der Attributionen mit Bezug zu kausalen Urteilen (wie Verursachung oder Ermöglichung) auf das Wirken verschiedener Kräfte zurückführt. Drittens werden Ansätze und Phänomene im Bereich der kausalen Kategorisierung (Ahn et al., 2000; Rehder , 1999, 2003; Rehder \& Kim, 2006,2010) betrachtet, da dieser Bereich für die im empirischen Teil der vorliegenden Arbeit vorgestellten Untersuchungen von großer Bedeutung sind. Abschließend wird auf die Causal Model Theory (Waldmann, 1996; Waldmann \& Holyoak, 1992; Waldmann et al., 1995) eingegangen, die als Grundlage anderer in dieser Arbeit vorgestellte Theorien angesehen werden kann. 


\subsubsection{Das Modell der Kausalattribution von Einhorn und Hogarth (1986)}

Einhorn und Hogarth (1986) entwickelten ein sehr elaboriertes Prozessmodell kausalen Schließens. Es versucht zu erklären, welche Faktoren die Evaluation potenzieller Ursachen beeinflussen. Dabei gehen sie ähnlich wie z.B. Kahnemann (2011) davon aus, dass Menschen die Tendenz besitzen, Ursachenzuschreibungen vorzunehmen, um Ereignisse in ihrer Umwelt zu verstehen. Das Resultat dieses Prozesses ist nicht deterministisch, sondern liegt in der Bestimmung der wahrscheinlichsten Ursache eines beobachteten Ereignisses. Die Autoren nehmen an, dass Menschen Kausalattributionen nicht streng nach den logischen Kriterien der Notwendigkeit und Suffizienz durchführen. Stattdessen schreiben sie Ursachen $\mathrm{zu}$, wenn die sog. INUS-Bedingung $(, X$ is an insufficient but necessary part of a complex scenario ( $\alpha$ ), which is itself wnnecessary but sufficient for $Y$ (INUS for short) ", Einhorn \& Hograth, 1986, S. 7) erfüllt ist.

Im Modell von Einhorn und Hogarth (1986) spielt das kausale Feld (causal field), in dem die Ursachenzuschreibung stattfindet, eine wichtige Rolle. Es bildet den Hintergrund vor dem sich das zu bewertende Ereignis abspielt und beeinflusst sowohl die Motivation, nach einer Ursache zu suchen, als auch das Spektrum der als möglich betrachteten Ursachen und damit wiederrum die Salienz der momentan fokussierten möglichen Ursache. Das Verhältnis, in dem ein Ereignis zum kausalen Feld steht, beeinflusst den Bedarf nach einer kausalen Erklärung: Hebt sich ein Ereignis deutlich vom kausalen Feld ab, so wird dadurch der Bedarf erhöht. Fügt es sich hingegen problemlos in das kausale Feld ein, so ist der Bedarf gering. Die konkrete Ausgestaltung des kausalen Feldes beeinflusst, welche Ursachen überhaupt als möglich in Betracht gezogen werden. Einhorn und Hogarth erläutern dies am Beispiel der Erklärung einer Krebserkrankung eines Chemiearbeiters. Wird diese vor dem Hintergrund seiner Arbeitstätigkeit gesehen, so werden andere Erklärungen als wahrscheinlich angesehen, als vor dem Hintergrund einer Familiengeschichte von Krebserkrankungen. Auch die Gewichtung von Informationen kann durch Veränderungen des kausalen Feldes beeinträchtigt werden. Ist beispielsweise bekannt, dass die Rate an Krebserkrankungen in der Firma des Betroffenen deutlich erhöht ist, so ist es von Bedeutung, ob diese Erhöhung relativ zu anderen Fabriken der chemischen Industrie insgesamt oder $\mathrm{zu}$ anderen Fabriken der chemischen Industrie, die die gleichen Produkte herstellen, besteht. In letzterem Fall wird verglichen mit dem ersten Fall mit einer höheren Wahrscheinlichkeit eine Kausalattribution auf Besonderheiten der Firma vorgenommen. 
Vor dem Hintergrund des kausalen Feldes werden im Modell von Einhorn und Hogarth (1986) vier Cues für die Existenz kausaler Beziehungen berücksichtigt: Kovariation, zeitliche Abfolge, Kontiguität und Ähnlichkeit. Diese Cues werden dem Modell zufolge als probabilistische Indikatoren kausaler Relationen verstanden. Menschen verwenden nach Möglichkeit mehrere Cues um sich gegen Urteilsfehler abzusichern und Unsicherheit zu reduzieren. Die normalerweise vorliegende Interkorrelation zwischen verschiedenen Cues in der Umwelt trägt zur Vermeidung dieser Fehler bei, indem sie einerseits die Folgen einer Vernachlässigung bestimmter Cues abschwächt und andererseits Aufmerksamkeit auf die Existenz weiterer Cues lenkt.

Informationen über die Kovariation einer möglichen Ursache A und einem Effekt B werden im Modell von Einhorn und Hogarth (1986) über die Häufigkeit der vier verschiedenen Kombinationsmöglichkeiten der An- bzw- Abwesenheit von A und B repräsentiert. Interessanterweise können nach dem Modell bereits einmalige Beobachtungen von Kovariation für kausale Interpretationen genutzt werden, da auch Kovariationen vor dem Hintergrund eines kausalen Feldes interpretiert werden. Wissen um das kausale Feld kann zur Einordnung des beobachteten Ereignisses genutzt werden. Des Weiteren können ähnliche, im Gedächtnis gespeicherte, Ereignisse abgerufen oder neue Ereignisse imaginiert werden. Auf diese Weise kann ausgehend von einer einzelnen Information über die Kovariation zweier Ereignisse die Wahrscheinlichkeit geschätzt werden, mit der das eine Ereignis das andere hervorruft. Diese Schätzung ist natürlich sehr anfällig für Fehler, sie kann aber als ein (erster) Indikator bei der Kausalattribution genutzt werden.

Die Beeinflussung kausaler Urteile durch temporale Informationen ist ein weiterer Cue für kausale Beziehungen im Modell von Einhorn und Hogarth (1986; siehe auch Abschnitt 2.1.2). Sie veranschaulichen dies anhand eines bekannten Beispiels von Tversky und Kahneman (1980): Die Wahrscheinlichkeit, dass eine Tochter blaue Augen hat, wenn bekannt ist, dass die Augenfarbe ihrer Mutter ebenfalls blau ist wird als höher eingeschätzt als die Wahrscheinlichkeit, dass die Mutter blaue Augen hat, wenn bekannt ist, dass ihre Tochter ebenfalls blaue Augen hat. Statistisch sind die Wahrscheinlichkeiten in beiden Fällen gleich hoch. Dennoch wird die Wahrscheinlichkeit des Auftretens eines Effektes (Augenfarbe der Tochter) bei einer gegebenen Ursache (Augenfarbe der Mutter) höher eingeschätzt als die Wahrscheinlichkeit des Vorliegens der Ursache bei gegebenem Effekt. Auch dieser Cue kann allerdings fehlerbehaftet sein, wenn z.B. die Information über das Eintreten des Effektes den Entscheider vor der Information über die An- oder Abwesenheit der Ursache erreicht. 
Im Modell von Einhorn und Hogarth (1986) wird weiterhin berücksichtigt, ob eine räumliche und bzw. oder zeitliche Kontiguität (vgl. Abschnitt 2.1.3) zwischen möglicher Ursache und Effekt besteht. Kontiguität lenkt nach Ansicht der Autoren Aufmerksamkeit auf Kontingenzen zwischen den beteiligten Variablen und somit auch auf die Bedeutung dieser Kontingenzen für die Bestimmung von Kausalität. Ist die räumliche und/oder zeitliche Kontiguität hingegen nicht bzw. nur schwer erkennbar oder liegt entgegen den situativen Erwartungen eine hohe Kontiguität vor, so wird die Attribution von Kausalität dadurch unwahrscheinlicher. Fehlende Kontiguität kann jedoch durch vorhandenes Wissen um (kausale) Verbindungen zwischen den Ereignissen ausgeglichen werden. Einhorn und Hogarth betonen, dass es zwischen Kontiguität und den anderen Cues besonders häufig zu Konflikten kommt, die durch Kompromisse aufgelöst werden müssen.

Zwei Arten von Ähnlichkeit beeinflussen im Modell von Einhorn und Hogarth (1986) die Kausalattribution. Zum einen besteht die Tendenz eher eine Kausalattribution vorzunehmen wenn sich mögliche Ursache und Effekt physisch ähnlich sind. Zum anderen führt eine Kongruenz in Dauer und Stärke zwischen möglicher Ursache und Effekt tendenziell zu stärkeren Kausalattributionen.

Neben den kausalen Cues und dem kausalen Feld kommt der Existenz einer kausalen Verbindung zwischen der möglichen Ursache und dem Effekt im Modell von Einhorn und Hogarth (1986) eine zentrale Bedeutung zu. Nur wenn eine direkte oder indirekte kausale Verbindung zwischen den beiden Ereignissen besteht (bzw. konstruiert werden kann) ist es möglich, eine Kausalattribution vorzunehmen. Mit anderen Worten ist das Bestehen einer kausalen Verbindung im Modell von Einhorn und Hogarth eine notwendige Bedingung für die Ursachenzuschreibung. Die Verbindung wird dabei als kausale Kette (causal chain) von einzelnen kausal miteinander verbundenen Ereignissen verstanden, die mit Hilfe von Vorwissen oder Imagination erstellt wird. Bei der Konstruktion der kausalen Kette müssen die Eigenschaften der kausalen Cues des Szenarios berücksichtigt werden.

Sowohl die Länge der Kette als auch die Stärke der Verbindungen zwischen ihren einzelnen Elementen übt einen großen Einfluss auf die Beurteilung der Stärke der Kausalbeziehung zwischen möglicher Ursache und Effekt aus. Die Stärke der Verbindung zwischen zwei Variablen wird nach Einhorn und Hogarth (1986) durch deren Kovariation bestimmt. Somit gilt: Je länger die Kette und je schwächer die Verbindungen, desto geringer wird die Wahrscheinlichkeit mit der der Effekt durch die mögliche Ursache hervorgerufen wird eingeschätzt. Formal werden bei der Berechnung der Stärke der gesamten Kette die 
Parameter für die Stärke der einzelnen Verbindungen miteinander multipliziert. Auch das kausale Feld beeinflusst die Stärke der kausalen Kette, da es bestimmt, wie genau die Beziehung zwischen möglicher Ursache und Effekt erklärt werden soll. Allgemeine bzw. oberflächliche Erklärungen benötigen dabei weniger lange Ketten als detaillierte Erklärungen. Einhorn und Hogarth betonen, dass die Stärke der kausalen Kette in ihrem Modell auch als Stärke einer zuvor erworbenen Theorie interpretiert werden kann, die Menschen beim kausalen Schließen benutzen. Diese Theorien basieren auf zuvor gemachten Erfahrungen oder Wissen über die Welt.

Die Einflüsse der verschiedenen vorgestellten Komponenten lassen sich nach Einhorn und Hogarth (1986) in folgender Formel zusammenfassen:

$$
s(X, Y)=Q_{T} Q_{B} Q_{L}\left(\lambda_{C} Q_{C}+\lambda_{S} Q^{\prime}{ }_{S}\right)
$$

Hierbei repräsentiert $\mathrm{s}(\mathrm{X}, \mathrm{Y})$ die Bruttostärke der kausalen Beziehung zwischen einer möglichen Ursache $X$ und dem Effekt $Y, Q_{T}$ die zeitliche Abfolge von X und $Y(0 ; 1), Q_{B}$ den Grad, zu dem X vor dem Hintergrund des kausalen Feldes hervortritt $\left(0 \leq Q_{B} \leq 1\right), Q_{L}$ die Stärke der kausalen Kette von X zu Y $\left(0 \leq Q_{L} \leq 1\right), Q_{C}$ die Kovariation zwischen X und Y und Q's die physikalische Ähnlichkeit von X und Y. $\lambda_{C}$ und $\lambda_{S}$ stellen Aufmerksamkeitsgewichte dar. Die Faktoren Kontiguität und Kongruenz von möglicher Ursache und Effekt in Dauer und Stärke fließen über ihren Einfluss auf $\mathrm{Q}_{\mathrm{L}}$ in das Modell ein.

Wie aus Gleichung (2) ersichtlich müssen $\mathrm{Q}_{\mathrm{T}}, \mathrm{Q}_{\mathrm{B}}$ und $\mathrm{Q}_{\mathrm{L}}$ Werte größer Null annehmen, damit s(X,Y) einen positiven Wert annehmen kann. Diese Faktoren müssen somit für eine Kausalattribution notwendigerweise vorhanden sein. Hingegen werden die Faktoren Kovatiation und physikalische Ähnlichkeit als kompensatorisch aufgefasst. Zusammen mit der zuvor erwähnten Möglichkeit, die kausale Kette $\mathrm{Q}_{\mathrm{L}}$ auch als bereits existierende kausale Theorie aufzufassen, ergeben sich verschiedene Möglichkeiten der Kompensation. So können beispielsweise fehlende Kovariationsinformationen durch eine existierende kausale Theorie $\mathrm{Q}_{\mathrm{L}}$ und/oder die Existenz einer physikalischen Ähnlichkeit zwischen möglicher Ursache und Effekt Q's kompensiert werden. Die aus Gleichung (2) ermittelte Bruttostärke wird abschließend gemäß der Anzahl und Salienz alternativer möglicher Ursachen diskontiert. Mit steigender Anzahl von Alternativerklärungen verringert sich die Notwendigkeit der im Fokus stehenden möglichen Ursache X für das Auftreten des Effektes Y. 
Einhorn und Hogarth (1986) machen keine Angaben darüber, ob ihr Modell eine bewusste Verarbeitung erfordert. Betrachtet man die einzelnen Komponenten ihres Modells, so ergibt sich ein heterogenes Bild. Die Position einer möglichen Ursache vor dem Hintergrund des kausalen Feldes legt beispielsweise das Wirken von Typ 2-Prozessen nahe, wenn davon gesprochen wird, dass ein deutlich vor dem Hintergrund hervortretendes Ereignis den Bedarf nach einer kausalen Erklärung steigert. Dagegen kann der Einfluss des kausalen Feldes auf die Menge der Variablen, die überhaupt als Ursache in Betracht kommen, potenziell sowohl über Typ 1- als auch über Typ 2-Prozesse vermittelt werden. Ähnlich verhält es sich mit der Verarbeitung der verschiedenen Cues kausaler Relationen. So ist es z.B. nicht klar, ob Informationen über die Kovariation von möglicher Ursache und Effekt mittels bewusster Typ 2-Prozesse verarbeitet werden, oder lediglich als Ergebnis unbewusster Typ 1-Prozesse ins Bewusstsein gelangen. Die Verarbeitung der zeitlichen Abfolge von möglicher Ursache und Effekt scheint aufgrund ihres nach Einhorn und Hogarth schnellen und oftmals unbewussten Ablaufs eher einen Typ 1-Prozess darzustellen. Hingegen ist die Konstruktion einer kausalen Kette schwerlich ohne Beteiligung von Typ 2-Prozessen vorstellbar. Somit scheinen beide Arten von Prozessen im Modell von Einhorn und Hogarth eine Rolle zu spielen. Da es aber nicht möglich ist abzugrenzen, welche Komponenten standardmäßig mittels Typ 1- und welche mittels Typ 2-Prozessen verarbeitet werden, wird das Modell der Klasse der agnostischen Ansätze zugeordnet.

\subsubsection{Das Force Dynamics Model von Wolff (2007)}

Die Untersuchung der Zuschreibung kausaler Begriffe auf Grundlage der Konfiguration verschiedener Kräfte steht im Mittelpunkt des nun vorgestellten Modells. Ausgehend vom Force-Dynamics-Modell Talmys (1988) versteht das dynamische Modell (dynamics model) von Wolff (2007) kausale Konzepte als Muster von Kräften und je einem Ortsvektor, der auf einen Endzustand weist. Damit unterscheidet es sich von anderen physikalistischen Modellen, die von der Übertragung einer kausalen Kraft von einer Ursacheauf eine Effekt-Variable ausgehen. Als Argument für die abweichende Auffassung von Kausalität nennt Wolff Situationen, in denen nichts passiert (also auch keine Übertragung einer Kraft vorliegt) und dennoch eine kausale Attribution stattfindet wie z.B. „Pressure will cause the water to remain liquid at slightly below $0^{\circ} \mathrm{C}^{\prime}$. Dennoch versteht Wolff sein Modell als physikalistisch. Es geht davon aus, dass sich mentale Repräsentationen kausaler Ereignisse am Ablauf dieser Ereignisse in der realen Welt orienteren. Das dynamische Modell identifiziert verschiedene Arten von Kräften, die die mentale Repräsentation kausaler 
Zusammenhänge determinieren. Die Kräfte bestehen generell aus den beiden Komponenten Richtung und Stärke. Das Modell geht davon aus, dass kausale Konzepte mittels dreier Dimensionen verstanden werden können. Die erste Dimension bildet die als Kraft verstandene Tendenz des Effektes (,Patient' im dynamischen Modell), einen bestimmten, für die Kausalattribution relevanten, Endzustand $\mathrm{zu}$ erreichen. Diese Tendenz kann in unterschiedlichen Abstufungen auf den Endzustand weisen oder ihm entgegengesetzt sein. Ein Effekt kann also von selbst dem Endzustand entgegen streben oder sich von ihm entfernen. Die zweite Dimension gibt Auskunft darüber, ob die Tendenz der UrsacheVariablen (,Affektor') und die Tendenz der Effekt-Variablen konkordant sind oder nicht. Die dritte Dimension im dynamischen Modell erfasst, inwiefern sich der Patient dem Endzustand letztlich (also nach Einwirken aller Kräfte) nähert.

Die von Wolff postulierte Theorie ist in der Lage, auch die Repräsentation von mit dem Konzept der Verursachung (cause) verwandten (aber nicht identischen) Konzepten zu erklären und vorherzusagen. So können die Konzepte Ermöglichung (enable) und Verhinderung (prevent) eines Effektes durch die Affektor-Variable dargestellt werden. Ein weiteres Konzept besteht im Erreichen des Endzustandes trotz gegenläufiger von der Affektor-Variablen ausgeübter Kraft (despite). Dabei geht die Theorie davon aus, dass die Kombination der verschiedenen berücksichtigten Faktoren bzw. Kräfte ausschlaggebend dafür ist, welches Konzept repräsentiert wird oder ob das Ereignis als nicht kausal eingestuft wird. Das Ereignis „A verursacht B“ ist beispielsweise dadurch charakterisiert, dass 1.) die Tendenz des Patienten dem Endzustand entgegengesetzt ist, 2.) die Tendenz des Affektors und des Patienten nicht konkordant sind (d.h. die Tendenz des Affektors weist in Richtung des Endzustandes) und 3.) der Endzustand erreicht wird. Im Modell werden die einzelnen Größen als Kraft-Vektoren (force vectors) aufgefasst und auch graphisch dargestellt. In mehreren Experimenten konnte Wolff zeigen, dass die Kausalurteile von Versuchspersonen den Vorhersagen des dynamischen Modells entsprechen.

Eine Erweiterung des dynamischen Modells von Wolff (2007) stellt das transitive dynamische Modell (Transitive Dynamics Model, Barbey \& Wolff, 2007) dar, das die Beschränkung auf die Repräsentation einzelner Kausalrelationen aufhebt und beschreibt, wie Kombinationen von Relationen (z.B. „A verusacht B“ und „B verursacht C“) über einen transitiven Schluss in eine Relation zwischen den Entitäten A und C überführt werden. Mit Hilfe des transitiven dynamischen Modells ist es somit möglich, die Bildung mentaler Repräsentationen komplexerer Kausalbeziehungen zu erklären. Dabei werden die beiden 
Relationen als Prämissen einer neu zu bildenden Konklusion verstanden und miteinander dadurch kombiniert, dass der aus der ersten Prämisse resultierende Vektor als Vektor des Affektors in der zweiten Prämisse genutzt wird. Die Richtung dieses Affektors entspricht der Richtung des aus der ersten Prämisse resultierenden Vektors unter der Voraussetzung, dass die Ausprägung des in beiden Prämissen auftretenden Ereignisses (hier: „B“) dieselbe ist. Dies ist im oben angegebenen Beispiel der Fall. Stehen hingegen die Ausprägungen des Ereignisses in Konflikt wie dies beispielsweise bei der Verknüpfung der Relationen „A

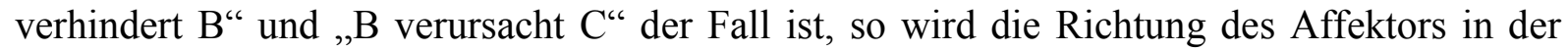
zweiten Prämisse invertiert. Der Konflikt entsteht im angeführten Beispiel dadurch, dass bei Vorliegen von A ,nicht-B“ eintritt und damit das in beiden Prämissen auftretende Ereignis „B“ unterschiedliche Ausprägungen aufweist. Der Affektor in der Konklusion der beiden Prämissen (im erstgenannten Beispiel „A verursacht $C^{(*)}$ ) entspricht dann dem Affektor der ersten Prämisse und der Vektor des Endzustandes der Konklusion dem Vektor des Endzustandes der zweiten Prämisse. Die Kombination der Vektoren der Patienten beider Prämissen ergibt den Vektor des Patienten in der Konklusion. Der resultierende Vektor der Konklusion errechnet sich dann aus der Kombination von Affektor- und Patienten-Vektor. Im Rahmen beider Modelle kann die Stärke und Ausrichtung der verschiedenen Vektoren beeinflussen, welche Schlüsse aus einer kausalen Situation gezogen werden können. Aus der Verknüpfung der Relationen „A verhindert B“ und „B verhindert C“ kann so beispielsweise entweder der Schluss „A erlaubt C“ oder „A verursacht C“ abgeleitet werden (Chaigneau \& Barbey, 2008).

Im Rahmen der vorliegenden Arbeit interessieren vor allem die Aussagen in der Beschreibung des dynamischen Modells (Wolff, 2007), die Rückschlüsse auf die Art der beteiligten Informationsverarbeitungsprozesse zulassen. So beschreibt Wolff, dass Menschen die verschiedenen Kraft-Vektoren in einer mentalen Struktur bzw. einem mentalen Schema ähnlich einem Freikörperbild (free-body image) repräsentieren. Dabei geht Wollf davon aus, dass Menschen die Richtung der verschiedenen Kräfte korrekt repräsentieren und integrieren. Hingegen wird die Stärke der einzelnen Kräfte nur ungenau repräsentiert, wobei relative Aussagen bezüglich der Stärke mehrerer Kräfte möglich sind. Welche Prozesse allerdings an der Entstehung dieser Repräsentation beteiligt sind, ob diese selbst bewusst oder unbewusst ist und wie die repräsentierten Vektoren schließlich miteinander kombiniert werden, um eine kausale Attribution ableiten zu können, wird in der Beschreibung der Theorie nicht näher spezifiziert. 
Einen konkreteren Hinweis auf die Beteiligung von Typ 1-Prozessen im Rahmen der Kausalattribution im dynamischen Modell liefert Wolff (2007) mit der spanning heuristic. Sie soll dafür sorgen, dass nur diejenigen Resultanten als durch die vorhandenen Kraft-Vektoren hervorgebracht angesehen werden, deren Vektor sich innerhalb des von Affektor-Vektor und Patienten-Vektor aufgespannten Raums befinden. Dies geschieht mittels einer groben Einschätzung, ob die Resultante durch die Addition der Vektoren der beteiligten Kräfte entstanden sein kann. Da nicht anzunehmen ist, dass der Vorgang der Vektor-Addition von vielen Menschen bewusst durchgeführt werden kann, liegt der Schluss auf die Beteiligung von Typ 1-Prozessen nahe. Zudem werden Heuristiken nach der in dieser Arbeit verwendeten Unterscheidung durch Prozesse in einem System vom Typ 1 verarbeitet (vgl. Abschnitt 2.2.2). Somit scheint zumindest ein Teil der im Rahmen des dynamischen Modells erforderlichen Prozesse in einem System vom Typ 1 verarbeitet zu werden. Es kann aber keine Aussage darüber gemacht werden, ob die anderen Komponenten des Modells ebenfalls in einem System vom Typ 1 verarbeitet werden können. Insgesamt kann festgestellt werden, dass eine Einordnung in die Typ 1-/Typ 2-Prozesse nicht im Fokus der Beschreibung des Modells steht. An einigen Stellen finden sich zwar Hinweise auf Prozesse, diese sind jedoch nicht ausreichend, um das Modell in eine andere als die Kategorie der agnostischen Theorien einzuordnen.

\subsubsection{Ansätze zur kausalen Kategorisierung}

Im Folgenden sollen einige Ansätze vorgestellt werden, die Kategorisierungen auf der Basis kausaler Modelle betrachten. Die Relevanz dieser Ansätze für die vorliegende Arbeit besteht zum eine darin, dass Kategorisierungen von einigen der bereits betrachteten Ansätzen (z.B. Kahneman \& Frederick, 2002; Sloman, 1996; Smith \& DeCoster, 2000) im Hinblick auf die ihnen zu Grunde liegenden kognitiven Prozesse unterschiedlich eingeordnet werden und daher diesbezüglich empirisch geprüft werden sollen. Zudem werden im empirischen Teil dieser Arbeit Kategorisierungsurteile auf der Basis vorhandenem Kausalwissens abgefragt, um die ihnen zu Grunde liegenden kognitiven Prozesse näher zu untersuchen.

Kausale Strukturen sind ein wichtiges Merkmal für das Verständnis von Kategorien und Wissen über diese Strukturen ermöglicht die Kategorisierung wahrgenommener Stimuli (vgl. Rehder, 2010 für einen Überblick über den Stand der Forschung zur kausalen Kategorisierung). Ein Beispiel für eine Theorie, die Kategorisierungen auf der Grundlage kausaler Modelle beschreibt ist die Causal Model Theory of Categorization (Rehder, 1999, 2003; für einen Ansatz, der das Erlernen von Kategorien mittels Kausalmodellen beschreibt 
siehe Waldmann et al., 1995). Sie geht davon aus, dass die Art in der Menschen die Struktur von Kategorien repräsentieren und für Entscheidungen nutzen mit Kausalmodellen in Form kausaler Bayes-Netze (vgl. Abschnitt 2.1) beschrieben werden kann. Die Kategorienzugehörigkeit eines Exemplars hängt von der Likelihood ab, mit der es vom kausalen Modell erzeugt werden kann. Mit anderen Worten geht die Theorie davon aus, dass Menschen bei Kategorisierungsentscheidungen beurteilen, wie wahrscheinlich das zu kategorisierende Exemplar durch die ihnen bekannten kausalen Gesetzmäßigkeiten der Kategorie erzeugt wurde. Die Struktur des Kausalmodells wird der Theorie zufolge auf Basis von Wissen über die Kategorie erzeugt, ohne dass hierfür tatsächliche Beobachtungen notwendig sind. Hieraus folgt das Vermögen der Theorie, eine Erklärung für die Kategorisierung bislang nicht beobachtete Exemplare anbieten $\mathrm{zu}$ können. Damit unterscheidet sich die Causal Model Theory of Categorization von alternativen Kategorisierungsansätzen, die Repräsentationen von Kategorien ausschließlich auf Basis von Beobachtungen bilden. Ein Beispiel eines solchen Ansatzes ist das ähnlichkeitsbasierte Generalized Context Model (Nosofsky, 1986). Es geht davon aus, dass Menschen beobachtete Exemplare einer Kategorie im Gedächtnis speichern und neue Stimuli auf Grundlage der Ähnlichkeit zu diesen bewerten. Die Ähnlichkeitsbeziehungen zwischen den Stimuli werden dabei als abhängig vom Kontext angesehen.

Ausgehend von der Annahme, dass Kategorisierungen auf der Grundlage kausaler Modelle möglich sind, wurde in verschiedenen Studien die Bedeutung unterschiedlicher kausaler Faktoren für Kategorisierungsurteile empirisch untersucht (vgl. Rehder, 2010). In typischen Experimenten zur kausalen Kategorisierung (z.B. Rehder, 2003) lernen die Versuchspersonen zunächst die Kausalstruktur einer Kategorie und beurteilen die Wahrscheinlichkeit, mit der die im Anschluss präsentierten Exemplare zu dieser gelernten Kategorie gehören. Die präsentierten Exemplare bestehen dabei aus einer bestimmten Konfiguration von Ausprägungen der Variablen des gelernten Kausalsystems.

Verschiedene theoretische Ansätze postulieren den Einfluss unterschiedlicher kausaler Faktoren auf Kategorisierungsurteile. Zwei dieser Ansätze sollen an dieser Stelle kurz betrachtet werden. Dabei geht es nicht um eine kritische Evaluation, wie sie z.B. Rehder und Kim (2010) und Rehder (2010) zugunsten des kausalen Kohärenzeffektes (s.u.) oder Mayrhofer und Rothe (in Druck) mit einem Erklärungsansatz auf Grundlage kausaler Modelle, der die Rolle der Typikalität der die kausalen Mechanismen konstituierenden Merkmale betont, vornehmen. Es sollen lediglich zwei Konzepte des Einflusses kausaler 
Faktoren auf Kategorisierungsurteile vorgestellt werden, da Kategorisierungen im empirischen Teil dieser Arbeit im Hinblick auf die ihnen zu Grunde liegenden Prozesse untersucht werden. Dabei werden die beiden Konzepte genutzt, um Vorhersagen bezüglich der von den Versuchspersonen abzugebenden Urteile zu generieren.

Der bereits in Abschnitt 2.4.2.3 vorgestellte kausale Statuseffekt (Ahn et al., 2000) besagt, dass die Position eines Merkmals in einer kausalen Struktur dessen Zentralität und damit die Bedeutung des Merkmals für Kategorisierungsurteile bestimmt. Dabei hat die Anbzw. Abwesenheit eines Merkmals mit hohem kausalen Status einen größeren Einfluss als die An- bzw. Abwesenheit eines Merkmals mit niedrigem kausalen Status. Mit anderen Worten gehen Ahn und Kollegen davon aus, dass Ursachen vermittelt über ihren höheren kausalen Status einen größeren Einfluss auf Kategorisierungsurteile ausüben als Effekte. Ahn und Kollegen machen keine Aussagen über die Art der kognitiven Prozesse (im Sinne der Typ1/Typ2-Unterscheidung), die dem kausalen Statuseffekt zu Grunde liegen. Einzig die Aussage, dass der kausale Status von Merkmalen einer Kategorie tief im Kategorisierungsprozess verwurzelt ist, kann als schwacher Hinweis auf die Beteiligung von Typ 1-Prozessen gewertet werden. Sie reicht jedoch bei weitem nicht für eine eindeutige Zuordnung zu einem Prozesstyp aus, weshalb der Ansatz von Ahn et al. zu den agnostischen Theorien gezählt wird.

Ein alternativer Ansatz zum Einfluss kausaler Faktoren auf Kategorisierungen beschäftigt sich mit dem kausalen Kohärenzeffekt (causal coherence effect, Rehder \& Kim 2006, 2010). Bei diesem Effekt werden nicht wie beim kausalen Statuseffekt den einzelnen Eigenschaften einer Kategorie spezifische Gewichte zugewiesen, sondern die Interaktionen zwischen den einzelnen Eigenschaften berücksichtigt (Rehder, 2010). Stehen zwei Eigenschaften einer Kategorie beispielsweise in einer kausalen Beziehung zueinander, so ist es (eine hinreichend starke Kausalbeziehung vorausgesetzt) zu erwarten, dass beide Eigenschaften kovariieren (also gemeinsam An- bzw. Abwesend sind). Hingegen weist der Fall eines abwesenden Effektes bei anwesender Ursache eine niedrige Kohärenz auf. Somit kommt es beim kausalen Kohärenzeffekt weniger auf die Position von Variablen in einer kausalen Struktur an als dies beispielsweise beim kausalen Statuseffekt der Fall ist. Der Fokus auf die Interaktionen in einer kausalen Struktur führt zu dem paradox anmutenden Fall, dass eine Instanziierung, bei der sämtliche Eigenschaften abwesend sind eine höhere Kohärenz (und damit eine höhere Kategorisierungswahrscheinlichkeit) aufweist als eine Instanz, bei der einige, kausal nicht direkt miteinander verbundene Eigenschaften anwesend sind (vgl. Rehder, 2003). Die kognitiven Prozesse, die dem kausalen Kohärenzeffekt zu Grunde liegen werden 
im Hinblick auf die Typ 1-/Typ 2- Unterscheidung nicht näher betrachtet. Rehder und Kim (2010) verweisen zwar darauf, intuitive Urteile der Kategorienzugehörigkeit zu untersuchen, machen aber hierzu keine weiteren Angaben. In dieses Bild lässt sich auch die Aussage Rehder's (2010) einfügen, dass weiterer Forschungsbedarf besteht, um die Art der Prozesse $\mathrm{zu}$ identifizieren, die bei Kategorisierungen unter Berücksichtigung kausalen Wissens ablaufen.

\subsubsection{Die Causal Model Theory}

Die Causal Model Theory, (Waldmann, 1996; Waldmann \& Holyoak, 1992; Waldmann et al., 1995) berücksichtigt die in Abschnitt 2.1 identifizierten Charakteristika kausaler Beziehungen. Sie geht davon aus, dass Menschen kausale Zusammenhänge mit kausalen Modellen repräsentieren und diese bei der Verarbeitung neuer Informationen nutzen. Der Fokus der ursprünglichen Theorie liegt zwar im Erlernen kausaler Strukturen und darauf basierenden Inferenzen, aber ihre Annahmen wurden inzwischen im Rahmen anderer Ansätze auch auf Urteils- und Entscheidungssituationen übertragen. So beschreibt die in Abschnitt 2.4.3.3 vorgestellte Causal Model Theory of Choice (Sloman \& Hagmayer, 2006; Hagmayer \& Sloman, 2009) die Wahl von Interventionen in kausale Strukturen in der Welt auf Grundlage von Kausalmodellen. Auch die Causal Model Theory of Categorization (Rehder, 1999, 2003; vgl. Abschnitt 2.4.5.3) greift auf die Annahmen der Causal Model Theory zurück. Aufgrund ihrer Bedeutung für im Rahmen dieser Arbeit betrachteten Theorien und der Rolle bei der Berücksichtigung der Charakteristika kausaler Beziehungen soll an dieser Stelle kurz auf die Annahmen zur Art der kognitiven Prozesse eingegangen werden, auf denen die Causal Model Theory basiert.

Waldmann und Holyoak (1992; Waldmann, 1996) beschreiben die Verarbeitungsprozesse im Rahmen der Causal Model Theory als eine Interaktion von topdown-Annahmen und der Verarbeitung des Lern-Inputs. Dabei kann der durch Beobachtungen generierte Input im Sinne von Informationen über die Kovariation der im kausalen Modell vorhandenen Variablen verstanden werden. Die Verarbeitung dieser gewissermaßen statistischen Informationen wird durch verschiedene von der Ausgestaltung des existierenden Kausalmodells abhängige Vorannahmen beeinflusst. Beispiele dieser topdown-Beeinflussungen sind die Annahmen über die Gerichtetheit kausaler Beziehungen oder die Berücksichtigung kausal relevanter Ko-Faktoren (vgl. Waldmann \& Hagmayer, 1995). Die Causal Model Theory (Waldmann, 1996; Waldmann \& Holyoak, 1992; Waldmann et al., 1995) macht jedoch keine Aussagen darüber, ob diese top-down-Einflüsse von kausalem 
Vorwissen über Typ 1- oder Typ 2-Prozesse vermittelt werden. Auch zur Art der Prozesse, die der Verarbeitung des Inputs $\mathrm{zu}$ Grunde liegen wird diesbezüglich keine Annahme gemacht. Auf den ersten Blick könnte die Annahme von Waldmann und Holyoak (1992; Waldmann et al., 1995), dass sich Menschen beim Aneignen von Wissen über neue Domänen von abstraktem Wissen über die Welt leiten lassen als Hinweis auf das Wirken von Typ 2Verarbeitungsprozessen angesehen werden, da die Verarbeitung abstrakten Wissens oft mit Typ 2-Prozessen assoziiert wird (vgl. Abschnitt 2.2.2). Als Beispiel für die Art von abstrakten Weltwissen, das im Rahmen der Causal Model Theory eine Rolle spielt, nennen Waldmann und Holyoak Wissen über die grundlegenden Charakteristika kausaler Beziehungen wie z.B. die temporale Priorität einer Ursache vor ihrem Effekt (vgl. Abschnitt 2.1.2). Es ist allerdings fraglich, ob die Berücksichtigung dieses Charakteristikums kausaler Beziehungen tatsächlich die Operation von Typ 2-Prozessen erfordert. Vielmehr ist es denkbar, dass eine derart grundlegende Eigenschaft auch automatisch mittels Typ 1-Prozessen verarbeitet werden kann.

In den Experimenten, mit denen Waldmann und Holyoak (1992; Waldmann, Holyoak und Fratianne, 1995) die Annahmen ihrer Theorie testeten wurden die Versuchspersonen explizit über die Struktur der für ihre Aufgabe relevanten Kausalmodelle in Kenntnis gesetzt. In anderen Studien (z.B. Hagmayer et al., 2010) wurde hingegen gezeigt, dass Menschen auch dann Wissen über die Struktur kausale Systeme erwerben, wenn sie lediglich die Reaktion des Systems auf von ihnen gewählte Interventionen beobachten. Somit können sich mentale Repräsentation in Form kausaler Modelle sowohl auf expliziter als auch auf impliziter Grundlage bilden. Welche Prozesse jedoch den Einfluss von vorhandenem Wissen über Kausalmodelle auf Urteile und Entscheidungen bewirken, ist dadurch nicht geklärt. Somit ist die ursprüngliche Causal Model Theory (Waldmann, 1996; Waldmann \& Holyoak, 1992; Waldmann et al., 1995) den agnostischen kausalen Theorien zuzuordnen. Damit weicht sie von der Einordnung der auf ihr basierenden Causal Model Theory of Choice (Sloman \& Hagmayer, 2006; Hagmayer \& Sloman, 2009; vgl. Abschnitt 2.4.3.3) ab, die aufgrund der Betonung mentaler Simulationsprozesse als Theorie der kausalen Deliberation klassifiziert wurde.

\subsubsection{Zusammenfassung}

Der vorangegangene Abschnitt beschäftigte sich mit der Frage, wie die von verschiedenen Ansätzen im Bereich des kausalen Urteilens und Entscheidens untersuchten Fragestellungen hinsichtlich der ihnen zu Grunde liegenden kognitiven Verarbeitungsprozesse in die Typ 1-/ Typ 2-Unterscheidung eingeordnet werden können. Aufgrund der relativ 
starken Trennung zwischen den beiden Forschungsbereichen wurden in den kausalen Theorien nur sehr selten explizite Aussagen zu den kognitiven Verarbeitungsprozessen im Sinne der Zwei-Prozess-Debatte gemacht. Daher musste die Zuordnung oftmals auf Grundlage von Interpretationen von Aussagen in den verschiedenen Theorien vorgenommen werden. Die Untersuchung resultierte in vier Gruppen, in die die analysierten kausalen Theorien eingeordnet werden konnten. Die erste Gruppe bilden diejenigen Theorien, die sich mit der Annahme von intuitiven Typ 1-Verarbeitungsprozessen als Basis von Urteilen und Entscheidungen über kausale Sachverhalte vereinbaren lassen. Hierzu gehören Ansätze zur wahrgenommenen Kausalität (vgl. Rips, 2011), Urteilsverzerrungen (Tverksy \& Kahneman, 1980, White, 2006a) und Kategorisierungsurteilen auf Grundlage kausalen Vorwissens (Luhmann et al., 2006).

Die zweite Gruppe bilden die Theorien, die von einer Notwendigkeit der Beteiligung von Typ 2-Verarbeitungsprozessen bei der Bewältigung der von ihnen untersuchten Sachverhalte ausgehen. Hierzu gehören die Arbeiten von Cheng \& Holyoak (1985), sowie Goldvarg und Johnson-Laird (2001), die kausale Schlussfolgerungsprozesse als Analogie zum logischen Schließen verstehen. Außerdem kann davon ausgegangen werden, dass Ansätze, in denen Interventionen in Kausalmodelle untersucht werden (Sloman \& Lagnado, 2005; Sloman \& Hagmayer, 2006; Hagmayer \& Sloman, 2009), deren Folgen mittels mentaler Simulationen (vgl. Lagnado, 2011) evaluiert werden können, die Beteiligung von Typ 2Prozessen für notwendig erachten.

Drittens gibt es einige kausale Ansätze, die davon ausgehen, dass sowohl Typ 1- als auch Typ 2-Prozesse am kausalen Urteilen und Entscheiden beteiligt sein können. Hierzu zählen die Theorie der kausalen Verarbeitung von White (1989), der zwischen automatischer und kontrollierter Verarbeitung unterscheidet. Außerdem gehört der Zwei-Prozess-Ansatz von Fugelsang und Thompson (2003), bei dem eine auf Typ 1-Prozessen basierende Phase der Rekrutierung von kausalem Vorwissen einer auf Typ 2-Prozessen beruhende Phase der Evaluation der empirischen Evidenz vorausgeht, zu dieser Gruppe. Des Weiteren wurde die Theorie von Verschueren et al. (2005) der dritten Gruppe zugeordnet, der zwischen heuristischen (Typ 1-) und analytischen (Typ 2-) Prozessen beim konditionalen kausalen Schließen unterscheidet.

Der vierten und letzten Gruppe wurden schließlich kausale Ansätze zugeordnet, die als agnostisch im Hinblick auf die Typ 1-/ Typ 2-Unterscheidung angesehen werden können. Hierunter befindet sich das Modell der Kausalattribution von Einhorn und Hogarth (1986), 
das verschiedene Einflussfaktoren auf die Evaluation potenzieller Ursache beschreibt, sowie das Force-Dynamics Model von Wolff (2007), in dem Beurteilung von Situationen mittels kausaler Begriffe auf Basis der Konfiguration verschiedener Kräfte analysiert wird. Schließlich können die Causal Model Theory (vgl. Waldmann 1996), die davon ausgeht, dass Menschen kausale Zusammenhänge in Form kausaler Modelle repräsentieren, sowie die aus ihr abgeleitete Causal Model Theory of Categorization (Rehder, 2003) zu dieser Gruppe gezählt werden.

Insgesamt kann festgehalten werden, dass sich diejenigen der betrachteten kausalen Theorien, die Rückschlüsse auf kognitive Verarbeitungsprozesse ermöglichen, nicht einheitlich in der Zwei-Prozess-Debatte verorten lassen. Einige der Theorien lassen sich mit dem Wirken von Typ 1-Verarbeitungsprozessen vereinbaren, während andere von der Notwendigkeit einer Beteiligung von Typ 2-Prozessen ausgehen. Zudem gibt es Theorien, die annehmen, dass beide Arten von Prozessen an der Informationsverarbeitung beteiligt sind. Betrachtet man die in den verschiedenen Ansätzen verwendeten Aufgaben, so ergeben sich Hinweise auf eine Beziehung zu den angenommenen Verarbeitungsprozessen. Aufgaben in Theorien, bei denen Typ 2-Prozesse als notwendig erachtet werden, scheinen andere Anforderungen an die Versuchspersonen zu stellen als die Aufgaben in den Theorien, bei denen Typ 1-Prozesse als ausreichend erachtet werden können.

\section{Methodik}

In den vorangegangenen Abschnitten wurden sowohl Zwei-Prozess-Ansätze im Hinblick auf die Berücksichtigung kausaler Phänomene untersucht, als auch kausale Theorien bezüglich einer Einordnung in die Zwei-Prozess-Sichtweise betrachtet. Wie bereits erwähnt ergab sich ein uneinheitliches Bild bezüglich der kognitiven Prozesse, die bei der Bearbeitung von Aufgaben mit kausalem Hintergrund ablaufen. Die Analyse der verschiedenen theoretischen Ansätze ergab, dass wahrscheinlich mehrere verschiedene kognitive Verarbeitungsprozesse bei der Bearbeitung von kausalen Aufgaben ablaufen. Eine Betrachtung der in den Ansätzen untersuchten Aufgaben erbrachte überdies Hinweise darauf, dass die Art der ablaufenden Verarbeitungsprozesse von der Art der Aufgabe abhängt. Hieraus ergab sich die Forschungsfrage der vorliegenden Arbeit, die sich mit den Zusammenhängen zwischen unterschiedlichen kausalen Urteils- und Entscheidungsaufgaben und den zu Grunde liegenden kognitiven Prozessen beschäftigt. Zu diesem Zweck wurde eine 
vielfach verwendete experimentelle Methodik aus dem Bereich der Entscheidungsforschung (Dijksterhuis, 2004) für die Untersuchung kausaler Fragestellungen weiterentwickelt.

Im folgenden Abschnitt wird das methodische Vorgehen für die in den Abschnitten 4.2 bis 4.6 beschriebenen Experimente vorgestellt und erläutert. Die Grundidee der eingesetzten Methodik besteht darin, mögliche kognitive Verarbeitungsprozesse differenziell einzuschränken, um zu überprüfen ob hierdurch die Leistung bei der Bearbeitung von Aufgaben beeinträchtigt wird. Bei einer Verschlechterung der Performanz ist davon auszugehen, dass die eingeschränkten Verarbeitungsprozesse unter normalen Bedingungen für die Bearbeitung der Aufgabe relevant sind. Der Fokus der vorliegenden Arbeit liegt dabei auf der Rolle deliberativer, Typ 2-Prozesse.

Im weiteren Verlauf dieses Abschnittes wird zunächst die Auswahl der experimentellen Bedingungen diskutiert, die eine Untersuchung der kognitiven Verarbeitungsprozesse (im Sinne der Typ 1-/Typ 2-Unterscheidung) bei der Bearbeitung von Aufgaben auf der Grundlage zuvor erlernten Kausalwissens ermöglichen sollen. Dabei wird auch auf eine in letzter Zeit umfangreich diskutierte Theorie, die Unconscious Thought Theory (Dijksterhuis \& Nordgren, 2006) eingegangen, die von der Existenz unbewusster, aber zeitintensiver Denkprozesse ausgeht. Im Anschluss wird auf die Auswahl der Aufgabenarten eingegangen, von denen angenommen wird, dass sie mittels unterschiedlicher kognitiver Prozesse verarbeitet werden. Schließlich werden zwei Hypothesen formuliert, die mit den in dieser Arbeit vorgestellten Experimenten überprüft werden sollen.

\subsection{Wahl der experimentellen Bedingungen}

Eine zentrale Frage der im Rahmen dieser Arbeit vorgestellten empirischen Untersuchungen ist, ob Urteile und Entscheidungen auf der Basis von Wissen über komplexe kausale Systeme besser werden, wenn sie überlegt (also mittels Typ 2-Prozessen) getroffen werden oder ob es auch möglich ist, spontan (mittels Typ 1-Prozessen) eine gute Leistung zu erzielen. Für die Beantwortung dieser Frage muss zunächst sichergestellt werden, dass alle beteiligten Versuchspersonen über eine einheitliche Wissensbasis verfügen, die sie anschließend nutzen können, um die ihnen gestellten Aufgaben zu absolvieren. Daher wurde bei den durchgeführten Experimenten großer Wert auf die Vermittlung und Überprüfung kausalen Wissens gelegt. Um den Einfluss von Vorwissen (also Wissen, das die Versuchspersonen bereits vor Beginn der Experimente gesammelt hatten) weitestgehend auszuschließen, wurden die für die Experimente verwendeten Kausalsysteme anhand fiktiver 
Inhalte vorgestellt. Die Kausalsysteme weisen eine im Vergleich zu anderen Studien im Bereich des kausalen Urteilens und Entscheidens (z.B. Hagmayer \& Sloman, 2009) relativ große Komplexität auf und müssen zudem von den Versuchspersonen erlernt werden, um die interessierende Fragestellung untersuchen zu können. Daher wurden plausibel erscheinende Coverstories konstruiert, deren Inhalte trotz der verwendeten fiktiven Kausalsysteme möglichst konkret sein sollten. In den Coverstories wurden die verschiedenen Variablen des Kausalsystems, sowie die kausalen Beziehungen zwischen ihnen explizit dargestellt. In vier der fünf vorgestellten Studien geschah dies auch unter Zuhilfenahme graphischer Darstellungen der Kausalsysteme. Im Anschluss an die Vermittlung hatten die Versuchspersonen Multiple-Choice-Tests zu absolvieren um den erfolgreichen Erwerb des Kausalwissens zu überprüfen. Die Multiple-Choice-Fragen betrafen beispielsweise die Kausalbeziehungen zwischen den verschiedenen Variablen. Eine korrekte Beantwortung sämtlicher Multiple-Choice-Fragen stellte die Voraussetzung für die Teilnahme an den weiteren Abschnitten des jeweiligen Experimentes dar.

Wie bereits erwähnt, dient die durch die soeben geschilderten Maßnahmen erschaffene gemeinsame Wissensbasis als Ausgangspunkt für die Untersuchung der Frage wie dieses Wissen von verschiedenen Verarbeitungsprozessen für Urteile und Entscheidungen genutzt werden kann. Bei den untersuchten Verarbeitungsprozessen kann zwischen Typ 1- und Typ 2Prozessen unterschieden werden. Da automatisch und unbewusst ablaufende Typ 1-Prozesse nicht eliminiert werden können, sollen die nun vorzustellenden experimentellen Bedingungen eine Manipulation der zeitintensiven und bewusstes Nachdenken erfordernden Typ 2Verarbeitungsprozessen ermöglichen. Das Wirken von Typ 2-Prozessen kann verhindert werden, indem den Versuchspersonen keine Zeit zum bewussten Nachdenken über die zu bewältigende Aufgabe zur Verfügung gestellt wird (Dijksterhuis, 2004; Glöckner \& Witteman, 2010; Mamede Schmidt, Rikers, Splinter \& van Saase, 2010; Thompson, Prowse Turner und Pennycook, 2011). In ihrer Untersuchung intuitiver Typ 1-Prozesse gehen Mamede und Kollegen (Mamede, Schmidt \& Penaforte, 2008; Mamede et al., 2010) beispielsweise davon aus, dass Urteile, die unmittelbar nach der Stimuluspräsentation abgegeben werden auf automatisch ablaufenden Mustererkennungsprozessen basieren, die im Sinne der hier verwendeten Terminologie als Typ 1-Verarbeitungsprozesse bezeichnet werden können. Ähnliche Annahmen über schnelle, intuitive Urteile als Ergebnis von Typ 1Verarbeitungsprozessen machen Thompson et al. (2011) in ihrer Analyse metakognitiver Faktoren bei der Urteilsbildung auf der Grundlage von Typ 1- und Typ 2-Prozessen. Sowohl Mamede und Kollegen (2010) als auch Thompson und Kollegen (2011) nehmen dabei an, 
dass während der Informationsaufnahme lediglich Verarbeitungsprozesse vom Typ 1 ablaufen da die bewusste Aufmerksamkeit während der Stimuluspräsentation für die Aufnahme der Informationen benötigt wird. Insgesamt kann also davon ausgegangen werden, dass eine Antwort, die unmittelbar nach der Präsentation von Aufgabe und zugehörigen Stimuli abgegeben wird auf intuitiven Typ 1-Prozessen basiert.

Eine weitere Möglichkeit der Verhinderung von Typ 2-Prozessen besteht darin, das Arbeitsgedächtnis der Versuchspersonen zwischen Stimuluspräsentation und Abgabe der Antwort mit einer zusätzlichen Aufgabe auszulasten. Auf diese Weise kann überprüft werden, ob zeitintensive, aber unbewusst ablaufende kognitive Prozesse oder klassische Typ 2Prozesse für die Lösung der Aufgabe benötigt werden. Die Möglichkeit solcher langsam ablaufender, unbewusster Verarbeitungsprozesse wird beispielsweise bei Glöckner und Witteman (2010) unter dem Begriff der konstruktiven Intuition (constructive intuition) und bei Dijksterhuis \& Nordgren (2006; s. Abschnitt 3.2) als unbewusstes Denken erörtert.

Zur Untersuchung der Fragestellung der vorliegenden Arbeit sind somit drei verschiedene Arten von Verarbeitungsbedingungen erforderlich. Erstens soll ermöglicht werden, dass Versuchspersonen bewusst mittels Typ 2-Prozessen über die zu bearbeitende Aufgabe nachdenken können (Bedingung des bewussten Nachdenkens). Im Gegensatz dazu sollen in einer zweiten Bedingung spontane bzw. intuitive, auf Typ 1-Prozessen basierende, Entscheidungen abgegeben werden (Bedingung des spontanen Entscheidens). Drittens sollen Urteile erzeugt werden, bei denen den Versuchspersonen zwar Zeit zur Verfügung stand, sie diese aber nicht zum bewussten Denken nutzen konnten (Bedingung des unbewussten Denkens).

Neben der Manipulation der kognitiven Verarbeitungsprozesse mittels der verschiedenen experimentellen Bedingungen wurde in den in dieser Arbeit vorgestellten Experimenten durch weitere Maßnahmen Einfluss auf die Verarbeitung der präsentierten Informationen genommen. Zum einen wurde die Zeitspanne, für die die einzelnen Attributsausprägungen der präsentierten Fälle präsentiert wurden relativ kurz gewählt, um das Ausmaß der bewussten Verarbeitung während der Darbietung zu begrenzen. Es wurde jedoch durch Vortests sichergestellt, dass ausreichend Zeit zum bewussten Wahrnehmen der Stimuli zur Verfügung stand. Durch diese Veränderung wurde eine bessere Kontrolle der während der Stimuluspräsentation ablaufenden kognitiven Prozesse möglich als dies in anderen Studien der Fall war. In der Studie von Mamede et al. (2010) hatten die Versuchspersonen beispielsweise die Aufgabe, medizinische Fall-Vignetten zu lesen und im Anschluss die erste 
Diagnose, die ihnen in den Sinn kam, zu notieren. Da den Versuchspersonen keinerlei Restriktionen bezüglich der zur Verfügung stehenden Zeit gemacht wurden kann nicht ausgeschlossen werden, dass sie bereits während der Stimuluspräsentation mittels Typ 2Verarbeitungsprozessen über die Lösung der zu bearbeitenden Aufgabe nachgedacht haben. Ähnlich verhielt es sich in den Experimenten von Thompson et al. (2011). Auch hier wurde vom Wirken intuitiver Typ 1-Prozesse ausgegangen, obwohl den Versuchspersonen keinerlei Restriktionen bezüglich der Verarbeitung des verwendeten Stimulusmaterials auferlegt wurden und damit Typ-2-Verarbeitungsprozesse möglich waren. Des Weiteren wurden die Versuchspersonen in den im Rahmen dieser Arbeit berichteten Experimenten aufgefordert, ihr Urteil im Anschluss an die jeweilige Verarbeitungsbedingung so schnell wie möglich abzugeben. Dies sollte bewusstes Nachdenken unmittelbar vor Abgabe des Urteils verhindern, da dies die Ergebnisse, vor allem in den Bedingungen, in denen bewusstes Nachdenken verhindert werden sollte, verzerren würde.

Für die konkrete Ausgestaltung der verschiedenen Verarbeitungsbedingungen unter denen die ablaufenden Verarbeitungsprozesse untersucht werden sollten, wurde ein bestehendes experimentelles Paradigma verwendet und für die Bearbeitung von Aufgaben mit kausalem Hintergrund durch die oben erläuterten Maßnahmen erweitert. Hierbei handelt es sich um ein Paradigma, das von Dijksterhuis (2004) entwickelt und im Rahmen von Experimenten zur Untersuchung der Unconscious Thought Theory (UTT; Dijksterhuis \& Nordgren, 2006) bereits vielfach verwendet wurde. Zu Beginn der diesem Paradigma folgenden Experimente werden die Versuchspersonen über die später von ihnen zu lösende Aufgabe informiert. Diese Aufgabe besteht zumeist aus der Bewertung verschiedener Alternativen hinsichtlich eines Kriteriums oder der Auswahl der hinsichtlich des Kriteriums besten Alternative. Im Anschluss werden die Informationen präsentiert, die die Versuchspersonen für die Lösung der Aufgabe benötigen. Die Informationen bestehen in der Regel aus Beschreibungen der Eigenschaftsausprägungen der zu beurteilenden Alternativen. Dabei ist es wichtig, dass die Versuchspersonen Gelegenheit haben, die Informationen bewusst wahrzunehmen. Es wird jedoch davon ausgegangen, dass sie sie nicht in für die Bewältigung der Aufgabe nötigem Maße bewusst weiter verarbeiten können, da ihre Aufmerksamkeit durch die Informationsaufnahme gebunden wird (vgl. Dijksterhuis, 2004). Unmittelbar nach der Stimuluspräsentation erfolgt die experimentelle Manipulation. Ein Teil der Versuchspersonen wird aufgefordert, sofort ihr Urteil bezüglich der Aufgabe abzugeben. In dieser Bedingung spontanen bzw. sofortigen Entscheidens werden den Probanden somit intuitive, auf Typ 1-Prozessen basierende, Urteile abverlangt, da nicht genügend Zeit zum 
Ablauf von Typ 2-Prozessen zur Verfügung steht. Ein anderer Teil der Versuchspersonen wird aufgefordert, für einen festgelegten Zeitraum gründlich über die Aufgabe nachzudenken und im Anschluss ihr Urteil abzugeben. Diese Bedingung des bewussten Nachdenkens soll Entscheidungen hervorbringen, für die die Bedeutung der Informationen für das zu fällende Urteil intensiv mittels Typ 2-Prozessen evaluiert wird. Eine dritte Gruppe von Versuchspersonen soll für den gleichen Zeitraum eine Distraktoraufgabe absolvieren, die ihre volle Aufmerksamkeit erfordert. Im Anschluss sollen auch sie ihr Urteil hinsichtlich der ursprünglichen Aufgabe abgeben. In dieser Bedingung des unbewussten Denkens soll langsamen, unbewussten Denkprozessen, die dann ablaufen, wenn das zu lösende Problem außerhalb des Fokus der Aufmerksamkeit liegt, die Möglichkeit gegeben werden zu einem Urteil zu gelangen.

Das experimentelle Paradigma der UTT eignet sich gut, um die für diese Arbeit relevante Fragestellung $\mathrm{zu}$ untersuchen. Mit den vorgestellten experimentellen Manipulationen (spontanes Entscheiden, Entscheiden nach einer Phase unbewussten Denkens, Entscheiden nach einer Phase bewussten Denkens) kann getestet werden, welche Art von kognitiven Verarbeitungsprozessen Urteilen auf der Grundlage von Wissen über komplexe kausale System zu Grunde liegen. Im Sinne einer zwei-Prozess-Sichtweise sollen Entscheidungen, die unmittelbar nach der Stimuluspräsentation getroffen werden müssen (spontane Bedingung) der Untersuchung von Typ 1-Urteilsprozessen dienen, da den Versuchspersonen nicht genügend Zeit zur Verfügung steht, um ihre Entscheidung gründlich und bewusst zu überdenken. Entscheidungen, die nach einer Phase bewussten Nachdenkens über die zu lösende Aufgabe getroffen wurden, dienen hingegen der Untersuchung von Typ 2Verarbeitungsprozessen. Aus den oben genannten Gründen stehen Entscheidungen, die nach einer Phase unbewussten Denkens abgegeben werden, außerhalb der Typ 1-/Typ 2Dichotomie. Ihre Untersuchung soll zeigen, ob es möglicherweise Verarbeitungsprozesse im Bereich des kausalen Urteilens und Entscheidens gibt, die zwar längere Zeit in Anspruch nehmen, aber sonst ähnliche Charakteristika wie Typ 1-Verarbeitungsprozessen aufweisen.

Durch die Verwendung der vorgestellten experimentellen Bedingungen sind mehrere Ergebnismuster möglich, die unterschiedliche Interpretationen nach sich ziehen: Zeigen sich keine Unterschiede in der Qualität der Urteile zwischen den verschiedenen Bedingungen so beruht das Urteil auf Typ 1-Prozessen, da in der Bedingung spontanen Entscheidens nur diese Art von Prozessen ablaufen kann und das Urteil nicht durch anschließend ablaufende Prozesse modifiziert wird (vgl. Mamede et al., 2010; Thompson et al., 2011). Lassen sich die besten 
Leistungen hingegen in der Bedingung unbewussten Denkens feststellen, so basiert das Urteil auf intuitiven, aber langsam ablaufenden Prozessen. Die schlechtere Leistung in der Bedingung bewussten Denkens könnte im Sinne von Urteilsverzerrungen interpretiert werden (vgl. Dijksterhuis \& Nordgren, 2006). Führt die Bedingung bewussten Denkens zu den besten Leistungen, kann dies als Evidenz für eine Notwendigkeit bewusster Typ 2Verarbeitungsprozesse angesehen werden. Sollten die Bedingungen bewussten und unbewussten Denkens zu den besten Ergebnissen führen, so kann angenommen werden, dass das Urteil auf der Grundlage von langsam ablaufenden, unbewussten Prozessen gebildet wird, die nicht von Typ 2-Prozessen verzerrt werden. Liegt hingegen die beste Leistung in den Bedingungen sofortigen Entscheidens sowie bewussten Denkens vor, so wäre dies ein Beleg für ein auf Typ 1-Prozessen basierendes intuitives Urteil, das durch Typ 2-Prozesse überprüft und bestätigt wird, ohne dass es hierbei zu Verzerrungen kommt. Bei dieser Konstellation wäre des Weiteren davon auszugehen, dass die Blockierung des Arbeitsgedächtnisses in der Bedingung des unbewussten Denkens zu einer Einschränkung der Erinnerungsleistung in Bezug auf das spontan gebildete Urteil führt.

Da die Unconscious Thought Theory (Dijksterhuis \& Nordgren, 2006) neben dem experimentellen Paradigma auch im Hinblick auf ihre Aussagen über die Effektivität der verschiedenen Verarbeitungsbedingungen bei der Bearbeitung von komplexen Aufgaben für die vorliegende Arbeit interessant ist, wird sie im Folgenden näher beschrieben.

\subsection{Die Theorie des unbewussten Denkens (Dijksterhuis \& Nordgren, 2006)}

Dijksterhuis \& Nordgren (2006) stellten mit der Unconscious Thought Theory (UTT) die These auf, dass Entscheidungen nach einer Phase des sog. unbewussten Denkens unter bestimmten Umständen besser ausfallen als nach einer Phase bewussten Nachdenkens. Damit stellt sich die Theorie gegen die in der Forschung vorherrschende Ansicht, dass bewusstes Denken am besten geeignet ist, komplexe Entscheidungen zu treffen (vgl. Dijksterhuis, Bos, Nordgren \& van Baaren, 2006). Gleichzeitig entspricht sie jedoch der Volksweisheit, dass schwierige Entscheidungen leichter fallen und zu besseren Resultaten führen, wenn man eine Nacht über sie schläft. Dijksterhuis und Nordgren (2006) formulieren sechs Prinzipen, die die Grundlage ihrer Theorie bilden. Das erste Prinzip (Unconscious Thought Principle) besagt, dass es neben dem bewussten Denken auch unbewusste Denkprozesse gibt, die aktiv sind während die (bewusste) Aufmerksamkeit nicht auf dem zu lösenden Problem liegt. Der Fokus der Aufmerksamkeit wird als wesentliches Unterscheidungskriterium zwischen bewusstem und unbewusstem Denken angesehen. 
Das zweite Prinzip der UTT (Capacity Principle) besagt, dass sich bewusstes und unbewusstes Denken hinsichtlich ihrer Informationsverarbeitungskapazität deutlich voneinander unterscheiden. Während die Kapazität des Bewusstseins derjenigen des Arbeitsgedächtnisses entspricht (Dijksterhuis und Nordgren sprechen hier von bis zu 60 bits pro Sekunde) ist die Kapazität des Unbewussten nahezu unbegrenzt (etwa 11.200.000 bits pro Sekunde, von denen etwa 10.000.000 auf das visuelle System entfallen). Die Unterschiede in der Kapazität werden als Hauptgrund für die überlegene Leistung des unbewussten Denkens beim Lösen komplexer Probleme angesehen. Nach dem dritten Prinzip (Bottom-Up-VersusTop-Down Principle) unterscheiden sich die beiden Arten des Denkens auch über die Art der Informationsverarbeitung. Während das bewusste Denken im Sinne einer top-downInformationsverarbeitung operiert und dadurch anfällig für Verzerrungen wie Stereotypisierungen wird, arbeitet das unbewusste Denken im Sinne einer bottom-upVerarbeitung von Informationen und sollte daher nicht von Urteilsverzerrungen betroffen sein. Vielmehr führt der Prozess des unbewussten Denkens über eine langsame Integration der vorhandenen Informationen zu objektiven, zusammenfassenden Urteilen.

Dem vierten Prinzip (Weighting Principle) zufolge werden die bei einer Entscheidung relevanten Attribute automatisch auf Basis ihrer relativen Bedeutung für die Entscheidung gewichtet. Beim bewussten Denken werden die Attribute hingegen oftmals falsch gewichtet. Das fünfte Prinzip (Rule Principle) besagt, dass das bewusste Denken in der Lage ist, Regeln explizit und präzise zu befolgen. So können Antworten auf Rechenaufgaben mit bewusstem Denken gelöst werden. Unbewusstes Denken ist hierzu nicht in der Lage, da es eher in groben Schätzungen resultiert. Es kann Regeln nicht strikt folgen, sondern ihnen lediglich entsprechen. Nach dem sechsten und letzten Prinzip der UTT (Convergence-VersusDivergence Principle) ist bewusstes Denken fokussiert und strebt nach Konvergenz. Im Gegensatz erlaubt das unbewusste Denken mehr Divergenz was sich den Autoren zufolge vor allem in einem größeren Ausmaß kreativer und ungewöhnlicher Problemlösungen niederschlägt.

Die auf den geschilderten sechs Prinzipien beruhende UTT wurde in einer Vielzahl empirischer Studien mit dem von Dijksterhuis (2004) entwickelten experimentellen Paradigma getestet, das bereits im vorangegangenen Abschnitt beschrieben wurde. Folgendes Beispiel soll den Ablauf eines typischen Experiments zur UTT veranschaulichen: In einem Experiment von Dijksterhuis und Kollegen (2006) hatten die Versuchspersonen die Aufgabe, das beste von vier präsentierten Autos auszuwählen. Die Autos wurden durch verschiedene 
Attribute charakterisiert, die jeweils in einer als positiv und einer als negativ bewerteten Ausprägung vorlagen (z.B. hoher vs. niedriger Verbrauch, großer vs. kleiner Kofferraum). Über die Anzahl der postiven bzw. negativen Attributsausprägungen der verschiedenen Autos war eine Bewertung der Güte der verschiedenen Alternativen möglich. Nach der Präsentation der Attributsausprägungen für die vier Automodelle fand die oben erläuterte Manipulation der Verarbeitungsbedingungen (mit Ausnahme der Bedingung spontanen Entscheidens, die in diesem Experiment nicht erhoben wurde) statt. Die im Anschluss abgegebenen Urteile zeigten, dass bei Versuchspersonen, denen vier Attribute pro Auto präsentiert wurden die Bedingung des bewussten Nachdenkens zu den besten Ergebnissen führte. Wurden jedoch 12 Attribute pro Auto präsentiert, so änderte sich das Bild. Nun waren die Versuchspersonen in der Bedingung des unbewussten Denkens denen in der Bedingung des bewussten Denkens überlegen. Dieser Effekt wird vor allem auf die nachlassende Leistungsfähigkeit des bewussten Denkens bei komplexeren Aufgaben zurückgeführt, der auf die begrenzte Kapazität dieser Art der Informationsverarbeitung zurückgeführt wird (s. Capacity Principle). Da das unbewusste Denken dieser Restriktion nicht unterliegt, bleibt seine Leistungsfähigkeit bestehen und wird in Relation zum bewussten Denken somit bei zunehmender Komplexität der Aufgabe besser.

Die verschiedenen Experimente zur Überprüfung der auf Grundlage der UTT aufgestellten Thesen erbrachten jedoch keine einheitliche Befundlage. Während die Unterstützer der Theorie eine große Anzahl von Experimenten vorlegten, die die Gültigkeit der UTT in einer Vielzahl von Situationen und Entscheidungsarten belegen (z.B. Dijksterhuis, 2004; Bos \& Dijksterhuis, 2010) gibt es auch etliche Studien, die nicht in der Lage waren, die Überlegenheit des unbewussten Denkens zu replizieren (z.B. Newell, Wong, Cheung \& Rakow, 2009; Waroquier, Marchiori, Klein \& Cleeremans, 2010). Zwei Metaanalysen, die versuchen, die Ergebnisse der verschiedenen Studien dieser empirisch orientierten Debatte einzuordnen, kommen ebenfalls zu widersprüchlichen Schlussfolgerungen. Während eine der Analysen (Strick, Dijksterhuis, Bos, Sjoerdsma \& van Baaren, 2011) zu Ergebnissen kommt, die die Annahmen der UTT stützen, kann die andere (Acker, 2008) keine überzeugende Evidenz im Sinne der Theorie entdecken. Neben den empirischen Arbeiten, die versuchen zur Diskussion über die UTT beizutragen, gibt es inzwischen auch eine Reihe theoretischer Arbeiten, die sich kritisch mit den theoretischen Grundlagen des unbewussten Denkens auseinandersetzen (z.B. Bargh, 2011; Gonzáles-Vallejo, Lassiter, Bellezza \& Lindberg, 2008). Zuletzt sei noch eine aktuelle Ausgabe der Zeitschrift Social Cognition (Sherman, 2011) erwähnt, die sich ausschließlich dem aktuellen Stand der Debatte um die UTT widmet. 
Wie ersichtlich hat sich eine Kontroverse um die UTT entwickelt, die bislang nicht aufgelöst werden konnte.

Die Einordnung des unbewussten Denkens in die Zwei-Prozess-Debatte gestaltet sich auch vom Standpunkt der UTT aus als schwierig. Das unbewusste Denken verfügt zwar über eine Vielzahl von Eigenschaften, die klassischerweise Prozessen vom Typ 1 zugeordneten werden (z.B. unbewusster und automatischer Ablauf, hohe Kapazität, niedriger kognitiver Aufwand; vgl. Abschnitt 2.2.2), es wird aber zugleich als langsam charakterisiert und diese Eigenschaft findet sich typischerweise bei Typ 2-Verarbeitungsprozessen. Dijksterhuis und Nordgren (2006) weisen zudem auf weitere Probleme hin, die mit einer Einordnung unbewusster Denkprozesse in eine Zwei-System-Sichtweise verbunden sind. Darüber hinaus ist es unklar, inwiefern die verschiedenen Prinzipien der UTT Vorhersagen für den Bereich des kausalen Urteilens und Entscheidens zulassen. So kann beispielsweise nicht eindeutig geklärt werden, ob die Berücksichtigung kausaler Beziehungen, wie sie bei der Abschätzung von Interventionsfolgen nötig ist, in den Bereich des Folgens von Regeln oder dem Entsprechen von Regeln fällt. Ein anderes Beispiel betrifft die automatische Gewichtung von Attributen: Es ist unklar, ob dieses Prinzip auch auf den kausalen Status von Variablen in einem Kausalsystem anwendbar ist, der schließlich auch als eine Art von Gewicht verstanden werden kann. Wäre das Prinzip anwendbar, so wäre gemäß der UTT davon auszugehen, dass kausale Kategorisierungen von einer Phase unbewussten Denkens profitieren könnten.

Da die Frage nach den potenziellen Vorteilen unbewussten Denkens bei kausalen Kategorisierungs- und Interventionsaufgaben gut mit dem experimentellen Paradigma dieser Arbeit untersucht werden kann erscheint es sinnvoll, die eigene Fragestellung um die potenzielle Erweiterbarkeit der UTT auf das Gebiet des kausalen Urteilens und Entscheidens zu ergänzen. Ergebnisse, die einen Vorteil unbewussten Denkens gegenüber den anderen Verarbeitungsbedingungen belegen, würden für die Existenz einer dritten, langsamen und unbewussten Art der Informationsverarbeitung sprechen (vgl. Dijksterhuis \& Nordgren, 2006) und damit eine bedeutende Herausforderung für die im Fokus dieser Arbeit stehende ZweiProzess-Debatte darstellen.

\subsection{Auswahl der Aufgabenarten}

Nachdem im Abschnitt 3.1 erläutert wurde, wie die Verarbeitung der Informationen kontrolliert werden soll, um die zu Grunde liegenden Verarbeitungsprozesse zu analysiseren, wird nun die Auswahl der in den Experimenten eingesetzten Aufgaben diskutiert. Da 
unterschiedliche Aufgabenarten wahrscheinlich anhand unterschiedlicher Prozesse verarbeitet werden, sollten Aufgaben gewählt werden, die möglichst verschiedenartige Anforderungen an die Verarbeitung von Informationen vor dem Hintergrund kausalen Vorwissens stellen. Kausale Kategorisierungen und Interventionen in Kausalsysteme stellen zwei Aufgabenarten dar, die diesem Kriterium genügen.

Ansätze zu kausalen Kategorisierungen (z.B. Ahn et al., 2000; Rehder \& Kim, 2010) wurden in Abschnitt 2.4.5.3 als agnostisch hinsichtlich der Typ 1- /Typ 2-ProzessUnterscheidung klassifiziert, weil diese Ansätze keine expliziten Aussagen über die zu Grunde liegenden kognitiven Prozesse machen. Diese Klassifikation steht im Einklang mit dem uneinheitlichen Umgang mit Kategorisierungen bei den in Abschnitt 2.3 vorgestellten Zwei-Prozess-Theorien. Während Kahneman und Frederick (2002), sowie Smith und DeCoster (2000) davon ausgehen, dass Kategorisierungen auch mittels Typ 1Verarbeitungsprozessen vorgenommen werden, nehmen Sloman (1996), sowie Strack und Deutsch (2004) an, dass Typ 2-Prozesse notwendig für die Durchführung von Kategorisierungen sind.

Empirische Belege für mittels Typ 1-Verarbeitungsprozessen vorgenommene Kategorisierungen auf der Grundlage des kausalen Status der beteiligten Variablen erbrachten Luhmann et al. (2006; s. Abschnitt 2.4.2.3). Darüber hinaus entsteht beispielsweise bei der Betrachtung der causal model theoy of categorization (Rehder, 2003) der Eindruck, dass die komplexen Modelle, mit denen kausale Kategorisierungsvorgänge modelliert werden nur schwer mit bewussten kognitiven (Typ 2-) Prozessen in Einklang gebracht werden können. So ist es beispielsweise kaum vorstellbar, dass die Berechnung Bayesianischer Likelihoods, die im Modell von Rehder Kategorisierungsurteile von Versuchspersonen vorhersagen, überhaupt bewusst zu bewältigen sind. Die Aussage von Rehder und Kim (2010), intuitive Kategorisierungsurteile zu untersuchen, stellt zudem einen Hinweis auf eine Verarbeitung mittels Typ 1-Prozessen dar. Da sie kausale Kohärenz als wesentlichen Faktor bei der kausalen Kategorisierung ansehen, liegt es nahe anzunehmen, dass dieser Effekt auf Typ 1Prozessen beruht. Natürlich sind Modellannahmen nicht mit Annahmen über psychologische Prozesse gleichzusetzen. Würden Kategorisierungsentscheidungen jedoch ausschließlich durch bewusstes Nachdenken getroffen werden, so erscheint es unwahrscheinlich, dass es der sehr aufwändigen (und nicht ohne Rechenhilfen durchführbaren) Berechnung bedingter Wahrscheinlichkeiten bedarf, um ein Urteil zu bilden. Weitere Hinweise für intuitive Typ 1Prozesse bei der Durchführung von Kategorisierungen finden sich in Exemplar-basierten 
Ansätzen (z.B. Juslin, Olsson \& Olsson, 2003; Nosofsky, 1986). Juslin et al. (2003) charakterisieren die mit Exemplar-Modellen verbundenen kognitiven Verarbeitungsprozesse als automatisch, schnell und ähnlichkeitsbasiert. Sie können somit als Typ 1-Prozesse verstanden werden.

Insgesamt besteht somit Grund für die Annahme, dass Kategorisierungen auch unter Bedingungen, in denen ein spontanes, auf Typ 1-Verarbeitungsprozessen basierendes Urteil erforderlich bzw. sinnvoll ist, erfolgreich vorgenommen werden können. Daher dienen sie in den im Rahmen dieser Arbeit durchgeführten Experimenten als eine Art von Aufgaben, die auf der Basis kausalen Vorwissens von den Versuchspersonen bearbeitet werden sollen.

Die Wahl von Interventionsaufgaben als zweite zu untersuchende Aufgabenart wird im Folgenden erläutert. In der Causal Model Theory of Choice (Sloman \& Hagmayer, 2006; Hagmayer \& Sloman, 2009; s. Abschnitt 2.4.3.3) wird detailliert beschrieben, wie Menschen bei der Bewertung verschiedener Interventionsmöglichkeiten vorgehen. Im Wesentlichen simulieren sie dabei die Wirkungen, die die zur Wahl stehenden Interventionen in ein Kausalsystem auf Grundlage der kausalen Beziehungen dieses Systems haben würden. Im Anschluss wird diejenige Intervention ausgewählt, die die für die erfolgreiche Bewältigung der zu bearbeitenden Aufgabe günstigsten Wirkungen nach sich zieht. Da die Abschätzung der Interventionsfolgen nur mittels mentaler Simulationen bewerkstelligt werden kann und diese offensichtlich bewusstes (Typ 2-) Denken erfordern (vgl. Lagnado, 2011; Abschnitt 2.4.3.2) erscheinen Interventionsaufgaben für die im Rahmen dieser Arbeit interessierende Fragestellung überaus geeignet. Sie werden daher in den vorzustellenden Experimenten verwendet.

Die Rolle mentaler Simulationen bei der Urteilsbildung wurde mit einer dritten Aufgabenart untersucht. Im Gegensatz zu den anderen Experimenten sollten die Teilnehmer nicht über Interventionen entscheiden, sondern Situationen beurteilen, die sich kausal weiterentwickeln. Zugleich soll geprüft werden, ob die in Abschnitt 3.2 vorgestellten Unconscious Thought Theory (Dijksterhuis \& Nordgren, 2006) für den Bereich des kausalen Urteilens und Entscheidens gültig ist. Da die UTT davon ausgeht, dass die Vorteile des unbewussten Denkens gegenüber dem bewussten Denken mit steigender Komplexität der Aufgabe größer werden, wurde die Aufgabe so konstruiert, dass die Versuchspersonen eine relativ große Anzahl an Informationen zu verarbeiten hatten. Für eine Testung der Theorie vor dem Hintergrund kausalen Vorwissens wurde daher eine Aufgabe entwickelt, in der entsprechend typischer Experimente zur UTT mehrere Alternativen in einem Zustand vor dem 
Wirken der instruierten kausalen Mechanismen präsentiert werden. Die Aufgabe der Versuchsperson besteht darin, die Alternativen hinsichtlich eines einfachen Kriteriums nach Wirken der kausalen Mechanismen zu bewerten. Die Grundlage der Bewertung bilden mentale Simulationen des kausalen Einflusses in den Alternativen.

\subsection{Hypothesen}

Für die gewählten Verarbeitungsbedingungen und Aufgabenarten werden auf Grundlage der Erläuterungen im vorangegangenen Abschnitt folgende Hypothesen aufgestellt:

1. Kausale Kategorisierungsurteile können auf Grundlage von Typ 1Verarbeitungsprozessen getroffen werden.

Erläuterung Hypothese 1: Bei Kategorisierungsurteilen stehen Typ 1Verarbeitungsprozesse im Vordergrund. Es sollten sich daher keine Vorteile bewussten oder unbewussten Nachdenkens gegenüber spontanem Entscheiden finden lassen. Es wird davon ausgegangen, dass Kategorisierungsurteile sowohl durch die Anzahl der für eine bestimmte Kategorie charakteristischen Variablenausprägungen (vgl. Rosch \& Mervis, 1975) als auch durch kausale Faktoren wie die in Abschnitt 2.4.5.3 beschriebenen kausalen Staus- und Kohärenzeffekte (Ahn et al., 2000; Rehder \& Kim, 2010) bestimmt werden. Die Stärke der kausalen Faktoren bestimmt dabei deren Einfluss auf das Kategorisierungsurteil. Je höher der kausale Status einer Variablen, desto größer sollte ihr Einfluss auf das Kategorisierungsurteil sein.

2. Bei Interventionsurteilen ist die Beteiligung von Typ 2-Verarbeitungsprozessen notwendig.

Erläuterung Hypothese 2: Wie die Causal Model Theory of Choice (Sloman \& Hagmayer, 2006; Hagmayer \& Sloman, 2009) annimmt, werden bei Interventionsentscheidungen Typ 2-Prozesse genutzt. Daher sollte bewusstes Nachdenken die Performanz bei Interventionsurteilen im Vergleich zum spontanen Entscheiden verbessern. Interventionsurteile werden primär durch die Anzahl der von den zur Wahl stehenden Interventionen beeinflussbaren Variablen bestimmt (vgl.Hagmayer \& Sloman, 2009). Diese Zahl wird in mentalen Simulationsprozessen evaluiert, die die kausalen Konsequenzen der zur Auswahl stehenden Interventionen auf Grundlage von Wissen über das Kausalsystem, in das interveniert werden soll, vorhersagen. Der kausale Status der beeinflussbaren Variablen 
beeinflusst die Urteile möglicherweise ebenfalls (vgl. Proctor \& Ahn, 2007). Je höher der kausale Status einer Variablen, die durch die Intervention beeinflusst werden kann, desto größer ist ihr Einfluss auf das Interventionsurteil.

Aufgrund der unsicheren Befundlage zur UTT, den unklaren Vorhersagen, die diese Theorie für den Bereich des kausalen Urteilens und Entscheidens macht, sowie dem Umstand, dass sie bislang nicht auf diesem Gebiet getestet wurde, werden keine Hypothesen zum Verhältnis des unbewussten Denkens zu den anderen Verarbeitungsbedingungen gemacht. Die Untersuchung der Bedingung unbewussten Denkens hat damit einen exploratorischen Charakter. Ließe sich die UTT auch im Bereich des kausalen Urteilens und Entscheidens anwenden, so wäre aufgrund der in Abschnitt 3.2 aufgestellten Überlegungen jedoch zu erwarten, dass unbewusstes Denken zumindest bei Kategorisierungsurteilen zu guten Leistungen führt.

\section{Experimente}

\section{1 Überblick über die durchgeführten Experimente und allgemeiner Ablauf}

An dieser Stelle soll kurz auf die 5 Experimente eingegangen werden, in denen Verarbeitungsbedingungen, Kausalstrukturen und Aufgaben variiert wurden, um die im vorangegangenen Abschnitt aufgestellten Hypothesen zu prüfen. In den Experimenten 1 und 2 wurden mit gleichem Material sowohl Kategorisierungs- als auch Interventionsaufgaben untersucht. Experiment 3 beschäftigt sich näher mit den kognitiven Prozessen bei Kategorisierungsaufgaben, Experiment 4 mit denen bei Interventionsaufgaben. In Experiment 5 werden schließlich mentale Simulationen ohne Interventionen untersucht. In allen vorgestellten Experimenten wird die Bedingung spontanen Entscheidens, die Bedingung unbewussten Denkens, sowie mindestens eine Bedingung, die bewusstes Nachdenken ermöglicht, untersucht.

Im Folgenden wird der allgemeine Ablauf der vorgestellten Experimente ausführlich dargestellt. Da die Experimente umfangreiche Gemeinsamkeiten aufweisen erschien es sinnvoll, diesen Abschnitt der Vorstellung der eigentlichen Experimente voranzustellen. Im Anschluss werden dann die fünf für diese Arbeit durchgeführten Experimente nach jeweils dem gleichen Schema vorgestellt. Zunächst werden die Ziele des jeweiligen Experiments definiert und die Mittel skizziert, mit denen sie erreicht werden sollen. Danach werden die jeweilige Probandenstichprobe und das experimentelle Design vorgestellt. Im darauf 
folgenden umfangreicheren Abschnitt wird der Ablauf des jeweiligen Experimentes konkretisiert und das verwendete Stimulusmaterial vorgestellt. Dabei werden die den Versuchspersonen vermittelten Instruktionen bezüglich des jeweils verwendeten Kausalsystems im Wortlaut wiedergegeben. Zudem werden die jeweiligen Testfälle vorgestellt und Vorhersagen auf der Basis des instruierten Kausalsystems abgeleitet. Im Anschluss werden die Ergebnisse der Experimente vorgestellt und analysiert. Schließlich findet eine kurze Diskussion der jeweiligen Befunde statt. Aufgrund der Komplexität der Ergebnismuster wird die Diskussion von Befunden, die mehrere Experimente betreffen bzw. nicht mit den primären Hypothesen in Zusammenhang stehen in die Allgemeine Diskussion (Abschnitt 5) ausgelagert.

Die im Rahmen dieser Arbeit vorgestellten Experimente weisen trotz ihrer relativ hohen Komplexität eine Reihe von Gemeinsamkeiten auf. Diese sollen im Folgenden beschrieben werden, bevor auf die einzelnen Experimente detailliert eingegangen wird. Alle Experimente wurden an Computern im Georg-Elias-Müller-Institut für Psychologie der Universität Göttingen durchgeführt und wurden mit Hilfe von Visual Basic for Application mit Microsoft PowerPoint (Versionen 2007 und 2010) programmiert. Die Versuchspersonen wurden über ein für die Abteilung für Kognitionswissenschaften und Entscheidungspsychologie entwickeltes Datenbankprogramm rekrutiert. In Abhängigkeit ihrer individuellen zeitlichen Präferenzen wurden unter Berücksichtigung etwaiger Ausschlusskriterien Kombinationen von Experimenten erstellt, die die jeweilige Versuchsperson während eines Termins im Versuchslabor der Abteilung absolvierte. Daher waren die hier vorgestellten Experimente in der Regel Teil einer Abfolge verschiedener Studien. Die Versuchspersonen wurden für die Teilnahme an den Experimenten entweder mit $7 €$ pro Stunde entlohnt oder bekamen die abgeleistete Zeit als Teil einer im Rahmen ihres Studiums zu erbringenden Prüfungsleistung bescheinigt.

$\mathrm{Zu}$ Beginn eines jeden Experimentes wurden die Versuchspersonen mit der experimentellen Prozedur vertraut gemacht (vgl. Abbildung 2). Dies geschah, in dem sie Übungsaufgaben absolvierten, die auf die der Versuchsperson zugewiesene experimentelle Bedingung zugeschnitten waren. Insgesamt gab es vier verschiedene experimentelle Bedingungen (spontanes Entscheiden, Entscheiden nach einer Phase unbewussten Denkens, Entscheiden nach einer Phase bewussten Denkens, Entscheiden nach einer Phase bewussten Denkens mit selbst gewählter Dauer) und zwei verschiedene Aufgabentypen (Kategorisierung und Intervention), die in den vorgestellten Experimenten wiederholt durchgeführt wurden. 
Wie oben erläutert, dienten die verschiedenen Bedingungen der Untersuchung unterschiedlicher kognitiver Verarbeitungsprozesse. Im Folgenden wird der Ablauf der unterschiedlichen Bedingungen detailliert dargestellt. Dabei wird sowohl auf die experimentelle Prozedur als auch auf die den Versuchspersonen vermittelten Informationen eingegangen.

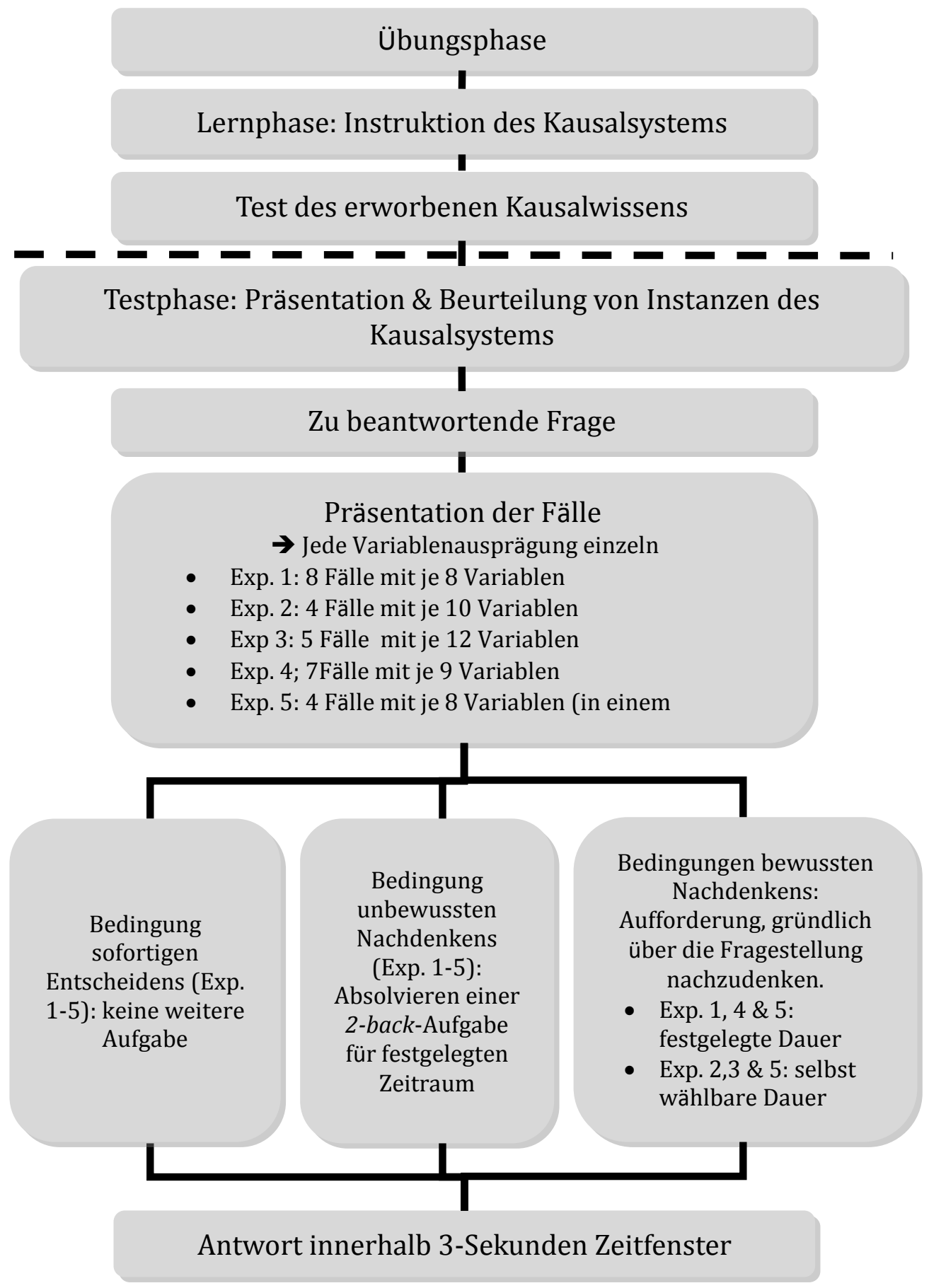

Abbildung 2: Schematische Darstellung des Ablaufs der vorgestellten Experimente. Weitere Erläuterungen im Text 
Zunächst wurden die Versuchspersonen darüber aufgeklärt, dass im nun folgenden Experiment untersucht werden soll, wie Menschen Entscheidungen in komplexen Kausalsystemen treffen, die zuvor von ihnen gelernt wurden. Anschließend wurden sie über die Möglichkeiten der Navigation zwischen den verschiedenen Folien des Experimentes aufgeklärt. Zudem wurden sie aufgefordert, die Instruktionen stets aufmerksam zu lesen und sich möglichst genau an sie zu halten. Während Übungs- und Lernphase waren auf den Folien Schaltflächen mit der Beschriftung „Zurück“ bzw. „Weiter“ eingeblendet, mittels derer die Versuchspersonen auf die vorangegangene bzw. die nächste Folie wechseln konnten. In Situationen, in denen dies nicht möglich war (z.B. auf der ersten bzw. letzten Folie einer Phase des Experiments), war die entsprechende Schaltfläche deaktiviert. Im Anschluss wurden die Versuchspersonen über die verschiedenen Phasen des Experimentes instruiert. Die in dieser Arbeit vorgestellten Experimente beinhalteten jeweils eine Übungsphase, die dazu dient, die Versuchspersonen mit der Art der Aufgaben des eigentlichen Experimentes vertraut $\mathrm{zu}$ machen. In der darauf folgenden Lernphase wurde den Versuchspersonen das Kausalsystem des jeweiligen Experimentes instruiert. Das erfolgreiche Lernen des Systems wurde mittels eines Multiple-Choice-Wissenstests am Ende der Lernphase überprüft. In der abschließenden Testphase hatten die Versuchspersonen bestimmte Aufgaben zu erfüllen, die mit dem gelernten System in Verbindung standen.

Die Übungsphase begann mit einfachen Einschätzungsaufgaben, in denen einfache Fragen innerhalb eines 3-sekündigen Zeitfensters beantwortet werden mussten. Den Versuchspersonen wurde mitgeteilt, dass der Zweck dieser Übungsaufgabe darin besteht, sie mit der Einschätzungsaufgabe und dem zur Verfügung stehenden Zeitfenster vertraut zu machen. Zudem wurden sie über die Struktur der Einschätzungsaufgabe informiert: Zunächst wurde für ca. 3 Sekunden eine Frage präsentiert. Im Anschluss wurde für kurze Zeit ein Bild gezeigt, für das gemäß der Frage eine Einschätzung abgegeben werden sollte. Unmittelbar nach der Präsentation des Bildes erschien eine elfstufige Skala, auf der sie innerhalb von 3 Sekunden ihre subjektive und spontane Einschätzung mittels Mausklick auf die entsprechende Schaltfläche abgeben sollen. Die Versuchspersonen wurden außerdem darüber in Kenntnis gesetzt, dass bei Überschreiten der 3-sekündigen Antwortfrist ein entsprechendes Hinweisfenster erscheint und dass sie die Übung erfolgreich beendet haben, sobald sie mehrere aufeinanderfolgender Fragen innerhalb des Zeitfensters beantworten konnten. Das Hinweisfenster wurde durch Anklicken einer mit „OK“ beschrifteten Schaltfläche wieder ausgeblendet, woraufhin das jeweilige Experiment automatisch weiterlief. Dieser Vorgang war beim Erscheinen sämtlicher Hinweisfenster in den vorgestellten Experimenten identisch 
und wird daher im Folgenden nicht mehr erwähnt. Die in den verschiedenen Übungsaufgaben verwendeten Fragen erforderten allesamt einfache Einschätzungen aus dem studentischen Umfeld. Eine während der Übungen verwendete Frage lautete beispielsweise „In welchen Vorlesungen lernen Studierende mehr, denen am Vormittag oder denen am Nachmittag?“. Die Pole der elfstufigen Antwortskale waren für diese Frage mit „sicher Vormittag“ bzw. „,sicher Nachmittag“ beschriftet, während die Antwortschaltflächen mit Werten von 0 bis 100 (Exp. 1) bzw. -5 bis +5 (Exp. 2-5) beschriftet waren.

Im Anschluss an diese Aufgabe folgten weitere, bedingungsabhängige Übungen. Einzig die Versuchspersonen in der spontanen Bedingung hatten keine weiteren Übungen zu absolvieren, da die bereits geschilderte Übung für diese Bedingungen bereits eine ausreichende Vorbereitung für die später erfolgende Testphase darstellte. Für alle anderen Bedingungen war jedoch jeweils eine weitere Übung notwendig, da hier in der Testphase eine bestimmte Aufgabe zwischen Stimuluspräsentation und Einschätzung zu absolvieren war. In der Bedingung des unbewussten Denkens bestand diese Aufgabe in der Bearbeitung einer sog. 2-back-Aufgabe, die eine Spezifizierung der sog. n-back-Aufgabe (vgl. Jonides et al., 1997) darstellt. Diese Aufgabe lastet das Arbeitsgedächtnis der Versuchspersonen zuverlässig aus und kann daher verhindern, dass während der Bearbeitung bewusst an die Einschäzung der zuvor präsentierten Simuli gedacht wird. Die n-back-Aufgabe wurde auch in mehreren Studien zur Unconscious Thought Theory als Distraktoraufgabe genutzt und in einer MetaAnalyse für effektiv befunden (Strick et al., 2011). Bei der 2-back-Aufgabe wird eine einstellige Zahl zwischen 1 und 9 auf dem Bildschirm präsentiert und die Versuchsperson hat die Aufgabe zu entscheiden, ob die präsentierte Zahl identisch mit der Zahl ist, die zwei Durchgänge zuvor präsentiert wurde. Die Aufgabe erfordert somit sowohl die kontinuierliche Repräsentation der letzten beiden präsentierten Zahlen als auch den Abgleich der vorletzten präsentierten Zahl mit der aktuell auf dem Bildschirm befindlichen Zahl. In den hier dargestellten Experimenten hatten die Versuchspersonen ihre Entscheidung mittels Mausklick auf eine von zwei Schaltflächen (beschriftet mit ,ja“ und „nein“) mitzuteilen. Unmittelbar danach wurde die nächste Zahl präsentiert. In den ersten beiden Durchgängen, in denen die Versuchspersonen noch keine Möglichkeit hatten, die präsentierte Zahl bezüglich der Übereinstimmung mit einer zwei Durchgänge zuvor präsentierten Zahl zu beurteilen, waren die beiden Antwortschaltflächen jeweils mit „,weiter“ beschriftet. Die für die 2-back-Aufgabe verbleibende Zeit wurde mittels eines Countdowns ebenfalls auf dem Bildschirm präsentiert. In den Instruktionen zur 2-back-Aufgabe wurden die Versuchspersonen aufgefordert, möglichst viele und möglichst genaue Antworten zu geben und sich bis zum Ende der 
Aufgabe voll zu konzentrieren. Zudem wurden sie darüber in Kenntnis gesetzt, dass unmittelbar vor Beginn der 2-back-Aufgabe wie in der vorherigen Übungsaufgabe für 3 Sekunden eine Frage präsentiert wird, die unmittelbar nach Ablauf der für die 2-backAufgabe vorgesehenen Zeit innerhalb des 3-Sekündigen Antwortfensters beantwortet werden sollte. Die Beantwortung der Frage stellte abermals eine Einschätzung dar, die mittels Mausklick auf eine von elf Schaltflächen abgegeben wurde. Der Inhalt der Frage war mit dem der vorangegangenen Übungsaufgabe vergleichbar. Die Versuchspersonen wurden darüber informiert, dass bei Überschreiten der 3-Sekündigen Antwortphase ein Hinweisfenster erscheint, in dem sie aufgefordert werden, beim nächsten Mal schneller zu antworten. Nach einmaligem Durchlaufen der Übungsaufgabe (unabhängig von der für die Antwort benötigten Zeit) war die Übungsphase abgeschlossen und die Versuchspersonen gelangten automatisch zum nächsten Teil des Experiments.

In den beiden Bedingungen des bewussten Nachdenkens wurde an Stelle der 2-backAufgabe auf dem Bildschirm die Aufforderung eingeblendet, gründlich über die bevorstehende Entscheidung nachzudenken. Der Unterschied zwischen den beiden Bedingungen bewussten Nachdenkens bestand in der Dauer der Phase des Nachdenkens. In der Bedingung mit festgelegter Dauer entsprach diese der Dauer der 2-back-Aufgabe in der Bedingung des unbewussten Denkens des jeweiligen Experimentes. In der Bedingung des bewussten Nachdenkens mit selbstgewählter Dauer konnten die Versuchspersonen hingegen selbst entscheiden, wie lange sie über die Entscheidung nachdenken wollten. Waren sie so weit, ihre Einschätzung abzugeben, so konnten sie auf eine mit „Weiter“ beschriftete Schaltfläche klicken (die in den Experimenten 2 und 3 jedoch erst nach Ablauf von 10 Sekunden anklickbar war). Ebenso wie in der Bedingung des unbewussten Denkens ging der Phase des Nachdenkens eine 3-sekündige Präsentation einer Frage unmittelbar voraus, bezüglich derer unmittelbar im Anschluss an die Phase des bewussten Nachdenkens eine Einschätzung abgegeben werden sollte. Dies geschah analog zu den anderen Übungsaufgaben mittels Mausklick auf eine von elf eine Skala darstellenden Schaltflächen in einem 3sekündigem Antwortfenster. Benötigte eine Versuchsperson länger als drei Sekunden um ihre Einschätzung abzugeben, so erschien auch in diesen Bedingungen ein Hinweisfenster mit der Aufforderung, beim nächsten Mal schneller zu antworten. Der Inhalt der Frage entsprach in beiden Bedingungen bewussten Nachdenkens wiederrum dem in der ersten Übungsaufgabe. Nach einem Durchgang dieser Übungsaufgabe war die Übungsphase abgeschlossen und die Versuchspersonen gelangten automatisch zum nächsten Teil des Experimentes. 
Nachdem die Versuchspersonen mit der Struktur der in der späteren Testphase zu bearbeitenden Aufgaben vertraut gemacht wurden, sollten sie in der nun folgenden Lernphase das im jeweiligen Experiment verwendete Kausalsystem erlernen. Zu diesem Zweck wurde ihnen mittels mehrerer Folien im Rahmen einer Coverstory die Struktur des Systems präsentiert. Dabei wurden die verwendeten Variablen sowie die zwischen ihnen bestehenden Beziehungen dargestellt. Den Versuchspersonen wurden keine zeitlichen Einschränkungen beim Erlernen des Kausalsystems auferlegt. Zudem konnten sie zwischen den verschiedenen das System betreffenden Instruktionsfolien nach Belieben hin und her wechseln. Auf der letzte Folie der Lernphase wurde den Versuchspersonen mitgeteilt, dass sie nun einen Multiple-Choice-Test zu absolvieren hätten, durch den sichergestellt werden soll, dass sie die verschiedenen Variablen und die Zusammenhänge zwischen ihnen richtig verstanden haben. Ferner wurden sie über die Anzahl der in diesem Test zu beantwortenden Fragen informiert und darüber in Kenntnis gesetzt, dass es für jede Frage genau eine richtige Antwort gibt. Es wurde ihnen mitgeteilt, dass sie während des Tests zwischen den einzelnen Fragen hin- und herspringen konnten und sie auf der Folie mit der letzten Frage auf die Schaltfläche mit der Beschriftung „Zur Auswertung“ klicken sollten, wenn sie alle Fragen beantwortet hatten. Unmittelbar danach würde ein Hinweisfenster erscheinen, dass die Versuchsperson darüber informiert, wie viele der Fragen sie richtig beantwortet hat. Sollten nicht alle Fragen korrekt beantwortet worden sein, so würden die Instruktionen des Kausalsystems erneut präsentiert. Für diesen Fall wurden die Versuchspersonen gebeten, diese nochmals aufmerksam durchzulesen und einzuprägen. Die Versuchspersonen hatten auf dieser Folie letztmalig vor dem Test die Möglichkeit, auf die Folien für die Instruktion des Kausalsystems zu wechseln. Während der Präsentation der Testfragen war diese Möglichkeit nicht mehr gegeben. Wenn sie bereit waren, den Test zu absolvieren sollten sie auf eine mit „Zum Test“ beschriftete Schaltfläche klicken.

Im nun folgenden Multiple-Choice-Test wurde den Versuchspersonen in zufälliger Reihenfolge eine vom jeweiligen Experiment abhängige Anzahl von Fragen präsentiert. Zusammen mit den Fragen wurden mehrere Antwortmöglichkeiten dargestellt, von denen eine mittels Anklicken einer vor der Antwort befindlichen Auswahlschaltfläche ausgewählt werden konnte. Die Versuchspersonen konnten sich durch Anklicken einer anderen Auswahlschaltfläche jederzeit für eine andere als die zuerst von ihnen ausgewählte Antwortalternative entscheiden. In diesem Falle wurde die zuerst getroffene Auswahlschaltfläche wieder in einen neutralen Zustand versetzt und die Schaltfläche der neu gewählten Antwort als aktiv markiert. Die Markierung einer einmal gewählten 
Antwortalternative für eine Frage wurde auch beim Wechsel zwischen den verschiedenen Fragen beibehalten. Während des Multiple-Choice-Tests gab es keine Einschränkungen bezüglich der den Versuchspersonen zur Verfügung stehenden Zeit. Hatte die Versuchsperson alle Fragen korrekt beantwortet, so gelangte sie in die Testphase des Experiments. War dies nicht der Fall, so wurden die Instruktionen des Kausalsystems erneut präsentiert und der Multiple-Choice-Test musste erneut absolviert werden. Gelang es einer Versuchsperson nicht, nach sechs Durchgängen alle Fragen des Tests korrekt zu beantworten gelangte sie zwar in die Testphase, ihr Datensatz wurde aber ohne ihr Wissen als ungültig markiert. Der Grund hierfür liegt zum einen in der Begrenzung des zeitlichen Aufwandes für die Durchführung des Experimentes. Des Weiteren sollte hierdurch sichergestellt werden, dass nur die Daten von denjenigen Versuchspersonen in die Analyse aufgenommen werden, die das Kausalsystem tatsächlich verstanden haben. Die Versuchspersonen, deren Daten als ungültig markiert wurden, wurden darüber nicht in Kenntnis gesetzt, um sie für eventuell im Anschluss durchgeführte Experimente nicht zu demotivieren.

Die anschließende Testphase war mit den Übungsaufgaben vergleichbar. Den Versuchspersonen wurde vor jedem Testdurchgang ein Bildschirm mit Instruktionen bezüglich des Ablaufs der nun folgenden Aufgabe präsentiert. Es wurde ebenfalls angezeigt, in welchem Versuchsdurchgang sich die Versuchsperson gerade befand und wie viele Durchgänge sie insgesamt zu absolvieren hatte. Die Anzahl der Durchgänge variierte von Experiment zu Experiment, der Ablauf war jedoch weitestgehend identisch (eventuelle Abweichungen werden bei der Beschreibung der einzelnen Experimente geschildert). Den Versuchspersonen wurde mitgeteilt, dass zunächst für ca. 3 Sekunden eine Frage präsentiert wird, die auf Grundlage der unmittelbar im Anschluss präsentierten Stimuli beantwortet werden sollte. Dabei wurde die zu beantwortende Frage bereits während der Instruktion explizit dargestellt. Danach würden bestimmte Ausprägungen der einzelnen Variablen des erlernten Kausalsystems einzeln für kurze Zeit präsentiert. Im Anschluss wurde der weitere, bedingungsabhängige weitere Ablauf dargestellt. In der Bedingung spontanen Entscheidens wurde den Versuchspersonen mitgeteilt, dass sie unmittelbar nach der Stimuluspräsentation ihre Einschätzung bezüglich der gestellten Frage abgeben sollten. In der Bedingung unbewussten Denkens hatten die Versuchspersonen vor der Beantwortung der Frage die oben beschriebene 2-back-Aufgabe für eine festgelegte Dauer zu absolvieren. In der Bedingung bewussten Nachdenkens mit festgelegter Dauer wurden die Versuchspersonen vor Beantwortung der Frage aufgefordert, für die gleiche Zeitspanne bewusst über ihre Antwort nachzudenken. Die Bedingung bewussten Nachdenkens mit selbstgewählter Dauer verlief mit 
einer Ausnahme identisch: Die Versuchspersonen konnten selbst entscheiden, zu welchem Zeitpunkt sie in ausreichendem Maße über die Beantwortung der Frage nachgedacht hatten. In allen Bedingungen sollte die Antwort auf die gestellte Frage wie in den Übungsaufgaben binnen 3 Sekunden mittels Anklicken einer von elf eine Ratingskala repräsentierenden Schaltflächen erfolgen. Durch Anklicken einer mit „Los!“ beschrifteten Schaltfläche wurde der entsprechende Durchgang der Testphase gestartet.

Die Stimuluspräsentation während der Testphase bestand aus der Darstellung einer Instanz des während der Lernphase gelernten Kausalsystems. Dabei wurden die verschiedenen Variablen des Systems mit ihren entsprechenden Ausprägungen einzeln und in zufälliger Reihenfolge in der Mitte des Bildschirms für kurze Zeit nacheinander präsentiert. Die Präsentationsdauer sollte gewährleisten, dass die dargebotenen Informationen bewusst aufgenommen werden konnten, sie sollte aber gleichzeitig ein längeres bewusstes Nachdenken über die Relevanz des präsentierten Stimulus für die zu beantwortende Frage verhindern. Die Versuchspersonen hatten während der Präsentation keine Möglichkeit der Einflussnahme. Sie konnten lediglich die präsentierte Sequenz von Variablenausprägungen betrachten. Der Ort für die Präsentation wurde so gewählt, da in keinem der mittels graphischer Abbildung instruierten Kausalsysteme an dieser Stelle eine Variable dargestellt wurde. Im Anschluss an die Präsentation der Variablen mit den der Instanziierung des Kausalsystems entsprechenden Ausprägungen erfolgte die Manipulation der kognitiven Verarbeitung mittels der oben beschriebenen bedingungsspezifischen Aufgaben, die automatisch begannen und nach Ablauf der vorgesehenen Zeit automatisch beendet wurden. Benötigten die Versuchspersonen für die im Anschluss abzugebenden Einschätzungen länger als das ihnen zur Verfügung stehende 3-sekündige Antwortintervall, so wurde ein Hinweisfenster eingeblendet, das die Versuchspersonen aufforderte, beim nächsten Durchgang schneller zu antworten. Nachdem eine Versuchsperson alle Durchgänge des Experimentes absolviert hatte wurde ein Bildschirm mit Informationen über den Zweck der Studie eingeblendet und ihr für die Teilnahme am Experiment gedankt.

\subsection{Experiment 1}

\subsubsection{Ziel von Experiment 1}

Mit dem im Folgenden vorgestellten Experiment sollte die Rolle von Typ1- bzw. Typ2-Verarbeitungsprozessen bei der Bearbeitung zweier verschiedener Aufgabenarten mit Bezug zu einem zuvor gelernten Kausalsystem untersucht werden. Dabei sollte zum einen 
geprüft werden, ob Entscheidungen und Urteile auf der Grundlage explizit vorhandenen Kausalwissens notwendigerweise Typ 2-Prozesse in Form bewusster Deliberation erfordern, oder ob sie auch spontan mittels Typ 1-Prozessen durchgeführt werden können. Zum anderen sollte untersucht werden, ob die Art der zu bearbeitenden kausalen Aufgabe mit den beiden Arten von Verarbeitungsprozessen in einem Zusammenhang steht. Bei den beiden verwendeten Aufgabenarten handelte es sich einerseits um Kategorisierungsurteile und andererseits um Interventionsentscheidungen. Ausgehend von den in Abschnitt 3.4 aufgestellten Hypothesen kann dabei angenommen werden, dass Kategorisierungsurteile auch ohne bewusste Deliberation möglich sein können, während Urteile über die Güte möglicher Interventionen mentale Simulationen von Interventionsfolgen erfordern und daher von einer Phase bewussten Nachdenkens profitieren sollten. Für die Kategorisierungsurteile wurde daher vorhergesagt, dass sich keine Vorteile von nach einer Phase bewussten Nachdenkens abgegebenen Urteilen gegenüber unmittelbar nach der Stimuluspräsentation abgegebenen Urteilen zeigen. Im Gegensatz dazu wurde für Interventionsurteile vorhergesagt, dass sie im Anschluss an eine Phase bewussten Denkens besser ausfallen als unmittelbar nach der Stimuluspräsentation.

Zur Untersuchung dieser Fragen wurde ein Kausalsystem konstruiert, dessen Struktur sowohl für Kategorisierungsurteile als auch für Interventionsentscheidungen genutzt werden kann. Um eventuelle Vorteile bewusster Deliberation untersuchen zu können, war das System zudem relativ komplex. Es bestand aus 8 Variablen, die durch 8 Kausalbeziehungen miteinander verbunden waren (vgl. Abbildung 5). Entscheidungen und Urteile auf Grundlage des kausalen Systems wurden mit drei unterschiedlichen experimentellen Bedingungen untersucht. In der Bedingung spontanen Entscheidens wurde unmittelbar nach der Präsentation eines auf dem System basierenden Falles ein Urteil bezüglich dessen Kategorienzugehörigkeit bzw. eine Entscheidung bezüglich der besten Intervention verlangt. In der Bedingung unbewussten Denkens hatten die Versuchspersonen nach der Präsentation des Falles hingegen eine 2-back-Aufgabe zu absolvieren bevor sie ihr Urteil abgeben sollten. In der Bedingung bewussten Denkens wurden die Versuchspersonen aufgefordert, vor der Abgabe ihres Urteils gründlich über ihre Entscheidung bezüglich des präsentierten Falles nachzudenken.

\subsubsection{Stichprobe und experimentelles Design}

Insgesamt nahmen 173 Versuchspersonen an Experiment 1 teil. Sie wurden zufällig auf die verschiedenen Bedingungen aufgeteilt. Das Experiment kann als 2 (Aufgabe: 
Kategorisierung vs. Intervention) X 3 (Verarbeitungsbedingung: Spontan vs. Unbewusst vs. Bewusst) X 8 (präsentierte Fälle) Design charakterisiert werden, wobei die Variation der Aufgaben sowie der Verarbeitungsbedingungen zwischen den Versuchspersonen, die Variation der Fälle hingegen innerhalb der Versuchspersonen erfolgte.

\subsubsection{Ablauf und verwendetes Stimulusmaterial}

Da der generelle Ablauf der im Rahmen dieser Arbeit vorgestellten Experimente bereits in Abschnitt 4.1 ausführlich dargestellt wurde, soll an dieser Stelle nur auf die Besonderheiten von Experiment 1 eingegangen werden. Im Anschluss an die Übungsphase wurde den Versuchspersonen die Coverstory für das verwendete Kausalsystem präsentiert. In der Coverstory ging es um Techniken und Fähigkeiten von Schamanen und wie diese miteinander in Beziehung stehen. Unabhängig von der Art der zu bearbeitenden Aufgabe (Kategorisierung oder Intervention) wurde für alle Versuchspersonen ein hinsichtlich Variablen- und Beziehungsstruktur identisches Kausalsystem verwendet (s. Abbildung 5). Dies bedeutet auch, dass allen Versuchspersonen unabhängig von der von ihnen zu bearbeitenden Aufgabenart dieselben Variablen mit denselben Variablenausprägungen präsentiert wurden.

Aufgrund der unterschiedlichen Aufgaben gab es jedoch einige kleinere Abweichungen in den Instruktionen für die beiden Aufgabenarten. So hatten die Versuchspersonen in den Bedingungen mit Kategorisierungsaufgaben die präsentierten Fälle hinsichtlich der Unterscheidung echter vs. falscher Schamane zu klassifizierten. Deshalb wurden ihnen die beiden Ausprägungen der verschiedenen Variablen des Systems als Techniken und Fähigkeiten vorgestellt, die für echte bzw. falsche Schamanen charakteristisch sind. Die Versuchspersonen in den Bedingungen mit Interventionsaufgaben sollten entscheiden, welche von zwei zur Auswahl stehender Interventionen geeigneter ist, möglichst viele Variablen zu beeinflussen. Daher wurden die Ausprägungen der verschiedenen Variablen des Systems in diesen Bedingungen als erfolgreiche bzw. wenig erfolgreiche Techniken und Fähigkeiten von Schamanenschülern vorgestellt. Die möglichen Interventionen waren zwei Kurse, von denen einer von einem Schamanenschüler besucht werden konnte, um seine Fähigkeiten zu verbessern. Darüber hinaus wurde die den Versuchspersonen dargebotene graphische Darstellung des Kausalsystems balanciert, um einen Einfluss der Position der Variablen in den Abbildungen des Systems auszuschließen. 
Ein weiterer auf den beiden Aufgabenarten basierender Unterschied in den Instruktionen besteht in der Beschreibung der Basisraten der Variablen des Kausalsystems. Den Versuchspersonen in den Bedingungen mit Kategorisierungsaufgabe wurde mitgeteilt, dass die Wahrscheinlichkeit des Auftretens einer für einen echten Schamanen charakteristischen Ausprägung einer Technik oder Fertigkeit bei ca. 80\% liegt, wenn es sich tatsächlich um einen echten Schamanen handelt. Analog dazu liegt die Wahrscheinlichkeit des Auftretens einer für einen falschen Schamanen charakteristischen Ausprägung einer Technik bzw. Fertigkeit ebenfalls bei ca. $80 \%$, wenn es sich tatsächlich um einen falschen Schamanen handelt ( $\mathrm{P}$ (charakteristische Ausprägung echter Schamane | echter Schamane $)=P($ charakteristische Ausprägung falscher Schamane $\mid$ falscher Schamane $)=0,8)$. Die Basisraten wurden so gewählt, um plausibel zu machen, dass auch ein echter Schamane hin und wieder Techniken und Fertigkeiten in der für falsche Schamanen charakteristischen Ausprägung beherrscht und umgekehrt. In den Bedingungen mit Interventionsaufgabe wurde den Versuchspersonen hingegen mitgeteilt, dass die Wahrscheinlichkeit, dass ein Schamanenschüler eine Technik bzw. Fertigkeit in der wenig erfolgreichen Ausprägung beherrscht bei ca. $80 \%$ liegt. Diese Wahrscheinlichkeit wurde gewählt, um einerseits deutlich zu machen, dass bei Schamanenschülern in der Regel wenig erfolgreiche Techniken und Fertigkeiten vorherrschen. Andererseits sollte aber auch die Möglichkeit bestehen, dass ein Schamanenschüler bereits bestimmte erfolgreiche Techniken und Fertigkeiten beherrscht.

Um einen besseren Eindruck der den Versuchspersonen vermittelten Instruktionen bezüglich des Kausalsystems zu bekommen werden im Folgenden zunächst die Instruktionen für die drei Versuchsbedingungen mit Kategorisierungsaufgaben wiedergegeben. Im Anschluss werden dann die Instruktionen für die drei Versuchsbedingungen mit Interventionsaufgaben dargestellt.

\subsubsection{Instruktionen Kausalsystem Kategorisierungsaufgaben}

„, Schamanen sind „Mittler zwischen den Welten“. Ihre besondere Fähigkeit liegt darin, in Träumen und Visionen die Botschaften der spirituellen Welt wahrnehmen zu können. Um diese Botschaften einzufangen, machen echte Schamanen von verschiedenen Techniken und Fertigkeiten Gebrauch, die es ihnen ermöglichen, mit den Yuwipis, ihren Göttern, Kontakt aufzunehmen. Leider ist die Anzahl von Schwindlern, die sich auch als Schamanen ausgeben, um damit Geld zu verdienen, in letzter Zeit extrem gestiegen. Diese falschen Schamanen machen von ähnlichen Techniken Gebrauch, wie die echten Schamanen. Dennoch kann auch ein Laie zwischen echten und falschen Schamanen durch genaue Beobachtung der eingesetzten Techniken unterscheiden. Zu den wichtigen Techniken zählen Atemtechnik (Rekapulation vs. Hyperventilation), Gesang (rhythmisch vs. unrhythmisch), Tanz (Trancetanz vs. Sonnentanz), Beherrschung der Göttersprache (gut vs. schlecht), Wissen 
über die Regeln der Yuwipis (tiefgreifend vs. oberflächlich), Gebrauch psychedelischer Pflanzen (angemessen vs. ausartend), Rauchtechnik der heiligen Pfeife (inhalierend vs. paffend) und Dauer von Hunger-Meditationen (lang vs. kurz).

Echte Schamanen zeichnen sich durch folgende Merkmale aus: Ca. $80 \%$ nutzen Rekapulation als Atemtechnik; Ca. 80\% haben einen rhythmischen Gesang; Ca. 80\% verwenden den Trancetanz; Ca. $80 \%$ beherrschen die Göttersprache gut; Ca. 80\% haben tiefgreifendes Wissen über die Regeln der Yuwipis; Ca. 80\% haben einem angemessenen Gebrauch psychedelischer Pflanzen; Ca. 80\% rauchen inhalierend die heilige Pfeife; Ca. 80\% unternehmen lange Hunger-Meditationen; Schwindler beherrschen nur vereinzelt diese Techniken;

Falsche Schamanen setzen folgende Techniken ein: Ca. $80 \%$ nutzen Hyperventilation als Atemtechnik; Ca. $80 \%$ haben einen unrhythmischen Gesang; Ca. $80 \%$ verwenden den Sonnentanz; Ca. $80 \%$ beherrschen die Göttersprache schlecht; Ca. 80\% haben oberflächliches Wissen über die Regeln der Yuwipis; Ca. 80\% haben einen ausartenden Gebrauch psychedelischer Pflanzen; Ca. 80\% rauchen paffend die heilige Pfeife; Ca. $80 \%$ unternehmen kurze Hunger-Meditationen; Echte Schamanen verwenden in seltenen Fällen diese Techniken. “

Zusätzlich wurde den Versuchspersonen das Kausalsystem auch graphisch präsentiert. Diese Art der Präsentation diente zum einen der Veranschaulichung der Struktur des verwendeten Kausalsystems. Zum anderen wurden die kausalen Relationen zwischen den Variablen in den Abbildungen explizit benannt (z.B. „Rekapulation führt zu rhytmischem Gesang"). Insgesamt bestand der Zweck der Präsentation der graphischen Systemdarstellungen in einer Vereinfachung des Lernens für die Versuchspersonen. In drei aufeinanderfolgenden Graphiken wurde erstens dargestellt, wie sich die Techniken von echten Schamanen gegenseitig bedingen (s. Abbildung 3). Zweitens wurde dargestellt, wie sich die Techniken von falschen Schamanen gegenseitig bedingen (s. Abbildung 4). Drittens wurde ein Überblick über die Variablen des Kausalsystems, ihre möglichen Ausprägungen, sowie die Kausalrelationen zwischen ihnen präsentiert (s. Abbildung 5). Aus den Abbildung 3 und 4 ist auch ersichtlich, wie das verwendete Kausalsystem hinsichtlich der Position der verschiedenen Variablen zwischen den Versuchspersonen balanciert wurde. 
Tanz:

Trancetanz

Der rhythmische

Gesang führt zu Trance

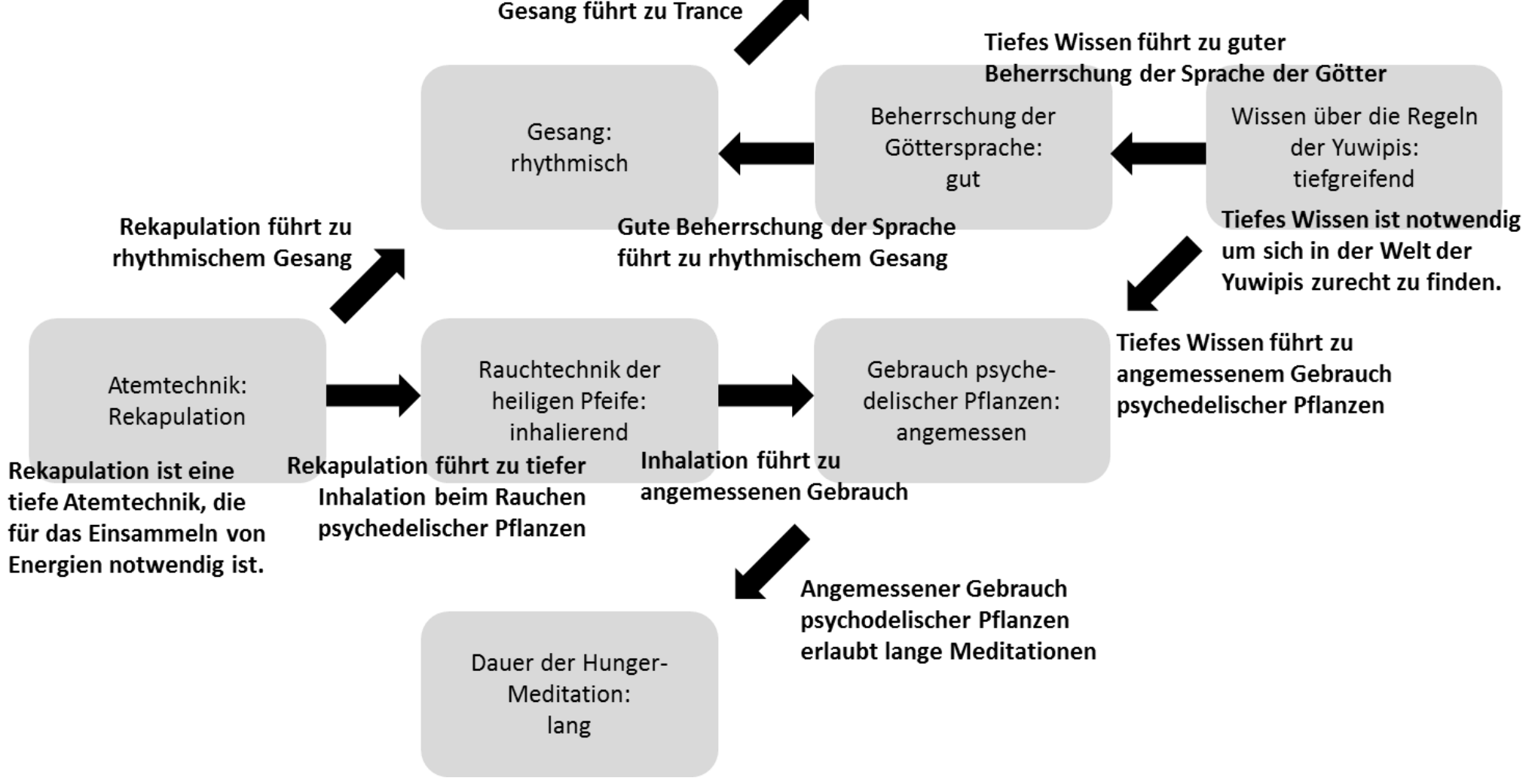

Abbildung 3: Darstellung des Kausalsystems, wie es in den Verarbeitungsbedingungen mit Kategorisierungsurteil in Experiment 1 verwendet wurde. Die Darstellung zeigt die für einen echten Schamanen charakteristischen Variablenausprägungen, sowie eine Erklärung der bestehenden Kausalrelationen in Balancierungsbedingung 1. Die zusammen mit dieser Darstellung präsentierte Überschrift lautete in den Kategorisierungsbedingungen: „Die von den Schamanen eingesetzten Techniken sind kausal miteinander verbunden. Diese Abbildung erklärt wie sich die Techniken der ECHTEN Schamanen gegenseitig bedingen“. In den Interventionsbedingungen lautete die Überschrift: „Die von den Schamanen eingesetzten Techniken sind kausal miteinander verbunden. Diese Abbildung erklärt wie sich die erfolgreichen Techniken gegenseitig bedingen". 


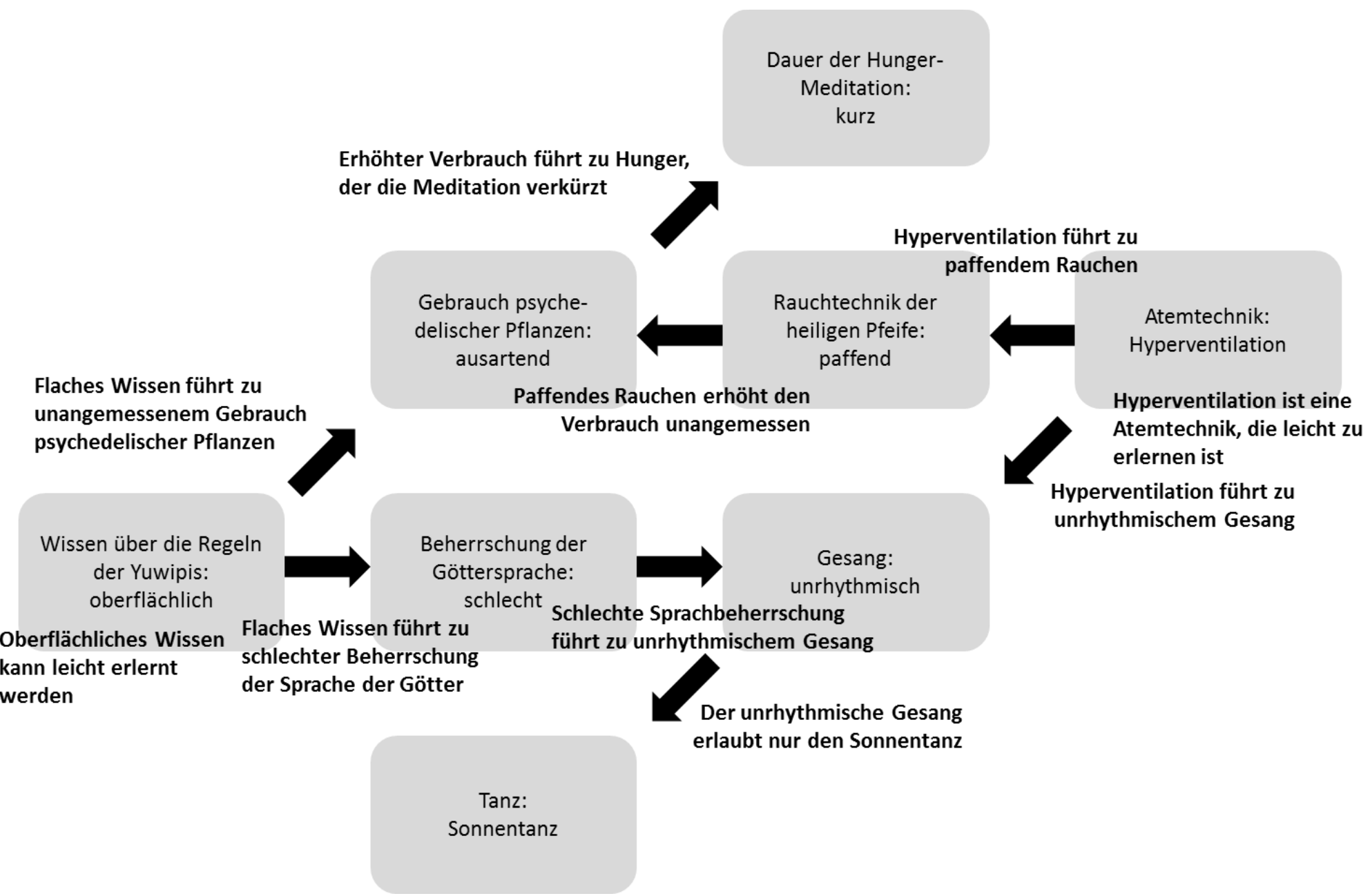

Abbildung 4: Darstellung des Kausalsystems, wie es in den Verarbeitungsbedingungen mit Kategorisierungsurteil in Experiment 1 verwendet wurde. Die Darstellung zeigt die für einen falschen Schamanen charakteristischen Variablenausprägungen, sowie eine Erklärung der bestehenden Kausalrelationen in Balancierungsbedingung 2. Die zusammen mit dieser Darstellung präsentierte Überschrift lautete in den Kategorisierungsbedingungen: „Die von den Schamanen eingesetzten Techniken sind kausal miteinander verbunden. Diese Abbildung erklärt wie sich die Techniken der FALSCHEN Schamanen gegenseitig bedingen“. In den Interventionsbedingungen lautete die Überschrift: „Die von den Schamanen eingesetzten Techniken sind kausal miteinander verbunden. Diese Abbildung erklärt wie sich die wenig erfolgreichen Techniken gegenseitig bedingen“" 


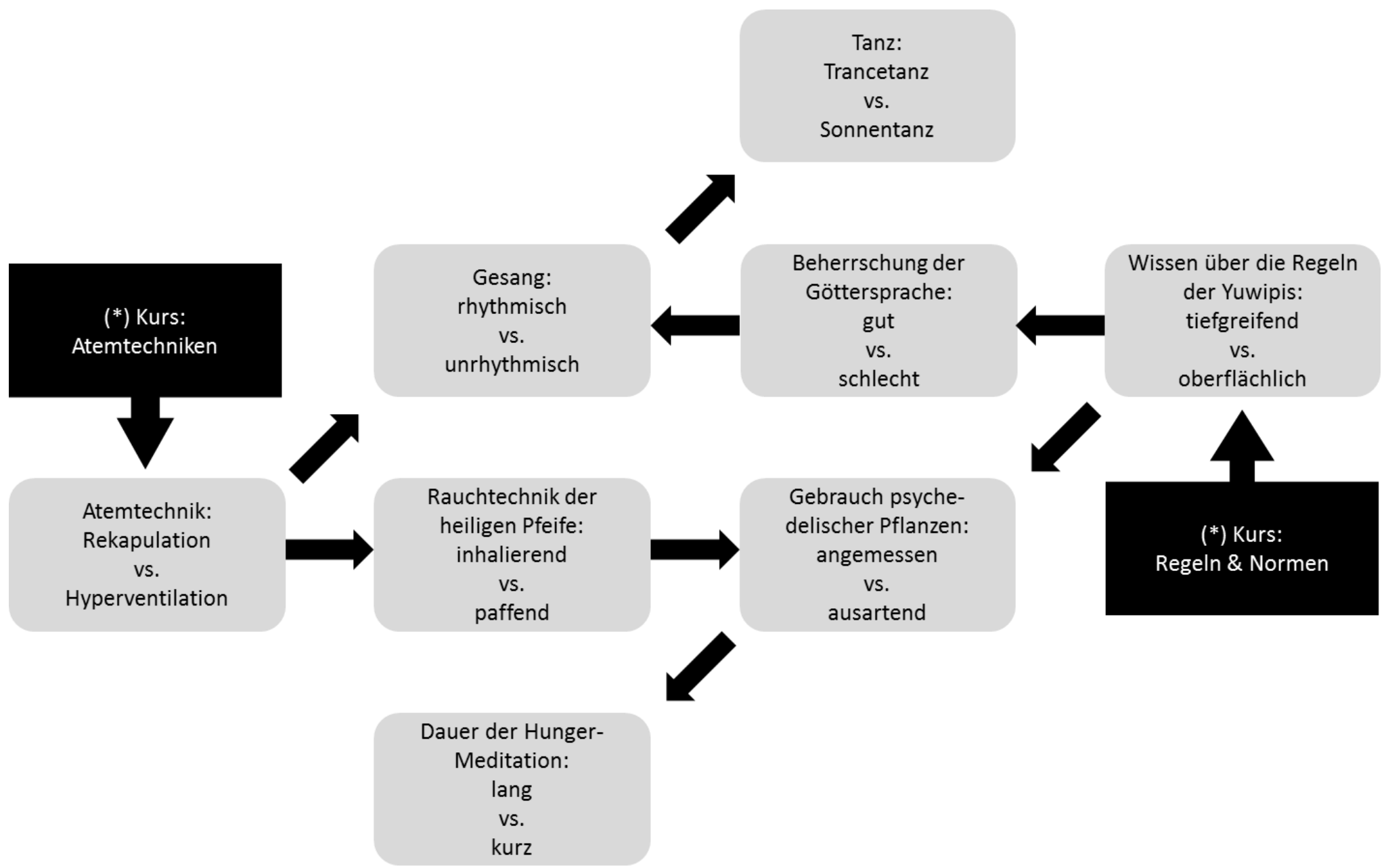

Abbildung 5: Darstellung des in Experiment 1 verwendeten Kausalsystems mit möglichen Variablenausprägungen und Kausalrelationen. Die mit $(*)$ gekennzeichneten schwarzen Felder stellen die beiden möglichen Interventionen dar und wurden ausschließlich den Versuchspersonen in den Verarbeitungsbedingungen mit Interventionsaufgabe präsentiert. Die zusammen mit dieser Darstellung präsentierte Überschrift lautete in den Kategorisierungsbedingungen: „Diese Abbildung stellt einen Überblick über das Kausalsystem dar.“ In den Interventionsbedingungen lautete die Überschrift: „Diese Abbildung zeigt, worauf sich die beiden Kurse der Schamanenmeister auswirken“. 


\subsubsection{Instruktionen Kausalsystem Interventionsaufgaben}

Den Versuchspersonen, die im Anschluss an die verschiedenen Verarbeitungsbedingungen die bessere von zwei möglichen Interventionen bestimmen sollten wurden folgende Instruktionen präsentiert:

„Schamanen sind „Mittler zwischen den Welten“. Ihre besondere Fähigkeit liegt darin, in Träumen und Visionen die Botschaften der spirituellen Welt wahrnehmen zu können. Um diese Botschaften einzufangen, machen echte Schamanen verschiedener Techniken und Fertigkeiten Gebrauch, die es ihnen ermöglichen, mit den Yuwipis, ihren Göttern, Kontakt aufzunehmen: Atemtechnik, Gesang, Tanz, Beherrschung der Göttersprache, Wissen über die Regeln der Yuwipis, Gebrauch psychedelischer Pflanzen, Rauchtechnik der heiligen Pfeife und Dauer der Hunger-Meditation. Einige Formen dieser Techniken und Fertigkeiten haben sich als erfolgreicher und andere als weniger erfolgreich erwiesen.

Als erfolgreichere Techniken und Fertigkeiten gelten: Rekapulation als Atemtechnik; rhythmischer Gesang; Trancetanz; eine gute Beherrschung der Göttersprache; tiefgreifendes Wissen über die Regeln der Yuwipis; ein angemessener Gebrauch psychedelischer Pflanzen; eine inhalierende Rauchtechnik der heiligen Pfeife; lange Hunger-Meditationen.

$\mathrm{Zu}$ den weniger erfolgreichen Techniken und Fertigkeiten gehören: Hyperventilation als Atemtechnik; unrhythmischer Gesang; Sonnentanz; eine schlechte Beherrschung der Göttersprache; oberflächliches Wissen über die Regeln der Yuwipis; ein ausartender Gebrauch psychedelischer Pflanzen; eine paffende Rauchtechnik der heiligen Pfeife; kurze Hunger-Meditationen.

Die Wahrscheinlichkeit dafür, dass ein Schamanenschüler eine der weniger erfolgreichen Techniken oder Fertigkeiten anwendet liegt bei ca. 80\%, d.h. man findet bei ca. $80 \%$ der Schamanenschülern Hyperventilation als Atemtechnik, bei ca. $80 \%$ unrhythmischen Gesang, bei ca. $80 \%$ den Sonnentanz, bei ca. 80\% eine schlechte Beherrschung der Göttersprache, bei ca. $80 \%$ oberflächliches Wissen über die Regeln der Yuwipis, bei ca. $80 \%$ einen ausartenden Gebrauch psychedelischer Pflanzen, bei ca. $80 \%$ eine paffende Rauchtechnik der heiligen Pfeife und bei ca. $80 \%$ eine kurze Hunger-Mediation.

Schamanenschüler werden von Schamanenmeistern unterrichtet. Diese führen zwei „Kurse“ durch. Da die Kurse jedoch zur selben Zeit stattfinden, kann jeder Schamanenschüler nur an einem der beiden Kurse teilnehmen. Folgende Kurse werden angeboten:

- Regeln und Normen: ein Kurs, indem die Regeln der Yuwipis erklärt und eingeübt werden, sodass das ein tiefes Wissen über diese Regeln entsteht.

- Atemtechniken: ein Kurs, in dem den Schamanen die Rekapulation als Atemtechnik beigebracht und intensiv geübt wird. “ 
Auch in den Bedingungen mit Interventionsaufgaben wurde das Kausalsystem mittels dreier Graphiken verdeutlich. Die ersten beiden Darstellungen des Kausalsystems waren dabei (mit Ausnahmen einer kleinen Modifikation der Überschrift gemäß der Unterschiede in den Instruktionen) mit den ersten beiden Abbildungen in den Bedingungen mit Kategorisierungsaufgaben identisch (vgl. Abbildung 3 ). In der dritten Graphik war zusätzlich zur zusammenfassenden Darstellung des Kausalsystems markiert, auf welche Variablen sich die beiden Kurse der Schamanenmeister auswirken (die mit (*) markierten Felder in Abbildung 5).

\subsubsection{Multiple-Choice-Wissenstest}

Wie bereits im Abschnitt über die Gemeinsamkeiten des Ablaufs der vorgestellten Experimente beschrieben folgte auf die Instruktion des Kausalsystems ein Multiple-ChoiceWissenstest. Mit diesem sollte sichergestellt werden, dass die Versuchspersonen das instruierte Kausalsystem richtig verstanden hatten. Die Fragen und Antwortmöglichkeiten der Multiple-Choice-Tests für die beiden Aufgabenarten in Experiment 1 befindet sich in Anhang A. Die jeweils richtigen Antworten sind dabei markiert. Insgesamt bestand der Wissenstest aus 10 Fragen mit jeweils 4 Antwortmöglichkeiten, von denen jeweils eine Antwort korrekt war. Die Versuchspersonen mussten alle 10 Fragen korrekt beantworten, um zur nächsten Phase des Experimentes zu gelangen.

\subsubsection{Testphase}

Sobald eine Versuchsperson alle Multiple-Choice-Fragen korrekt beantwortet hatte gelangte sie in die Testphase, die bei Experiment 1 aus acht Durchgängen bestand. In jedem Durchgang wurde zunächst die zu beantwortende Frage für ca. 3 Sekunden präsentiert. Dabei wurde die Reihenfolge der Nennung der beiden Kategorien sowie die Anker in den zugehörigen Ratingskalen ausbalanciert, um mögliche Richtungseffekte bei der Abgabe der Einschätzungen zu kontrollieren. In den Bedingungen mit Kategorisierungsaufgaben lautete diese für 39 der 77 Versuchspersonen: „Handelt es sich um einen echten oder einen falschen Schamanen?“. Für diese Versuchspersonen war der linke Endpunkt der elfstufigen Antwortskala, auf der sie im Anschluss an die Präsentation des zu beurteilenden Falles und die ggf. darauf folgende 2-back- bzw. Nachdenk-Aufgabe ihre Einschätzung abgeben sollten mit „sicher ein echter Schamane“ beschriftet. Der rechte Endpunkt der Skala war entsprechend mit „sicher ein falscher Schamane“ beschriftet. Die elf Schaltflächen, mittels derer die Versuchspersonen ihre Einschätzungen abgeben konnten waren in allen 
Bedingungen von Experiment 1 von links nach rechts mit Zahlen von 0 bis 100 (in 10er Schritten) beschriftet. Den verbleibenden 38 Versuchspersonen wurde die Frage in leicht veränderter Form („Handelt es sich um einen falschen oder echten Schamanen?“) präsentiert. Für diese Versuchspersonen war der linke Endpunkt der Antwortskala mit ,sicher ein falscher Schamane“ und der rechte Endpunkt mit „sicher ein echter Schamane“ beschriftet.

Der Hälfte $(n=38)$ der Versuchspersonen in den Bedingungen mit Interventionsaufgaben wurde in der Testphase die folgende Frage präsentiert: „Würdest Du den Schamanen in den Regeln und Normen-Kurs oder in den Atemtechniken-Kurs schicken?“. Der linke Endpunkt der Antwortskala war mit „sicher Regeln und Normen“, der rechte Endpunkt mit ,sicher Atemtechniken“ beschriftet. Der zweiten Hälfte der Versuchspersonen $(n=38)$ wurde die Frage in balancierter Form (,Würdest Du den Schamanen in den Atemtechniken-Kurs oder in den Regeln und Normen-Kurs schicken?“) präsentiert. Der linke Endpunkt der Antwortskala für diese Gruppe von Versuchspersonen war mit „sicher Atemtechniken“, der Rechte mit „,sicher Regeln und Normen“ beschriftet.

Die acht von den Versuchspersonen zu beurteilenden Fälle waren ebenso wie ihre Präsentation in allen Bedingungen von Experiment 1 identisch: Zunächst wurde die Präsentationsreihenfolge der Fälle mittels eines Zufallsalgorithmus für jede Versuchsperson individuell festgelegt. Für jeden einzelnen Versuchsdurchgang bzw. Fall wurde die Reihenfolge der zu präsentierenden Ausprägungen der Variablen des Kausalsystems individuell mittels eines weiteren Zufallsalgorithmus bestimmt. Vor Beginn eines jeden Versuchsdurchganges wurden auf dem Bildschirm Instruktionen bezüglich der folgenden Aufgabe präsentiert (vgl. Abschnitt 4.1). Die Dauer der Präsentation der Ausprägungen der verschiedenen Variablen des Kausalsystems betrug in Experiment 1 ca. 1,5 Sekunden. Diese Dauer wurde gewählt, weil sie sich in Vortests als ausreichend zum Erfassen der Information erwies und dabei nicht viel Zeit zum Nachdenken erlaubte. Nach Ablauf dieser Zeit wurde automatisch die nächste Ausprägung eingeblendet. Die Präsentation der Ausprägungen erfolgte dabei in der Mitte des Bildschirms und entsprach optisch der den Versuchspersonen aus den graphischen Darstellungen des Kausalsystems während der Lernphase bekannten Form. Nach der Präsentation aller Variablenausprägungen aller Fälle wurde in der Bedingung unbewussten Denkens und der Bedingung bewussten Denkens die entsprechende Aufgabe automatisch gestartet und nach 2 Minuten automatisch beendet. Danach erfolgte die Abfrage der Einschätzung des präsentierten Falles hinsichtlich seiner Kategorienzugehörigkeit bzw. der geeigneteren Intervention. Wurde die Einschätzung nicht innerhalb von drei Sekunden 
abgegeben, so erfolgte im Unterschied zu den anderen im Rahmen dieser Arbeit vorgestellten Experimenten kein unmittelbarer Hinweis, beim nächsten Mal schneller zu antworten. Stattdessen wurde im anschließenden Versuchsdurchgang unmittelbar vor der Abfrage der Einschätzung ein Hinweisfenster mit dem Text „Bitte denke daran, dass Du für die nun folgende Einschätzung nur max. 3 Sekunden Zeit hast!“. Daraufhin wurde das Experiment automatisch fortgesetzt.
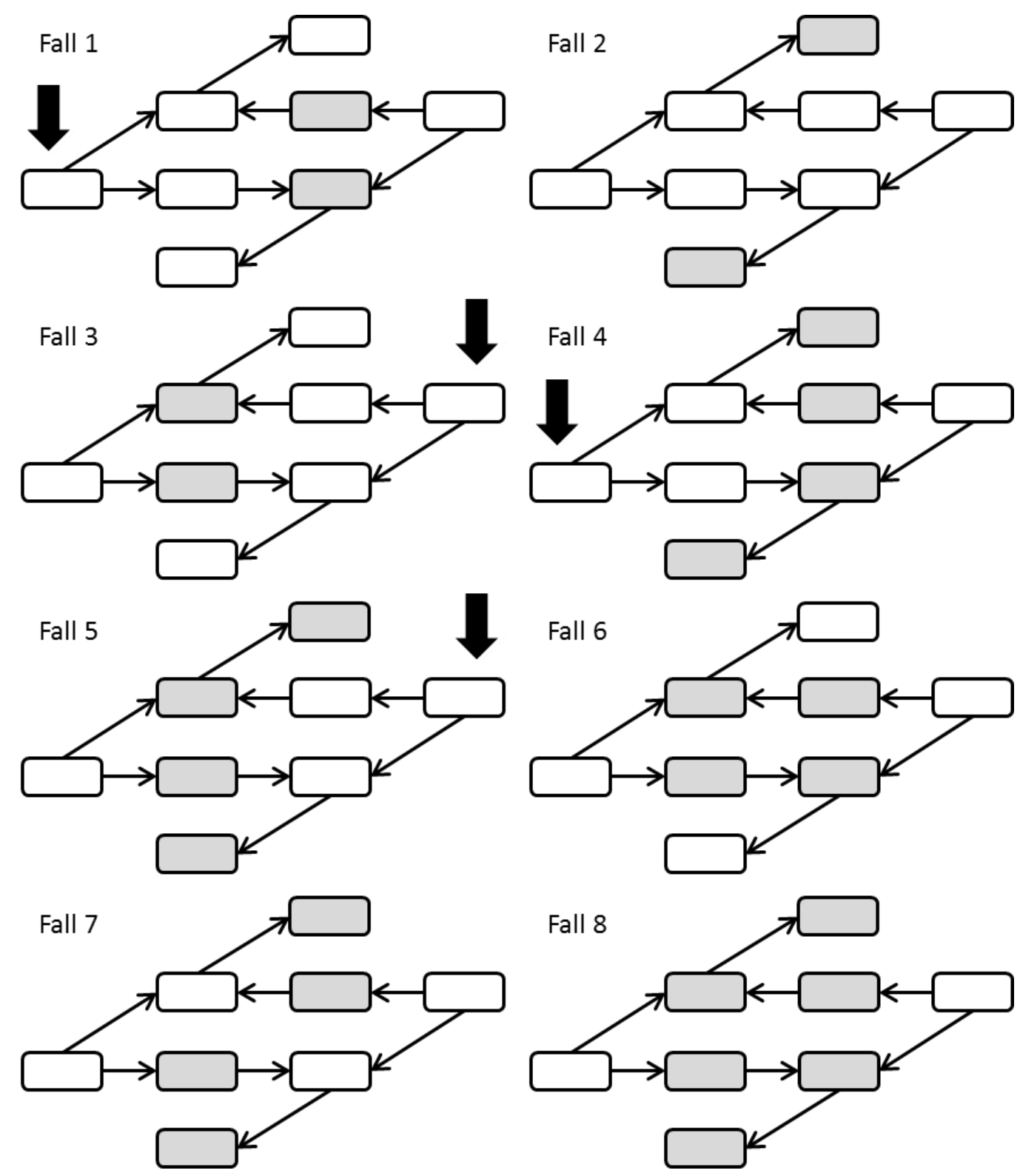

Abbildung 6: Schematische Darstellung der In Experiment 1 präsentierten Fälle (Abbildung gemäß des in Balancierungsbedingung 1 verwendeten Kausalsystems). Die Felder repräsentieren die Variablen des Kausalsystems, die Pfeile die kausalen Verbindungen zwischen den Variablen, wobei die Pfeilspitze auf den Effekt in der jeweiligen Relation weist. Grau gefärbte Felder repräsentieren für die Kategorisierungsbedingungen diejenigen Techniken und Fertigkeiten, die charakteristisch für einen echten Schamanen sind und für die Interventionsbedingungen die erfolgreichen Techniken und Fertigkeiten. In den Fällen 1, 3, 4 und 5 ist die für den jeweiligen Fall beste Intervention als zusätzliches schwarz gefärbtes Feld in Pfeilform dargestellt. Diese Felder haben für die Kategorisierungsbedingungen keine Relevanz. 
Die in Experiment 1 präsentierten Fälle werden in Abbildung 6 graphisch dargestellt. Da sowohl für die Kategorisierungs- als auch für die Interventionsaufgabe dieselben Fälle verwendete werden sollten, wurde sichergestellt, dass die Variablen, die durch die beiden in der Interventionsaufgabe möglichen Interventionen unmittelbar beeinflusst werden konnten, jeweils die Ausprägung der wenig erfolgreichen Technik bzw. die für einen falschen Schamanen charakteristische Ausprägung aufwiesen.

Für die im Folgenden vorgestellten Vorhersagen für auf Grundlage des instruierten Kausalwissens gefällte Urteile gilt allgemein, dass die in den Instruktionen erwähnten Parameter des Kausalsystems aufgrund des symmetrischen Aufbaus sowie der Auswahl der in den verschiedenen Fällen vorhandenen Variablenausprägungen außer Acht gelassen werden können. Dies gilt sowohl für Kategorisierungs- als auch für Interventionsurteile. Werden Kategorisierungen auf der Grundlage des kausalen Status der Variablen im instruierten Kausalsystems vorgenommen, so sollte Fall 2 am ehesten der Kategorie „falscher Schamane“ zugeordnet werden, da er nur zwei für einen echten Schamanen charakteristische Variablenausprägungen aufweist und diese zudem die mit dem niedrigsten kausalen Status versehenen Positionen im verwendeten Kausalsystem einnehmen. Die Fälle 1 und 3 sollten ebenfalls als falsche Schamanen klassifiziert werden, da sie ebenfalls nur zwei für einen echten Schamanen charakteristischen Variablenausprägungen aufweisen, die allerdings im Vergleich zu Fall 2 einen höheren kausalen Status besitzen. Daher sollten Klassifikation der Fälle 1 und 3 als falsche Schamanen tendenziell weniger stark ausgeprägt sein als die Einschätzung von Fall 2. Die Fälle 4, 5, 6 und 7 weisen jeweils vier für einen echten Schamanen charakteristische Variablenausprägungen auf. Dabei ist der kausale Status der in den Fällen 4, 5 und 7 vorhandenen für einen echten Schamanen charakteristischen Variablenausprägung etwas geringer als in Fall 6, der daher von diesen vier Fällen am ehesten als echter Schamane klassifiziert werden sollte. Fall 8 weist insgesamt sechs für einen echten Schamanen charakteristische Variablenausprägungen auf und sollte deshalb von allen präsentierten Fällen am stärksten als echter Schamane klassifiziert werden. Auf Grundlage des kausalen Status ergibt sich somit insgesamt folgende Vorhersage bezüglich der Kategorisierung der Fälle als echte Schamanen: Fall $8>$ Fall $6>$ Fälle 4,5 \& $7>$ Fälle 1\&3> Fall 2. Für Kategorisierungen auf Grundlage der kausalen Kohärenz der präsentierten Fälle ergibt sich ein ähnliches Bild. Für die Vorhersage wird die Anzahl der Kausalrelationen gezählt, bei denen beide beteiligte Variablen die gleiche Ausprägung aufweisen und damit kohärent mit dem Kausalsystem sind. Haben beide Variablen die für einen echten Schamanen charakteristische Ausprägung, so erhöht diese Kausalrelation die Wahrscheinlichkeit einer 
Kategorisierung des Falles in dem sie auftritt als echter Schamane. Haben beide Variablen hingegen die für einen falschen Schamanen charakteristische Ausprägung, so erhöht sich die Wahrscheinlichkeit einer Kategorisierung als falscher Schamane. Die Vorhersagen für die einzelnen Fälle ergeben sich aus den in ihnen vorkommenden kohärenten Relationen für einen echten bzw. einen falschen Schamanen. Die Vorhersagen entsprechen weitestgehend denen auf Grundlage des kausalen Status. Lediglich der Fall-Cluster 4, 5 \& 7 wird in zwei Elemente gesplittet und davon ausgegangen, dass die Fälle 4 und 5 eher als echter Schamane kategorisiert werden als Fall 7. Insgesamt ergibt sich somit folgende Vorhersage bezüglich der Fälle als echte Schamanen (in Klammern das Verhältnis der mit einem echten bzw. einem unechten Schamanen kohärenten Relationen): Fall 8 (4:0) > Fall 6 (2:0) > Fälle 4\&5 (1:2) > Fall $7(0: 2)>$ Fälle $1 \& 3(0: 3)>$ Fall $2(0: 6)$.

In den Bedingungen mit Interventionsaufgaben kommt es darauf an, diejenige Intervention zu wählen, die auf Basis des instruierten Kausalsystems das Potenzial hat, die meisten wenig erfolgreichen Techniken und Fertigkeiten eines Schamanenschülers in erfolgreiche Techniken und Fertigkeiten umzuwandeln. Die Fälle 2, 6, 7 und 8 sind diesbezüglich indifferent, da beide möglichen Interventionen jeweils gleich viele Techniken und Fertigkeiten beeinflussen können ( 4 bei Fall 2; 1 bei Fall 6 und Fall 8; 2 bei Fall 7). Hingegen sind die Fälle 1, 3, 4 und 5 geeignet, um zwischen den möglichen Interventionen zu differenzieren. In Fall 1 ist Intervention A (der Kurs „Atemtechniken) zu bevorzugen, die 4 Variablen potenziell beeinflussen kann, während Intervention B (der Kurs: „Regeln \& Normen") hier lediglich eine Variable beeinflussen kann. Für Fall 3 ergibt sich ein Vorteil von Intervention $\mathrm{B}$ mit 4 potenziell beeinflussbaren Variablen gegenüber einer beeinflussbaren Variablen bei Intervention A. In Fall 4 ergibt sich ein Vorteil von 3:1 für Intervention A, bei Fall 5 ein 3:1-Vorteil für Intervention B. Insgesamt ergeben sich auf Grundlage des instruierten Kausalsystems und Hypothese 2 für die Interventionsaufgaben somit folgende Erwartungen im Hinblick auf die Wahl von Intervention A als besserer Alternative: Fall 1> Fall $4>$ Fälle 2, 6, $7 \& 8>$ Fall $5>$ Fall 3. Diese Vorhersage besteht auch dann unverändert fort, wenn man zusätzlich den kausalen Status der jeweiligen beeinflussbaren Variablen berücksichtigt. Die kausale Kohärenz übt auf die Abschätzung der Interventionsfolgen keinen Einfluss aus.

\subsubsection{Ergebnisse}

Die Daten von 153 der ursprünglichen 173 Versuchspersonen gingen in die Analyse von Experiment 1 ein. Diese Reduzierung kann wie folgt erklärt werden: Bei sechs 
Versuchspersonen wurden (aufgrund von Abstürzen des Computerprogramms) außer den demographischen keine weiteren Daten erfasst. 8 Versuchspersonen scheiterten am MultipleChoice Wissenstest und weitere 6 Versuchspersonen brachen das Experiment vorzeitig ab. Von den verbleibenden 153 Versuchspersonen waren 111 weiblichen und 42 männlichen Geschlechts. Diese Versuchspersonen waren Studenten der Universität Göttingen aus verschiedenen Fachrichtungen, wobei Studierende der Psychologie mit 63 Versuchspersonen $(41,2 \%)$ die mit Abstand größte Gruppe bildete. Die Analyse der Kategorisierungsbedingungen basiert auf den Daten von77 Versuchspersonen. Von diesen waren 30 der Bedingung spontanen Entscheidens, 23 der Bedingung unbewussten Nachdenkens und 24 der Bedingung bewussten Denkens zugeteilt. 76 Versuchspersonen lieferten verwertbare Daten in den Bedingungen mit Interventionsaufgaben. Hiervon waren 31 der Bedingung spontanen Entscheidens, 22 der Bedingung unbewussten und 23 der Bedingung bewussten Nachdenkens zugeteilt. Die Versuchspersonen benötigten im Durchschnitt etwa 30 Minuten für Experiment 1. Aufgrund der unterschiedlichen Anforderungen der verschiedenen Verarbeitungsbedingungen war die durchschnittliche Dauer mit ca. 16 Minuten in der Bedingung spontanen Entscheidens (Kategorisierung: ca. 18 Minuten; Intervention: ca. 16 Minuten) deutlich kürzer als in den Bedingungen unbewussten Denkens (Kategorisierung: ca. 39 Minuten; Intervention: ca.41 Minuten) bzw. bewussten Nachdenkens (Kategorisierung: ca. 37 Minuten; Intervention: ca. 39 Minuten).

\subsubsection{Multiple-Choice Wissenstest}

Insgesamt konnten 8 von 167 Versuchspersonen auch nach sechs Durchgängen nicht alle Fragen des Wissenstests korrekt beantworten, was einer Quote von 5\% entspricht. Durchschnittlich benötigten die Versuchspersonen 1,78 Durchgänge, um die Fragen bezüglich des für Experiment 1 relevanten Kausalsystems korrekt zu beantworten (s. Abbildung 7). Weder die Art der Aufgabe $(F(1,153)<1, p=0,36, M S E=1)$ noch die Verarbeitungsbedingung $(F(2,153)<1, \quad p=0,39, \quad M S E=1) \quad$ oder die Interaktion zwischen Aufgabenart und Verarbeitungsbedingung $(F(2,153)<1, p=0,69, M S E<1)$ hatten einen Einfluss auf die Anzahl der benötigten Durchgängen. 


\section{Durchschnittliche Anzahl der in Experiment 1 benötigten Lerntrials}

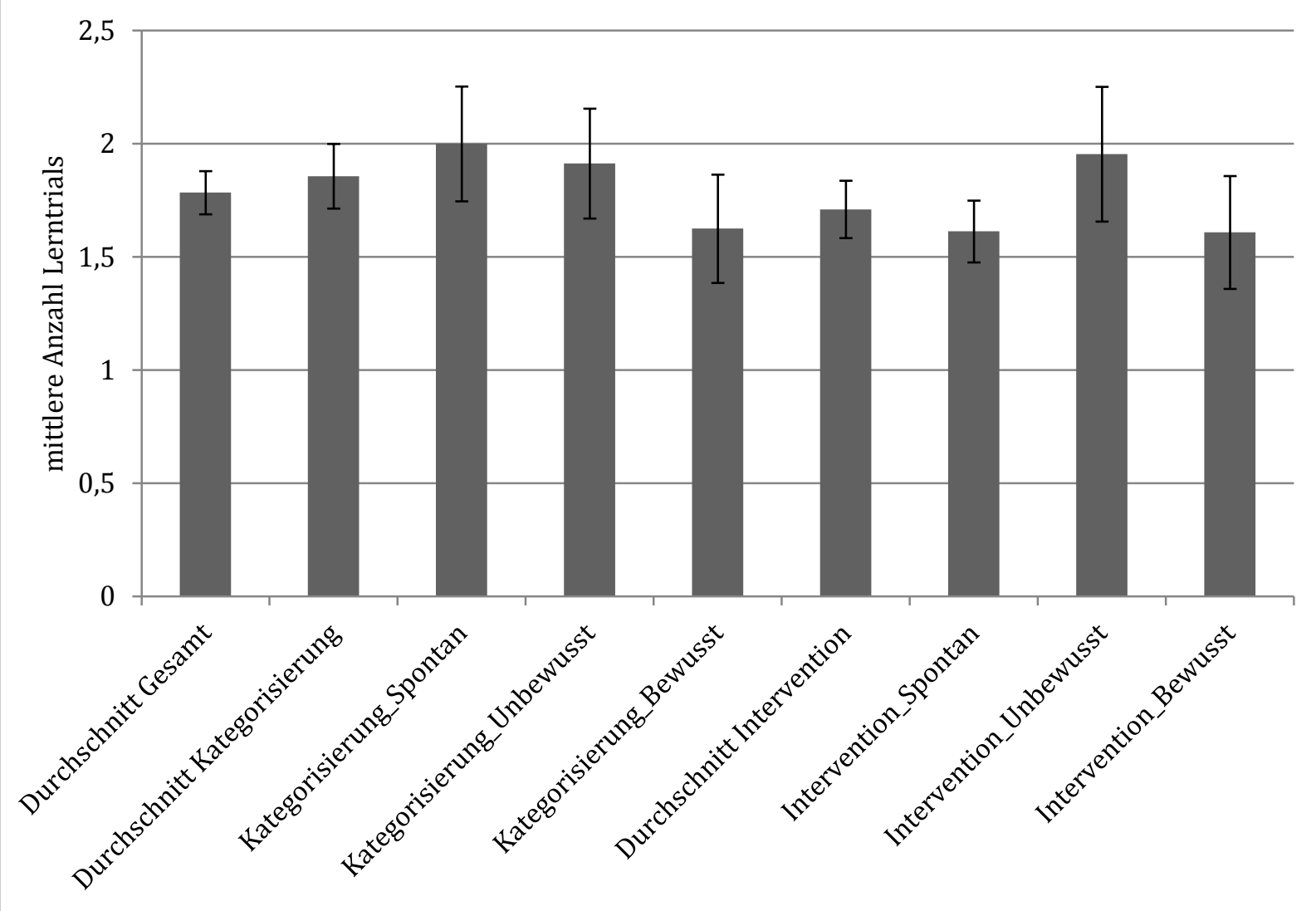

Abbildung 7: Durchschnittliche Anzahl der von den Versuchspersonen in Experiment 1 benötigten Durchgänge (und zugehörige Standardfehler) beim Multiple-Choice Wissenstests.

\subsubsection{Kategorisierungsurteile}

Für die nun vorzustellenden Analysen wurden zunächst auf individueller Ebene Ratings für Fälle mit gleichem erwarteten Kategorisierungsurteil gemäß der auf Grundlage des kausalen Status abgeleiteten Vorhersagen gemittelt. Dies führte dazu, dass für die Verarbeitungsbedingungen mit Kategorisierungsaufgaben zunächst fünf verschiedene Cluster von Fällen gebildet wurden (Fall 2; Fall 1\&3; Fall 4,5\&7; Fall 6; Fall 8 vgl. Abschnitt 2.4.2.3). Auf die Darstellung der Analyse der auf Grundlage der kausalen Kohärenz abgeleiteten Vorhersagen wird an dieser Stelle verzichtet, da sie aufgrund der geringen Unterschiede $\mathrm{zu}$ identischen Ergebnissen führte (s. Anhang B). Darüber hinaus gingen diejenigen Urteile in die Analysen ein, die die Versuchspersonen innerhalb von 5 Sekunden nach Erscheinen der Antwortskala abgegeben hatten. In dieses 5-Sekunden-Intervall geht auch die Dauer der Betrachtung eines etwaigen Hinweisfensters unmittelbar vor der Präsentation der Antwortskala mit ein. Ein solches wurde eingeblendet, wenn eine Versuchsperson im vorangegangenen Testdurchgang länger als 3 Sekunden für ihre Antwort 
benötigt hatte. Durch die Addition der Betrachtungszeit zur für die Abgabe des Urteils benötigten Zeit sollte sichergestellt werden, dass die Versuchspersonen die Einblendung des Fensters nicht für zusätzliches Nachdenken nutzen konnten.

Der Grund für die Analyse von Daten auf Grundlage eines 5-Sekunden-Intervalls liegt in einer Erhöhung der Anzahl der für die Analyse zur Verfügung stehenden Datenpunkte. Es wurde jedoch sichergestellt, dass sich durch die Hinzunahme der Urteile, die zwischen 3 und 5 Sekunden nach Erscheinen der Antwortskala abgegeben wurden, keine Veränderungen im Muster der Antworten ergaben. Um die Gleichartigkeit der Antwortmuster zwischen Ratings, die innerhalb von 3 Sekunden und solchen, die innerhalb von 5 Sekunden abgegeben wurden zu prüfen, wurde für die Kategorisierungsbedingungen eine zweifaktorielle Varianzanalyse mit Messwiederholungen gerechnet, bei der die drei Verarbeitungsbedingungen (spontan, unbewusst, bewusst) den zwischen den Versuchspersonen variierten Gruppierungsfaktor (Kategorisierung vs. Intervention) und die auf individueller Ebene zusammengefassten Fälle (die Fall-Cluster) den Messwiederholungsfaktor darstellten. Es ergaben sich die gleichen Ergebnismuster für beide Zeitintervalle. Für die Kategorisierungsbedingungen ergibt sich jeweils ein Haupteffekt für die präsentierten Fallcluster (3-Sekunden-Intervall: $F(4$, 212) $=57,44, \quad p<0,001, \quad M S E=18727 ;$ 5-Sekunden-Intervall: $F(4,260)=79,17, \quad p<0,001$, $M S E=26478$ ), während die Variation der Verarbeitungsbedingung (3-Sekunden-Intervall: $F(2,53)=2,23, p=0,12, M S E=1215 ; 5-S e k u n d e n-I n t e r v a l l: ~ F(2,65)=2,21, p=0,12, M S E=1290)$ sowie die Interaktion zwischen den präsentierten Fällen und den Verarbeitungsbedingungen (3-Sekunden-Intervall: $\quad F(8,212)<1, \quad p=0,66, \quad M S E=239 ; \quad$ 5-Sekunden-Intervall: $\quad F(8$, 260)=1,13, $p=0,34, M S E=377)$ keine signifikanten Effekte erbrachten. Aufgrund der Gleichartigkeit der Antwortmuster in den verschiedenen Verarbeitungsbedingungen erscheint es somit gerechtfertigt, Urteile die innerhalb von 5 Sekunden nach Erscheinen der Antwortskala abgegeben wurden in die folgenden Analysen einzubeziehen.

Da auch die Daten für Urteile, die innerhalb von 5 Sekunden abgegeben wurden noch unvollständig und damit nur bedingt für weitere Analysen geeignet sind, wurden in einem weiteren Schritt fehlende Werte (also Ratings für die eine Versuchsperson länger als 5 Sekunden benötigte) durch den Mittelwert der Ratings für den jeweiligen Fall über alle Bedingungen ersetzt. Eine weitere Varianzanalyse ergab, dass sich das Muster der Antworten hierdurch nicht änderte: Es gab weiterhin lediglich einen signifikanten Haupteffekt für die präsentierten Fall-Cluster $(F(4,296)=94,61, p<0,001, M S E=31514)$, während sowohl die Variation der Verarbeitungsbedingungen $(F(2,74)=1,87, p=0,16, M S E=1010)$ als auch die 
Interaktion zwischen Fall-Clustern und Verarbeitungsbedingungen $(F(8,296)<1, p=0,73$, $M S E=217$ ) keine signifikanten Veränderungen nach sich zog. Somit dienen innerhalb von 5 Sekunden abgegebene Urteile mit durch das Mittel des jeweiligen Falles über die drei Verarbeitungsbedingungen ersetzten fehlenden Werte als Grundlage für die weiteren Analysen.

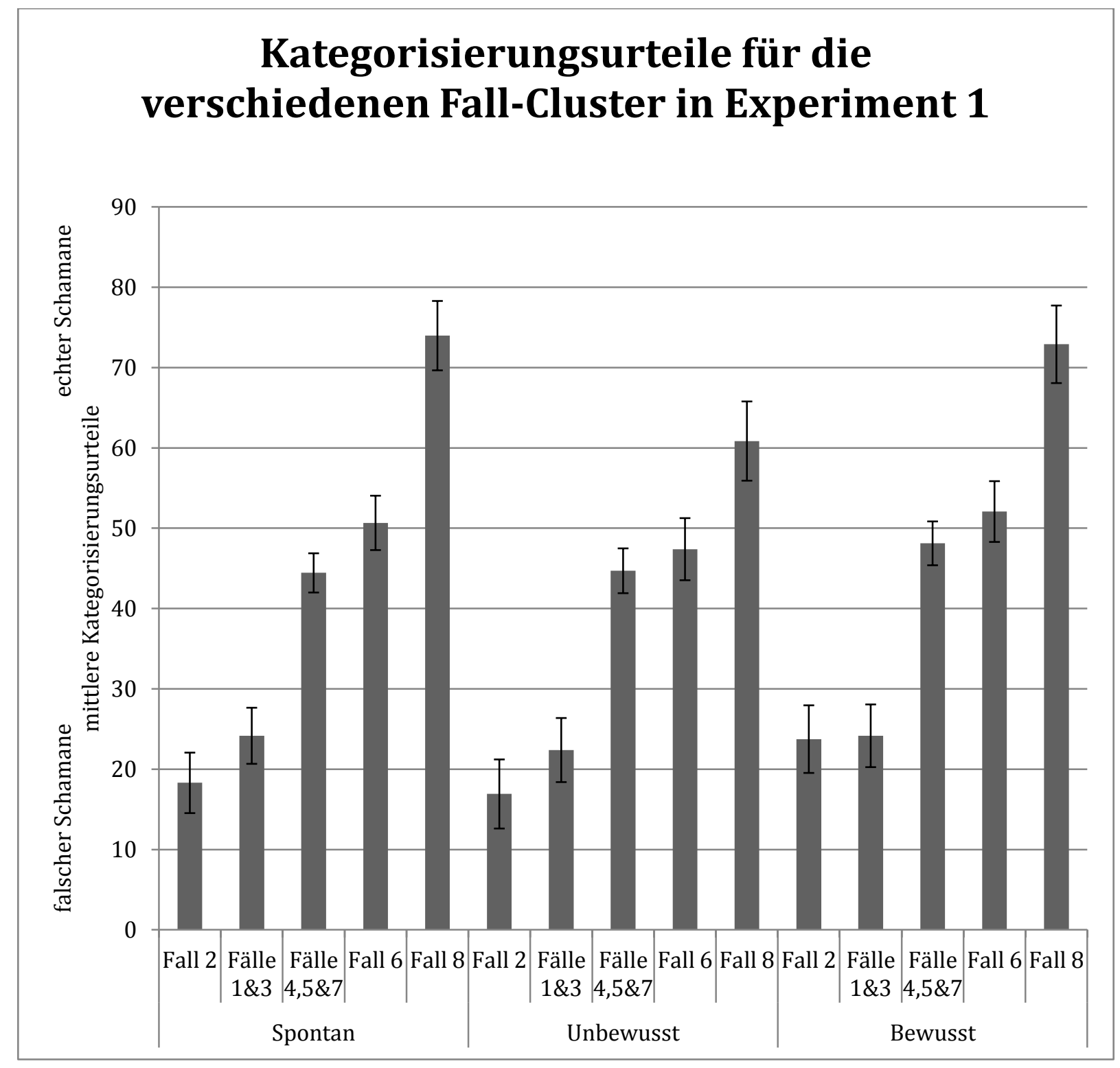

Abbildung 8: Graphische Darstellung der mittleren Kategorisierungsurteile (und Standardfehler) für die einzelnen Fall-Cluster in den verschiedenen Verarbeitungsbedingungen von Experiment 1.

Abbildung 8 zeigt eine graphische Darstellung der Kategorisierungsurteile für die einzelnen Fall-Cluster in den verschiedenen Verarbeitungsbedingungen von Experiment 1. Aus dieser Abbildung wird ersichtlich, dass die Versuchspersonen die Fall-Cluster in allen Verarbeitungsbedingungen ähnlich beurteilten. Darüber hinaus zeigt Abbildung 8, dass die Ratings für die Fall-Cluster mit gleicher Anzahl von für einen echten Schamanen 
charakteristischen Variablenausprägungen in allen drei Verarbeitungsbedingungen jeweils ähnlich hoch ausfielen. Weitere statistische Analysen auf der Basis der bereits vorgestellten Varianzanalyse sollten testen, ob die Versuchspersonen in den verschiedenen Verarbeitungsbedingungen bei ihren Kategorisierungsurteilen den kausalen Status der präsentierten Variablenausprägungen berücksichtigten. Hierzu wurden Kontraste zwischen den verschiedenen, unterschiedliche erwartete Kategorisierungsurteile repräsentierenden, Fall-Clustern gerechnet (vgl. Abschnitt 4.2.3.4).

Tabelle 1: Kontraste zum Testen der Vorhersage der kausalen Statushypothese für die Bedingungen mit Kategorisierungsaufgabe in Experiment 1. Die in die Kontraste eingehenden Ratings werden als Einschätzungen interpretiert, ob es sich bei den jeweiligen Fällen um einen echten Schamanen handelt.

\begin{tabular}{|c|c|c|c|c|}
\hline Kontrast & Alle & Spontan & Unbewusst & Bewusst \\
\hline (I) Fall 2 vs. Fälle $1 \& 3$ & $t_{(76)}=1,61, p=0,11$ & $t_{(29)}=1,51, p=0,13$ & $t_{(22)}=1,24, p=0,22$ & $t_{(23)}=0,10, p=0,92$ \\
\hline (II) Fälle $1 \& 3$ vs. Fälle $4,5 \& 7$ & $t_{(76)}=9,75, p<0,001$ & $t_{(29)}=5,60, p<0,001$ & $t_{(22)}=5,40, p<0,001$ & $t_{(23)}=5,92, p<0,001$ \\
\hline (III) Fälle 4,5\&7 vs. Fall 6 & $t_{(76)}=1,85, p=0,07$ & $t_{(29)}=1,69, p=0,10$ & $t_{(22)}=0,64, p=0,53$ & $t_{(23)}=0,96, p=0,34$ \\
\hline (IV) Fall 6 vs. Fall 8 & $t_{(76)}=5,76, p<0,001$ & $t_{(29)}=4,40, p<0,001$ & $t_{(22)}=2,22, p<0,05$ & $t_{(23)}=3,51, p<0,001$ \\
\hline
\end{tabular}

Die Ergebnisse der Berechnungen sowohl über alle Bedingungen als auch innerhalb der einzelnen Bedingungen finden sich in Tabelle 1. Bei Betrachtung der Ergebnisse fällt auf, dass die Versuchspersonen nicht zwischen den Fällen 1, 2 und 3 differenzierten (Kontrast (I) in Tabelle 1). Ebenso konnte kein Unterschied zwischen den Fällen 4, 5, 6 und 7 (Kontrast (III)) festgestellt werden. Analysiert man die Ratings für die einzelnen Verarbeitungsbedingungen, so werden dort ebenfalls ausschließlich die Kontraste (II) und (IV) signifikant. Es lässt sich somit ein Muster erkennen, demzufolge ausschließlich diejenigen Kontraste zu signifikanten Ergebnissen führen, bei denen Fall-Cluster mit unterschiedlicher Anzahl von für einen echten Schamanen charakteristischen Variablenausprägungen miteinander verglichen wurden. Bei Kontrast (II) weisen die Fälle 1 und 3 jeweils zwei Charakteristika echter Schamanen auf, während die Fälle 4, 5 und 7 vier charakteristische Eigenschaften echter Schamanen beinhalten. Ähnlich verhält es sich bei Kontrast (IV): Fall 6 weist vier, Fall 8 hingegen sechs Charakteristika echter Schamanen auf. Bei den Kontrasten (I) und (III) werden hingegen Fälle mit jeweils gleicher Anzahl an Charakteristika echter Schamanen miteinander verglichen ((I): zwei; (III): vier). Die Ergebnisse der Analyse legen somit nahe, dass die Versuchspersonen entgegen Hypothese 1 
ihr Urteil nicht auf Grundlage des kausalen Status der vorhandenen Variablen gefällt haben. Dasselbe gilt für die kausale Kohärenz der präsentierten Fälle (s. Anhang B). Es scheint so, als ob die Probanden ihr Urteil vor allem auf dem Abzählen charakteristischer Variablenausprägungen (Tallying) basiert haben.

Diese Vermutung wurde mit einer weiteren Analyse getestet, bei der Kontraste zwischen Fällen mit unterschiedlicher Anzahl an für echte Schamanen charakteristischen Variablenausprägungen miteinander verglichen wurden. Hierzu wurden zunächst neue FallCluster gebildet, in denen Fälle mit gleicher Anzahl an für einen echten Schamanen charakteristischen Variablenausprägungen zusammengefasst wurden (Fälle 1 bis 3; Fälle 4 bis 7; Fall 8). Im Anschluss wurden zur Überprüfung der Gleichartigkeit der Antwortmuster Varianzanalysen über die neuen Fall-Cluster gerechnet, die für alle drei Kriterien (Antworten, die innerhalb von 3 Sekunden abgegeben wurden; Antworten, die innerhalb von 5 Sekunden abgegeben wurden; Antworten, die innerhalb von 5 Sekunden abgegeben wurden und bei denen fehlende Werte ersetzt wurden) vergleichbare Antwortmuster ergaben: Einen Haupteffekt für Fall-Cluster (3 Sekunden: $F(2,128)=101,82, p<0,001, M S E=37342 ; 5$ Sekunden: $F(2,140)=120,52, p<0,001, M S E=40475$; 5 Sekunden $\&$ fehlende Werte ersetzt: $F(2,148)=131,85, p<0,001, M S E=42336)$, sowie eine nicht signifikante Interaktion zwischen Verarbeitungsbedingungen und Fall-Clustern (3 Sekunden: $F(4,128)<1, p=0,55, M S E=279 ; 5$ Sekunden: $F(4,140)=1,01, p=0,40, M S E=339 ; 5$ Sekunden \& fehlende Werte ersetzt: $F(4$, 148)=1,00, $p=0,41, M S E=322$ ). Im Hinblick auf die Variation der Verarbeitungsbedingung ergab sich kein Effekt für das 3 Sekunden Intervall $(F(2,64)=2,14, p=0,13, M S E=715)$ während sich für das 5 Sekunden Intervall $(F(2,70)=2,48, p=0,09, M S E=903)$ und das 5 Sekunden Intervall mit ersetzten fehlenden Werten $(F(2,74)=2,33, p=0,09, M S E=848)$ marginal signifikante Effekte ergaben. Da das Antwortmuster aber gleich war, sind diese Unterschiede wohl auf die höhere Zahl an Messwerten zurückzuführen. Daher werden die Urteile, die innerhalb von 5 Sekunden nach Erscheinen der Antwortskala abgegeben und bei denen die fehlenden Werte durch den über alle Verarbeitungsbedingungen gebildeten Durchschnitt für den jeweiligen Fall-Cluster ersetzt wurden für die weiteren Analysen herangezogen. Hierbei wurden Kontraste zwischen den Fall-Clustern gerechnet, deren Ergebnisse für die verschiedenen Verarbeitungsbedingungen in Tabelle 2 dargestellt sind. 
Tabelle 2: Kontraste zum Testen der Vorhersage der Tallying-Hypothese für die Bedingungen mit Kategorisierungsaufgabe in Experiment 1. Die in die Kontraste eingehenden Ratings werden als Einschätzungen interpretiert, ob es sich bei den jeweiligen Fällen um einen echten Schamanen handelt.

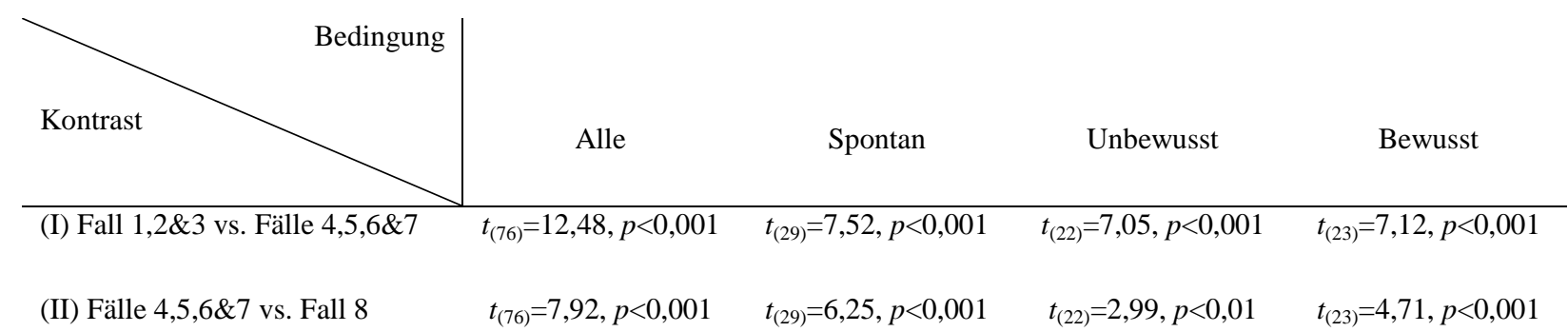

Eine graphische Darstellung der die Kontraste konstituierenden durchschnittlichen Ratings für die drei Fall-Cluster in den drei Verarbeitungsbedingungen findet sich in Abbildung 9. Es zeigt sich, dass die beiden Kontraste, die aus der Tallying-Hypothese abgeleitet wurden, in allen Verarbeitungsbedingungen signifikant sind.

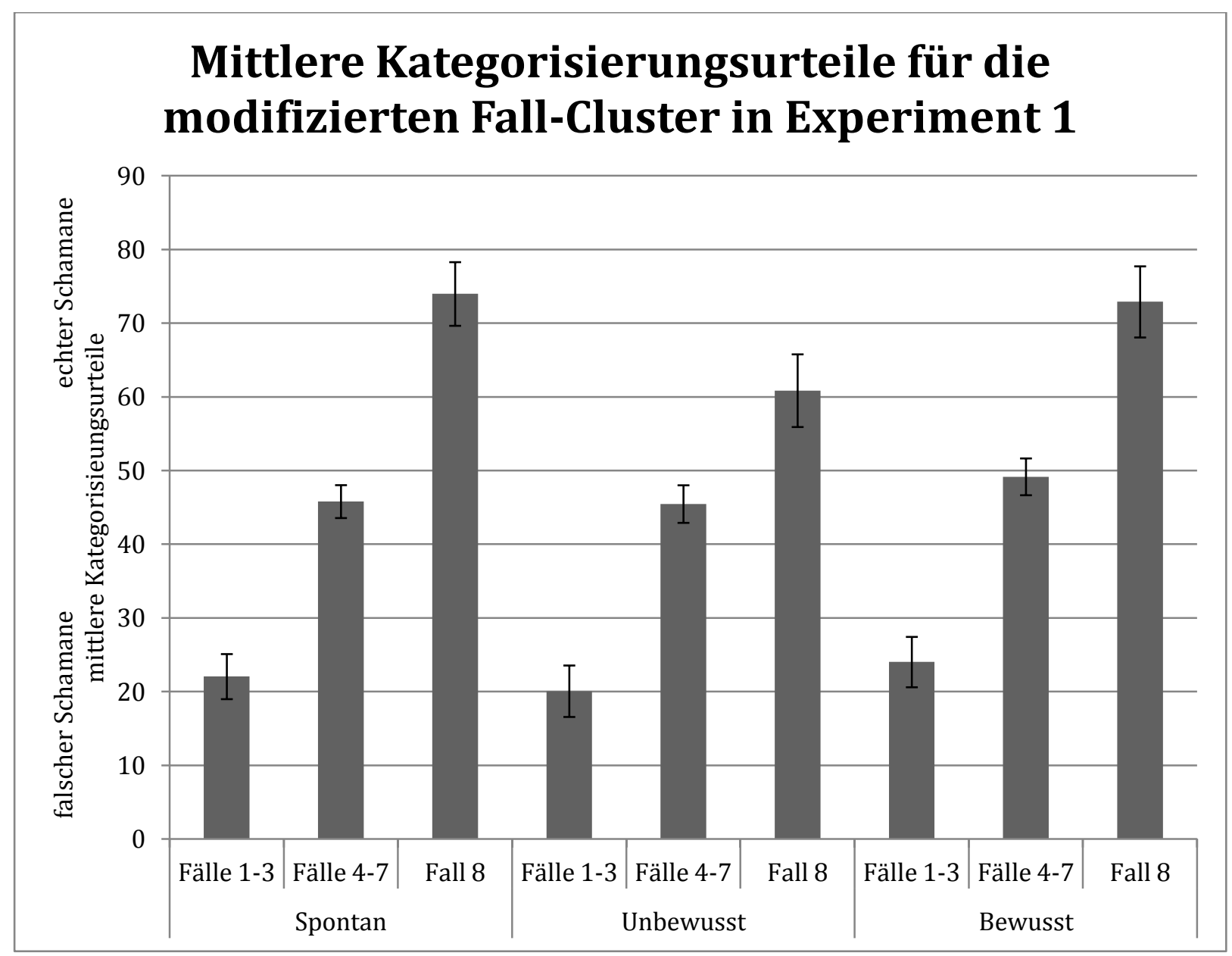

Abbildung 9: Darstellung der nach erwarteten Kategorisierungsurteilen (bezogen auf die Kategorisierung eines Falles als echter Schamane) aggregierten Kategorisierungsurteile (Fall-Cluster) in den verschiedenen Verarbeitungsbedingungen von Experiment 1. Die Fehlerindikatoren repräsentieren die jeweiligen Standardfehler. 
Die Differenz in den Ratings zwischen den unterschiedlichen Fall-Clustern unterschied sich kaum zwischen den verschiedenen Verarbeitungsbedingungen (vgl. Tabelle 3). Dies war auch dann der Fall, wenn die Differenzen der Ratings für die beiden Clustern, für die besonders extreme Urteile vorhergesagt wurden, über die Verarbeitungsbedingungen miteinander verglichen wurden. Insgesamt können diese Ergebnisse als Evidenz dafür angesehen werden, dass die Versuchspersonen ihre Urteile in erster Linie an der Anzahl der vorhandenen für einen echten Schamanen charakteristischen Variablenausprägungen orientieren. Dies geschah unabhängig von der Verarbeitungsbedingung. Da das instruierte Kausalwissen für die Bearbeitung der Kategorisierungsaufgabe somit nicht notwendig war, kann auch keine Aussage über die Gültigkeit der Hypothese getroffen werden, dass bei Kategorisierungsaufgaben keine bedeutenden Vorteile von Typ 2- gegenüber Typ 1Verarbeitungsprozessen bestehen.

Tabelle 3: Kontraste für die Vergleiche der Differenzen von Ratings für die verschiedenen Fall-Cluster zwischen den Verarbeitungsbedingungen

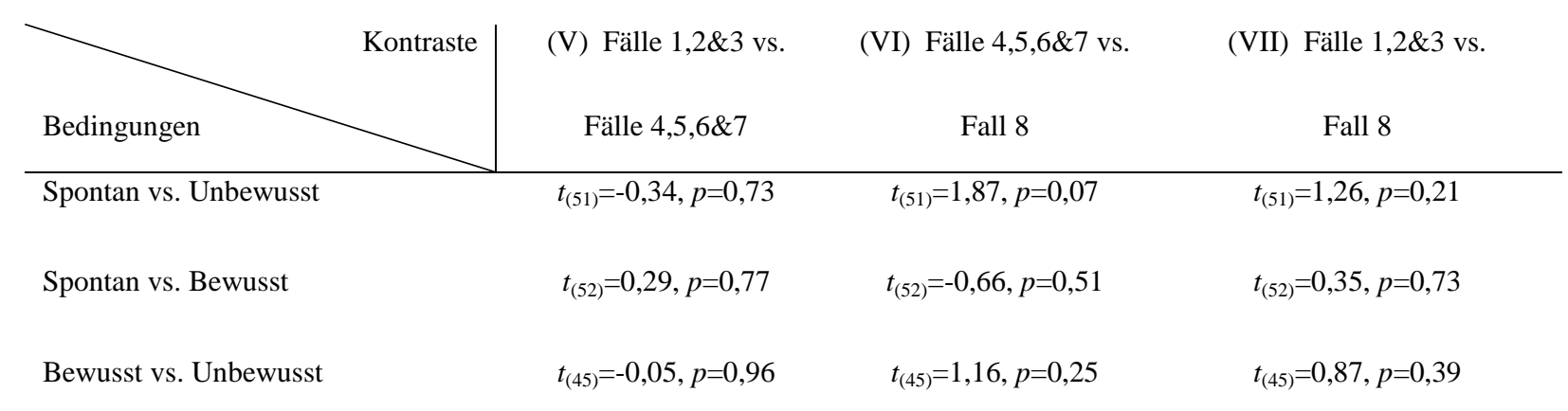

\subsubsection{Interventionsurteile}

Bei der Bewertung der verschiedenen Fälle im Hinblick auf eine zu wählende Intervention sollten sich auf der Basis des instruierten Kausalsystems in vier von acht Fällen eindeutige Präferenzen für eine der beiden möglichen Interventionen ergeben. Da in den Fällen mit Präferenz für eine bestimmte Intervention durch diese unterschiedliche viele Variablen beeinflusst werden können ergeben sich insgesamt 5 Fall-Cluster mit unterschiedlichen erwarteten Ratings (Fall1; Fall 4; Fall 2,6,7,8; Fall 5; Fall 3), für die auf Ebene der Versuchspersonen Mittelwerte gebildet wurden. Auf Grundlage dieser Werte für die fünf Fall-Cluster wurde dann die Gleichartigkeit der Antwortmuster bei 3- bzw. 5sekündigen Antwortintervallen analog zum Vorgehen bei Kategorisierungsaufgaben mittels zweifaktorieller Varianzanalyse mit Messwiederholungen geprüft. In den Interventionsbedingungen zeigte sich ein signifikanter Haupteffekt für die Fall-Cluster (3- 
Sekunden-Intervall: $\quad F(4,168)=11,46, \quad p<0,001, \quad M S E=8462 ; \quad$ 5-Sekunden-Intervall: $F(4,228)=17,33, p<0,001, M S E=13196)$ während auch hier sowohl die Variation der Verarbeitungsbedingung Effekt (3-Sekunden-Intervall: $F(2,42)<1, p=0,83, M S E=162,4$; 5Sekunden-Intervall: $F(2,57)<1, p=0,43, M S E=801)$ als auch die Interaktion zwischen den Fall-Clustern und den Verarbeitungsbedingungen (3-Sekunden-Intervall: $F(8,168)=1,26, p=0,27, M S E=929 ; 5-S e k u n d e n-I n t e r v a l l: ~ F(8,228)=1,45, p=0,18, M S E=1104)$ keine signifikanten Effekte erbrachten. Ersetzen der fehlenden Werte mit den Mittelwerten des jeweiligen Fall-Clusters über alle Verarbeitungsbedingungen erbrachte keine grundlegende Veränderung im Muster der Antworten (Fall-Cluster: $F(4,292)=21,52$, $p<0,001, M S E=15574$; Verarbeitungsbedingungen: $F(2,73)<1 ; p=0,50, M S E=570$; Interaktion zwischen Fall-Clustern und Verarbeitungsbedingung: $(F(8,292)=1,70, p=0,10, M S E=1228)$.

Tabelle 4: Kontraste zwischen den verschiedenen Fall-Clustern in den Interventionsbedingungen von Experiment 1

\begin{tabular}{|c|c|c|c|c|}
\hline Kontrast & Alle & Spontan & Unbewusst & Bewusst \\
\hline (I) Fall 1 vs. Fall 4 & $t_{(75)}=-1,88, p=0,06$ & $t_{(30)}=-1,72, p=0,09$ & $t_{(21)}=0,04, p=0,97$ & $t_{(22)}=-1,70, p=0,09$ \\
\hline (II) Fall 4 vs. Fälle $2,6,7 \& 8$ & $t_{(75)}=2,02, p<0,001$ & $t_{(30)}=3,53, p<0,001$ & $t_{(21)}=1,88, p=0,06$ & $t_{(22)}=3,42, p<0,01$ \\
\hline (III) Fälle 2,6,7\&8 vs. Fall 5 & $t_{(75)}=4,84, p<0,001$ & $t_{(30)}=2,20, p<0,05$ & $t_{(21)}=2,45, p<0,05$ & $t_{(22)}=3,69, p=0,001$ \\
\hline (IV) Fall 5 vs. Fall 3 & $t_{(75)}=-1,00, p=0,32$ & $t_{(30)}=-1,08, p<0,28$ & $t_{(21)}=-1,57, p=0,12$ & $t_{(22)}=0,86, p=0,39$ \\
\hline
\end{tabular}

Zur Testung der Hypothese wurden aufbauend auf der zuletzt berichteten Varianzanalyse Kontraste zwischen den verschiedenen Fall-Clustern gerechnet (s. Tabelle 4). Die Analyse der Kontraste zeigt deutlich, dass die Versuchspersonen nicht zwischen Fällen mit gleicher zu präferierender Intervention differenzierten (Kontraste (I) und (IV) in Tabelle 4). Die marginal signifikante Differenzierungen für Kontrast (I) in der spontanen Bedingung, sowie der Bedingung bewussten Nachdenkens, weisen sogar in die im Sinne der Vorhersage falsche Richtung, was anhand der in den Fällen 1 und 4 präsentierten Variablenausprägungen nicht ohne Weiteres erklärbar ist. Die Differenzierungsleistung für Kontrast (IV) war in diesen Bedingungen eindeutig nicht signifikant. Die einzigen signifikanten Kontraste in den beiden Bedingungen waren diejenigen, bei denen die beteiligten Fälle qualitativ unterschiedliche Aussagen hinsichtlich der zu präferierenden Intervention machten (Kontrast (II) und (III)). Dies gilt auch für die Bedingung unbewussten Denkens, bei der Kontrast (II) jedoch lediglich marginal signifikant war. 


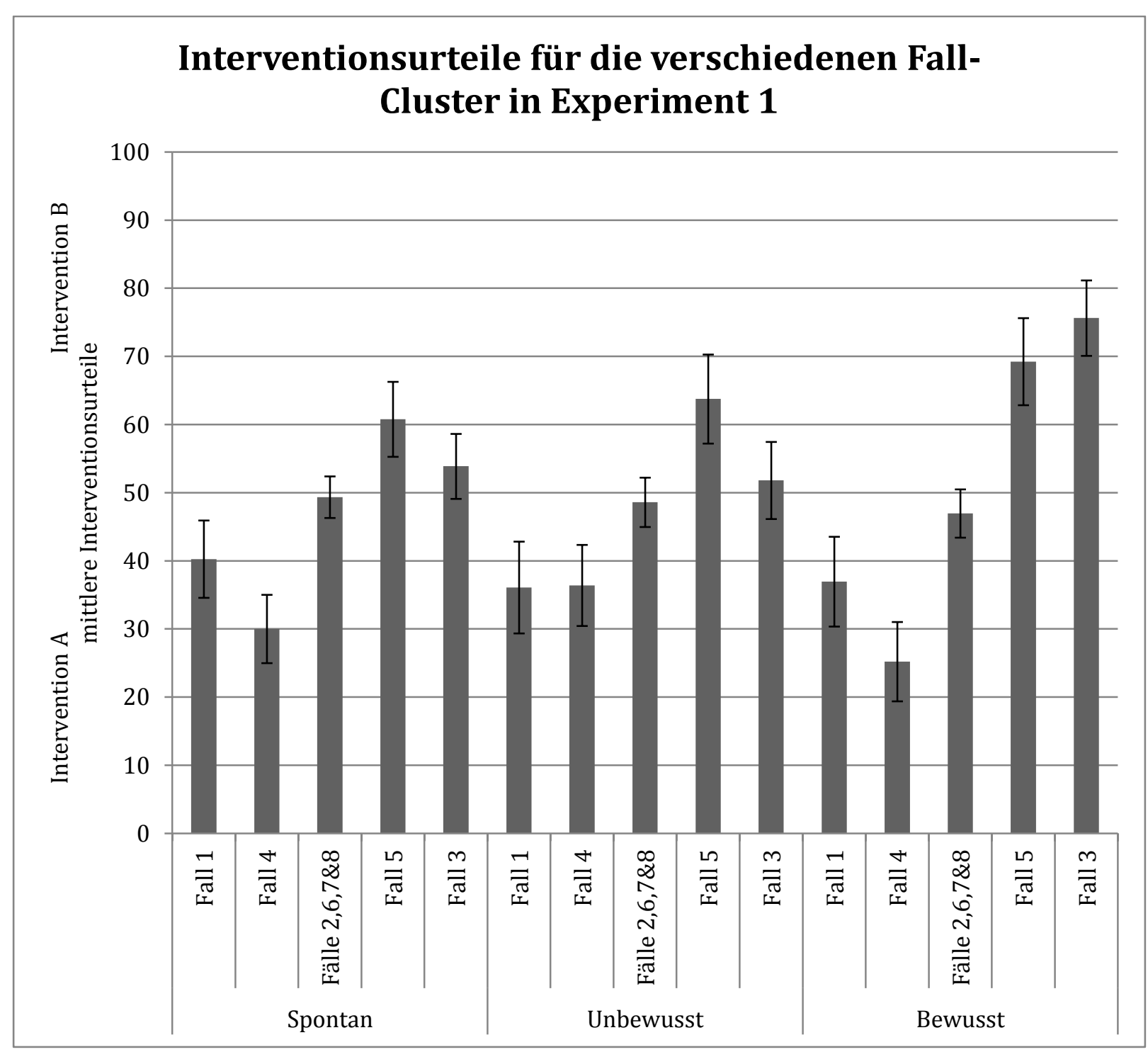

Abbildung 10: Graphische Darstellung der Interventionsurteile (und Standardfehler) in den einzelnen Verarbeitungsbedingungen von Experiment 1. Die Darstellung entspricht den in Abbildung 4 vorgestellten Fällen gemäß Balancierungsbedingung 1.

Wie auch aus Abbildung 10 ersichtlich waren die Ratings für die Fälle 1 und 4, sowie 3 und 5 in einigen Bedingungen sogar den Erwartungen in ihrer Tendenz entgegengesetzt. Auf Grundlage dieser Ergebnisse erscheint es gerechtfertigt, Abstand von der ursprünglichen Vorhersage zu nehmen. Es kann jedoch weiter davon ausgegangen werden, dass die Versuchspersonen ihre Interventionsurteile auf dem instruierten Kausalsystem basieren ohne jedoch feinere Unterschiede zwischen den Fällen mit gleicher Präferenz für eine bestimmte Intervention zu berücksichtigen. Für diese Überlegung spricht auch der Umstand, dass die in den Fällen 1 und 3 im Vergleich zu den Fällen 4 und 5 zusätzlich beeinflussbare Variable den geringsten kausalen Status im gesamten System aufweist. Daher sollte der Einfluss dieser Variablen auf das Interventionsurteil relativ gering sein. 
Zur weiteren Analyse wurden daher die Fälle auf individueller Ebene nach zu präferierender Intervention in drei verschiedenen Clustern neu zusammengefasst (Fälle 1\&4, Fälle 2,6,7\&8; Fälle 3\&5). Varianzanalysen ergaben keine grundlegenden Unterschiede in den Mustern für Antworten, die innerhalb von 3 (Fall-Cluster: $F(2,134)=36,83, p<0,001$, $M S E=19339$; Verarbeitungsbedingungen: $F(2,67)<1, p=0,43, M S E=486$; Interaktion zwischen Fall-Cluster und Verarbeitungsbedingungen: $F(4,134)=1,42, p=0,23, M S E=743)$ bzw. 5 Sekunden (Fall-Cluster: $F(2,146)=28,61, p<0,001, M S E=15151$; Verarbeitungsbedingungen: $F(2,73)<1, \quad p=0,55, \quad M S E=277$; Interaktion zwischen Fall-Cluster und Verarbeitungsbedingungen: $F(4,146)=2,06, p=0,09, M S E=1093)$ abgegeben wurden. Die Daten auf der Basis des 5-Sekunden Intervalls enthalten zudem keine fehlenden Werte und dienen daher als Basis der folgenden Kontrastanalyse (s. Tabelle 5 für die Kontraste und Abbildung 11 für eine graphische Darstellung).

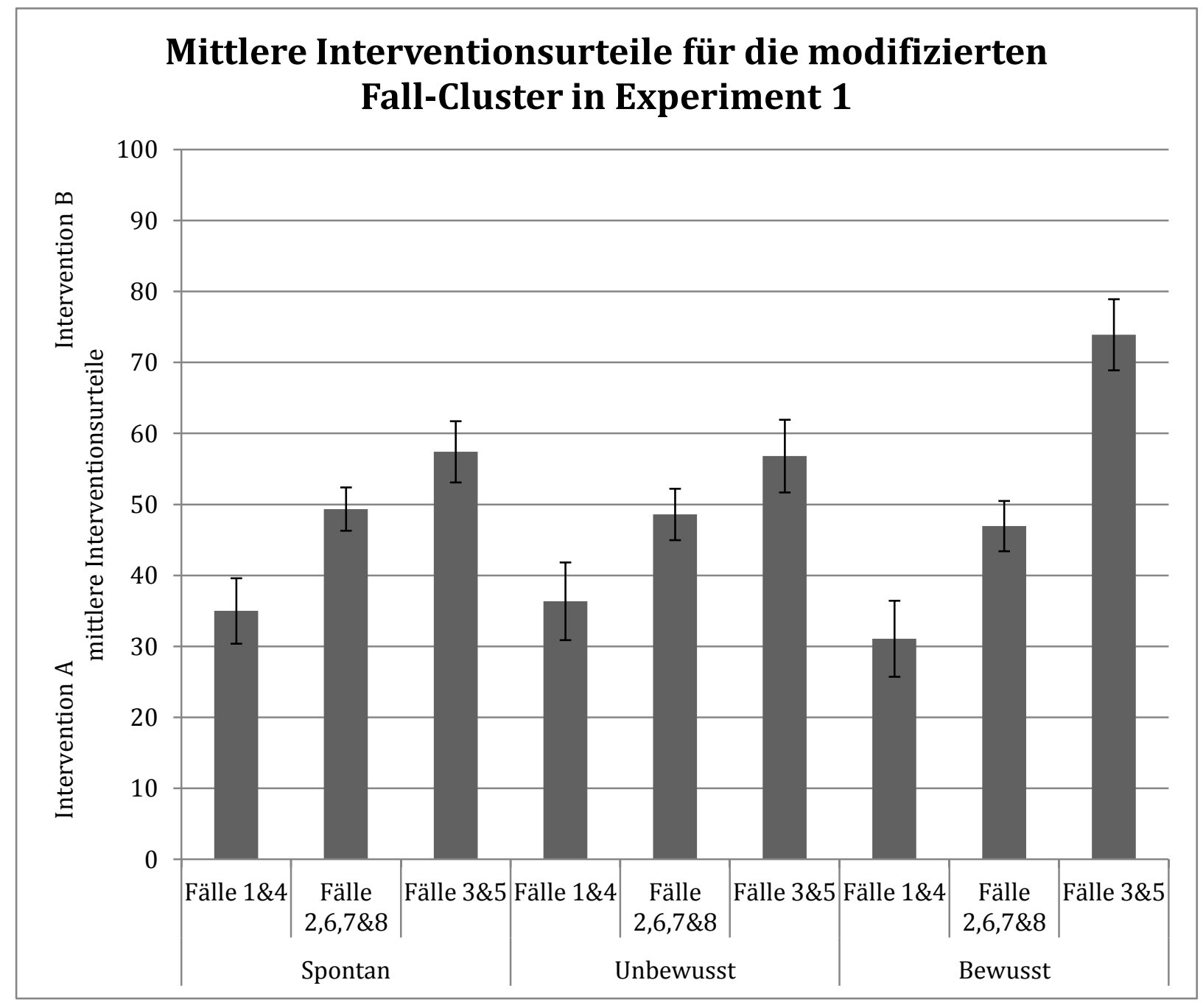

Abbildung 11: Darstellung der $\mathrm{zu}$ verschiedenen Fall-Clustern zusammengefassten mittleren Interventionsurteile (und Standardfehler) in den verschiedenen Verarbeitungsbedingungen von Experiment 1. 
In der Betrachtung über alle Verarbeitungsbedingungen ergeben sich signifikante Unterschiede für beide getesteten Kontraste ((I)und (II) in Tabelle 5). Betrachtet man hingegen die Ratings getrennt für die verschiedenen Verarbeitungsbedingungen, so zeigt sich, dass lediglich in der Bedingung bewussten Nachdenkens beide Kontraste signifikant sind. Dieser Befund liefert einen Hinweis auf die vorhergesagte Überlegenheit des Entscheidens nach einer Phase bewussten Nachdenkens im Vergleich zu sofortigem Entscheiden beim Urteilen über Interventionen auf Grundlage kausalen Wissens.

Tabelle 5: Kontraste für Fälle mit unterschiedlichen erwarteten Ratings in den Bedingungen mit Interventionsaufgaben in Experiment 1.

\begin{tabular}{l|cccc} 
& Alle & Sedingung & Unbewusst & Bewusst \\
Kontrast & $t_{(74)}=4,08, p<0,001$ & $t_{(30)}=2,67, p<0,01$ & $t_{(21)}=1,92, p=0,06$ & $t_{(22)}=2,55, p<0,05$ \\
\hline (I) Fall $1 \& 4$ vs. Fälle $2,6,7 \& 8$ & $t_{(74)}=4,85, p<0,001$ & $t_{(30)}=1,75, p=0,08$ & $t_{(21)}=1,51, p=0,14$ & $t_{(22)}=5,05, p<0,001$
\end{tabular}

Zur weiteren Prüfung der Überlegenheitshypothese wurden die Differenzen der Ratings für Fälle mit hohem erwartetem Rating und Fällen mit niedrigem erwartetem Rating zwischen den verschiedenen Verarbeitungsbedingungen kontrastiert, da nur diese eine eindeutige Präferenz für eine der beiden möglichen Interventionen besaßen. Je größer die Differenz zwischen den Ratings für die Fälle 1 und 4 (niedriges erwartetes Rating) sowie 3 und 5 (hohes erwartetes Rating) ist, desto stärker unterscheiden die Versuchspersonen zwischen den auf Grundlage des instruierten Kausalsystems zu präferierenden Interventionen. Somit sollte die vorhergesagte Überlegenheit der bewussten Verarbeitungsbedingung gegenüber der Bedingung spontanen Entscheidens bei Interventionsaufgaben in einer signifikant größeren Differenz resultieren. Dies ist auch tatsächlich der Fall ( $t_{(52 \text {, einseitig })}=1,84$, $p<0,05)$. Der Unterschied in der Differenz zwischen der Bedingung bewussten Nachdenkens und der Bedingung unbewussten Denkens, über die keine Hypothese bestand, erweist sich als marginal signifikant $\left(t_{(43)}=1,76, p=0,08\right)$. Zwischen der Bedingung sofortigen Entscheidens und der Bedingung unbewussten Denkens konnte kein Unterschied festgestellt werden $\left(t_{(51)}=0,17, p=0,86\right)$. Insgesamt zeigt sich somit, dass bei Interventionsaufgaben - entsprechend der Vorhersage - Entscheidungen nach einer Phase bewussten Nachdenkens solchen, die unmittelbar nach der Präsentation der Stimuli erfolgen, überlegen sind. 


\subsubsection{Diskussion}

Die Ergebnisse von Experiment 1 haben für die aufgestellten Hypothesen folgende Bedeutung: Hypothese 2 wurde durch die Ergebnisse der Interventionsbedingungen bestätigt, während über Hypothese 1 keine Aussage gemacht werden kann. Diese Befunde werden im Folgenden erläutert. Die Ergebnisse der Kategorisierungsaufgaben legen zunächst nahe, dass die Versuchspersonen in allen drei Verarbeitungsbedingungen in der Lage waren, auf Basis kausaler Faktoren die richtigen Entscheidungen für die präsentierten Fälle zu treffen. Eine Analyse der Ratings im Hinblick auf die durch die kausale Statushypothese gemachten Vorhersagen legte jedoch nahe, dass die Urteile der Versuchspersonen möglicherweise deshalb nicht auf instruiertem kausalem Wissen basierten, weil es möglich war, die Kategorisierungsaufgabe durch einfaches Abzählen der für einen echten (bzw. falschen) Schamanen charakteristischen Variablenausprägungen (Tallying) während der Stimuluspräsentation zu absolvieren. Bei der später erfolgenden Abfrage mussten die so gebildeten Kategorisierungsurteile lediglich wiedergegeben werden. Der Vorgang der Urteilsbildung ist unter der Tallying-Hypothese wenig komplex. Daher erscheint es prinzipiell auch möglich, dass ein durch ein solches Online-Tallying gebildetes Urteil auch nach Durchführung einer 2-back-Distraktoraufgabe korrekt wiedergegeben werden kann. Aufgrund dieser plausiblen alternativen Erklärung verlieren die Ergebnisse der Kategorisierungsaufgaben in Experiment 1 ihre Aussagekraft im Hinblick auf die Berücksichtigung zuvor erlernten Kausalwissens bei Kategorisierungsurteilen. Somit kann auch die aufgestellte Hypothese 1 nicht auf der Grundlage der erhaltenen Resultate beurteilt werden.

Die Interventionsurteile der Probanden können jedoch nicht ohne eine Berücksichtigung kausalen Wissens erklärt werden. Die Gestaltung der Testfälle stellte sicher, dass die Probanden korrekte Urteile nur auf Basis der Struktur des instruierten Kausalsystems treffen konnten. Die Ergebnisse bestätigen die aufgestellte Hypothese 2, dass bewusstes Nachdenken bei Interventionsentscheidungen zu besseren Urteilen führt als eine unmittelbar nach der Stimuluspräsentation erfolgende Entscheidung. Somit zeigt sich bei Interventionsentscheidungen eine Überlegenheit von Typ 2- gegenüber Typ 1Verarbeitungsprozessen. Darüber hinaus ergab sich auch eine tendenzielle Überlegenheit bewussten Nachdenkens gegenüber Entscheidungen, die nach Bearbeitung einer Distraktoraufgabe abgegeben wurden. Ein weiterer Indikator für die Überlegenheit bewussten Nachdenkens besteht darin, dass nur die Versuchspersonen in dieser Bedingung in der Lage 
waren, sowohl zwischen Fällen mit niedrigen vs. mittleren erwarteten Ratings, als auch zwischen Fällen mit mittleren vs. hohen erwarteten Ratings signifikant zu unterscheiden.

\subsection{Experiment 2}

\subsubsection{Ziel des Experiments}

Das nun vorzustellende zweite Experiment hatte erneut zum Ziel, die kognitiven Prozesse zu untersuchen, welche kausalen Kategorisierungs- und Interventionsentscheidungen zu Grunde liegen. Dabei sollte die Methodik im Vergleich zu Experiment 1 so verändert werden, dass ein bloßes Abzählen von Merkmalsausprägungen nicht länger hinreichend für eine erfolgreiche Bewältigung der Kategorisierungsaufgabe ist. Die Kategorisierungsaufgabe wurde so modifiziert, dass unterschiedliche Kategorisierungsurteile nur durch eine Berücksichtigung der kausalen Struktur zu erklären sind, welche die einzelnen Merkmale miteinander verbindet. Zudem wurde darauf geachtet, dass die Vorhersagen für die einzelnen Fälle auf Grundlage des kausalen Status und der kausalen Kohärenz identisch sind. Darüber hinaus wurde eine Replikation der Ergebnisse der Bedingungen mit Interventionsaufgaben aus Experiment 1 angestrebt. Eine weitere wichtige Veränderung betraf die Verarbeitungsbedingung, in der die Versuchspersonen aufgefordert wurden, bewusst über die zu beantwortende Frage nachzudenken. Während die Dauer dieser Aufgabe in der Testphase von Experiment 1 auf 2 Minuten festgelegt war und damit der Dauer der 2-back-Aufgabe entsprach, konnten die Versuchspersonen in Experiment 2 selbst wählen, wie lange sie nachdenken wollten (vgl. Payne, Samper, Bettman \& Luce,2008). Diese Veränderung wurde vorgenommen, da Menschen in Alltagssituationen ebenfalls nicht für eine bestimmte von außen festgelegte Dauer über die Lösung eines Problems nachdenken, sondern diesen Zeitraum für gewöhnlich selbst bestimmen. Zudem hatten vorherige Studien gezeigt, dass ein freie Wahl des Zeitraums bewussten Nachdenkens zu besseren Urteilen führt (Payne et al., 2008). Für die Verarbeitungsbedingungen mit Kategorisierungsaufgaben wurde gemäß der in Abschnitt 3.4 aufgestellten Hypothesen vorhergesagt, dass die Kategorisierungsurteile der Versuchspersonen in der Bedingung bewussten Nachdenkens denjenigen von Versuchspersonen in der Bedingung spontanen Entscheidens nicht überlegen sind. Für die Bedingungen mit Interventionsaufgaben wurden hingegen bessere Urteile der Versuchspersonen in der Bedingung bewussten Denkens gegenüber denen in der Bedingung spontanen Entscheidens vorhergesagt. 


\subsubsection{Stichprobe und experimentelles Design}

Die Gesamtzahl der an Experiment 2 teilnehmenden Versuchspersonen betrug 136. Die Versuchspersonen wurden zufällig auf die sechs Bedingungen des Experiments aufgeteilt. Experiment 2 hatte die Form eines 2 (Aufgabe: Kategorisierung vs. Intervention) X 3 (Verarbeitungsbedingung: Spontan vs. Unbewusst vs. Bewusst mit selbstgewählter Dauer) X 4 (präsentierte Fälle) Designs, bei dem die Art der Aufgabe, sowie die Verarbeitungsbedingungen zwischen den Versuchspersonen, die präsentierten Fälle hingegen innerhalb der Versuchspersonen variiert wurden.

\subsubsection{Ablauf und verwendetes Stimulusmaterial}

Grundsätzlich folgte Experiment 2 dem in Abschnitt 4.1 dargestellten Ablauf der im Rahmen dieser Arbeit vorgestellten Experimente. Allerdings gab es einige Besonderheiten, auf die an dieser Stelle eingegangen werden soll. Wie bereits erwähnt, wurde die Bedingung bewussten Nachdenkens aus Experiment 1 durch eine Bedingung bewussten Nachdenkens mit selbstgewählter Dauer ersetzt. In dieser Bedingung konnten die Versuchspersonen in bestimmten Grenzen selbst wählen, wie lange sie über die von ihnen zu bearbeitende Aufgabe nachdenken wollten. Diese Grenzen bestanden zum einen in einer Mindestdauer der Nachdenkphase von 10 Sekunden. Die Umsetzung dieser Einschränkung erfolgte durch eine um 10 Sekunden verzögerte und für die Versuchspersonen sichtbare Aktivierung der „Weiter"'-Schaltfläche auf dem während der Nachdenk-Phase eingeblendeten Bildschirm. Die Versuchspersonen wurden hierüber in den Instruktionen in Kenntnis gesetzt. Zudem wurden sie während der Übungsphase mit dieser Besonderheit vertraut gemacht. Die Mindestdauer sollte sicherstellen, dass die Versuchspersonen sich zumindest etwas mit der Aufgabe beschäftigen mussten und nicht unmittelbar nach Ende der Stimuluspräsentation auf die Frage antworten konnten. Damit sollte die Unterscheidbarkeit zur Bedingung des spontanen Entscheidens gewährleistet werden. Zum anderen war die maximale Dauer der Nachdenkphase auf 2 Minuten begrenzt, da dies auch die in der Bedingung unbewussten Denkens verfügbare Zeit war. Die maximal verbleibende Zeit wurde in der Bedingung bewussten Nachdenkens mit selbstgewählter Dauer ebenso wie in der Bedingung unbewussten Nachdenkens durch einen auf dem Bildschirm befindlichen Countdown angezeigt.

Nachdem die Versuchspersonen mit den verschiedenen Verarbeitungsbedingungen von Experiment 2 in der Übungsphase vertraut gemacht worden waren, wurde ihnen in der 
Lernphase das Kausalsystem mittels einer Coverstory instruiert. Die Versuchspersonen sollten sich in die Rolle eines/einer auf Reptilien spezialisierten Tierarztes/-ärztin versetzten. Ihre Aufgabe bestand in den Kategorisierungsbedingungen darin, einzelne Komodowarane hinsichtlich des Vorliegens einer bestimmten Erkrankung zu kategorisieren. Dabei sollten sie anhand der Symptomkonstellation eines Warans entscheiden, ob die sogenannte Torokrankheit bei diesem Tier vorliegt oder es an einer anderen Erkrankung leidet. Die Aufgabe der Versuchspersonen in den Bedingungen mit Interventionsaufgaben bestand darin, aufgrund der Symptomkonstellation erkrankter Warane eine von zwei sich gegenseitig ausschließenden möglichen Behandlungen zu wählen. Dabei sollte diejenige Behandlung gewählt werden, die das Potenzial besitzt, die meisten Symptome eines Warans zu beseitigen. Die Torokrankheit wurde als Kausalsystem beschrieben, in dem die verschiedenen Symptome kausal miteinander verknüpft waren. Das System bestand aus insgesamt 10 Variablen, die über 10 Kausalrelationen miteinander verknüpft waren (s. Abbildungen 12 und 13). Jede der Variablen stellte ein Symptom der Krankheit dar, das entweder vorliegen oder nicht vorliegen konnte. Durch die Konstellation der Symptome eines Warans sollte es zum einen möglich sein, die Wahrscheinlichkeit des Vorliegens der Torokrankheit zu schätzen (Kategorisierungsaufgabe). Zum anderen sollte sie die Differenzierung zwischen den beiden Behandlungsmöglichkeiten erlauben, da sich die Beseitigung eines Symptom auf die von ihm direkt oder indirekt verursachten Symptome auswirkt. Die Darstellung des Kausalsystems wurde in zwei Stufen balanciert, um Effekte der Position der Systemvariablen kontrollieren zu können.

Im Gegensatz zu Experiment 1 wurden in Experiment 2 keine expliziten Basisraten für die verschiedenen Variablen genannt. Es wurde jedoch ausdrücklich darauf hingewiesen, dass keines der Symptome für die Diagnose der Erkrankung zwingend notwendig ist. Damit sollte die Plausibilität des Auftretens unterschiedlicher Symptomkonstellationen erhöht werden. Um einen besseren Eindruck der den Versuchspersonen vermittelten Instruktionen bezüglich des Kausalsystems zu bekommen, werden diese im Folgenden wiedergegeben. Da sich die Instruktionen für Kategorisierungs- und Interventionsaufgaben in Experiment 2 stark ähneln wird auf eine getrennte Darstellung verzichtet. Am Ende eines jeden Absatzes der Darstellung der Instruktionen wird in eckigen Klammern angegeben, ob der jeweilige Abschnitt den Versuchspersonen in den Kategorisierungsbedingungen, den Interventionsbedingungen oder in beiden Bedingungen präsentiert wurde. 


\subsubsection{Instruktionen Kausalsystem Warane}

„,Stelle Dir bitte folgende Situation vor Du bist Tierarzt/-ärztin und hast dich auf Erkrankungen bei Reptilien spezialisiert. Bei mehreren Zoos übernimmst du die Behandlung jeglicher Reptilien. Einige Zoos haben in diesem Jahr über eine Stiftung gefährdete Komodowarane (Varanus komodoensis) aus Indonesien angekauft. Bis vor einigen Tagen akklimatisierten sich die Warane gut, aber seit gestern zeigen vier der Warane Krankheitssymptome. Du vermutest, dass sie an der sogenannten Torokrankheit leiden könnten, eine für die sonst so robusten Warane nicht seltene, aber sehr gefährliche Krankheit." [Kategorisierung \& Intervention]

„Deine Aufgabe als Experte wird es sein, aufgrund der Symptomenkonstellation zu entscheiden, wie wahrscheinlich es ist, dass die Torokrankheit bei dem jeweiligen Tier vorliegt, oder ob das betroffene Tier an einer anderen Erkrankung leidet. " [Kategorisierung]

„Es gibt zwei Behandlungen, die Du für verschiedene Symptome einsetzen kannst. Allerdings kann nur eine Behandlung auf einmal durchgeführt werden, da sich die Wirkung von beiden gegenseitig aufhebt. Deine Aufgabe als Experte wird es sein, aufgrund der Symptomenkonstellation zu entscheiden, welche der beiden Behandlungen effektiver ist (d.h. die meisten Symptome behebt) und deshalb als erstes durchgeführt werden soll." [Intervention]

„Bei der Torokrankheit liegt in der Regel eine erhöhte Anzahl von den ansonsten nützlichen Adeno- und Coronabakterien vor, die im Magen-Darm-Trakt der Tiere siedeln. Diese Bakterien rufen nun gefährliche Symptome hervor. Beide Arten von Bakterien zerstören dann häufig die Darmflora und verursachen ebenfalls häufig eine heftige Entzündung der Magenschleimhaut. Eine zerstörte Darmflora hat oftmals Diarrhö zur Folge. Diarrhö führt meist zu einer Dehydration des Körpers der Warane. Außerdem bekommen kranke Tiere in Folge der zerstörten Darmflora oftmals Koliken. Eine Entzündung der Magenschleimhaut ruft häufig Erbrechen hervor. Durch das Erbrechen wird häufig die empfindliche Speiseröhre der Warane nachhaltig geschädigt. Außerdem führt eine Entzündung der Magenschleimhaut oft zu starken Schmerzen. Keines dieser Symptome muss zwangsläufig bei der Torokrankheit vorliegen. Häufig ist nur ein Teil der Symptome vorhanden." [Kategorisierung \& Intervention]

„Selbst wenn beispielsweise keine erhöhte Anzahl an Adeno- und/oder Coronabakterien vorliegt, kann eine Diagnose vergeben werden." [Kategorisierung]

„Eine der beiden Behandlungsarten wirkt sehr effektiv gegen eine zerstörte Darmflora. Die andere Behandlung wirkt sehr effektiv gegen eine Entzündung der Magenschleimhaut." [Intervention]

„Auf der nächsten Folie haben wir dir die möglichen Symptome und die Kausalzusammenhänge zwischen diesen noch einmal graphisch veranschaulicht, so dass du sie besser verinnerlichen kannst. Bitte präge Dir die Symptome und ihre Kausalzusammenhänge gut ein, sodass du später entscheiden kannst, ob du zuerst 
die Darmflora oder die Magenschleimhaut behandeln würdest. Drücke einfach auf ,Weiter" um dir diese anzuschauen. " [Kategorisierung \& Intervention]

Im Anschluss an die verbale Beschreibung des Kausalsystems wurde dieses mittels einer Graphik veranschaulicht. Die Graphiken waren für alle Versuchspersonen einheitlich mit der Überschrift „Mögliche Symptome und ihre Kausalzusammenhänge bei Vorliegen der Torokrankheit beim Komodowaran (Varanus komodoensis)“ versehen. Abbildung 12 zeigt die graphische Darstellung der Balancierungsbedingung 1 wie sie den Versuchspersonen in den Kategorisierungsbedingungen präsentiert wurde. Zur Generierung der zweiten Balancierungsbedingung wurden die Merkmale an der vertikalen Achse gespiegelt. Der einzige Unterschied in den Systemdarstellungen für die einzelnen Aufgabentypen bestand darin, dass in den Interventionsbedingungen die beiden möglichen Interventionen dargestellt wurden (s. Abbildung 13).

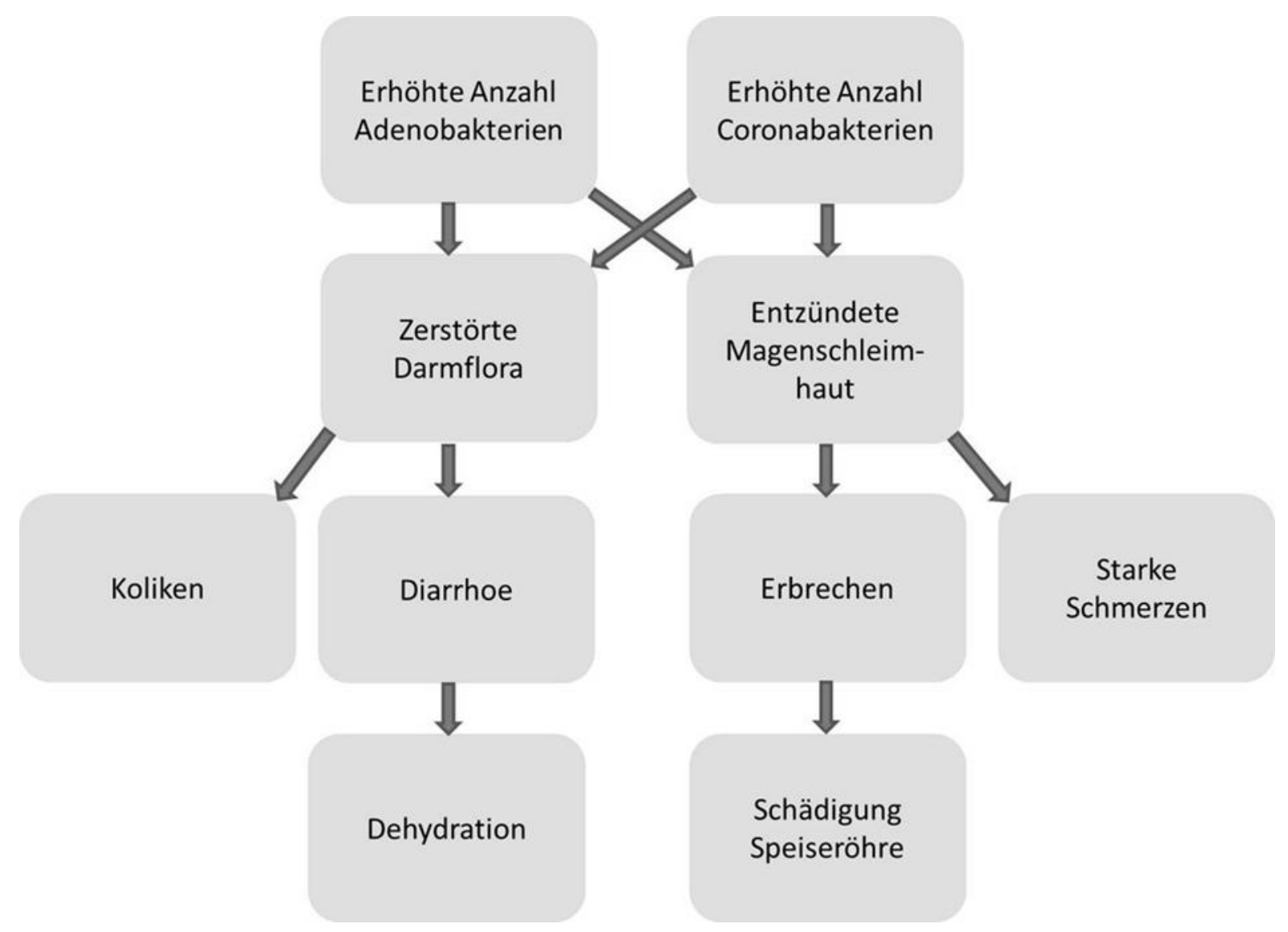

Abbildung 12: Darstellung des in Experiment 2 verwendeten Kausalsystems mit den verschiedenen Variablen in Balancierungsbedingung 1.Die Pfeile zwischen den Variablen repräsentieren Kausalrelationen und sind von der verursachenden Variablen auf den jeweiligen Effekt gerichtet. Die in dieser Abbildung dargestellten Variablenausprägungen stellen die verschiedenen Symptome der Torokrankheit dar. Die Abwesenheit einzelner Symptome wird während der Stimuluspräsentation durch das Wort „kein“ bzw. „keine“ vor dem Namen des Symptoms gekennzeichnet. 


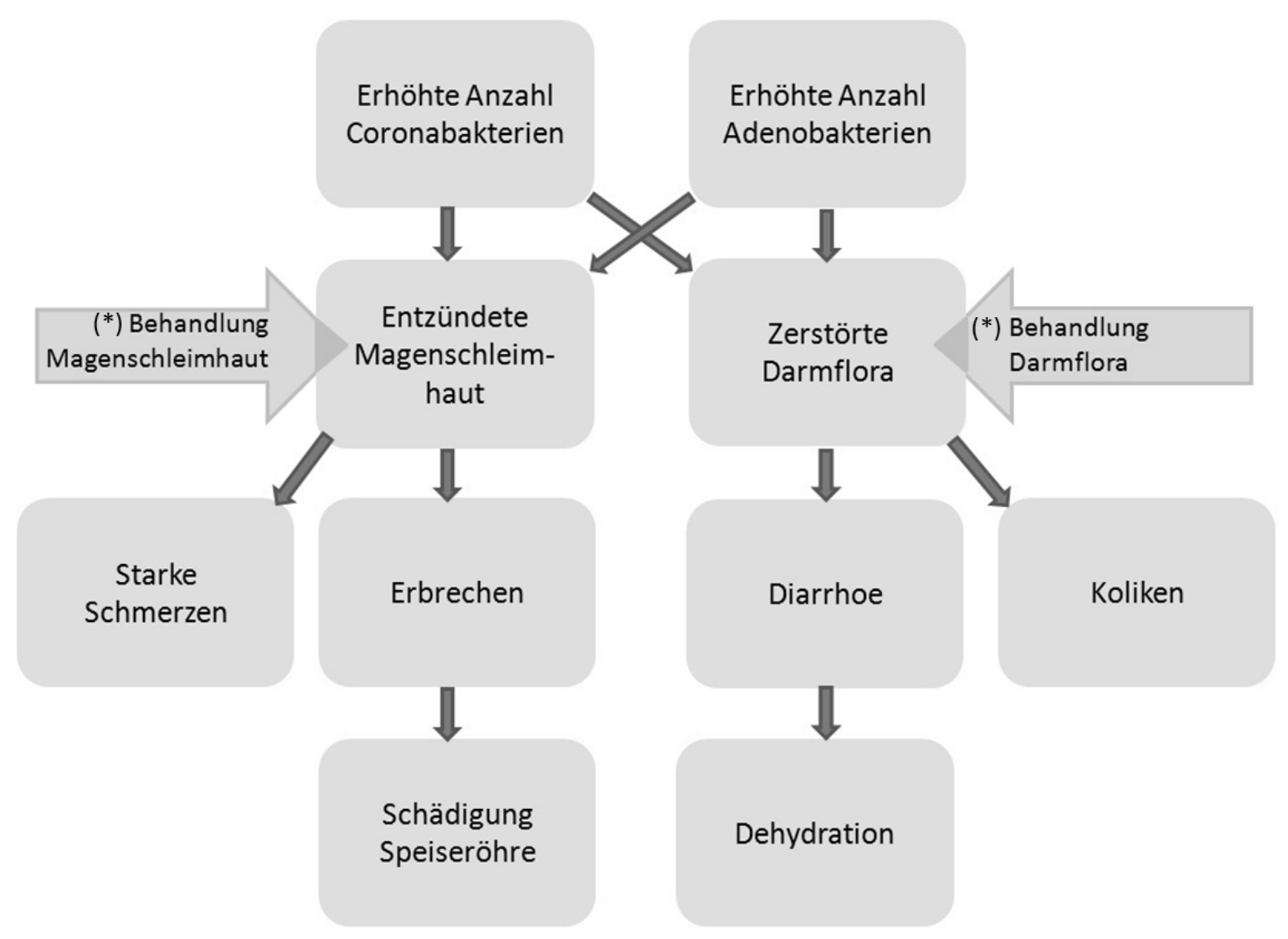

Abbildung 13: Darstellung des in Experiment 2 verwendeten Kausalsystems mit den verschiedenen Variablen in Balancierungsbedingung 2.Die Pfeile zwischen den Variablen repräsentieren Kausalrelationen und sind von der verursachenden Variablen auf den jeweiligen Effekt gerichtet. Die in dieser Abbildung dargestellten Variablenausprägungen stellen die verschiedenen Symptome der Torokrankheit dar. Die mit $(*)$ gekennzeichneten Felder repräsentieren die beiden möglichen Interventionen und wurden ausschließlich den Versuchspersonen in den Bedingungen mit Interventionsaufgaben präsentiert. Die Abwesenheit einzelner Symptome wird während der Stimuluspräsentation durch das Wort „kein“ bzw. „keine“ vor dem Namen des Symptoms gekennzeichnet.

\subsubsection{Multiple-Choice Wissenstest}

Das Wissen der Versuchspersonen bezüglich des Kausalsystems wurde mit 8 Multiple-Choice-Fragen getestet. Bei jeder Frage gab es vier Antwortalternativen, von denen eine korrekt war (s. Anhang C). Beantwortete eine Versuchsperson alle Fragen korrekt, so gelangte sie in die Testphase des Experiments. Machte sie hingegen mindestens einen Fehler, so mussten die Instruktionen mitsamt anschließendem Wissenstest erneut durchlaufen werden. Hatte eine Versuchsperson nach sechs Durchgängen nicht alle Fragen korrekt beantwortet, gelangte sie dennoch in die Testphase. Allerdings wurden ihre Daten als ungültig markiert (vgl. Abschnitt 4.1). Dasselbe geschah, wenn eine Versuchsperson länger als 30 Minuten für Übungs- und Lernphase des Experimentes benötigte. Der Grund hierfür lag in der 
Gewährleistung des reibungslosen Ablaufs der Experimente im Computerlabor, der gestört wurde, wenn einzelne Versuchspersonen für die Experimente zu viel Zeit benötigten.

\subsubsection{Testphase}

Die Testphase bestand aus vier Durchgängen mit je einem Testfall. In jedem dieser Durchgänge wurde nach dem Instruktionsbildschirm zunächst die zu beantwortende Frage für ca. 3 Sekunden präsentiert, die für die Versuchspersonen in den Kategorisierungsbedingungen folgenden Wortlaut hatte: „Hat Waran X die Torokrankheit oder eine andere Krankheit?“،, wobei $\mathrm{X}$ die Nummer des $\mathrm{zu}$ beurteilenden Warans, die der Nummer des Versuchsdurchganges entsprach, darstellte. Die Versuchspersonen antworteten nach Ablauf ihrer jeweiligen Verarbeitungsaufgabe durch Mausklick auf eine von elf Schaltflächen, die eine elfstufige Skala repräsentierten und mit in 1er Schritten zunehmenden Zahlenwerten von -5 bis +5 beschriftet waren. Der linke Pol der Skale (-5) war mit „sicher Torokrankheit“, der rechte Pol (+5) mit „sicher andere Krankheit“ gekennzeichnet. Für die Versuchspersonen in den Interventionsbedingungen lautete die Frage: „Welche Behandlung soll Waran X bekommen?“, wobei X ebenfalls dem Testdurchgang entsprechende Nummer des zu beurteilenden Warans darstellte. Die Pole der von -5 bis +5 reichenden elfstufigen Antwortskala waren gemäß dem in den Abbildungen 12 und 13 dargestellten System gekennzeichnet. In der Balancierungsbedingung 1 war dies für den linken Pol (-5) „sicher Behandlung Darmflora“ und für den rechten Pol $(+5)$,sicher Behandlung Magenschleimhaut“. In Balancierungsbedingung 2 wurde die Bezeichnung umgekehrt. Es ist $\mathrm{zu}$ beachten, dass in den Bedingungen mit Kategorisierungsaufgaben keine Balancierung der Skala erfolgte, da diese hier in keinem Zusammenhang mit der Position der Variablen in der jeweils präsentierten graphischen Darstellung des Kausalsystems stand.

Im Anschluss an die Präsentation der Frage erfolgte eine Einblendung der Bezeichnung des im aktuellen Durchgang zu beurteilenden Waran (,Waran 1“, „Waran 2“ usw.) für ca. 3 Sekunden. Unmittelbar im Anschluss wurden die Variablenausprägungen für den entsprechenden Waran einzeln in der Mitte des Bildschirms für ca. 1,5 Sekunden präsentiert. Die verschiedenen Symptome der Torokrankheit konnten entweder an- oder abwesend sein. Abwesende Symptome wurden durch das Wort „kein“ bzw. „keine“ vor dem Namen des Symptoms gekennzeichnet (z.B. „kein Erbrechen“, „keine Dehydration“). Die Reihenfolge der präsentierten Variablenausprägungen wurde für jeden Fall randomisiert. Ebenso wurde die Reihenfolge der präsentierten Fälle randomisiert. Unmittelbar nach der Präsentation der letzten Variablenausprägung eines Falles wurden in den Bedingungen 
unbewussten Denkens und den Bedingungen bewussten Denkens mit selbstgewählter Dauer die jeweiligen Aufgaben automatisch präsentiert. Im Anschluss an diese Aufgaben (in den Bedingungen sofortigen Entscheidens: unmittelbar nach der Stimuluspräsentation) wurde die zu beantwortende Frage abermals für ca. 3 Sekunden präsentiert. Die abermalige Präsentation der Frage sollte die Versuchspersonen auf das baldige Erscheinen der Antwortskala vorbereiten, um zu verhindern, dass eine große Zahl von Zeitüberschreitungen erfolgt. Nach Ablauf dieser Zeitspanne wurde zusätzlich zur Frage die elfstufige Antwortskala eingeblendet, auf der die Versuchspersonen ihre Einschätzung bezüglich der präsentierten Frage innerhalb von 3 Sekunden abzugeben hatten. Benötigten sie länger als 3 Sekunden erschien unmittelbar nach Abgabe der Einschätzung ein Hinweisfenster mit der Aufforderung beim nächsten Mal schneller zu antworten. Der Zeitpunkt des Erscheinens des Hinweisfensters wurde gewählt, um die in Experiment 1 vorhandene Möglichkeit des Nachdenkens über die Einschätzung unmittelbar vor deren Abfrage zu verhindern.

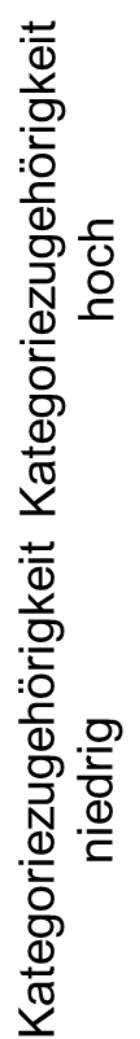

Intervention A effektiver
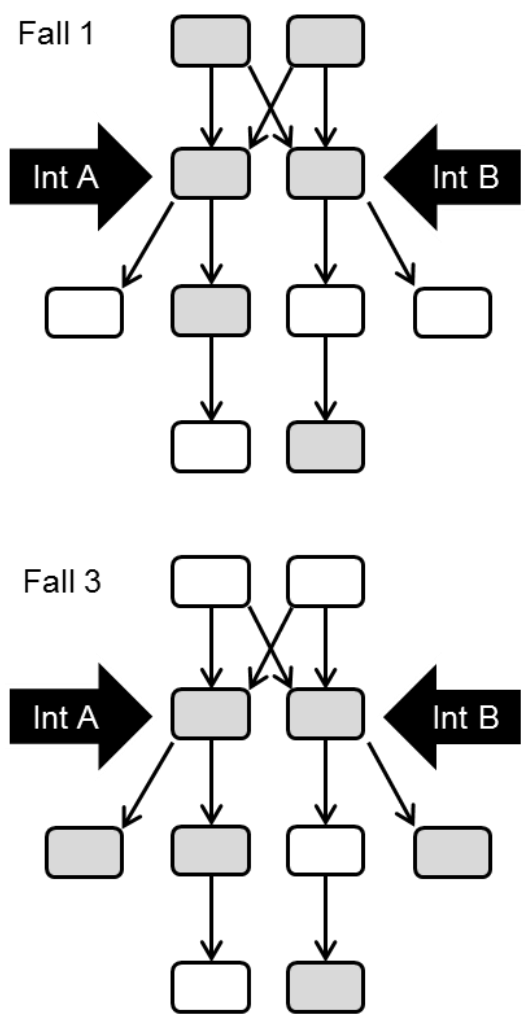
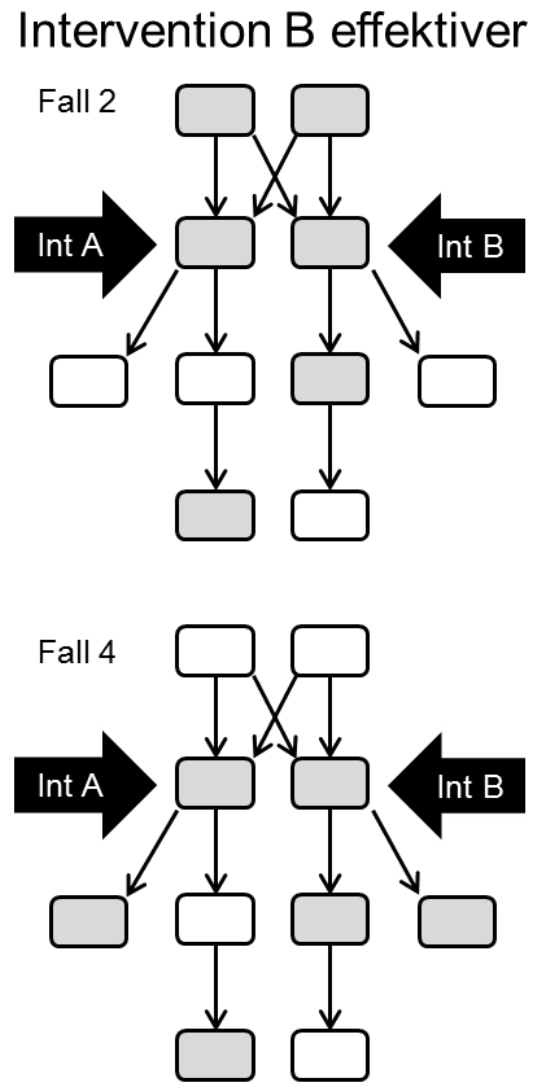

Abbildung 14: Schematische Darstellung der in Experiment 2 verwendeten Fälle inkl. der Vorhersagen für beide Aufgabenarten gemäß Balancierungsbedingung 1. Die Felder repräsentieren die Variablen des Kausalsystems, die Pfeile die kausalen Verbindungen zwischen den Variablen, wobei die Pfeilspitze auf den Effekt in der jeweiligen Relation weist. Grau gefärbte Felder repräsentieren die im jeweiligen Fall anwesenden Symptome. Für die Interventionsbedingungen sind jeweils die beiden möglichen Interventionen („Int A“ und „Int B“) als zusätzliche schwarz gefärbte pfeilförmige Felder dargestellt. „Int A“ entspricht dabei der Behandlung der Darmflora, „Int B“ der Behandlung der Magenschleimhaut. Diese Felder haben für die Kategorisierungsbedingungen keine Relevanz. Die Vorhersagen für die einzelnen Fälle können den Achsenbeschriftungen entnommen werden (Y-Achse: Kategorisierung; X-Achse: Intervention). 
Die in Experiment 2 verwendeten Fälle sind in Abbildung 14 schematisch dargestellt. Wie in Experiment 1 wurden auch in Experiment 2 identische Fälle für die beiden Aufgabenarten verwendet. Daher sind die Symptome, auf die die beiden Interventionen in den Interventionsbedingungen wirken in allen vier Fällen anwesend. Eine weitere Besonderheit der verwendeten Fälle besteht in der gleichen Anzahl vorhandener Symptome. Wenn die Probanden wie in Experiment 1 nur die Anzahl der Symptome zählen, sollten sie daher zu gleichen Kategorisierungsurteilen für alle Fälle kommen.

Die Vorhersagen für die einzelnen Fälle in den verschiedenen Bedingungen gemäß Hypothese 1 sind ebenfalls in Abbildung 14 dargestellt: In den Kategorisierungsbedingungen sollten die Fälle 1 und 2 am ehesten als Warane, die unter der Torokrankheit leiden kategorisiert werden. Bei diesen Fällen besitzen die anwesenden Symptome einen höheren kausalen Status als bei den Fällen 3 und 4. Daneben weisen die Fälle 1 und 2 auch eine höhere kausale Kohärenz als die Fälle 3 und 4 auf. Für die Entscheidungen in den Bedingungen mit Interventionsaufgabe ist gemäß Hypothese 2 die Anzahl der Symptome ausschlaggebend, die durch eine Intervention direkt oder indirekt beseitigt werden können. Hier zeigt sich ein Vorteil in Bezug auf die Anzahl der potenziell behebbaren Symptome zugunsten von Intervention A (Behandlung der Darmflora) gegenüber Intervention B (Behandlung der Magenschleimhaut) im Verhältnis 2:1 für Fall 1 und 3:2 für Fall 3. Hingegen ist Intervention B in Fall 2 mit einem Verhältnis von 2:1 und 3:2 in Fall 4 gegenüber Intervention A im Vorteil. Die probabilistische Natur der Kausalrelationen im verwendeten Kausalsystem hat aufgrund der Balancierung der Variablenausprägung in den einzelnen Fällen keine Bedeutung für die zu präferierende Intervention.

\subsubsection{Ergebnisse}

Daten von 111 der ursprünglichen 136 Versuchspersonen gingen in die Analyse ein. Bei zwei Versuchspersonen konnten aufgrund technischer Probleme außer den demographischen keine weiteren Daten aufgezeichnet werden, 22 Versuchspersonen scheiterten am Multiple-Choice-Wissenstest über das verwendete Kausalsystem und eine Versuchsperson brach das Experiment vorzeitig ab. 81 der 111 für die folgenden Analysen relevanten Versuchspersonen waren Frauen (Anteil: 73,0\%) und 50 Studierende der Psychologie (45,0\%). Damit bildeten Psychologiestundenten wie bereits in Experiment 1 die größte Gruppe von Versuchspersonen. In die Analyse der Kategorisierungsaufgaben gingen die Daten von 57 Versuchspersonen ein, von denen sich jeweils 19 in den drei Verarbeitungsbedingungen befanden. Von den 54 Versuchspersonen, die verwertbare Daten 
in den Interventionsbedingungen lieferten, kamen jeweils 18 aus den drei Verarbeitungsbedingungen. Im Durchschnitt benötigten die Versuchspersonen ca. 13 Minuten in den Bedingungen sofortigen Entscheidens (Kategorisierung: ca. 14 Minuten; Intervention: ca. 12 Minuten), ca. 25 Minuten in den Bedingungen unbewussten Denkens (Kategorisierung: ca. 25 Minuten; Intervention: ca. 26 Minuten) und ca. 18 Minuten in den Bedingungen des bewussten Nachdenkens mit selbstgewählter Dauer (Kategorisierung: ca. 17 Minuten; Intervention: ca. 20 Minuten). Die durchschnittliche Zeit, die die Versuchspersonen in den Bedingungen bewussten Nachdenkens mit selbstgewählter Dauer zum Überlegen benötigten betrug 18,4 Sekunden (Kategorisierung: 17,8 Sekunden; Intervention: 18,9 Sekunden). Lediglich eine Versuchsperson in der Interventionsbedingung benötigte für die Beurteilung eines Falles die maximal erlaubte Nachdenk-Zeit von 2 Minuten.

Für die nachfolgenden Analysen wurden auf der Ebene der Versuchsperson zunächst die Ratings für die Fälle mit gleichem erwarteten Rating gemittelt (vgl. Abschnitt 4.3.3.3). In den Bedingungen mit Kategorisierungsaufgaben wurden die Ratings der Fälle 1 und 2 (hohe erwartete Kategorisierungsurteile) sowie 3 und 4 (niedrige erwartete Kategorisierungsurteile) auf diese Weise zu Fall-Clustern zusammengefasst. Für die Bedingungen mit Interventionsurteilen wurden die Urteile nicht zusammengefasst, da auch zwischen den Fällen mit Präferenz für die gleiche Intervention Unterschiede bezüglich der Anzahl beeinflussbaren Variablen bestehen. Wie bereits in Experiment 1 wurden auch in Experiment 2 Urteile, die innerhalb von fünf Sekunden nach Erscheinen der Antwortskala abgegeben wurden, in die Analyse einbezogen, sofern sich das allgemeine Antwortmuster nicht von demjenigen unterschied, das auf Urteilen basierte, die innerhalb von 3 Sekunden abgegeben wurden.

\subsubsection{Multiple-Choice Wissenstest}

Insgesamt konnte der Multiple-Choice Wissenstest von 22 der 134 Versuchspersonen (16\%) nicht erfolgreich absolviert werden. Diejenigen Versuchspersonen, die alle Fragen korrekt beantworten konnten, benötigten hierfür durchschnittlich 2,28 Durchgänge. Die durchschnittlich benötigten Durchgänge in den verschiedenen Bedingungen sind in Abbildung 15 dargestellt. Weder die Art der Aufgabe $(F(1,106)=1,18, p=0,28, M S E=2)$ noch die Interaktion zwischen Aufgabenart und Verarbeitungsbedingung $(F(2,106)<1, \quad p=0,61$, $M S E=1$ ) hatte einen Einfluss auf die Anzahl der Durchgänge beim Mulitple-ChoiceWissenstest. Der Einfluss der Verarbeitungsbedingung $(F(2,106)=2,64, p=0,08, M S E=5)$ war marginal signifikant. Dieser Unterschied ist jedoch inhaltlich nicht erklärbar, da die Versuchspersonen in den Verarbeitungsbedingungen einer Aufgabenart jeweils identische 
Instruktionen bezüglich des Kausalsystems erhielten und auch identische Multiple-ChoiceFragen zu beantworten hatten.

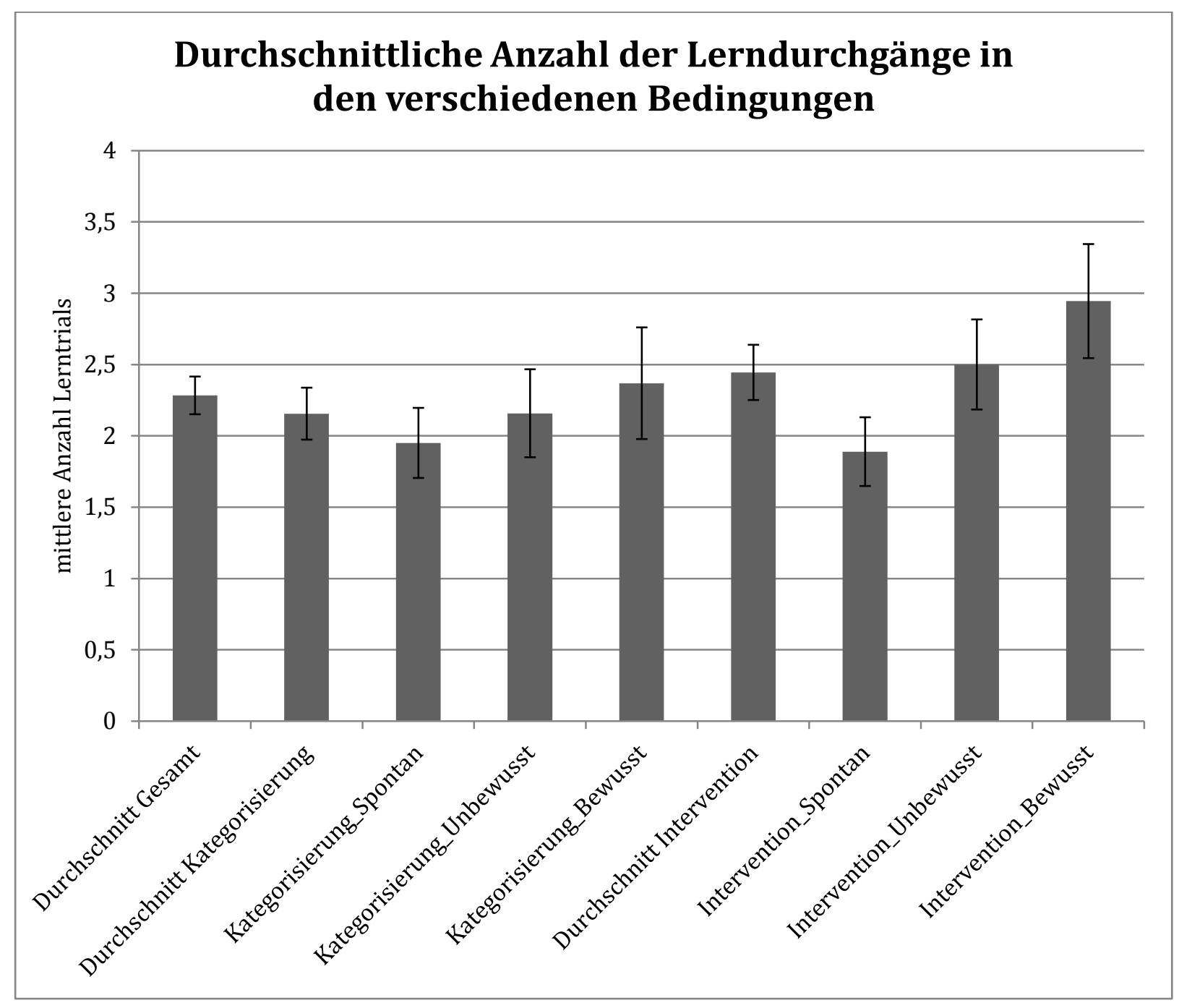

Abbildung 15: Durchschnittliche Anzahl der von den Versuchspersonen in Experiment 2 benötigten Durchgänge (und zugehörige Standardfehler) beim Multiple-Choice Wissenstest.

\subsubsection{Kategorisierungsurteile}

Die mittels zweifaktorieller Varianzananlysen mit Messwiederholungen vorgenommene Prüfung der Gleichartigkeit der Antwortmuster für Urteile, die nach 3 bzw. 5 Sekunden abgegeben wurden ergab keine bedeutsamen Unterschiede. Für beide Intervalle wurde ein signifikanter Effekt der Fall-Cluster (3 Sekunden: $F(1,52)=8,55, p<0,01$, $M S E=3803$; 5 Sekunden: $F(1,54)=8,97, p<0,01, M S E=3763)$ festgestellt, während die Variation der Verarbeitungsbedingungen (3 Sekunden: $F(2,52)<1, p=0,39, M S E=413 ; 5$ Sekunden: $F(2,54)<1, p=0,52, M S E=281)$, sowie die Interaktion von Fall-Cluster und Verarbeitungsbedingungen (3 Sekunden: $F(2,52)=1,36, p=0,26, M S E=606 ; 5$ Sekunden: $F(2,54)<1, p=0,56, M S E=245)$ keine signifikanten Effekte hervorbrachten. Aufgrund der 
Gleichartigkeit der Antwortmuster und dem Nicht-Vorhandensein fehlender Daten bilden die innerhalb von 5 Sekunden abgegebenen Urteile die Basis für die weitere Analyse.

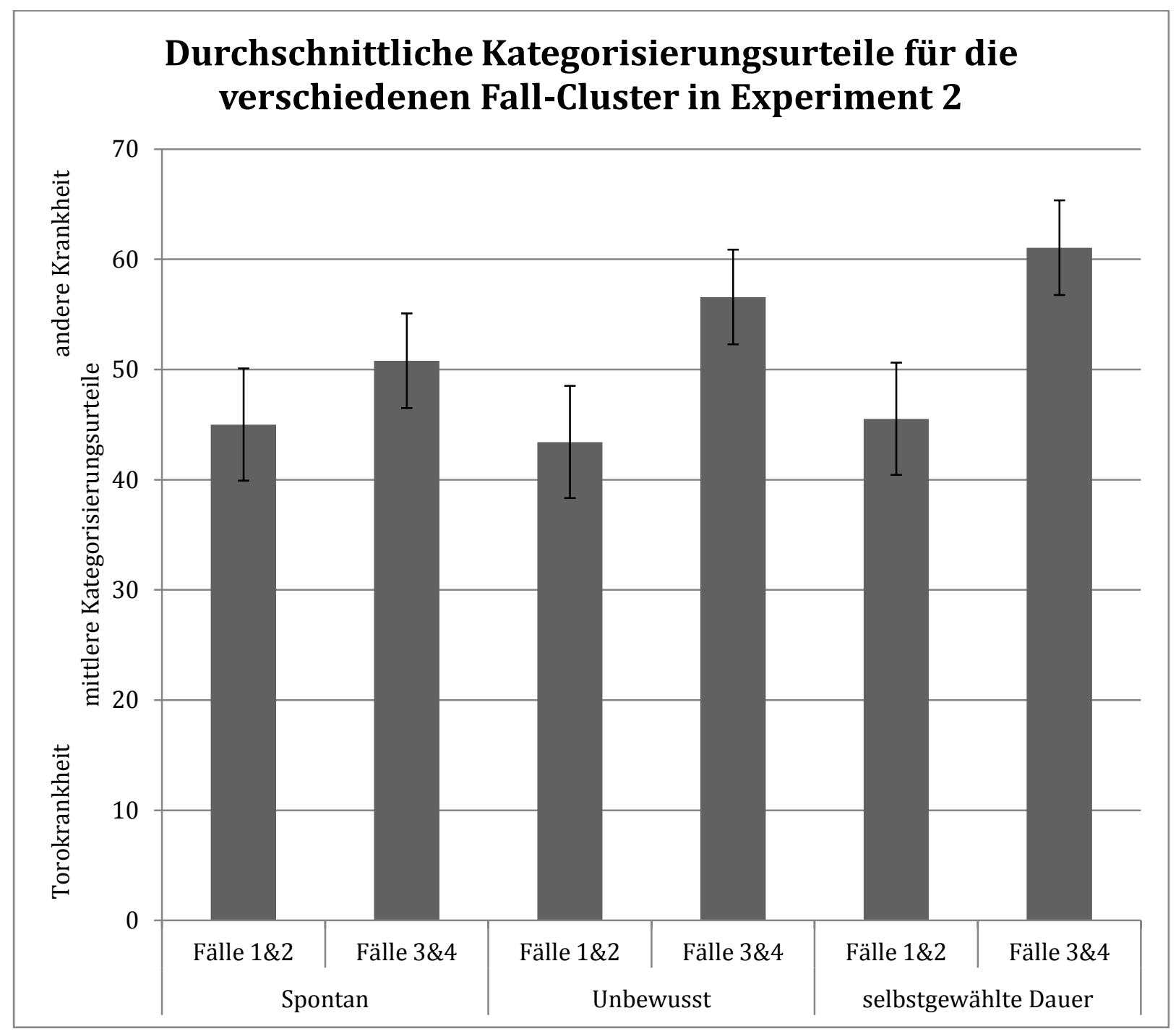

Abbildung 16: Durchschnittliche Kategorisierungsurteile (und Standardfehler) in den verschiedenen Verarbeitungsbedingungen von Experiment 2. Die dargestellten Fall-Cluster stellen auf individueller Ebene gemittelte Ratings für Fälle für die gleiche Kategorisierungsurteile erwartet wurden dar und basieren auf Urteilen, die innerhalb von 5 Sekunden nach Erscheinen der Antwortskala abgegeben wurden.

Die auf der individuellen Ebene für Fälle mit gleichem erwarteten Kategorisierungsurteil gemittelten Ratings in den verschiedenen Verarbeitungsbedingungen von Experiment 2 sind in Abbildung 16 graphisch dargestellt. Aus der Abbildung ist bereits die Tendenz ablesbar, dass die Versuchspersonen in der Bedingung unbewussten Nachdenkens, sowie der Bedingung bewussten Nachdenkens mit selbstgewählter Dauer eher in der Lage waren, zwischen den beiden Fall-Clustern zu unterscheiden. Dieser Eindruck wurde durch eine Kontrastanalyse bestätigt. Der Unterschied in den Urteilen zwischen Fällen mit niedrigen und solchen mit hohen erwarteten Ratings ist über alle Bedingungen signifikant $\left(t_{(56)}=2,99, p<0,01\right)$. Betrachtet man jedoch die Unterschiede getrennt für die einzelnen 
Verarbeitungsbedingungen, so kann nur in der Bedingung bewussten Nachdenkens mit selbstgewählter Dauer $\left(t_{(18)}=2,34, p<0,05\right)$ ein signifikanter Unterschied festgestellt werden. In der Bedingung unbewussten Denkens ist ein marginal signifikanter Unterschied vorhanden $\left(t_{(18)}=1,98, p=0,05\right)$, während in der Bedingung spontanen Entscheidens kein Unterschied festgestellt werden kann $\left(t_{(18)}=0,87, \quad p<0,39\right)$. Die Differenzierungsleistung der Versuchspersonen in der Bedingung bewussten Nachdenkens mit selbstgewählter Dauer war jedoch nicht signifikant besser als in der Bedingung spontanen Entscheidens $\left(t_{(36)}=1,04\right.$, $p=0,30$ ). Somit konnte keine Überlegenheit des bewussten Nachdenkens gegenüber dem sofortigen Entscheiden bei den Kategorisierungsaufgaben in Experiment 2 festgestellt werden. Auch die übrigen Vergleiche der Differenzen zwischen den Verarbeitungsbedingungen ergaben keine signifikanten Unterschiede (unbewusstes Denken vs. spontanes Entscheiden: $t_{(36)}=0,78, p=0,44$; bewusstes Denken mit selbstgewählter Dauer vs. unbewusstes Denken: $\left(t_{(36)}=0,25, p<0,80\right)$.

\subsubsection{Interventionsurteile}

Für die Verarbeitungsbedingungen mit Interventionsaufgaben in Experiment 2 konnten keinerlei signifikante Effekte für die präsentierten Fälle bzw. die Verarbeitungsbedingungen festgestellt werden. Dieses Resultat ergab sich unabhängig davon, ob Urteile analysiert wurden, die innerhalb von 3 Sekunden nach Erscheinen der Antwortskala abgegeben wurden (Variation der Verarbeitungsbedingung: $F(2,15)<1, p=0,92$, $M S E=59$; präsentierte Fälle: $F(3,45)<1, \quad p=0,74, \quad M S E=325 ; \quad$ Interaktion zwischen Verarbeitungsbedingungen und präsentierten Fällen: $F(6,45)<1, p=0,96, M S E=181)$, die innerhalb von 5 Sekunden nach Erscheinen der Skala abgegeben wurden (Variation der Verarbeitungsbedingung: $F(2,36)<1, \quad p=0,99, M S E=10$; präsentierte Fälle: $F(3,108)<1$, $p=0,91, M S E=128$; Interaktion zwischen Verarbeitungsbedingungen und präsentierten Fällen: $F(6,108)=1,24, p=0,29, M S E=852)$ oder die auf Antworten basierten, die innerhalb von 5 Sekunden abgegeben wurden und bei denen die fehlenden Werte durch die über alle Bedingungen gemittelten Ratings für den jeweiligen Fall ersetzt wurden (Variation der Verarbeitungsbedingung: $F(2,51)<1, p=0,71, M S E=219$; präsentierte Fälle: $F(3,153)<1$, $p=0,86, M S E=171$; Interaktion zwischen Verarbeitungsbedingungen und präsentierten Fällen: $F(6,153)<1, p=0,57, M S E=534)$. Das Muster der Antworten änderte sich auch dann nicht, wenn die Urteile für die Fälle mit Präferenz für eine bestimmte Intervention auf individueller Ebene gemittelt und neue Analysen auf Grundlage der so entstandenen neuen Datenbasis durchgeführt wurden (z.B. für Antworten, die innerhalb von 5 Sekunden abgegeben wurden 
und bei denen es keine fehlenden Werte gab: Variation der Verarbeitungsbedingung: $F(2,51)<1, p=0,49, M S E=258$; Fall-Cluster: $F(1,51)<1, p=0,54, M S E=223$; Interaktion zwischen Verarbeitungsbedingungen und Fall-Clustern: $F(2,51)<1, p=0,81, M S E=123)$. Aufgrund der eindeutigen Ergebnisse der durchgeführten Varianzanalysen erscheint eine weitere Kontrastanalyse überflüssig. Offensichtlich waren die Versuchspersonen also nicht in der Lage, zwischen den präsentierten Fällen hinsichtlich der zu präferierenden Intervention zu differenzieren, wie auch aus der graphischen Darstellung der mittleren Urteile für die vier präsentierten Fälle in den drei Verarbeitungsbedingungen in Abbildung 17 ersichtlich ist. Die Abbildung basiert auf Urteilen, die 5 Sekunden nach Erscheinen der Antwortskala abgegeben wurden. Urteile, bei denen die Versuchspersonen länger brauchten wurden durch über alle Verarbeitungsbedingungen gemittelte Ratings für den entsprechenden Fall ersetzt.

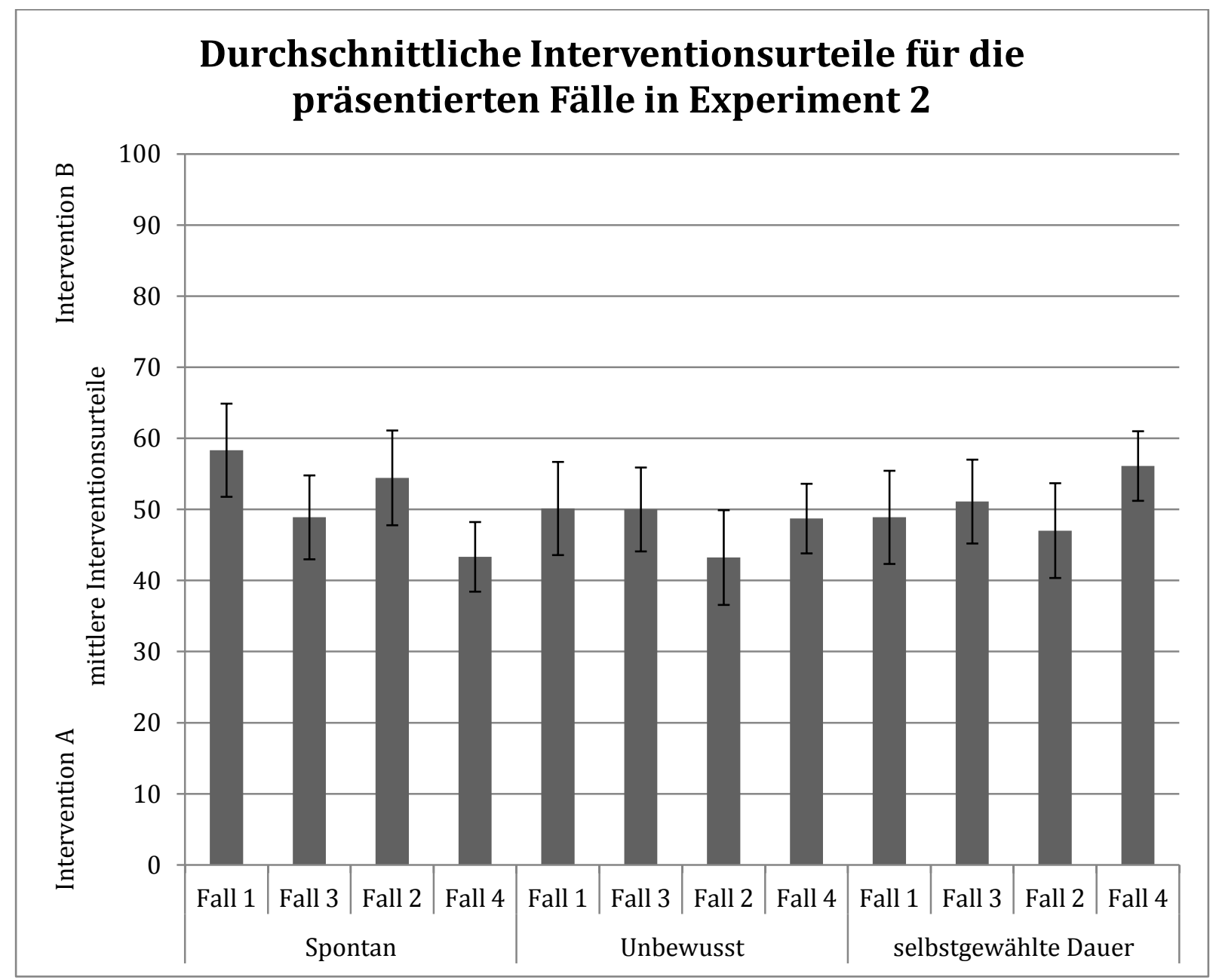

Abbildung 17: Durchschnittliche Interventionsurteile (und Standardfehler) in den verschiedenen Verarbeitungsbedingungen von Experiment 2. Die dargestellten Fälle basieren auf Urteilen, die innerhalb von 5 Sekunden nach Erscheinen der Antwortskala abgegeben wurden und bei denen fehlende Werte durch den über alle Bedingungen gebildeten Mittelwert für den jeweiligen Fall ersetzt wurden. Die Fälle sind für die einzelnen Verarbeitungsbedingungen nach erwarteten Urteilen sortiert. 


\subsubsection{Diskussion}

Die beiden aufgestellten Hypothesen sind auf Grundlage der Ergebnisse von Experiment 2 nur schwer $\mathrm{zu}$ bewerten. Da es den Versuchspersonen in den Interventionsbedingungen in keiner Verarbeitungsbedingung gelang, zwischen den Fällen mit unterschiedlichem erwarteten Interventionsurteil $\mathrm{zu}$ differenzieren, kann auch keine Überlegenheit von Typ 2-Verarbeitungsprozessen im Sinne von Hypothese 2 angenommen werden. In den Kategorisierungsbedingungen konnte ebenfalls keine Überlegenheit des bewussten Denkens gegenüber dem spontanen Entscheiden festgestellt werden, was prinzipiell für die Gültigkeit von Hypothese 1 spricht. Allerdings unterschieden die Versuchspersonen in der Bedingung spontanen Entscheidens nicht zwischen den Fall-Clustern für die unterschiedliche Kategorisierungsurteile erwartet wurden, während die Versuchspersonen in der Bedingung bewussten Denkens dies taten. Im folgenden Abschnitt werden diese Resultate näher erörtert.

Ausgehend von den in Experiment 1 gemachten Erfahrungen wurden die Fälle in Experiment 2 so gewählt, dass Unterschiede in den Kategorisierungsurteilen nicht auf Tallying als Entscheidungsstrategie zurückgeführt werden können. Stattdessen mussten kausale Faktoren (der kausale Status der Variablen bzw. die kausale Kohärenz der präsentierten Fälle) berücksichtigt werden, um zu differentiellen Kategorisierungsurteilen zu gelangen. Die Ergebnisse der Kategorisierungsaufgaben in Experiment 2 zeigen, dass lediglich die Versuchspersonen in der Bedingung bewussten Nachdenkens mit selbstgewählter Dauer in ihren Ratings signifikante Unterschiede zwischen Fällen mit Variablen mit hohem und niedrigem kausalen Status bzw. hoher und niedriger kausaler Kohärenz machen. Zusammen mit dem marginal signifikanten Unterschied in der Bedingung unbewussten Denkens kann dies als Indiz dafür aufgefasst werden, dass es bei den in Experiment 2 verwendeten Kategorisierungsaufgaben tendenziell vorteilhaft ist, nach der Stimuluspräsentation noch etwas Zeit bis zur Entscheidung zur Verfügung zu haben unabhängig davon, ob diese für bewusstes oder unbewusstes Nachdenken genutzt wird. Dieser Vorteil ist jedoch nicht so groß, dass sich statistisch eine Überlegenheit gegenüber dem sofortigen Entscheiden nach der Stimuluspräsentation nachweisen ließe. Somit sind die Ergebnisse im Hinblick auf die aufgestellte Hypothese uneinheitlich: Auf der einen Seite konnte zwar keine Überlegenheit bewussten Denkens gegenüber spontanen Entscheidungen nachgewiesen werden. Auf der anderen Seite waren die Versuchspersonen in der Bedingung spontanen Entscheidens jedoch nicht in der Lage überhaupt zwischen den Fall-Clustern zu 
unterscheiden, während dies bei den Versuchspersonen in der Bedingung bewussten Nachdenkens mit selbstgewählter Dauer der Fall war.

In Bezug auf die aufgestellte Hypothese 2 sind die Ergebnisse der Interventionsaufgaben in Experiment 2 ebenfalls nur schwer zu bewerten. Das NichtVorhandensein einer Überlegenheit von Urteilen, die nach einer Phase bewussten Denkens getroffen wurden gegenüber solchen, die unmittelbar nach der Stimuluspräsentation abgegeben wurden spricht eindeutig gegen die Gültigkeit der Hypothese. Auf der anderen Seite gelang es den Versuchspersonen jedoch in keiner der Bedingungen überhaupt zwischen den Fall-Clustern, die eine Präferenz für eine bestimmte Intervention besaßen, zu differenzieren. Die relativ eindeutigen Ergebnisse von Experiment 1 konnten zeigen, dass Versuchspersonen generell in der Lage sind Interventionsentscheidungen auf der Grundlage von Wissen über ein komplexes Kausalsystem zu treffen. Daher bleibt die Frage nach den Gründen für die mangelnde Differenzierung zwischen den Fall-Clustern in Experiment 2. Ein möglicher Grund für die schlechten Differenzierungsleistungen könnte sein, dass der Unterschied in der Anzahl von Variablen, die durch die beiden möglichen Interventionen beeinflusst werden konnten in allen vier Fällen lediglich 1 betrug. Im Gegensatz dazu betrug der Unterschied zwischen den beiden möglichen Interventionen bei den in Experiment 1 präsentierten Fällen jeweils 2 bzw. 3 Variablen. Der geringere Unterschied in Experiment 2 könnte dazu geführt haben, dass die Versuchspersonen beide möglichen Interventionen als ähnlich effektiv angesehen und dementsprechend beurteilt haben. Insgesamt konnte die aufgestellte Hypothese zu den Bedingungen mit Interventionsaufgaben somit nicht bestätigt werden.

\subsection{Experiment 3}

\subsubsection{Ziel des Experiments}

Eine Konsequenz aus den im Hinblick auf die aufgestellten Hypothesen uneinheitlichen Ergebnissen der ersten beiden Experimente bestand darin, Kategorisierungsund Interventionsaufgaben in weiteren Experimenten zu untersuchen. Im nun vorzustellenden Experiment 3 wurden die kognitiven Verarbeitungsprozesse bei kausalen Kategorisierungsaufgaben untersucht, während Experiment 4 der Untersuchung von Verarbeitungsprozessen bei Interventionsaufgaben dient. Darüber hinaus wurden mit der Durchführung von Experiment 3 folgende Ziele verfolgt. Zum einen sollte weitere empirische Evidenz zum Einfluss verschiedener Verarbeitungsbedingungen auf 
Kategorisierungsentscheidungen erbracht werden. Dabei sollte überprüft werden, ob sich die Befunde aus Experiment 2 mit anderem Material replizieren lassen oder ob es doch möglich sein kann, unmittelbar im Anschluss an die Stimuluspräsentation $\mathrm{zu}$ korrekten Kategorisierungsurteilen auf der Basis eines zuvor erlernten Kausalsystems zu gelangen. Daneben sollte geprüft werden, wie viel Zeit sich Versuchspersonen für das Betrachten der Stimuli nehmen, um bereits während der Stimuluspräsentation zu einem Urteil gelangen zu können. Zu diesem Zweck wurde die neue Bedingung der Online-Verarbeitung in Experiment 3 aufgenommen. Diese Bedingung gleicht der Bedingung spontanen Entscheidens bis auf eine Ausnahme: Während die Dauer der Präsentation einer einzelnen Variablenausprägung in den Bedingungen spontanen Entscheidens auf ca. 1,5 Sekunden festgelegt ist, können die Versuchspersonen in den Bedingungen der Online-Verarbeitung selbst bestimmen, wie lange sie eine bestimmte Variablenausprägung betrachten wollen. Den Versuchspersonen war sowohl in der Bedingung spontanen Entscheidens als auch in der Bedingung der OnlineVerarbeitung bekannt, dass sie unmittelbar im Anschluss an die Stimuluspräsentation innerhalb kurzer Zeit ein Urteil abgeben sollten. Die zeitliche Restriktion in der Bedingung spontanen Entscheidens sollte bewusstes Nachdenken über die Aufgabe verhindern. Für die Bedingung der Online-Verarbeitung wird hingegen davon ausgegangen, dass die Versuchspersonen die ihnen während der Präsentation zusätzlich zur Verfügung stehende Zeit bewusst für die Urteilsfindung eingesetzt haben. Die Bedingung der Online-Verarbeitung soll somit Typ 2-Informationsverarbeitungsprozesse bereits während der Stimuluspräsentation ermöglichen. Es wird daher angenommen, dass die Bedingung der Online-Verarbeitung zu ähnlichen Resultaten führen wird wie die Bedingung des bewussten Nachdenkens mit selbstgewählter Dauer. In beiden Bedingungen ist es den Versuchspersonen möglich, für eine von ihnen selbst bestimmbare Dauer über die Lösung der Aufgabe mittels Typ 2-Prozessen nachzudenken. Dies geschieht bei der Bedingung der Online-Verarbeitung während der Stimuluspräsentation und in der Bedingung des bewussten Nachdenkens unmittelbar im Anschluss an diese. Der ursprünglichen Hypothese folgend wird des Weiteren vorhergesagt, dass diese Typ 2-Verarbeitungsprozesse sowohl in der Bedingung der Online-Verarbeitung als auch in der Bedingung des bewussten Denkens mit selbstgewählter Dauer nicht zu signifikant besseren Kategorisierungsleistungen führen als die Bedingung spontanen Entscheidens, in der auf Typ 1-Verarbeitungsprozesse basierende Urteile abgefragt wurden. 


\subsubsection{Stichprobe und experimentelles Design}

Insgesamt nahmen 106 Versuchspersonen an Experiment 3 teil. Experiment 3 kann als 4 (Verarbeitungsbedingung: Spontan vs. Unbewusst vs. Bewusst mit selbstgewählter Dauer vs. Online-Verarbeitung) x 5(präsentierte Fälle) Design charakterisiert werden. Die Variation der Verarbeitungsbedingungen erfolgte zwischen den Versuchspersonen, die der präsentierten Fälle innerhalb der Versuchspersonen.

\subsubsection{Ablauf und verwendetes Stimulusmaterial}

Die wesentliche Veränderung in Experiment 3 bestand im Hinzufügen der weiter oben bereits beschriebenen Bedingung der Online-Verarbeitung. Diese Bedingung sollte es den Versuchspersonen erlauben, sich bestmöglich auf das unmittelbar nach der Präsentation der Variablenausprägungen eines Falles abzugebende Urteil bezüglich dessen Kategorienzugehörigkeit vorzubereiten. Die Versuchspersonen in der Bedingung der OnlineVerarbeitung wurden über eine modifizierte erste Übungsaufgabe (vgl. Abschnitt 4.1) mit der neuen Bedingung vertraut gemacht, in der sie selbst (mittels Mausklick auf eine eingeblendete und mit „Weiter“ beschriftete Schaltfläche) entscheiden konnten, wie lange sie ein zu einer zuvor präsentierten Frage gehörendes und im Anschluss zu beurteilendes Bild betrachten wollten. In der späteren Testphase des Experimentes konnten die Versuchspersonen mit Klick auf die „Weiter"-Schaltfläche bestimmen, wie lange sie die verschiedenen Variablenausprägungen betrachten wollten. Neben der Bedingung der Online-Verarbeitung wurden außerdem die Bedingungen des spontanen Entscheidens, des unbewussten Nachdenkens, sowie des bewussten Nachdenkens mit selbstgewählter Dauer in Experiment 3 durchgeführt. Der Ablauf dieser weiteren Bedingungen entsprach der Darstellung in Experiment 2.

Nachdem die bedingungsspezifische Übungsphase von den Versuchspersonen durchlaufen worden war, wurde den Versuchspersonen das in eine Coverstory eingebettete Kausalsystem präsentiert. Die Aufgabe der Versuchspersonen bestand darin, verschiedene Patienten hinsichtlich des Vorliegens einer neuen psychischen Störung, der sog. PostEmbitterment-Disorder zu kategorisieren. Das in Experiment 3 verwendete Kausalsystem wurde als neu entwickeltes Störungsmodell beschrieben und bestand aus 13 Variablen, die mittels 12 Kausalrelationen miteinander verbunden sind (s. Abbildung 18). Die Variablen stellten Symptome der Post-Embitterment-Disorder dar, die bei einem Patienten entweder anwesend oder abwesend sein können. Durch die Konstellation der Symtome eines Patienten 
sollte es möglich sein, die Wahrscheinlichkeit des Vorliegens der Post-Embitterment-Disorder zu schätzen. Wie in Experiment 2 wurden keine expliziten Basisraten für die verschiedenen Variablen genannt. Allerdings wurde ausdrücklich darauf hingewiesen, dass nicht alle Symptome zwangsläufig vorliegen müssen, um eine Diagnose der Störung vornehmen zu können. Zur besseren Veranschaulichung werden im Folgenden die Instruktionen bezüglich des in Experiment 3 verwendeten Kausalsystems wiedergegeben. Die Darstellung des Kausalsystems wurde wie in Experiment 2 in zwei Stufen balanciert, um Effekte der Position der Systemvariablen kontrollieren zu können.

\subsubsection{Instruktionen Kausalsystem Post-Embitterment-Disorder}

„Stelle Dir bitte folgende Situation vor: In der jüngsten Vergangenheit wurden in der psychotherapeutischen Praxis vermehrt Fälle registriert, die eine psychische Störung nach einem Verbitterung auslösenden Ereignis aufweisen. Ein solches Ereignis kann z.B. der Verlust einer langjährigen Arbeitsstelle ohne ersichtlichen Grund oder ein Betrug durch nahe Freunde bzw. Angehörige sein. Auf Grundlage systematischer Erhebungen der Symptome von Patienten, die ein Verbitterung auslösendes Ereignis erlebt haben, wurde ein Krankheitsmodell entwickelt. Dieses beschreibt, wie die Symptome der sog. „PostEmbitterment Disorder" miteinander in Zusammenhang stehen. Es wurde festgestellt, dass sich bestimmte Symptome der „Post-Embitterment Disorder“ gegenseitig bedingen und dadurch häufig in bestimmten Konstellationen auftreten.

Wir wollen Dir nun erst einmal das Störungsmodell der „,Post-Embitterment Disorder “ vorstellen, das Du Dir bitte so gut wie möglich einprägen sollst: Es ist wichtig zu beachten, dass alle Symptome zwar oft auftreten, aber es müssen nicht alle Symptome zwangsläufig vorliegen, um eine Diagnose „Post-Embitterment Disorder“ stellen zu können. Für eine Diagnose ist immer die jeweilige Symptomkonstellation vor dem Hintergrund des Störungsmodells relevant.

Es wird angenommen, dass viele Patienten auf ein Verbitterung auslösendes Ereignis mit gefühlten Kontrollverlust reagieren. Gleichzeitig kommt es auch zu einer starken Stressreaktion. Das Gefühl des Kontrollverlustes und die starke Stressreaktion können auch lange nach Beendigung des Verbitterung auslösenden Ereignisses anhalten. Der wahrgenommene Kontrollverlust führt oftmals zu verminderten Selbstwirksamkeitserwartungen. Die verminderten Selbstwirksamkeitserwartungen führen oftmals zu Pessimismus. Außerdem führen die verminderten Selbstwirksamkeitserwartungen oftmals zu einer wahrgenommenen Handlungsunfähigkeit. Die wahrgenommene Handlungsunfähigkeit führt oftmals zu einer Reduzierung der Teilnahme an sozialen Aktivitäten. Die Reduzierung der Teilnahme an sozialen Aktivitäten führt oftmals zu einem Gefühl der Entfremdung von Anderen.

Eine durch ein Verbitterung auslösendes Ereignis ausgelöste starke Stressreaktion führt oftmals zu anhaltenden Symptomen erhöhter Erregung. Anhaltende Symptome erhöhter Erregung führen oftmals zu Reizbarkeit. Außerdem führen die anhaltenden Symptome erhöhter Erregung oftmals zu Schwierigkeiten einoder durchzuschlafen. Schwierigkeiten ein-oder durchzuschlafen führen oftmals zu anhaltender Müdigkeit am 
Tage. Anhaltende Müdigkeit am Tage führt oftmals zu Konzentrationsschwierigkeiten. Deine Aufgabe wird es sein, zu bestimmen wie wahrscheinlich bei verschiedenen Patienten eine „Post-Embitterment Disorder“ vorliegt."

Die verbale Beschreibung des Kausalsystems wurde anschließend mittels einer Graphik veranschaulicht (s. Abbildung 18). Die Balancierung der graphischen Darstellung des Kausalsystems bestand in einer Spiegelung der von der Variablen „Verbitterung auslösendes Ereignis“" ausgehenden Variablen: Diejenigen Variablen, die sich in Balancierungsbedingung 1 auf der linken Seite der Abbildung befanden, wurden in Balancierungsbedingung 2 auf der rechten Seite dargestellt. Die Balancierung sollte Effekte der Position der Variablen im Kausalsystem kontrollierbar machen.

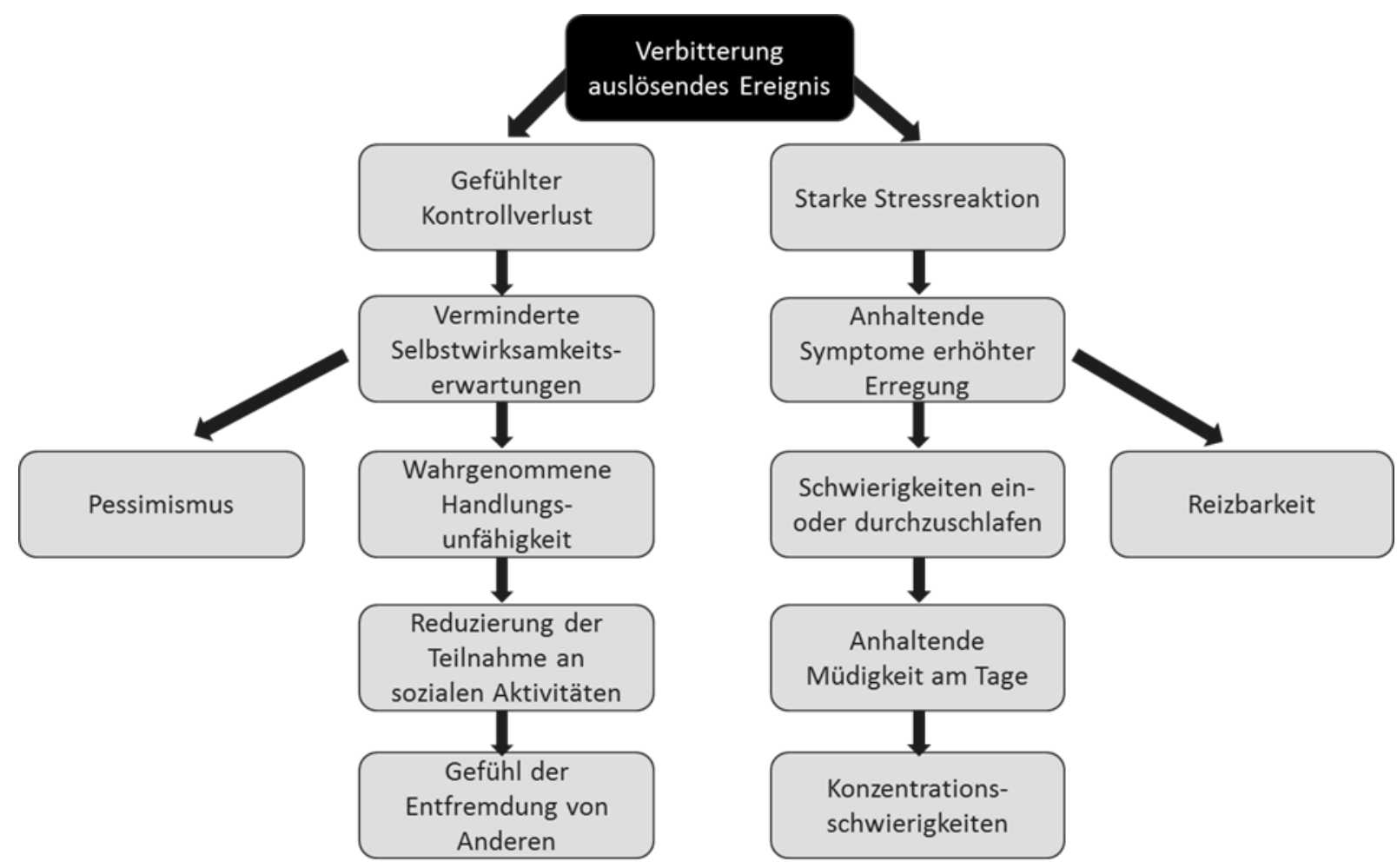

Abbildung 18: Darstellung des Kausalsystems in Experiment 3 (Balancierungsbedingung 1). Die Pfeile zwischen den Variablen repräsentieren Kausalrelationen und sind von der verursachenden Variablen auf den jeweiligen Effekt gerichtet. Die in dieser Abbildung dargestellten Variablenausprägungen stellen die verschiedenen Symptome der Post-Embitterment Disorder dar. Die Abwesenheit einzelner Symptome wird während der Stimuluspräsentation durch das Wort „kein“ bzw „keine“ vor dem Namen des Symptoms gekennzeichnet. Die Variable „Verbitterung auslösendes Ereignis“ wurde bei allen Fällen als vorhanden vorausgesetzt und nicht während der Stimuluspräsentation gezeigt.

\subsubsection{Multiple-Choice Wissenstest}

Der nach der Instruktion des Kausalsystems erfolgende Wissenstest bestand in Experiment 3 aus 10 Fragen mit je 4 Antwortalternativen (s. Anhang D). Konnte eine Versuchsperson nicht alle Fragen korrekt beantworten, wurden ihr die Instruktionen für das 
Kausalsystem und im Anschluss die Multiple-Choice-Fragen nochmals präsentiert. Nur Versuchspersonen, die alle Fragen korrekt beantworten konnten, auch nach sechs Durchgängen mindestens einen Fehler machten oder nach Ablauf von 30 Minuten die Lernphase des Experimentes nicht abgeschlossen hatten, kamen in die Testphase des Experiments. Bei den letzten beiden Personengruppen wurden die Datensätze jedoch als ungültig markiert, um sie von der später erfolgenden Analyse ausschließen zu können (vgl. Abschnitt 4.1).

\subsubsection{Testphase}

Die Versuchspersonen hatten in der Testphase von Experiment 3 insgesamt fünf Versuchsdurchgänge zu absolvieren, die jeweils in der Beurteilung eines Falles bestanden. Die Reihenfolge der zu beurteilenden Fälle wurde für jede Versuchsperson randomisiert. Die von den Versuchspersonen zu beantwortende Frage: „Wie wahrscheinlich ist es, dass bei Patient X eine Post-Embitterment Disorder vorliegt?“" wurde zunächst für ca. 3 Sekunden gezeigt, wobei X einen dem aktuellen Versuchsdurchgang entsprechenden Buchstaben (A, B, C, D oder E) darstellte. Unmittelbar im Anschluss wurden die Variablenausprägung eines Falles in randomisierter Reihenfolge in der Mitte des Bildschirms präsentiert. Die möglichen Ausprägungen einer Variablen kennzeichneten die An- oder Abwesenheit eines Symptoms beim aktuell zu beurteilenden Fall. War ein Symptom anwesend, so wurde dessen Name in einer den Versuchspersonen aus der Lernphase des Experiments bekannten optischen Form dargeboten. War ein Symptom abwesend, so wurde vor dessen Name das Wort „kein“ bzw. „keine“ eingefügt (z.B. „kein gefühlter Kontrollverlust“; „keine starke Stressreaktion“). Da die Versuchspersonen instruiert wurden, dass alle zu beurteilenden Patienten ein Verbitterung auslösendes Ereignis erlebt hatten, wurde auf die Präsentation dieser Variable verzichtet. Somit bestand ein Fall aus insgesamt 12 Variablenausprägungen. Die Dauer der Darstellung der einzelnen Variablenausprägungen betrug 1,5 Sekunden für die Bedingungen des spontanen Entscheidens, des unbewussten Denkens, sowie des bewussten Denkens mit selbstgewählter Dauer. In der Bedingung der Online-Verarbeitung konnten die Versuchspersonen selbst entscheiden, wie lange sie die aktuell präsentierte Variablenausprägung betrachten wollten. In den Bedingungen des spontanen Entscheidens und der Online-Verarbeitung erfolgte unmittelbar nach der Präsentation der letzten Variablenausprägung eines Falles die abermalige Präsentation der zu beantwortenden Frage für ca. 3 Sekunden. Danach wurde zusätzlich zur Frage die Antwortskala, die aus elf mit in 1er Schritten ansteigenden Zahlenwerten von -5 bis +5 beschrifteten Schaltflächen bestand 
eingeblendet, auf denen die Versuchspersonen mittels Mausklick ihre Einschätzung abgeben konnten. Der linke Pol der Skala (-5) war mit „,sehr unwahrscheinlich“, der rechte Pol (+5) mit „sehr wahrscheinlich“ gekennzeichnet. In den Bedingungen des unbewussten Denkens und des bewussten Nachdenkens mit selbstgewählter Dauer hatten die Versuchspersonen im Anschluss an die Stimuluspräsentation zunächst die der jeweiligen Verarbeitungsbedingung entsprechende Aufgabe zu absolvieren. Antwortete eine Versuchsperson nicht innerhalb des vorgesehenen 3-sekündigen Zeitfensters wurde unmittelbar nach Abgabe der verspäteten Einschätzung ein Hinweis mit der Aufforderung eingeblendet, beim nächsten Durchgang schneller zu antworten.
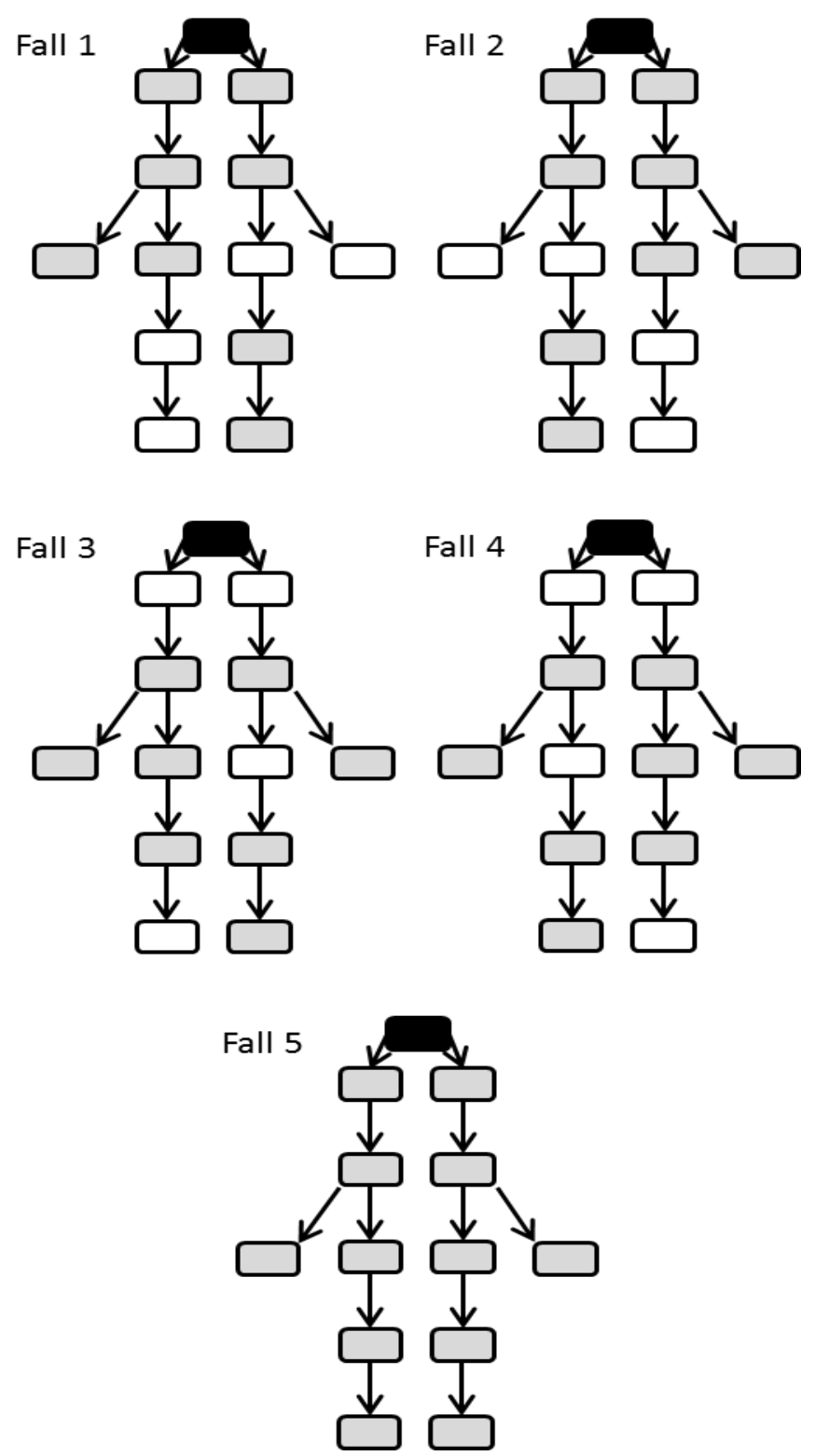

Abbildung 19:Schematische Darstellung der In Experiment 3 präsentierten Fälle (Balancierungsbedingung 1. Die Felder repräsentieren die Variablen des Kausalsystems, die Pfeile die kausalen Verbindungen zwischen den Variablen, wobei die Pfeilspitze auf den Effekt in der jeweiligen Relation weist. Grau gefärbte Felder repräsentieren die anwesenden Symptome, weiß gefärbte Felder die abwesenden Symptome. 
Abbildung 19 stellt die fünf in Experiment 3 verwendeten Fälle graphisch dar, zu denen folgende Vorhersagen gemacht wurden: Für Fall 5, bei dem alle möglichen Symptome der Post-Embitterment Disorder anwesend waren wurden die höchsten Ratings in Bezug auf die Einschätzung der Wahrscheinlichkeit des Vorliegens der Störung erwartet. Dieser Fall diente daher als eine Art von Kontrolle. Bei den Fällen 1-4 waren jeweils 8 von 12 möglichen Symptomen anwesend. Allerdings kann sowohl auf Grundlage des kausalen Status der anwesenden Variablen (Hypothese 1) als auch auf Basis der kausalen Kohärenz die Vorhersage gemacht werden, dass die Fälle 1 und 2 für höhere Einschätzungen der Wahrscheinlichkeit des Vorliegens der Post-Embitterment Disorder führen sollten als die Fälle 3 und 4.

\subsubsection{Ergebnisse}

16 der 106 Versuchspersonen in Experiment 3 konnten aufgrund von technischen Problemen (z.B. Abstürzen des Computerprogramms) das Experiment nicht bis zum Ende durchführen. Von den verbleibenden 90 Versuchspersonen bestanden 85 den Multiple-Choice Wissenstests, mit dem überprüft wurde, dass das instruierte Kausalsystem auch tatsächlich gelernt wurde. Zwei Versuchspersonen brachen das Experiment vorzeitig ab, so dass die Daten von 83 Versuchspersonen in die folgenden Analysen eingehen. Von diesen 83 Versuchspersonen befanden sich 20 in der Bedingung spontanen Entscheidens, 19 in der Bedingung unbewussten Denkens, 20 in der Bedingung bewussten Nachdenkens mit selbstgewählter Dauer und 24 in der Bedingung der Online-Verarbeitung. Der Anteil der weiblichen Probanden betrug 58\%. Die Versuchspersonen waren allesamt Studierende der Universität Göttingen. Die größte Gruppe bildeten Studierende der Psychologie mit einem Anteil von 40\%. Im Durchschnitt benötigten die Versuchspersonen 23 Minuten für die Durchführung von Experiment 3 (spontanes Entscheiden: 17 Minuten; unbewusstes Denken: 30 Minuten; bewusstes Denken mit selbstgewählter Dauer: 22 Minuten; Online-Verarbeitung: 22 Minuten). Die durchschnittliche Zeitspanne, die die Versuchspersonen in der Bedingung bewussten Denkens mit selbstgewählter Dauer zum Überlegen benötigten betrug 16,5 Sekunden. Keine der Versuchspersonen in dieser Bedingung benötigte die maximal zur Verfügung stehende Nachdenk-Zeit von 2 Minuten.

Für die im folgenden berichteten Analysen der in Experiment 3 abgegebenen Urteile wurden zunächst die Ratings für Fälle für die gleiche Kategorisierungsurteile erwartet wurden (hohe erwartete Kategorisierungsurteile für die Fälle 1 und 2, sowie niedrige erwartete 
Kategorisierungsurteile für die Fälle 3 und 4, vgl. Abschnitt 4.4.3.3) auf Ebene der Versuchspersonen gemittelt. Zusammen mit den Urteilen für Fall 5, bei dem die höchsten Ratings erwartet wurden, basiert die Analyse der Daten in Experiment 3 somit auf 3 FallClustern.

\subsubsection{Multiple-Choice Wissenstest}

Lediglich 5 von 90 (6\%) der Versuchspersonen konnte auch nach sechs Durchgängen nicht alle Fragen des für alle Bedingungen identischen Wissenstests korrekt beantworten. Die verbleibenden 85 Versuchspersonen benötigten über alle Bedingungen im Durchschnitt 2,18 Durchgänge um alle Fragen korrekt zu beantworten (vgl. Abbildung 20). Die Verarbeitungsbedingung übte keinen signifikanten Einfluss auf die Anzahl der Lerndurchgänge aus $(F(2,81)<1, p=0,84, M S E<1)$.

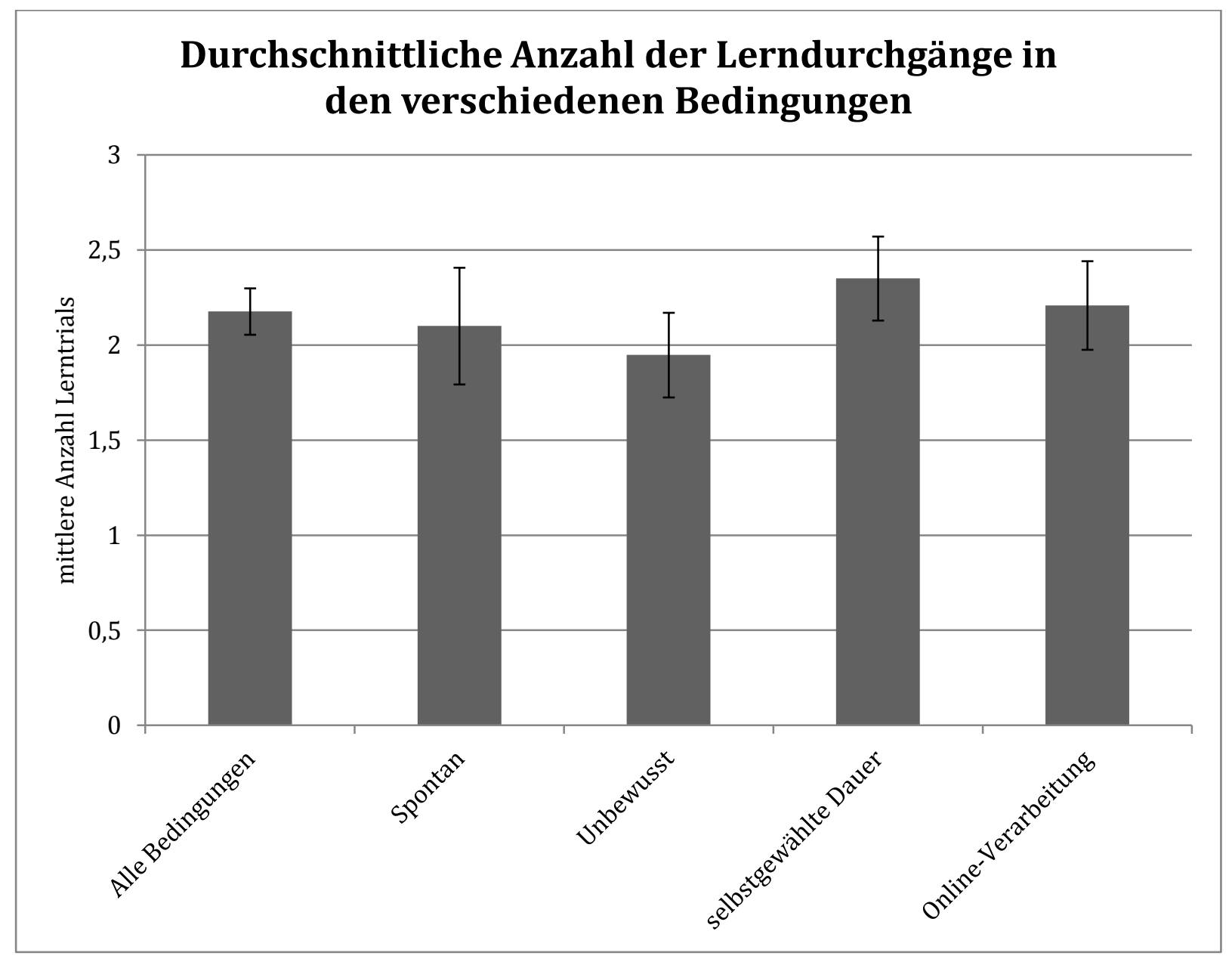

Abbildung 20: Durchschnittliche Anzahl der von den Versuchspersonen in den verschiedenen Bedingungen von Experiment 3 benötigten Durchgänge (und deren Standardfehler) beim Multiple-Choice Wissenstest. 


\subsubsection{Dauer der Online-Verarbeitung}

Eine Besonderheit von Experiment 3 war die Existenz der Bedingung der OnlineVerarbeitung, die es den Versuchspersonen ermöglichte, die verschiedenen Variablenausprägungen so lange zu betrachten wie sie wollten. Diese Bedingung sollte es somit ermöglichen, ohne Zeitdruck bereits während der Stimuluspräsentation zu einem Urteil $\mathrm{zu}$ gelangen und dieses direkt im Anschluss abzugeben. Aufgrund eines Programmierfehlers wurde die Betrachtungszeit der ersten präsentierten Variablenausprägung eines jeden Falles nicht aufgezeichnet. Daher liegen pro Fall und Versuchsperson lediglich Betrachtungszeiten für 11 der 12 präsentierten Variablenausprägungen vor. Durch die randomisierte Reihenfolge, in der die Variablenausprägungen präsentiert wurden, waren alle Variablen in ähnlicher Weise durch den Fehler betroffen. Da die Betrachtungszeiten zu Beginn der Sequenz von Variablenausprägungen eines Falles tendenziell länger waren als gegen Ende der Sequenz (vgl. Abbildung 21) sollte der Durchschnitt der 11 aufgezeichneten Betrachtungszeiten nicht höher sein, als der nicht ermittelbare Durchschnitt der Betrachtungszeiten für alle 12 Variablenausprägungen. Daher erscheint es gerechtfertigt, dass die mittlere aufgezeichnete Betrachtungszeit mit der in den übrigen Verarbeitungsbedingungen festgesetzten Betrachtungszeit von 1,5 Sekunden verglichen wird, um festzustellen, ob sich die Versuchspersonen in der Bedingung der Online-Verarbeitung mehr Zeit für die Betrachtung der Variablenausprägungen nahmen als ihnen in den anderen Bedingungen zur Verfügung stand. Die über alle Versuchspersonen und Fälle gemittelte Betrachtungszeit betrug 2,86 Sekunden. Die durchschnittlichen Betrachtungszeiten der einzelnen Versuchspersonen in der Bedingung der Online-Verarbeitung wurden mit einem 1-Stichproben t-Test gegen die auf 1,5 Sekunden festgesetzte Betrachtungszeit in den anderen Verarbeitungsbedingungen getestet. Es ergab sich eine signifikant längere Betrachtungsdauer der einzelnen Variablenausprägungen in der Bedingung der Online-Verarbeitung $\left(t_{(23)}=6,94, p<0,001\right)$. Dies gilt auch, wenn die Betrachtungsdauern bei den einzelnen Messzeitpunkten separat analysiert werden (alle $p$ 's $<0,001$ ). 


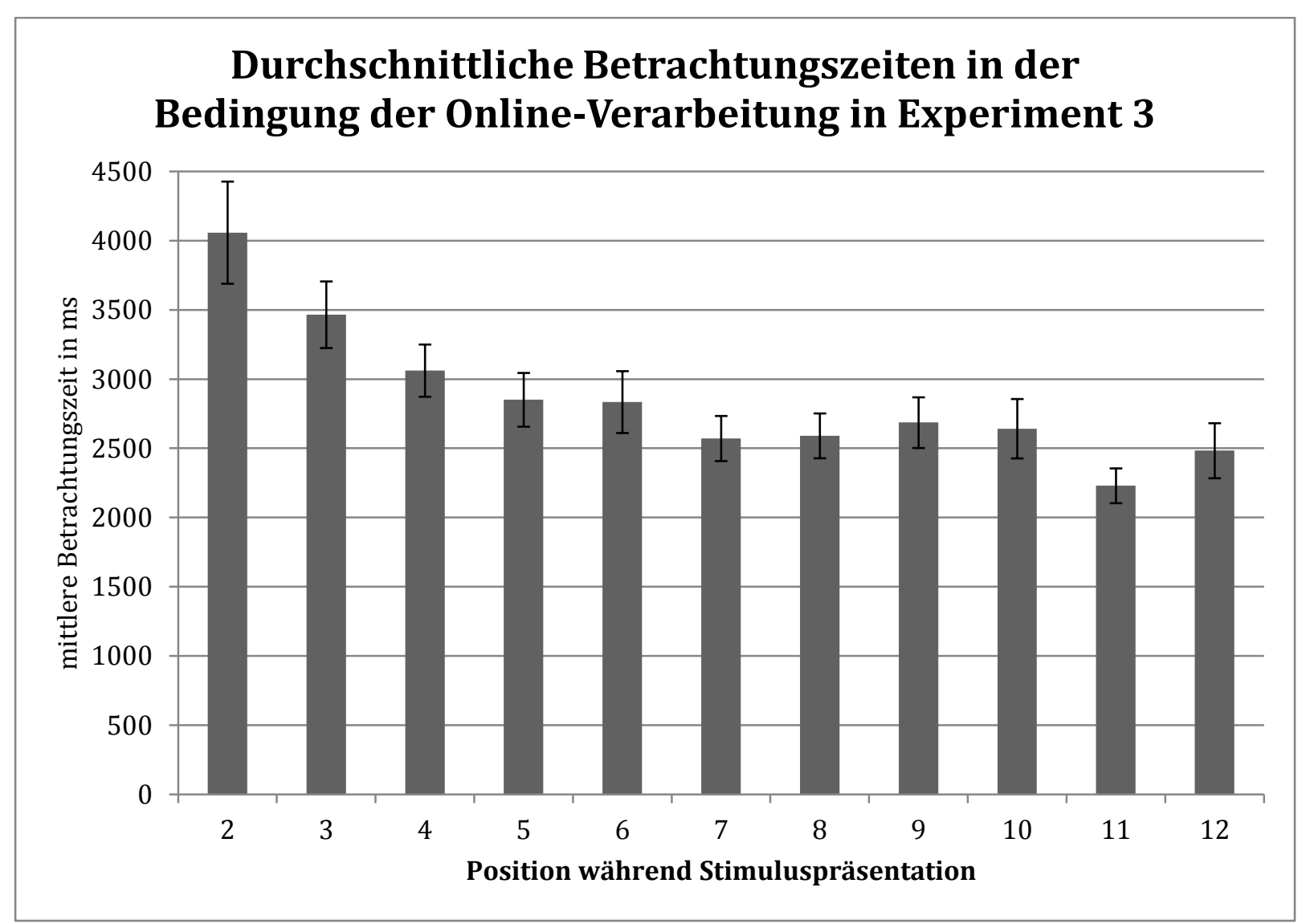

Abbildung 21: Durchschnittliche Betrachtungszeiten der Variablenausprägungen (gemittelt über alle Fälle) in Abhängigkeit ihrer Position während der Stimuluspräsentation (und zugehörige Standardfehler). Die Betrachtungszeiten der jeweils zu Beginn einer Sequenz präsentierten Variablenausprägungen wurden aufgrund eines Programmierfehlers nicht aufgezeichnet.

\subsubsection{Kategorisierungsurteile}

Die Prüfung der Gleichartigkeit der Antwortmuster für Urteile, die innerhalb von 3 bzw. 5 Sekunden nach Erscheinen der Antwortskala abgegeben wurden erfolgte mittels zweifaktorieller Varianzanalysen mit Messwiederholungen und ergab keine bedeutenden Unterschiede. Es wurde jeweils ein Haupteffekt für die nach erwarteten Ratings gebildeten Fall-Cluster $\quad(3$ Sekunden: $F(2,152)=185,67, \quad p<0,001, \quad M S E=41659 ; \quad 5$ Sekunden: $F(2,154)=193,43, p<0,001, M S E=41817)$ gefunden, während sowohl die Variation der Verarbeitungsbedingungen (3 Sekunden: $F(3,76)<1, p=0,911, M S E=60 ; 5$ Sekunden: $F(3,77)<1, p=0,85, M S E=89)$ als auch die Interaktion zwischen Verarbeitungsbedingungen und Fall-Clustern (3 Sekunden: $F(6,152)<1, p=0,54, M S E=189$; 5 Sekunden: $F(6,154)=1,03$, $p=0,41, M S E=223)$ keine signifikanten Effekte nach sich zog. Auch das Ersetzen der fehlenden Werte durch den über alle Bedingungen gebildeten Durchschnitt für das jeweilige Fall-Cluster resultierte nicht in Veränderungen des Antwortmusters (Fall-Cluster: $F(2,158)=198,40, p<0,001, M S E=42988 ;$ Verarbeitungsbedingungen: $F(3,79)<1, p=0,90$, $M S E=63$; Interaktion zwischen Verarbeitungsbedingungen und Fall-Clustern: $F(6,158)=1,54$, 
$p=0,17, M S E=333)$. Daher werden für die im Folgenden vorgestellten Kontrastanalysen die Daten verwendet, die auf innerhalb von 5 Sekunden abgegebenen Urteilen basieren und bei denen die fehlenden Werte ersetzt wurden. Eine graphische Darstellung der durchschnittlichen Ratings auf Grundlage dieser Daten liefert Abbildung 22.

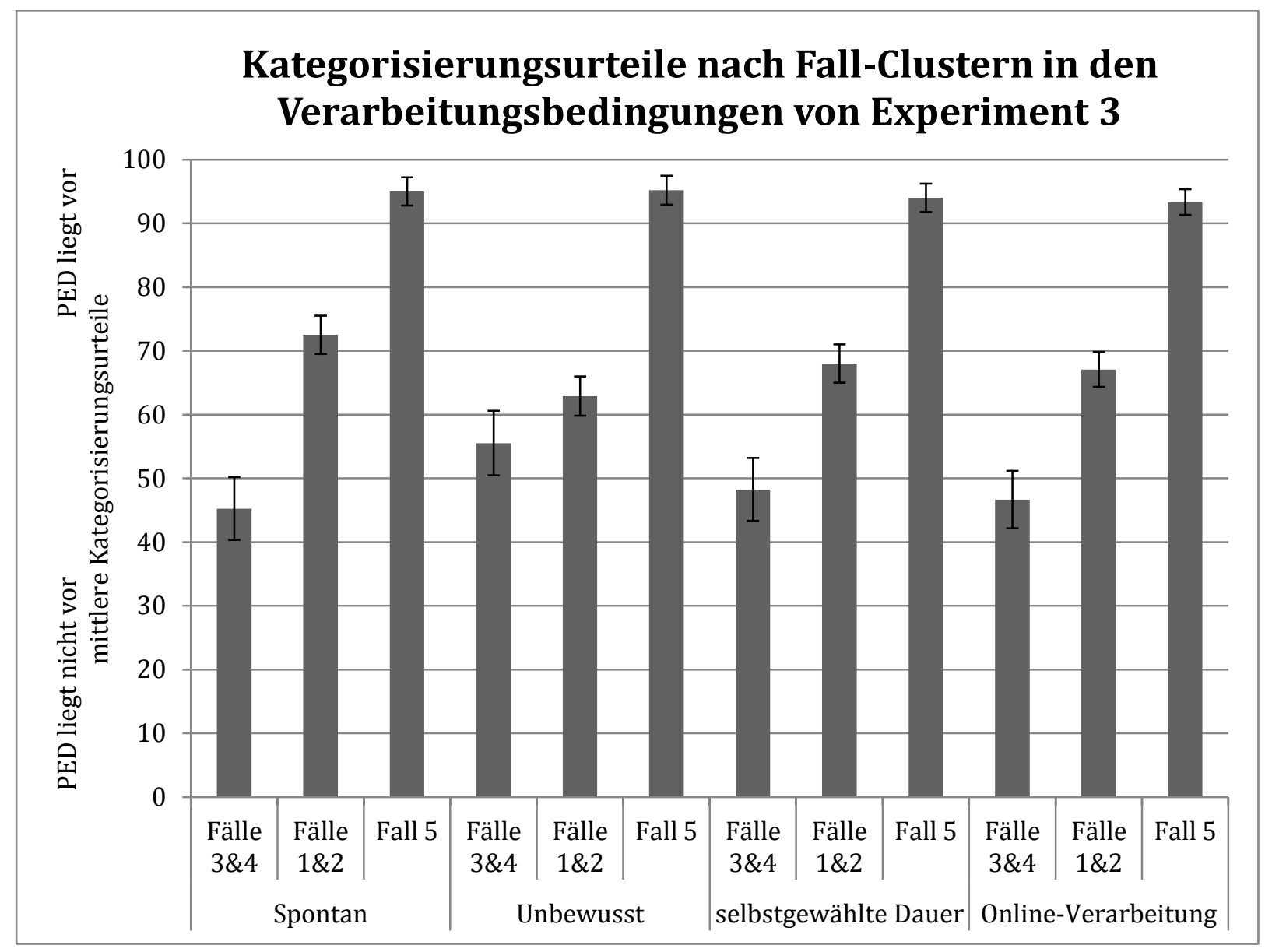

Abbildung 22: Durchschnittliche Kategorisierungsurteile (und Standardfehler) für die auf unterschiedlichen erwarteten Ratings basierenden Fall-Cluster in den verschiedenen Verarbeitungsbedingungen von Experiment 3. Die Anordnung der Fall-Cluster innerhalb der Verarbeitungsbedingungen erfolgte nach ansteigendem erwartetem Rating.

Wie aus Tabelle 6 ersichtlich, differenzierten die Versuchspersonen in Experiment 3 über alle Bedingungen betrachtet zwischen den verschiedenen Fall-Clustern. Eine Analyse der einzelnen Verarbeitungsbedingungen ergab, dass dieser Effekt auf den Urteilen von Versuchspersonen in den Bedingungen des sofortigen Entscheidens, des bewussten Nachdenkens mit selbstgewählter Dauer, sowie der Bedingung der Online-Verarbeitung basierte. Die Versuchspersonen in der Bedingung des unbewussten Denkens bedachten Fall 5 (bei dem alle für das Vorliegen der Post-Embitterment-Disorder charakteristischen Variablenausprägungen vorlagen) zwar mit den höchsten Kategorisierungsurteilen. Sie differenzierten allerdings nicht zwischen den Fall-Clustern, die sich durch die kausalen Faktoren des kausalen Status der vorliegenden für die PED charakteristischen 
Variablenausprägungen (nicht aber durch deren Anzahl) bzw. der kausalen Kohärenz voneinander unterschieden und dadurch den kritischen Test für die Differenzierungsleistung darstellten.

Tabelle 6: Kontraste zwischen Fall-Clustern mit unterschiedlichen erwarteten Kategorisierungsurteilen in den verschiedenen Verarbeitungsbedingungen von Experiment 3.

\begin{tabular}{|c|c|c|c|c|c|}
\hline Kontrast & Alle & Spontan & Unbewusst & $\begin{array}{c}\text { Selbstgewählte } \\
\text { Dauer }\end{array}$ & $\begin{array}{c}\text { Online- } \\
\text { Verarbeitung }\end{array}$ \\
\hline $\begin{array}{l}\text { (I) Fälle } 1 \& 2 \text { vs. } \\
\text { Fälle } 3 \& 4\end{array}$ & $\begin{array}{c}t_{(82)}=7,44, \\
p<0,001\end{array}$ & $\begin{array}{l}t_{(19)}=5,34, \\
p<0,001\end{array}$ & $\begin{array}{c}t_{(18)}=1,41, \\
p=0,16\end{array}$ & $\begin{array}{c}t_{(19)}=3,87, \\
p<0,001\end{array}$ & $\begin{array}{c}t_{(23)}=4,38, \\
p<0,001\end{array}$ \\
\hline $\begin{array}{l}\text { (II) Fall } 5 \text { vs. } \\
\text { Fälle } 1 \& 2\end{array}$ & $\begin{array}{c}t_{(82)}=17,52, \\
p<0,001\end{array}$ & $\begin{array}{c}t_{(19)}=9,45, \\
p<0,001\end{array}$ & $\begin{array}{c}t_{(18)}=7,34, \\
p<0,001\end{array}$ & $\begin{array}{c}t_{(19)}=8,69, \\
p<0,001\end{array}$ & $\begin{array}{c}t_{(23)}=9,71, \\
p<0,001\end{array}$ \\
\hline
\end{tabular}

Eine weitere Analyse für den kritischen Kontrast (I) in Tabelle 6 zwischen den verschiedenen Verarbeitungsbedingungen ergab, dass die Versuchspersonen in der Bedingung des spontanen Entscheidens eine signifikant bessere Differenzierungsleistung zeigten als die Versuchspersonen in der Bedingung unbewussten Denkens $\left(t_{(37)}=2,72, p<0,01\right)$. Marginal signifikante Ergebnisse im Vergleich zur Bedingung unbewussten Denkens ergaben sich für die Bedingung bewussten Nachdenkens mit selbstgewählter Dauer $\left(t_{(37)}=1,69, p=0,09\right)$, sowie für die Bedingung der Online-Verarbeitung $\left(t_{(41)}=1,86, p=0,07\right)$. Entsprechend der Hypothese, dass bewusstes Nachdenken bei Kategorisierungsaufgaben keine Vorteile gegenüber dem sofortigen Entscheiden hat, konnte keine Überlegenheit der Bedingung des bewussten Entscheidens mit selbstgewählter Dauer $\left(t_{(38)}=-1,04, p=0,30\right)$ oder der Bedingung der OnlineVerarbeitung $\left(t_{(42)}=-0,99, p=0,33\right)$ gegenüber der Bedingung des spontanen Entscheidens ausgemacht werden. Die Differenzierungsleistung in der Bedingung der Online-Verarbeitung war nicht besser als in der Bedingung bewussten Nachdenkens mit selbstgewählter Dauer $\left(t_{(42)}=0,10, \quad p=0,92\right)$. Insgesamt zeigt sich somit, dass Versuchspersonen bei Kategorisierungsurteilen innerhalb kurzer Zeit kausale Faktoren berücksichtigen.

\subsubsection{Diskussion}

Die Ergebnisse von Experiment 3 bestätigen in vollem Umfang die Vorhersagen von Hypothese 1, dass Kategorisierungsurteile auf der Basis kausalen Wissens auch ohne das Wirken von Typ 2-Verarbeitungsprozessen während oder nach der Stimuluspräsentation vorgenommen werden können. Die Versuchspersonen in den Bedingungen des spontanen Entscheidens, des bewussten Nachdenkens mit selbstgewählter Dauer, sowie der Online- 
Verarbeitung waren allesamt in der Lage zwischen den Fall-Clustern zu differenzieren, die sich sowohl hinsichtlich des kausalen Status der vorhandene Variablen als auch hinsichtlich der kausalen Kohärenz voneinander unterschieden. Aufgrund der gleichen Anzahl an für die Diagnose der Post-Embitterment-Disorder charakteristischen Variablenausprägungen in vier der fünf präsentierten Fälle mussten die Versuchspersonen kausale Faktoren berücksichtigen, um zu unterschiedlichen Urteilen zu gelangen. Des Weiteren konnte gezeigt werden, dass weder die Bedingung der Online-Verarbeitung, in der die Versuchspersonen die einzelnen Variablenausprägungen signifikant länger betrachteten als in den übrigen Bedingungen, noch die Bedingung des bewussten Nachdenkens mit selbstgewählter Dauer zu besseren Urteilen führte als die Bedingung spontanen Entscheidens. Dagegen konnte eine signifikant bessere Differenzierung in der Bedingung spontanen Entscheidens gegenüber der Bedingung des unbewussten Denkens festgestellt werden. Auch die Versuchspersonen in den Bedingungen der Online Verarbeitung sowie des bewussten Denkens mit selbstgewählter Dauer erbrachten marginal signifikant bessere Differenzierungsleistungen als Versuchspersonen in der Bedingung des unbewussten Denkens. Da der entscheidende Unterschied zwischen der Bedingung des unbewussten Denkens und den übrigen Verarbeitungsbedingungen in der Auslastung des Arbeitsgedächtnisses mit für die Aufgabe irrelevanten Inhalten besteht ist es wahrscheinlich, dass eine Interferenz des während der Stimuluspräsentation gebildeten Urteils vorlag. Diese führte dazu, dass ein zuvor gebildetes Urteil während der Abfrage nicht mehr korrekt wiedergegeben wurde. Lediglich bei sehr eindeutigen Urteilen wie bei Fall 5 (hier waren alle Symptome der Post-Embitterment-Disorder anwesend) war es den Versuchspersonen möglich, ihr Urteil auch nach der Bearbeitung der Distraktoraufgabe korrekt wiederzugeben.

\subsection{Experiment 4}

\subsubsection{Ziel des Experiments}

Nachdem in Experiment 3 Belege für die Existenz schnell ablaufender Verarbeitungsprozesse bei Kategorisierungsaufgaben auf Grundlage eines komplexen Kausalsystems gesammelt werden konnten, richtet Experiment 4 seinen Fokus auf Interventionsaufgaben. Es knüpft damit an die Ergebnisse von Experiment 1 an, in dem gezeigt werden konnte, dass Typ 2-Verarbeitungsprozesse bei Interventionsaufgaben im Vergleich zu spontanen, auf Typ 1-Prozessen basierende Entscheidungen und Entscheidungen nach einer Phase unbewussten Denkens zu besseren Ergebnissen führen können. Ein Ziel von Experiment 4 war folglich, die Ergebnisse von Experiment $1 \mathrm{zu}$ replizieren. Darüber hinaus 
sollte die Stabilität des in Experiment 1 gefundenen Effektes untersucht werden. Dazu wurde ein neues Kausalsystem konstruiert, das mit einer neuen Coverstory kombiniert wurde. Darüber hinaus wurde den Versuchspersonen das Kausalsystem ausschließlich in Textform, also ohne erklärende Abbildungen, vermittelt. Auf Grundlage der aufgestellten Hypothesen sowie den Ergebnissen der Interventionsbedingungen von Experiment 1 wurde vorhergesagt, dass die Versuchspersonen in der Bedingung bewussten Nachdenkens, die eine Typ 2Informationsverarbeitung erlaubt, bei der Wahl der Interventionen für die präsentierten Fälle bessere Leistungen erbringen als die Versuchspersonen in der Bedingung spontanen Entscheidens, bei der lediglich Informationsverarbeitungsprozesse vom Typ 1 möglich sind.

\subsubsection{Stichprobe und experimentelles Design}

Insgesamt nahmen 86 Versuchspersonen an Experiment 4 teil. Experiment 4 kann als 1 (Aufgabe: Intervention) X 3 (Verarbeitungsbedingung: Spontan vs. Unbewusst vs. Bewusst mit festgelegter Dauer) X 7 (Fälle) Design charakterisiert werden, bei dem die Variation der Verarbeitungsbedingungen zwischen den Versuchspersonen und die Variation der zu beurteilenden Fälle innerhalb der Versuchspersonen erfolgte.

\subsubsection{Ablauf und verwendetes Stimulusmaterial}

Im Wesentlichen folgte der Ablauf von Experiment 4 der in Abschnitt 4.1 vorgestellten Prozedur. Da es sich um einen Replikationsversuch der in Experiment 1 getesteten Interventionsaufgaben handelte, wurden dieselben Verarbeitungsbedingungen gewählt und aus Gründen der Ökonomie auf die Erhebung einer zusätzlichen Bedingung des bewussten Nachdenkens mit selbstgewählter Dauer verzichtet. Somit wurden in Experiment 4 die Bedingungen sofortigen Entscheidens, des Entscheidens nach einer Phase unbewussten Denkens, sowie des Entscheidens nach einer Phase bewussten Denkens mit festgelegter Dauer erhoben. Die festgelegte Dauer der Bedingung bewussten Nachdenkens entsprach dabei der Dauer der Distraktoraufgabe in der Bedingung unbewussten Denkens (2 Minuten).

Nachdem die Versuchspersonen, die ihrer Bedingung entsprechende Übungsphase absolviert hatten, wurde ihnen in der anschließenden Lernphase das verwendete Kausalsystem mittels einer im Finanzbereich angesiedelten Coverstory präsentiert. Die Aufgabe, die die Versuchspersonen in Experiment $4 \mathrm{zu}$ absolvieren hatten, war die Entscheidung zwischen zwei (fiktiven) Schweizer Kantonalbanken, in denen sie in ihrer Rolle als Verkäufer/in einer Fondsgesellschaft verschiedene Aktienfonds vermarkten sollten. Dafür war es wichtig, etwas über die Vertriebsstruktur der Kantonalbanken in Erfahrung zu bringen, da diese in kausalen 
Beziehungen zueinander standen: Ein Fonds der von einer bestimmten Kantonalbank angeboten wird, wird mit einer 80\%-igen Wahrscheinlichkeit auch von denjenigen Kantonalbanken angeboten, deren Produktpolitik von der den Fonds anbietenden Bank beeinflusst wird. Die Stärke der im System vorhandenen Kausalbeziehungen ist somit 0,8. Darüber hinaus besteht auch die Möglichkeit, dass eine Kantonalbank unabhängig vom Angebot der sie beeinflussenden Bank einen Fonds mit einer 25\%-igen Wahrscheinlichkeit den Fonds selbständig in ihr Sortiment aufnimmt (d.h. die Basisrate liegt bei 0,25). Die Basisrate der Kantonalbank Zürich, die im System von keiner anderen Bank beeinflusst wird, liegt hingegen bei 0,6. Die Kantonalbank Zürich beeinflusst die Produktpolitik zweier Banken direkt und diese beiden Banken stellen die Punkte einer möglichen Intervention dar: Die Versuchsperson kann entscheiden, welche dieser beiden Banken einen Fonds mit 100\%-iger Wahrscheinlichkeit in ihr Sortiment aufnehmen soll. Dabei soll sie den Fonds in der Bank platzieren, die die Möglichkeit besitzt, die größere Anzahl anderer Kantonalbanken hinsichtlich der Aufnahme des Fonds in deren Sortiment zu beeinflussen. Die Beschreibung des in Experiment 4 verwendeten Kausalsystems war in 2 Stufen balanciert, um Vorwissenseffekte kontrollieren zu können. Zur besseren Veranschaulichung wird der Wortlaut der Instruktionen im Folgenden wiedergegeben.

\subsubsection{Instruktionen Kausalsystem Banken}

„,Wir kommen nun zum eigentlichen Experiment. Stelle Dir hierzu bitte folgende Situation vor: Du bist Verkäufer bei einer großen internationalen Fondsgesellschaft und u.a. für die Vermarktung neuer Aktienfonds bei Schweizer Kantonalbanken verantwortlich. Damit die neuen Fonds in möglichst vielen der Kantonalbanken den Kunden angeboten werden können (und Du eine möglichst hohe Provision bekommst), ist es wichtig etwas darüber zu wissen, welche der Banken die Produktpolitik der anderen Banken beeinflussen.

In Deinem Vertriebsgebiet gibt es insgesamt neun verschiedene Kantonalbanken (Zürich, Lausanne, Basel, Neuchâtel, Genève, Luzern, Winterthur, St. Gallen und Chur).

- Die Züricher Kantonalbank hat in diesem Bankenverbund normalerweise die Aufgabe, neue Aktienfonds zu bewerten und ggf. in ihr Angebot aufzunehmen. Über die letzten Jahre hat sich gezeigt, dass die Züricher Kantonalbank neue Fonds Deiner Fondsgesellschaft mit einer Wahrscheinlichkeit von 60 Prozent in ihr Sortiment aufnimmt.

- $\quad$ Die anderen Kantonalbanken suchen ebenfalls selbstständig nach neuen für sie relevanten Produkten. Jede dieser Banken führt unabhängig von allen anderen Kantonalbanken ein neues Produkt Deiner Fondsgesellschaft mit einer Wahrscheinlichkeit von 25 Prozent ein.

- Die Produktpolitik der Kantonalbanken ist durch ein komplexes System, welches Du auf der nächsten Seite kennenlernen wirst, miteinander verbunden. Nimmt eine Kantonalbank einen neuen Fonds in ihr 
Sortiment auf, so werden diejenigen Banken, deren Produktpolitik direkt von dieser Bank beeinflusst wird diesen Fonds mit einer Wahrscheinlichkeit von 80 Prozent ebenfalls in ihr Sortiment aufnehmen.

Die Produktpolitik der einzelnen Kantonalbanken ist folgendermaßen miteinander verbunden (Bitte denke daran, dass die Beeinflussung mit einer Wahrscheinlichkeit von 80 Prozent erfolgt) :

- Die Kantonalbank Zürich beeinflusst die Produktpolitik der Kantonalbank Lausanne

- Die Kantonalbank Zürich beeinflusst die Produktpolitik der Kantonalbank Basel

- Die Kantonalbank Lausanne beeinflusst die Produktpolitik der Kantonalbank Neuchâtel

- Die Kantonalbank Lausanne beeinflusst die Produktpolitik der Kantonalbank Genève

- Die Kantonalbank Lausanne beeinflusst die Produktpolitik der Kantonalbank Luzern

- Die Kantonalbank Basel beeinflusst ebenfalls die Produktpolitik der Kantonalbank Luzern

- Die Kantonalbank Basel beeinflusst die Produktpolitik der Kantonalbank Winterthur

- Die Kantonalbank Winterthur beeinflusst die Produktpolitik der Kantonalbank St. Gallen

- Die Kantonalbank St.Gallen beeinflusst die Produktpolitik der Kantonalbank Chur

- Des Weiteren gibt es keinerlei Einflüsse zwischen den Banken

Bitte nimm Dir genügend Zeit, um zu verstehen, wie die Banken miteinander vernetzt sind. Es ist für den weiteren Verlauf des Experimentes unerlässlich, dass Du die Zusammenhänge komplett verstanden hast.

Wie Du auf der letzten Folie gesehen hast, wird die Produktpolitik der Kantonalbank Luzern sowohl von der Kantonalbank Lausanne als auch von der Kantonalbank Basel beeinflusst. Die Ursache hierfür liegt in der geographischen Lage Luzerns, das direkt auf der Grenze der Einflussbereiche der Baseler und Lausanner Banken liegt.

Aufgrund der auf den letzten Folien geschilderten Beziehungen und Wahrscheinlichkeiten wird ein neuer Fonds, der von der Züricher Kantonalbank angeboten wird auch von den meisten anderen Kantonalbanken angeboten.

Ein Fonds, der zumindest von der Kantonalbank Lausanne angeboten wird, wird dann mit hoher Wahrscheinlichkeit auch von den Banken in Neuchâtel, Genève und Luzern angeboten.

Ein Fonds, der nur von der Kantonalbank Basel angeboten wird, wird dann auch mit hoher Wahrscheinlichkeit in Luzern und Winterthur, wegen Winterthur in St. Gallen und dann am Ende in Chur angeboten.

Leider hast Du als Fonds-Verkäufer keinen Einfluss auf die Produktpolitik in Zürich, da diese Kantonalbank vollkommen unabhängig über die Fonds entscheidet. Du hast aber großen Einfluss auf die Produktpolitik der Banken in Basel und Lausanne. In den Fällen, in denen die Kantonalbanken Lausanne und 
Basel einen neuen Fonds nicht anbieten, kommst Du ins Spiel: Du hast die Möglichkeit, einer dieser beiden Kantonalbanken eine äußerst attraktive Gewinnbeteiligung zu gewähren. Dies führt dazu, dass diese Bank den neuen Fonds mit 100\%-iger Wahrscheinlichkeit in ihr Sortiment aufnehmen wird.

Deine Aufgabe wird daher sein, zu entscheiden, welcher der beiden Banken (Lausanne oder Basel) die Gewinnbeteiligung gewährt wird. Wie bereits erwähnt bemisst sich Deine Provision danach, wie viele der neun Kantonalbanken den neuen Fonds anbieten. Hierbei ist einzig und allein die Anzahl der Banken relevant. Daher sollte Deine Entscheidung davon abhängen, wie viele Banken durch deine Entscheidung zusätzlich dazu gebracht werden, den Fonds anzubieten.

So, jetzt hast Du alle Informationen erhalten, die Du benötigst, um als Fondsverkäufer in der Schweiz tätig zu werden. Für die Multiple-Choice-Fragen ist es vor allem wichtig, dass Du folgende Dinge verstanden hast:

- Welche Bank beeinflusst welche andere(n) Bank(en)?

- Wie hoch sind die verschiedenen Wahrscheinlichkeiten dafür, dass

- $\quad$ eine Bank eine andere erfolgreich beeinflusst

- $\quad$ eine Bank einen neuen Fonds unabhängig von der Beeinflussung durch eine andere Bank in ihr Angebot aufnimmt?

- $\quad$ Du selbst eine der Banken davon überzeugst, einen neuen Fonds in ihr Sortiment aufzunehmen?"

Die obige Darstellung entspricht der Balancierungsbedingung 1 in Experiment 4. In Balancierungsbedingung 2 wurden einige der kausalen Beziehungen modifiziert, um geographische Vorwissenseffekte bezüglich der Lage der verschiedenen Orte kontrollieren zu können (s. Anhang E). Eine mögliche graphische Darstellung des sich aus den obigen Instruktionen ergebenden Kausalsystems zeigt Abbildung 23 a) für Balancierungsbedingung 1 und in Abbildung $23 \mathrm{~b}$ ) für Balancierungsbedingung 2. Es wird an dieser Stelle nochmals darauf hingewiesen, dass Abbildung 23 den Versuchspersonen im Experiment nicht zur Verfügung stand.

Wie aus Abbildung 23 ersichtlich, beinhaltete das in Experiment 4 verwendete Kausalsystem sowohl Substrukturen, bei denen eine Variable mehrere potenzielle Ursachen hatte (Common-Cause-Struktur), als auch solche bei denen eine Variable mehrere Effekte hatte (Common-Effect-Struktur). Zudem stellte eine Substruktur eine kausale Kette (Causal Chain) dar. Dadurch ergab sich ein relativ hoher Grad an Komplexität für das in Experiment 4 verwendete Kausalsystem. 


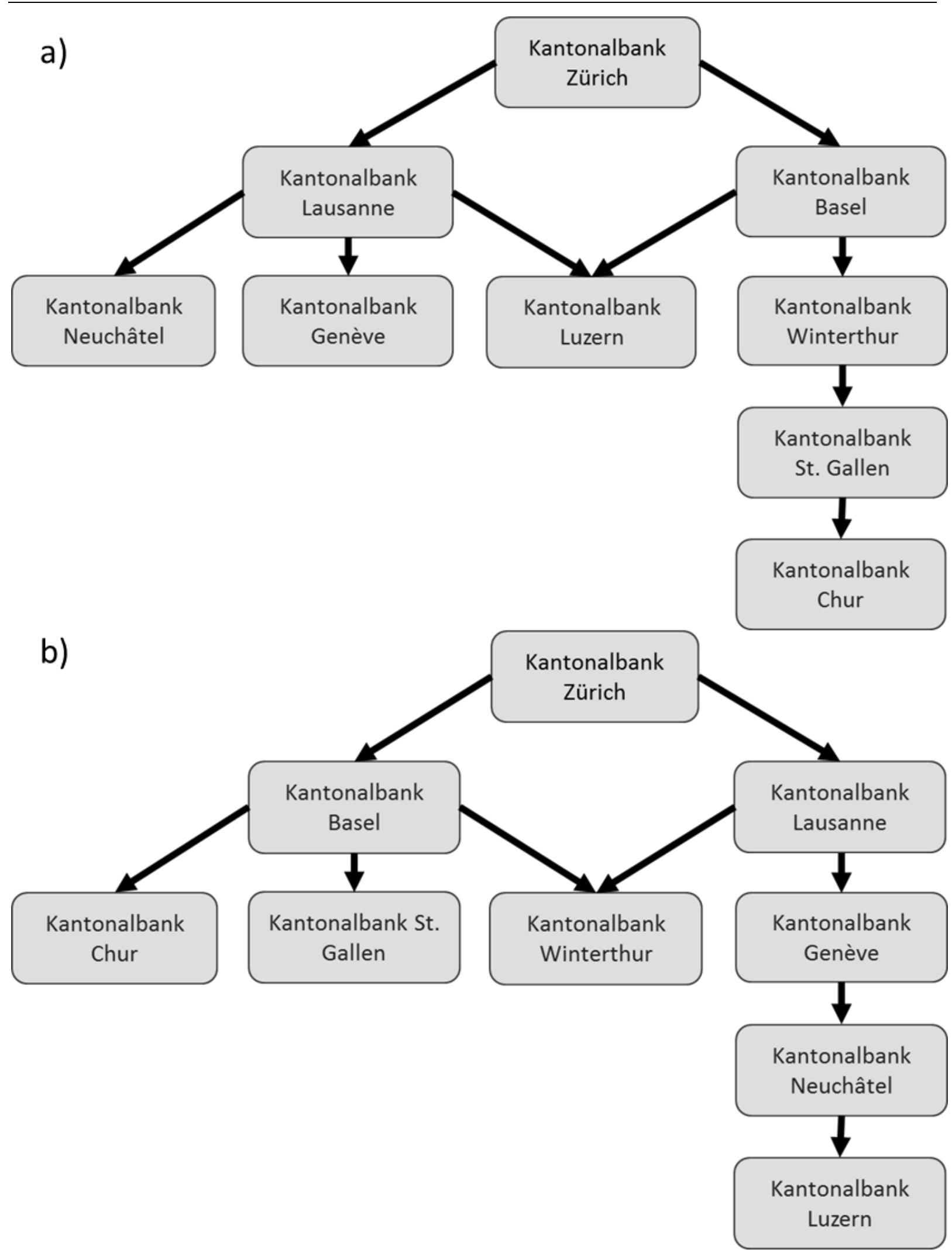

Abbildung 23: Schematische Darstellung des in Experiment 4 benutzten Kausalsystems in Balancierungsbedingung 1 (a) und Balancierungsbedingung 2 (b). Die Pfeile zwischen den Variablen repräsentieren Kausalrelationen und sind von der verursachenden Variablen auf den jeweiligen Effekt gerichtet. Die Ausprägungen der einzelnen Variablen repräsentierten, ob die jeweilige Bank den aktuell präsentierten Aktienfonds im Sortiment hat oder nicht. 


\subsubsection{Multiple-Choice Wissenstest}

Der im Anschluss an die Instruktion des kausalen Systems durchgeführte MultipleChoice-Wissenstest umfasste 12 Fragen mit je 4 Antwortmöglichkeiten, von denen jeweils eine korrekt war (s. Anhang F). Aus der in Experiment 4 vorgenommenen Balancierung ergab sich die Notwendigkeit, die Fragen des Wissenstests entsprechend anzupassen, so dass es für jede Balancierungsbedingung spezifische Multiple-Choice-Fragen gab. Wie in den übrigen im Rahmen dieser Arbeit vorgestellten Experimenten hatte eine Versuchsperson alle Fragen des Wissenstests korrekt zu beantworten, um in die Testphase des Experimentes zu gelangen. Beging sie hingegen einen oder mehrere Fehler, so wurden die Instruktionen erneut präsentiert und der Wissenstest musste nochmals durchlaufen werden. Nach sechsmaliger Durchführung des Tests mit je mindestens einer inkorrekten Antwort wurde einer Versuchsperson zwar die Testphase präsentiert, der entsprechende Datensatz aber als ungültig markiert.

\subsubsection{Testphase}

In der Testphase von Experiment 4 hatte jede Versuchsperson sieben Fälle in randomisierter Reihenfolgt hinsichtlich der Güte zweier möglicher Interventionen in das während der Lernphase instruierte Kausalsystem zu beurteilen. In jedem Durchgang wurde ihr nach der Präsentation der Test-Instruktionen (vgl. Abschnitt 4.1) die später zu beantwortende Frage für ca. 3 Sekunden dargeboten. Der Wortlaut der Frage wurde in Experiment 4 in 2 Stufen balanciert. Balancierung 1 lautete: "Sollte die Kantonalbank Lausanne oder die Kantonalbank Basel beeinflusst werden?“ Für diese Balancierung war der linke Pol (-5) der elfstufigen Ratingskala (die wie in den Experimenten 2 und 3 aus elf Schaltflächen bestand, die mit in 1er Schritten aufsteigenden Zahlenwerten von -5 bis +5 beschriftet waren) mit „sicher Lausanne“ und der rechte Pol (+5) mit „sicher Basel“ gekennzeichnet. In der zweiten Stufe der Balancierung lautete die Frage: „Sollte die Kantonalbank Basel oder die Kantonalbank Lausanne beeinflusst werden?“ Hier war der linke Pol der Antwortskala mit „sicher Basel“ und der rechte Pol mit „sicher Lausanne“ gekennzeichnet. Die nach der Darbietung der Frage erfolgende Stimuluspräsentation erfolgte in Experiment 4 ebenso wie die Vermittlung des Kausalsystems in Textform. Dabei wurde für jede Kantonalbank angezeigt, ob diese den aktuell zu beurteilenden Fonds im Sortiment führt oder nicht. Für die sieben die Testfälle repräsentierenden Fonds wurden fiktive Namen („ProfitMaxx“, „InvestorsChoice“, „MoneyTrust“, „ChinaFirst“”, „EuroInvest“”, „GlobalMoney“ und „SaveHedge“) verwendet, die für alle Versuchspersonen in der gleichen Reihenfolge 
verwendet wurden (d.h. der in Testdurchgang 3 präsentierte Fall wurde den Versuchspersonen immer als der Fonds „MoneyTrust“ vorgestellt). Um die für das Lesen der Stimuli benötigte Zeit zu minimieren wurde darüber hinaus nur der Name der Städte, in denen sich die Kantonalbanken befinden, angezeigt. Den Versuchspersonen wurden also beispielsweise die Sätze „Lausanne hat ProfitMaxx im Sortiment“ oder „Winterthur hat ProfitMaxx NICHT im Sortiment“ präsentiert. Das Wort „NICHT“ wurde auch im Experiment durch die Verwendung von Großbuchstaben hervorgehoben, um Überlesen zu verhindern. Die Darbietungszeit jeder der insgesamt 9 Variablenausprägungen pro Fall betrug 2,5 Sekunden und war damit eine Sekunde länger als in den übrigen im Rahmen dieser Arbeit vorgestellten Experimenten. Die längere Präsentationsdauer wurde gewählt, weil Vortests gezeigt hatten, dass eine Aufnahme der Information in 1,5 Sekunden nicht fehlerfrei möglich war. Dies ist möglicherweise dadurch bedingt, das zwei Informationen (Ort und Fondsname) zu verarbeiten waren.
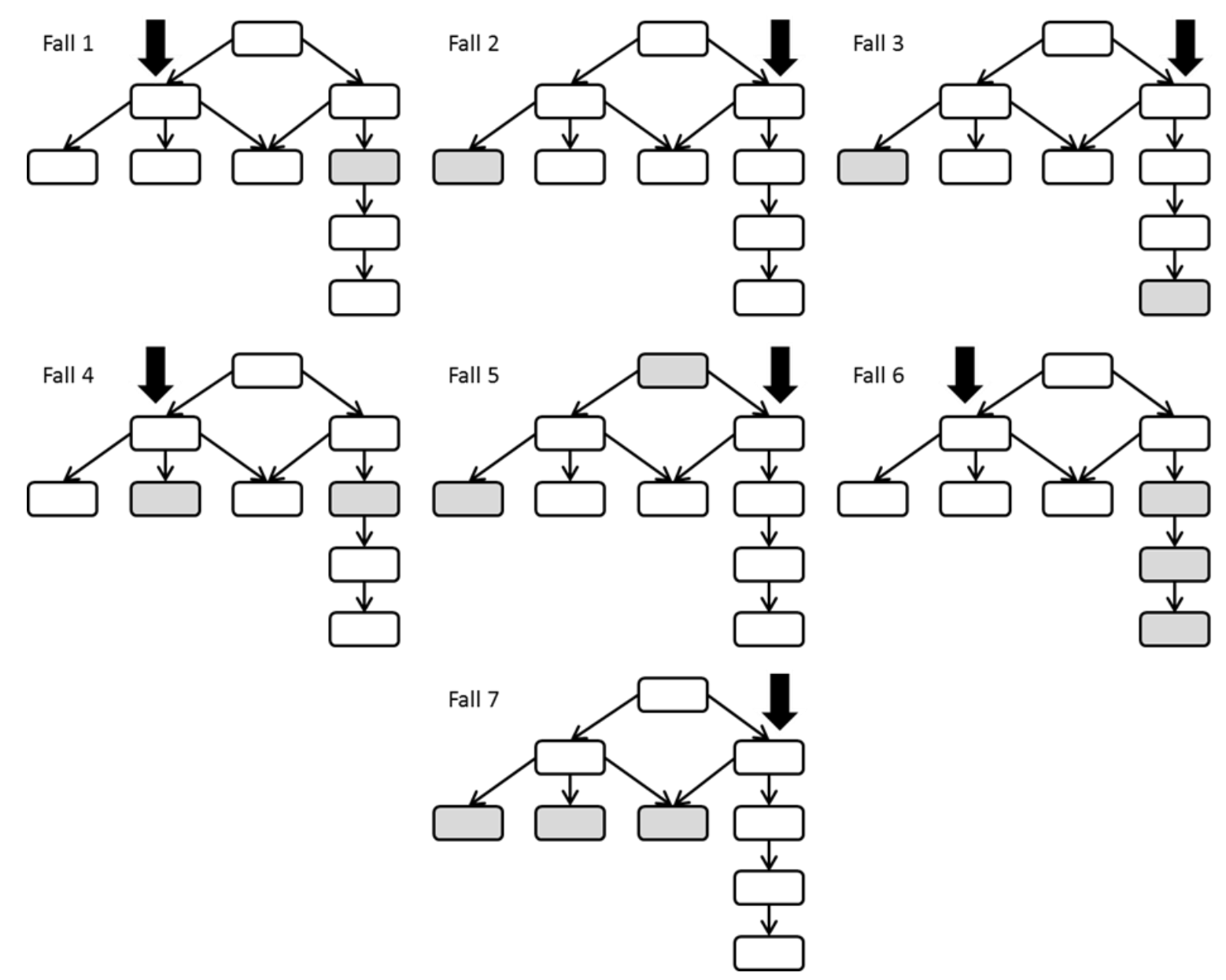

\footnotetext{
Abbildung 24: In Experiment 4 verwendete Fälle. Die Felder stellen die verschiedenen Variablen (Kantonalbanken) dar, die Pfeile repräsentieren die Kausalbeziehungen zwischen den Variablen, wobei ein Pfeil immer von einer Ursache auf einen Effekt weist. Grau gefärbte Felder zeigen an, welche der Banken den jeweiligen Aktienfonds vor der Intervention bereits in ihr Sortiment aufgenommen hat. Die schwarzen Felder in Pfeilform zeigen den Ort der effektivsten Intervention für den jeweiligen Fall an (links: Intervention A; rechts: Intervention B).
} 
Im Anschluss an die Stimuluspräsentation hatten die Versuchspersonen in der Bedingung des unbewussten Denkens und der Bedingung des bewussten Denkens mit festgelegter Dauer die in Abschnitt 4.1 erläuterten Aufgaben zu absolvieren. Danach wurde unmittelbar die $\mathrm{zu}$ beantwortende Frage zusammen mit der bekannten elfstufigen Antwortskala eingeblendet, auf der sie innerhalb von 3 Sekunden ihr Urteil abzugeben hatten. Versuchspersonen, die länger als die vorgesehenen 3 Sekunden für ihre Antwort benötigten, wurde direkt nach Abgabe ihrer Einschätzung ein Hinweis präsentiert, der sie aufforderte, beim nächsten Mal schneller zu antworten. Nach Schließen des Fensters wurde automatisch der nächste Testfall präsentiert.

Die den Versuchspersonen in Experiment 4 präsentierten Fälle sind in Abbildung 24 graphisch dargestellt. Dabei wurde auch die jeweils effektivere Intervention hervorgehoben. Die Variablenausprägungen der einzelnen Fälle wurden so gewählt, dass die auf Basis des instruierten Kausalsystems richtige Intervention nicht von den instruierten Parametern (Basisraten und Kausalstärken) abhing, sondern durch einfaches Abzählen der durch eine Intervention beeinflussbaren Variablen bestimmt werden konnte. Gemäß der aufgestellten Hypothese 1 wird davon ausgegangen, dass die Versuchspersonen durch das Wirken von Typ 2-Verarbeitungsprozessen in der Lage sein sollten, die gemäß dem instruierten Kausalsystem effektivere der beiden möglichen Interventionen ausfindig $\mathrm{zu}$ machen. Für Balancierungsbedingung 1 bedeutet dies, dass es in drei Fällen (1, 4 und 6) effektiver ist, die Kantonalbank in Lausanne dazu zu bringen, den Fonds in ihr Sortiment aufzunehmen. In den Fällen 1 und 6 kann eine Intervention auf die Kantonalbank Lausanne maximal 4 Banken beeinflussen, während durch eine Intervention auf die Kantonalbank Basel maximal 2 Banken beeinflusst werden können. In Fall 4 beträgt das Verhältnis der maximal zu beeinflussenden Banken bei einer Intervention in Lausanne bzw. Basel 3:2. Hingegen ist es in den übrigen vier Fällen (2, 3, 6 und 7) besser, die Kantonalbank Basel hinsichtlich der Aufnahme des Fonds in ihr Angebot zu beeinflussen. In Fall 7 können durch eine Intervention in Basel maximal 4 Banken, durch eine Intervention in Lausanne 1 Bank beeinflusst werden. Für die Fälle 2 und 5 ergibt sich ein Verhältnis von 5:3, für Fall 3 ein Verhältnis von 4:3 für eine Intervention in Basel. Für Balancierungsbedingung 2 ergibt sich spiegelbildlich, dass für die Fälle 1, 4 und 6 eine Beeinflussung der Kantonalbank Basel und für die Fälle 2, 3, 6 und 7 eine Beeinflussung der Kantonalbank Lausanne effektiver ist. 


\subsubsection{Ergebnisse}

Die Daten von 50 der ursprünglichen 86 Versuchspersonen gingen in die in den folgenden Abschnitten vorgestellte Analyse der Ergebnisse von Experiment 4 ein. Hiervon waren $23(46 \%)$ weiblich und 9 (18\%) Studierende der Psychologie. Die relativ niedrigen Anteile weiblicher Probanden und Psychologiestudierender lassen sich auf den Zeitpunkt der Durchführung des Experiments während der Semesterferien zurückführen, in denen ein hoher Anteil der (vorwiegend weiblichen) Psychologiestudierenden erfahrungsgemäß nicht in Göttingen ist. Der relativ hohe Verlust an potentiellen Daten erklärt sich zum einen durch 12 Versuchspersonen, bei denen das Experiment aufgrund von technischen Schwierigkeiten bzw. Abbrüchen durch die Versuchspersonen bereits vor Abschluss des Multiple-Choice Wissenstests beendet wurde. Weitere 21 Versuchspersonen scheiterten am Multiple-Choice Wissenstest zum instruierten Kausalsystem. 3 Versuchspersonen brachen das Experiment nach erfolgreichem Absolvieren des Wissenstests ab. Von den verbleibenden 50 Versuchspersonen entfielen 15 auf die Bedingung spontanen Entscheidens, 17 auf die Bedingung unbewussten Denkens und 18 auf die Bedingung bewussten Denkens mit festgelegter Dauer. Diese Versuchspersonen benötigten durchschnittlich 47 Minuten für die Durchführung des Experimentes (spontanes Entscheiden: 34 Minuten; unbewusstes Denken: 55 Minuten; bewusstes Denken: 50 Minuten).

Für die Analyse wurden die Daten zunächst so transformiert, dass niedrige Ratings einer Präferenz für eine Intervention bei der Kantonalbank Lausanne in Balancierungsbedingung 1 (im Folgenden: Intervention A) entsprechen. Hohe Ratings entsprechen demgegenüber einer Präferenz für eine Intervention bei der Kantonalbank Basel in Balancierungsbedingung 1 (im Folgenden: Intervention B). Im Anschluss wurden die Ratings auf Ebene der Versuchspersonen für Fälle mit gleichem erwartetem Urteil (vgl. Abschnitt 4.5.3.3) gemittelt, so dass insgesamt 5 Fall-Cluster gebildet wurden.

\subsubsection{Multiple-Choice Wissenstest}

21 von 74 Versuchspersonen konnten innerhalb der maximal erlaubten 6 Durchgänge nicht alle 12 Fragen des Multiple-Choice Wissenstests korrekt beantworten. Von diesen 21 Versuchspersonen entfielen 9 auf die Bedingung spontanen Entscheidens und jeweils 6 auf die Bedingungen unbewussten bzw. bewussten Denkens. Die relativ hohe Ausfallquote von $28 \%$ kann auf verschiedene mögliche Ursachen zurückgeführt werden. Zum einen wurden die Instruktionen ausschließlich in Textform präsentiert, was das Lernen erschwert haben kann. 
Ein weiteres Indiz für diese Überlegung ist eine im Vergleich zu den anderen Experimenten höhere Anzahl von Lerndurchgängen (im Durchschnitt 3,28), die diejenigen Versuchspersonen benötigten, die den Test letztlich erfolgreich absolvieren konnten (s. Abbildung 25). Es ließ sich kein signifikanter Einfluss der Verarbeitungsbedingung auf die Anzahl der im Multiple-Choice-Wissenstest benötigten Durchgänge feststellen $(F(2,50)<1$, $p=0,78, M S E<1)$. Ein weiterer Grund für die relativ hohe Anzahl an durchschnittlich benötigten Lerndurchgängen könnte in der im Vergleich $\mathrm{zu}$ den anderen Experimenten höheren Anzahl von 12 Multiple-Choice Fragen liegen. Außerdem besaß das beschriebene Kausalsystem verschiedenartige Substrukturen (gemeinsame Ursachen, gemeinsame Effekt, kausale Ketten) und dadurch eine relativ hohe Komplexität.

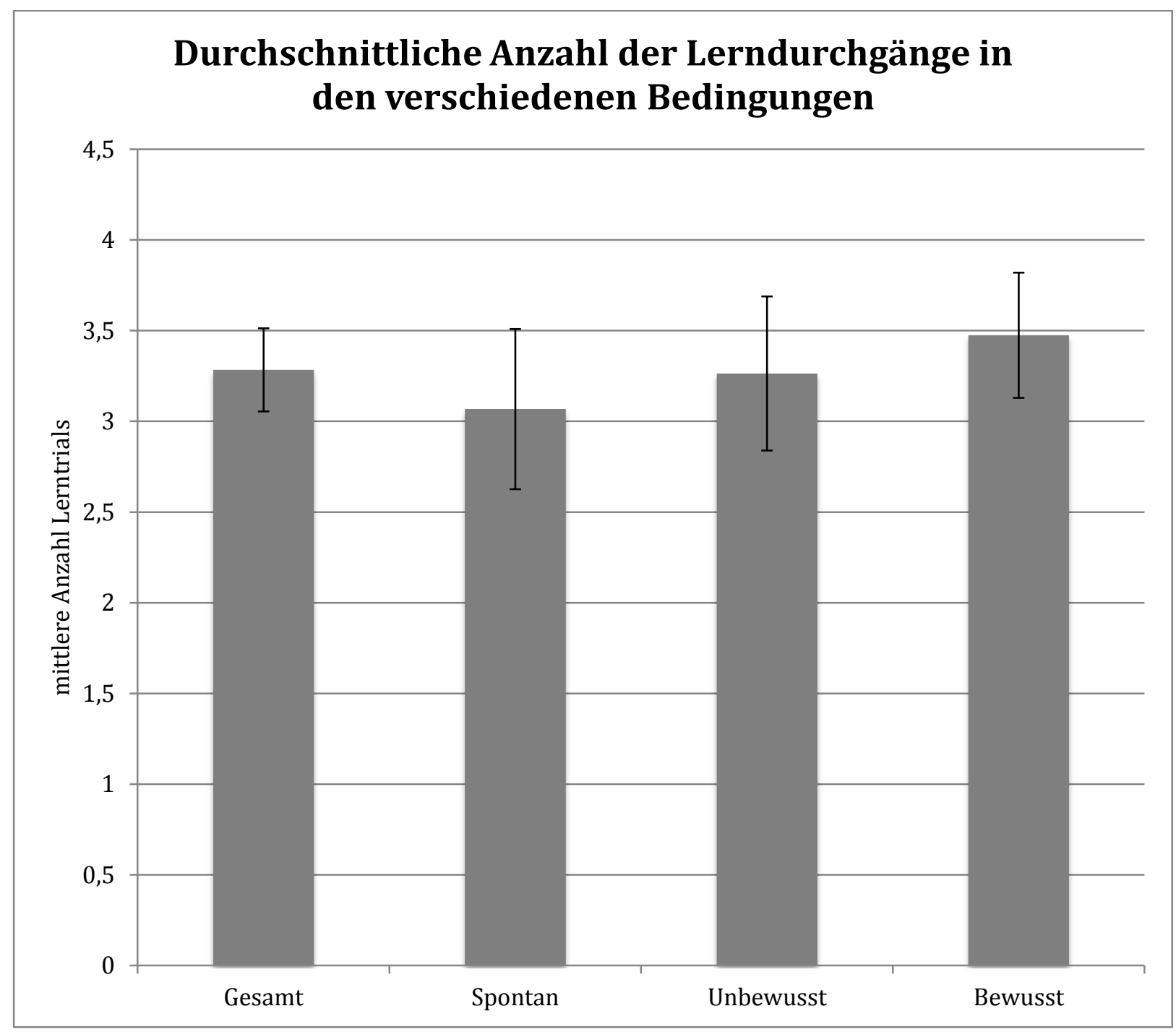

Abbildung 25: Durchschnittliche Anzahl der in Experiment 4 in den verschiedenen Verarbeitungsbedingungen benötigten Durchgänge (und deren Standardfehler) beim Multiple-Choice Wissenstest. Die Datengrundlage bilden Versuchspersonen, die innerhalb von 6 Durchgängen alle Fragen korrekt beantworten konnten. 


\subsubsection{Interventionsurteile}

Zunächst wurden die Muster von Antworten, die innerhalb von 3 bzw. 5 Sekunden nach Erscheinen der Antwortskala abgegeben wurden, mittels zweifaktoriellen Varianzananlysen mit Messwiederholungen auf Gleichartigkeit geprüft. Für beide Intervalle wurde ein signifikanter Effekt der Fall-Cluster (3 Sekunden: $F(4,120)=6,01, p<0,001$, $M S E=6205 ; 5$ Sekunden: $F(4,164)=7,08, \quad p<0,001, M S E=7402)$, sowie eine marginal signifikante Interaktion zwischen den Verarbeitungsbedingungen und den Fall-Clustern (3 Sekunden: $\quad F(8,120)=1,84, p=0,08, \quad M S E=1895,9 ; 5$ Sekunden: $\quad F(8,164)=1,73, \quad p=0,10$, $M S E=1806)$ festgestellt. Hingegen zeigte die Variation der Verarbeitungsbedingungen (3 Sekunden: $F(2,30<1, p=0,63, M S E=302 ; 5$ Sekunden: $F(2,41)=1,11, p=0,34, M S E=577)$ keinen Effekt. Die Ersetzung der fehlenden Werte für die Daten auf Basis des 5-Sekunden Intervalls mit den über alle Bedingungen gemittelten Ratings für die entsprechenden FallCluster resultierte in einem signifikanten Effekt für die Fall-Cluster $(F(4,188)=8,42, p<0,001$, $M S E=8469)$, während die Variation der Verarbeitungsbedingung $(F(2,47)=1,07, p=0,35$, $M S E=517)$ und die Interaktion zwischen Verarbeitungsbedingungen und Fall-Clustern $(F(8,188)=1,21, p=0,30, M S E=1211)$ keine signifikanten Effekte nach sich zogen. Aufgrund der relativ deutlichen Veränderung in Bezug auf die Interaktion wird die folgende Kontrastanalyse (s. Tabelle 7) auf Grundlage der Daten ohne Ersetzung der fehlenden Werte durchgeführt (s. Abbildung 26) für eine graphische Darstellung der

Tabelle 7: Kontraste zwischen Fall-Clustern mit unterschiedlichen erwarteten Interventionssurteilen in den verschiedenen Verarbeitungsbedingungen von Experiment 4

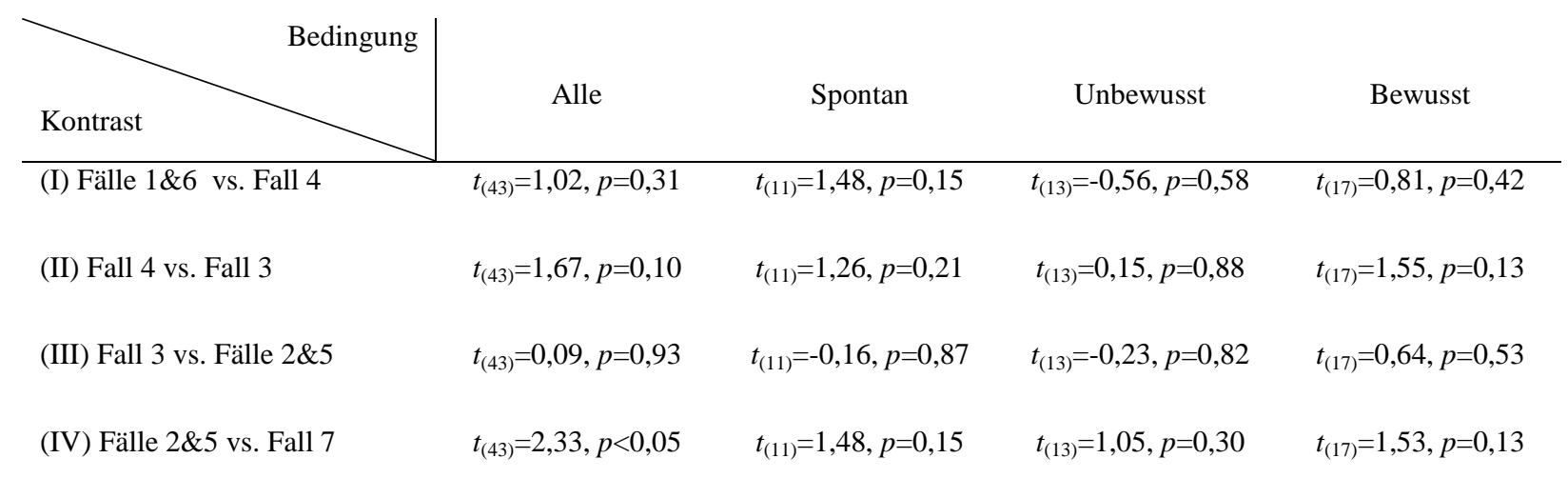

Urteile in den verschiedenen Verarbeitungsbedingungen). Die Ergebnisse der Analyse der Kontraste zwischen den Fall-Clustern ergab trotz des in der Varianzanalyse gefundenen globalen Effekts der Fall-Cluster in keiner der drei Verarbeitungsbedingungen einen signifikanter Unterschied zwischen zwei benachbarten Fall-Clustern. Wie die Betrachtung der 
Bedingung spontanen Entscheidens sowie der Bedingung bewussten Denkens in Abbildung 26 nahelegt, könnte dieses Ergebnis durch die relativ geringe Versuchspersonenzahl und die daraus resultierende geringe Teststärke begründet sein. Um zu prüfen, ob die Versuchspersonen zumindest zwischen Fällen mit unterschiedlichen zu präferierenden Interventionen differenzierten, sollen die Fälle nun ausschließlich gemäß der präferierten Intervention zusammengefasst werden. Die dadurch entstehenden zwei Fall-Cluster umfassen die Fälle 1, 4 und 6, sowie die Fälle 2, 3, 5 und 7 und bilden die Basis der folgenden Analysen.

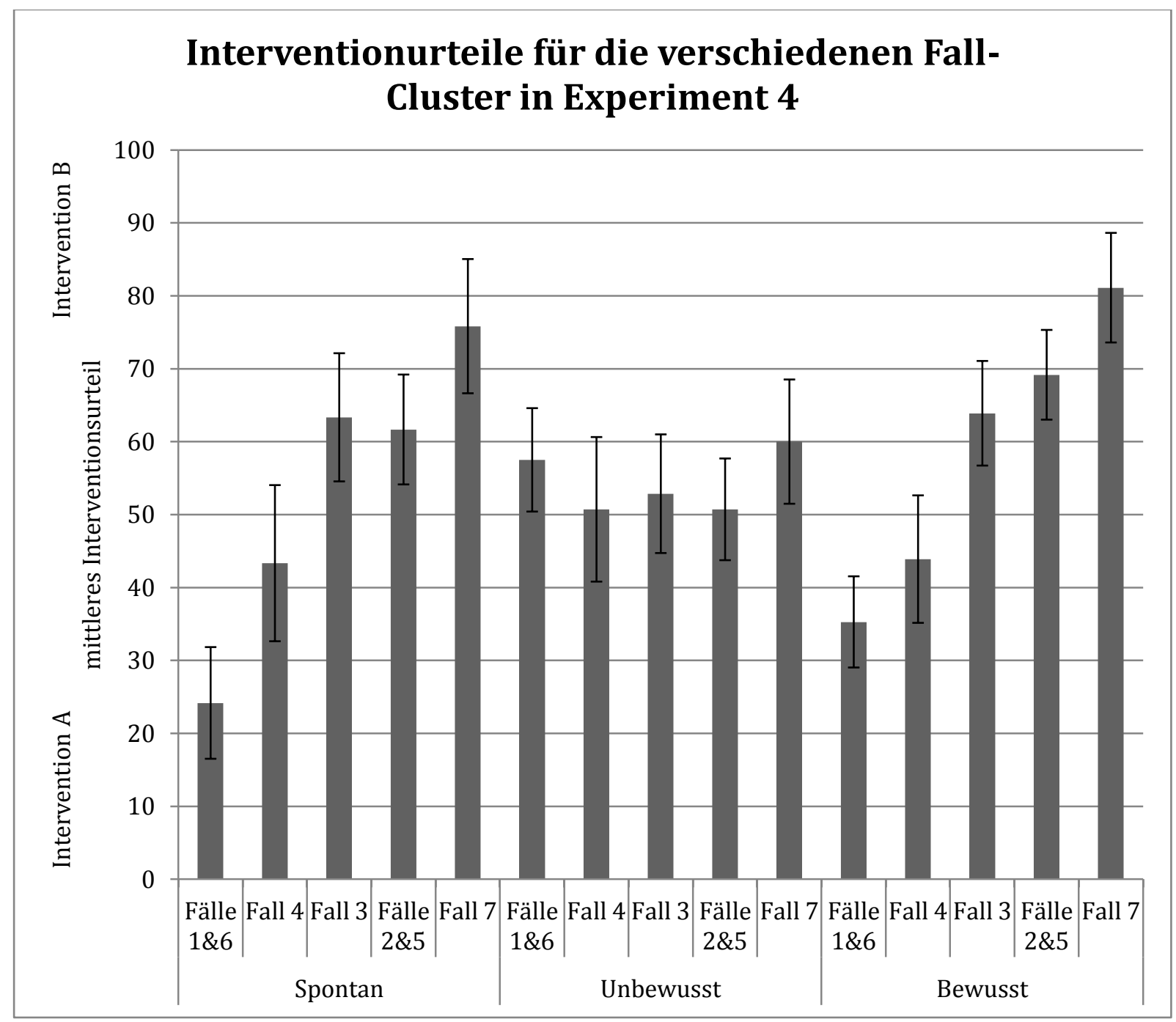

Abbildung 26: Interventionsurteile (und Standardfehler) für nach erwartetem Rating gebildete Fall-Cluster in den verschiedenen Verarbeitungsbedingungen von Experiment 4.

Zunächst wurde wieder die Gleichartigkeit der Muster für Antworten, die 3 bzw. 5 Sekunden nach Erscheinen der Antwortskala abgegeben wurden überprüft. In beiden Fällen ergab sich ein signifikanter Einfluss der Fall-Cluster (3 Sekunden: $F(1,47)=16,53, p<0,001$, $M S E=12810$; 5 Sekunden: $F(1,47)=16,75, p<0,001, M S E=12720)$, eine marginal signifikante 
Interaktion zwischen Verarbeitungsbedingung und Fall-Clustern (3 Sekunden: $F(2,47)=2,95$, $p=0,06, M S E=2287 ; 5$ Sekunden: $F(2,47)=2,44, p=0,10, M S E=1855)$ und kein Einfluss der Verarbeitungsbedingung (3 Sekunden: $F(2,47)<1, p=0,42, M S E=216$; 5 Sekunden: $F(2,47)<1$, $p=0,51, M S E=109)$. Da die Daten für Urteile, die innerhalb von 5 Sekunden abgegeben wurden zudem keine fehlenden Werte enthielten, bilden sie die Grundlage der folgenden Kontrastanalysen und sind in Abbildung 27 graphisch veranschaulicht.

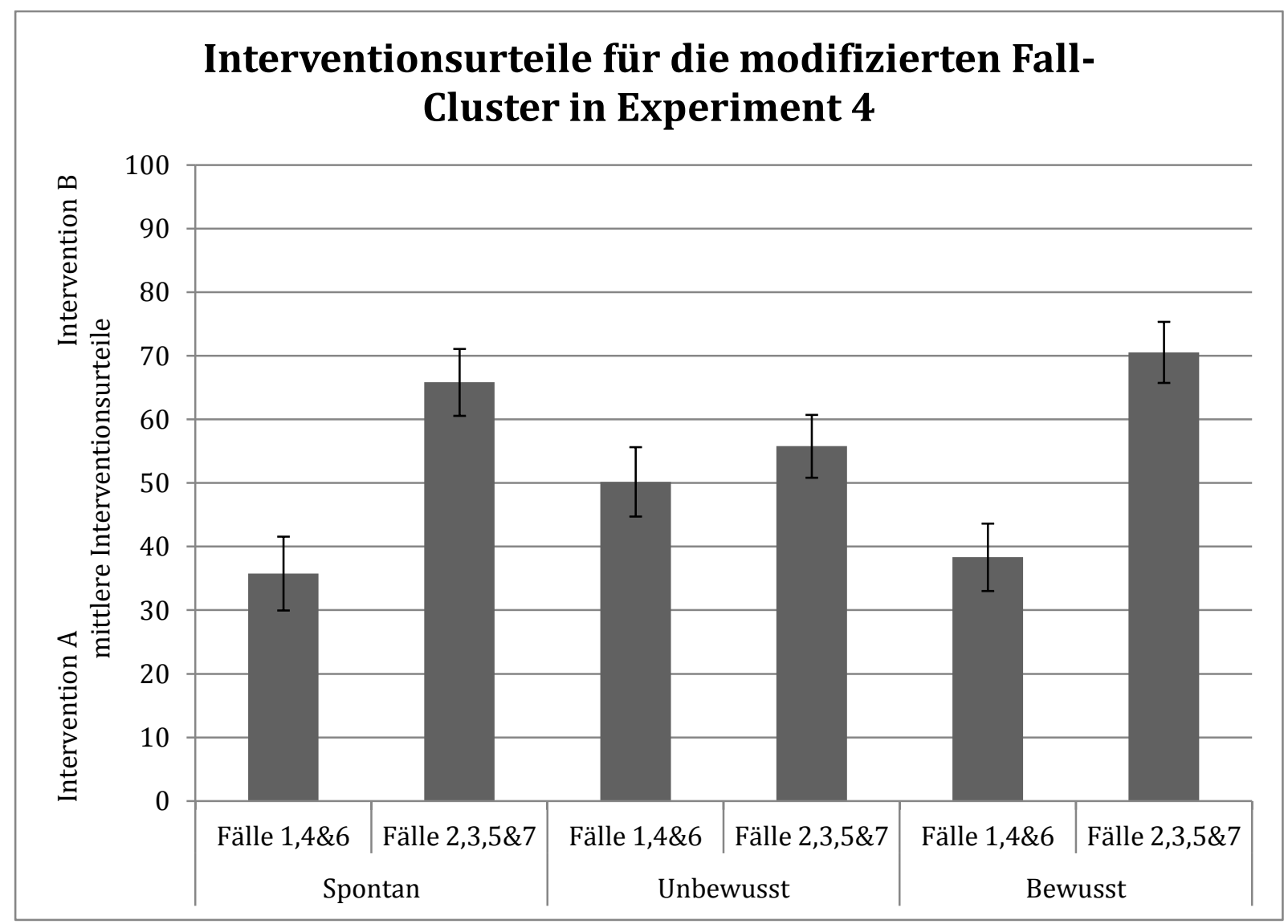

Abbildung 27: Interventionsurteile (und Standardfehler) für nach $\mathrm{zu}$ präferierender Intervention zusammengefasste Fälle in den verschiedenen Verarbeitungsbedingungen von Experiment 4.

Die Versuchspersonen konnten über alle Bedingungen betrachtet zwischen den beiden Fall-Clustern unterscheiden $\left(t_{(49)}=4,09, p<0,001\right)$. Bei genauerer Analyse der verschiedenen Verarbeitungsbedingungen zeigt sich, dass diese Differenzierungsleistung von Versuchspersonen in den Bedingungen spontanen Entscheidens $\left(t_{(14)}=2,99, p<0,01\right)$, sowie des Entscheidens nach einer Phase bewussten Nachdenkens $\left(t_{(17)}=3,51, p<0,01\right)$ erbracht wurde. Versuchspersonen in der Bedingung unbewussten Denkens differenzierten hingegen nicht zwischen den beiden Fall-Clustern $\left(t_{(16)}=0,59, p=0,56\right)$. Darüber hinaus zeigte sich, dass die Differenzierungsleistung in der Bedingung bewussten Denkens signifikant besser war als in der Bedingung unbewussten Denkens $\left(t_{(33)}=2,02, p<0,05\right)$. Der Unterschied zwischen der Bedingung spontanen Entscheidens und der Bedingung unbewussten Denkens war marginal 
signifikant $\left(t_{(30)}=1,77, p=0,08\right)$. Zwischen der Bedingung bewussten Denkens und spontanen Entscheidens konnte entgegen der für Interventionsentscheidungen aufgestellten Hypothese kein Unterschied festgestellt werden $\left(t_{(31)}=0,16, p=0,87\right)$.

\subsubsection{Diskussion}

Die in Experiment 4 überprüfte Hypothese 2 konnte auf Grundlage der Ergebnisse nicht bestätigt werden. Die Analyse der Entscheidungen der Versuchspersonen in Experiment 4 führte zu dem überraschenden Ergebnis, dass Versuchspersonen bereits unmittelbar nach der Präsentation der Stimuli zu Interventionsurteilen kommen, die mit einem zuvor gelernten komplexen Kausalsystem in Einklang stehen. In der Typ 2-Verarbeitungsprozesse ermöglichende Bedingung bewussten Nachdenkens, die Typ 2-Verarbeitungsprozesse ermöglichte, waren sie dazu zwar ebenfalls in der Lage, die vorhergesagte Überlegenheit gegenüber der Bedingung spontanen Entscheidens konnte jedoch nicht festgestellt werden. Eine mögliche Erklärung für die gute Leistung in der Bedingung spontanen Entscheidens liegt in der Veränderung der Dauer der Stimuluspräsentation. Während die einzelnen Variablenausprägungen in Experiment 1 für jeweils 1,5 Sekunden präsentiert wurden, betrug die Präsentationsdauer in Experiment 2 jeweils 2,5 Sekunden. Der Grund hierfür (vgl. Abschnitt 4.5.3.3) liegt darin, dass die Variablenausprägungen in Experiment 2 in Textform eingeblendet wurden (z.B. „Die Kantonalbank Winterthur hat den Fonds ProfitMaxx NICHT im Angebot"“). Vortests ergaben, dass eine Präsentationsdauer von 1,5 Sekunden von den Probanden als zu kurz empfunden wurde und eine Dauer von 2,5 Sekunden als angemessen erschien, um die Information aufnehmen zu können. Da es aber während des Experiments aufgrund der pro Fonds geblockten Darstellung ausreicht, den Namen der Stadt sowie die Information ,im Angebot“ vs. „NICHT im Angebot“ wahrzunehmen, kann die gewählte Dauer von 2,5 Sekunden zusätzliche Online-Verarbeitung und damit Typ 2-Prozesse ermöglicht haben. Diese war offensichtlich ausreichend, um $\mathrm{zu}$ den korrekten Interventionsurteilen zu kommen.

Der Umstand, dass Versuchspersonen in der Bedingung unbewussten Denkens nicht zwischen den Fall-Clustern mit unterschiedlichen erwarteten Ratings differenzieren konnten, schlägt sich auch in einer signifikant besseren Differenzierungsleistung der Versuchspersonen in der Bedingung bewussten Denkens, sowie in einer marginal signifikant besseren Differenzierungsleistung der Versuchspersonen in der Bedingung spontanen Entscheidens nieder. Eine mögliche Erklärung hierfür wäre, dass ein während der Stimuluspräsentation gebildetes Urteil zwar sofort oder nach einer Phase bewussten Denkens wiedergegeben 
werden kann, es aber während der 2-Back-Distraktoraufgabe zu Interferenzen im Arbeitsgedächtnis kommt. Dies könnte die Konsequenz haben, dass das Urteil nach der Distraktoraufgabe nicht mehr korrekt erinnert und wiedergegeben werden kann.

\subsection{Experiment 5}

\subsubsection{Ziel des Experiments}

Das im Folgenden vorgestellte Experiment 5 unterscheidet sich in mehreren Punkten von den übrigen im Rahmen dieser Arbeit dargestellten Experimenten. Sowohl der Ablauf als auch die Aufgabenstellung wurde modifiziert, um Bedingungen herzustellen, die einen zum einen einen möglichst guten Test für die in Abschnitt 3.2 vorgestellte Unconscious Thought Theory (UTT; Dijksterhuis \& Nordgren, 2006) auf dem Gebiet kausalen Urteilens und Entscheidens zu ermöglichen. Eines der Prinzipien der UTT besagt, dass unbewusstes Denken umso deutlichere Vorteile gegenüber bewusstem Denken aufweist, je mehr Informationen für eine Entscheidung berücksichtigt werden müssen. Daher könnten Vertreter dieser Theorie argumentieren, dass in den bisher vorgestellten Experimenten aufgrund der geringen Anzahl $\mathrm{zu}$ berücksichtigender Variablenausprägungen $(8$ - 12) ungünstige Bedingungen für unbewusste Denkprozesse geherrscht haben. Darüber hinaus war die Dauer der als Distraktor genutzten 2-back-Aufgabe mit zwei Minuten eher kurz, während eine längere Zeitspanne bessere Resultate des unbewussten Denkens erwarten ließe, da angenommen wird, dass dieser Denkprozess langsam abläuft (vgl. Dijksterhuis \& Nordgren, 2006). Eine weitere Abweichung der bisher vorgestellten eigenen Experimente von den von Dijksterhuis und Kollegen durchgeführten Studien zur UTT besteht in der Art der von den Versuchspersonen $\mathrm{zu}$ bearbeitenden Aufgabe. Während in den eigenen Experimenten Kategorisierungen oder Interventionen beurteilt werden mussten, bestanden die Aufgaben in Studien zur UTT in der Regel aus Bewertungen mehrerer Alternativen im Hinblick auf die Erreichung eines Ziels (vgl. Strick et al., 2011). Des Weiteren soll mit Experiment 5 untersucht werden, ob die Notwendigkeit von Typ 2-Verarbeitungsprozesse bei der Durchführung mentaler Simulationen besteht. Diese stellen einen wichtige Komponenten bei der Abschätzung von Interventionsfolgen dar und sollen daher in Experiment 5 unabhängig vom äußeren Einfluss von Interventionen untersucht werden.

Auf Grundlage dieser Überlegungen wurde Experiment 5 im Vergleich zu den Experimenten 1 bis 4 in mehrfacher Hinsicht modifiziert, wenn auch die generelle Struktur der Übungs-, Lern- und Testphasen beibehalten wurde. Die beiden bedeutendsten 
Veränderungen betrafen zum einen die Präsentation der vier in Experiment 5 verwendeten Fälle. Diese wurden, abweichend von den bisher vorgestellten Experimenten, unmittelbar hintereinander in einem einzigen Versuchsdurchgang präsentiert. Dabei wurden die Variablenausprägungen eines Falles in randomisierter Reihenfolge in einem Block präsentiert.

Die Reihenfolge der Präsentation der verschiedenen Fälle wurde ebenfalls randomisiert. Die Veränderung der Stimuluspräsentation zog auch eine Modifikation der Abfrage der Entscheidung, die wie in den übrigen Experimenten nach Bearbeitung einer bedingungsspezifischen Aufgabe erfolgte, nach sich: Die Versuchspersonen hatten ihre Urteile bezüglich der vier Fälle unmittelbar nacheinander abzugeben, wobei ihnen für jedes ihrer vier Urteile ein Antwortfenster von 3 Sekunden zur Verfügung stand. Die zweite bedeutende Veränderung betraf die Art der $\mathrm{zu}$ bearbeitenden Aufgabe. Die den Versuchspersonen in Experiment 5 präsentierten Fälle stellten vier Instanziierungen der während der Lernphase instruierten Kausalsysteme vor dem Wirken der kausalen Mechanismen dar. Die Aufgabe der Versuchspersonen bestand darin, die Güte der vier präsentierten Fälle nach dem Wirken der kausalen Mechanismen auf der bekannten elfstufigen Antwortskala einzuschätzen. Sie mussten somit auf Grundlage der präsentierten Fälle und ihres Wissens über die existierenden kausalen Mechanismen Vorhersagen über den Endzustand des kausalen Systems treffen, also die kausalen Wirkungen mental simulieren. Die Übungsphase von Experiment 5 wurde so modifiziert, dass sich die Versuchspersonen mit der veränderten Aufgabenstruktur vertraut machen und dadurch auf die spätere Testphase vorbereiten konnten. Die kausale Verarbeitung wurde in Experiment 5 in vier Bedingungen untersucht. Diese waren die Bedingungen des spontanen Entscheidens, des Entscheidens nach einer 4-minütigen Phase unbewussten Denkens, des Entscheidens nach einer 4-minütigen Phase bewussten Denkens, sowie des Entscheidens nach einer Phase bewussten Denkens mit selbstgewählter Dauer.

\subsubsection{Stichprobe und verwendetes Design}

Insgesamt nahmen 101 Versuchspersonen an Experiment 5 teil, die über eine Versuchspersonendatenbank rekrutiert wurden (s. Abschnitt 4.1). Das Experiment war als 4 (Verarbeitungsbedingungen: Spontan vs. Unbewusst vs. Bewusst mit festgelegter Dauer vs. Bewusst mit selbstgewählter Dauer) X 4 Fälle Design konzipiert, bei dem die Variation der Verarbeitungsbedingungen zwischen den Versuchspersonen, die Variation der präsentierten Fälle hingegen innerhalb der Versuchspersonen erfolgte. 


\subsubsection{Ablauf und verwendetes Material}

Die wesentlichen Veränderungen in Experiment 5 wurden bereits weiter oben beschrieben, so dass im Folgenden vor allem auf weitere, kleinere Modifikationen eingegangen werden soll. Zudem soll die Coverstory die in Experiment 5 verwendeten Kausalsystems zusammenfassend vorgestellt und im Wortlaut wiedergegeben werden. Aufgrund der hohen Anforderungen an die Informationsverarbeitung der Versuchspersonen, die durch die Präsentation der Variablenausprägungen einer Sequenz von vier Fällen à 8 Variablen hervorgerufen wird, war das in Experiment 5 verwendete Kausalsystem verhältnismäßig einfach strukturiert. Genau genommen handelte es sich um zwei kausale Strukturen, die jeweils vier Variablen beinhalteten, die mit je drei Kausalrelationen miteinander verbunden waren. Bei der einen Struktur handelte es sich um eine kausale Kette, während die andere Struktur drei von einer gemeinsamen Ursache beeinflusste Effekte darstellte (vgl. Abbildung 28). Die kausalen Beziehungen zwischen den Variablen waren deterministisch. Die kausalen Strukturen wurden den Versuchspersonen mittels einer Coverstory instruiert, in der sich die Versuchsperson in die Rolle eines Mitarbeiters / einer Mitarbeiterin einer Schuhe produzierenden Firma versetzen sollte. Die Aufgabe der Versuchspersonen bestand darin, zu entscheiden, welcher von vier Schuh-Prototypen tatsächlich produziert werden sollte. Dabei sollte die Anzahl von Schuhgeschäften, die bereit waren, die einzelnen Prototypen in ihr Angebot aufzunehmen entscheidend sein. Die Geschäfte waren in die oben erwähnten kausalen Strukturen eingebunden. Der instruierte kausale Mechanismus bestand darin, dass ein Geschäft mit absoluter Sicherheit dafür sorgte, dass ein von ihm angebotenes Schuhmodell auch in allen ihr direkt in der kausalen Struktur untergeordneten Geschäften angeboten werden würde. Die Beschreibung des verwendeten Kausalsystems war in zwei Stufen balanciert, um Effekte der Positionierung der Variablen kontrollieren zu können. Hierzu wurden die Geschäfte in den kausalen Substrukturen getauscht (vgl. Abbildung 28).

Zur Veranschaulichung wird im Folgenden der Wortlaut der Instruktionen für die erste der beiden Balancierungsbedingungen wiedergegeben. Der Wortlaut der Instruktionen für Balancierungsbedingung 2 befindet sich in Anhang G. 


\subsubsection{Instruktionen Kausalsystem Schuhe}

„,Wir kommen nun zum eigentlichen Experiment. Stelle Dir hierzu bitte folgende Situation vor: Du bist MitarbeiterIn einer Firma, die neue Schuhe designt und produziert. Einmal im Quartal werden mehrere Prototypen exklusiver neuer Schuhmodelle von der Design-Abteilung deiner Firma entwickelt. Von diesen Prototypen wird jedoch nur ein Modell ausgewählt, das später tatsächlich produziert und verkauft wird. Natürlich soll genau das Modell produziert werden, von dem sich deine Firma den größten Absatz verspricht. Genau hier kommst du ins Spiel, denn deine Aufgabe besteht darin, zu überprüfen, welcher der Prototypen in den meisten der von deiner Firma belieferten Schuhgeschäfte verkauft werden wird. Um diese Aufgabe zu erfüllen, benötigst du einige Informationen, die dir im Folgenden zur Verfügung gestellt werden:

Die Prototypen werden ausschließlich den Filialen von zwei exklusiven Ketten von Schuhgeschäften vorgestellt. Die ersten Filialen der einen Kette wurden in Berlin gegründet, die der anderen in München. Die Berliner Filiale der ersten Kette eröffnete später drei untergeordnete Filialen in Leipzig, Hamburg und Hannover. Immer wenn die Berliner Filiale ein neues Schuhmodell in ihr Angebot aufnimmt, sorgt sie dafür, dass auch alle ihr untergeordneten Filialen dieses Modell anbieten, damit die Kunden überall das neue Modell kaufen können. Nimmt hingegen eine der untergeordneten Filialen ein neues Schuhmodell in ihr Angebot auf, so hat diese Filiale keine Möglichkeit dafür zu sorgen, dass das neue Modell auch in Berlin oder in einer der anderen untergeordneten Filialen angeboten wird.

Die Filialen der zweiten Kette sind etwas anders organisiert: Die Münchner Filiale eröffnete lediglich eine untergeordnete Filiale in Nürnberg. Die Nürnberger Filiale eröffnete später selbst eine eigene untergeordnete Filiale in Frankfurt am Main. Diese Frankfurter Filiale eröffnete inzwischen auch eine untergeordnete Filiale in Stuttgart. Nimmt eine dieser Filialen einen neuen Schuh in ihr Sortiment auf, so sorgt sie immer dafür, dass auch die ihr direkt untergeordnete Filiale diesen Schuh in ihr Sortiment aufnimmt. Auf die ihr übergeordnete(n) Filiale(n) hat sie allerdings keinen Einfluss.

Insgesamt lässt sich also folgendes Muster feststellen: Eine Filiale sorgt immer und zuverlässig dafür, dass ein von ihr angebotenes neues Schuhmodell in allen der/den ihr direkt untergeordnete(n) Filiale(n) verkauft wird. Die Filiale kann hingegen nicht dafür sorgen, dass ihr übergeordnete Filialen den Schuh auch in ihr Angebot aufnehmen. “ An dieser Stelle folgte in den Instruktionen eine balancierungsabhängige Darstellung des Kausalsystems vergleichbar mit Abbildung 28.

„Nachdem die Designabteilung die vier Prototypen fertiggestellt hat, besuchst du alle Filialen persönlich, um ihnen die Modelle vorzustellen und möglichst viele Filialen dazu zu bewegen, verbindliche Vorbestellungen zu machen. Natürlich hebst Du die Vorzüge aller Prototypen gleichermaßen hervor, da du kein persönliches Interesse an der Produktion eines bestimmten Modells hast, sondern die Filialen unabhängig beraten willst. Im Anschluss an deine Präsentation der vier Prototypen entscheidet sich jede Filiale, welche der Modelle sie verbindlich vorbestellen möchte. Eine Filiale kann frei entscheiden, ob sie keines, eines oder mehrere der Modelle bestellt. Da nur ein Prototyp tatsächlich produziert werden wird, verfallen die Bestellungen für die anderen Modelle. 
Du wirst später Informationen darüber erhalten, welche der Prototypen von welchen Filialen verbindlich vorbestellt wurden. Aus diesen Informationen und dem Wissen darüber, dass die Filialen dafür sorgen, dass ein von ihnen angebotenes Modell auch in ihren Tochterfilialen angeboten wird, sollst du dann ermitteln, welches Schuhmodell sich später in den meisten Filialen vorfinden lässt. Hierbei hat es keine Bedeutung, ob ein Schuh von einer über- oder einer untergeordneten Filiale angeboten wird, es geht ausschließlich um die Anzahl der Filialen in denen er letztendlich verkauft wird. Wichtig ist außerdem, dass die Beeinflussung der untergeordneten Filiale(n) erst nach der Entscheidung für die Produktion eines bestimmten Schuhmodells stattfindet, also unabhängig von den Vorbestellungen ist. “

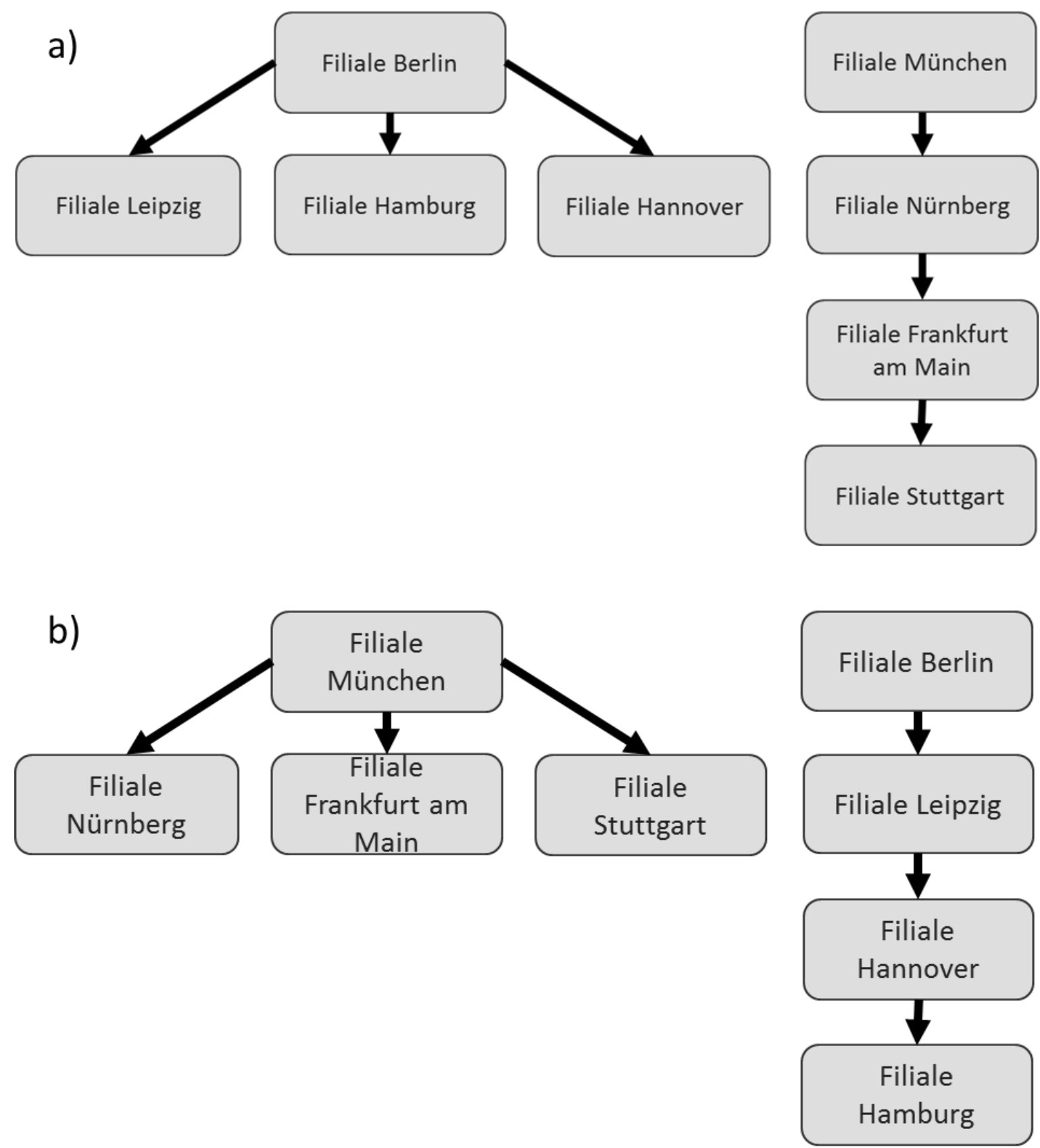

Abbildung 28: Darstellung der in Experiment 5 verwendeten Kausalsysteme in Balancierungsbedingung 1 (a) und Balancierungsbedingung 2 (b). Die Felder repräsentieren die Variablen der Systeme, die Pfeile die Kausalrelationen zwischen den Variablen, wobei die Pfeilspitze immer auf die Effekt-Variable gerichtet ist. Die Variablen können zwei verschiedene Ausprägungen besitzen: Entweder hat eine Filiale einen Schuh-Prototyp vorbestellt oder nicht. 
Die Instruktionen des kausalen Systems wurden mittels einer graphischen Darstellung visualisiert. Abbildung 28 stellt die beiden in Experiment 5 verwendeten Balancierungen dar.

\subsubsection{Multiple-Choice Wissenstest}

Der zur Abfrage des Wissens über die instruierten Kausalsysteme verwendete Multiple-Choice-Test hatte in Experiment 5 insgesamt 7 Fragen von denen 6 mit vier und eine mit drei Antwortalternativen versehen waren, von denen jeweils eine die richtige Antwort darstellte (s. Anhang H). Wie in den übrigen in dieser Arbeit vorgestellten Experimenten wurden nur die Daten von denjenigen Versuchspersonen in die Analyse aufgenommen, die alle Fragen innerhalb von 6 Durchgängen bzw. 30 Minuten korrekt beantworten konnten.

\subsubsection{Testphase}

Die Testphase von Experiment 5 wies im Vergleich zu den übrigen hier vorgestellten Experimenten die folgenden Besonderheiten auf. Die von jeder Versuchsperson zu beurteilenden vier Testfälle wurden in zufälliger Reihenfolge in einer Sequenz präsentiert, so dass insgesamt $4 * 8$ (Variablenausprägungen) $=32$ Informationen verarbeitet werden mussten. Der Ablauf der Stimuluspräsentation bestand aus der einleitenden Präsentation der später zu beantwortenden Frage für ca. 3 Sekunden. Die Frage in Experiment 5 lautete: „Wie viele Filialen werden die folgenden Schuh-Prototypen letztendlich verkaufen?“ Unmittelbar im Anschluss begann die Präsentation der Variablenausprägungen, die für die einzelnen Fälle im Block stattfand. Zunächst wurde der Name des Prototyps („Prototyp 1“, „Prototyp 2“, „Prototyp 3“, „Prototyp4“) zusammen mit der Abbildung eines Schuhes für ca. 3 Sekunden präsentiert. Danach wurde der Name des Prototyps ausgeblendet und durch die Präsentation der Variablenausprägungen ersetzt, die jeweils ca. 2,5 Sekunden auf dem Bildschirm erschienen und aus einem Satz bestanden. Die Aussage des Satzes bestand darin, ob eine Filiale den aktuellen Prototyp vorbestellt hatte oder nicht (z.B. „Hannover hat Prototyp 2 vorbestellt“; „Leipzig hat Prototyp 4 NICHT vorbestellt“). Während der Stimuluspräsentation blieb das Bild des jeweiligen Prototyps stets eingeblendet.

Im Anschluss an die Stimuluspräsentation starteten die bedingungsspezifischen Aufgaben automatisch. Unmittelbar danach (in der Bedingung spontanen Entscheidens: unmittelbar nach der Stimuluspräsentation) hatten die Versuchspersonen ihre Einschätzungen bezüglich der präsentierten Fälle abzugeben. Die Reihenfolge der vier abzugebenden Urteile war zufällig und unabhängig von der Reihenfolge in der die Fälle während der Stimuluspräsentation dargeboten wurden. Unmittelbar vor jeder Abfrage wurde ein Hinweis 
eingeblendet, der die Versuchspersonen nochmals darüber informierte, dass für die nun folgende Abgabe ihrer Einschätzung nur maximal 3 Sekunden zur Verfügung stehen. Danach wurde die bekannte elfstufige Antwortskala (von -5 bis +5) zusammen mit Abbildung und Name des zu beurteilenden Schuh-Prototyps präsentiert. Die ebenfalls eingeblendete zu beantwortende Frage lautete: „In wie vielen Filialen wird Prototyp X angeboten werden?“, wobei X der Nummer des aktuell zu beurteilenden Prototyps entsprach. Der linke (-5) Pol der Antwortskala war mit „sicher in keiner Filiale“, der rechte $(+5)$ Pol mit „sicher in allen Filialen“ gekennzeichnet. Benötigte eine Versuchsperson länger als drei Sekunden für die Abgabe einer Einschätzung wurde unmittelbar nach dem Anklicken einer Schaltfläche der Antwortskala ein Hinweisfenster eingeblendet, dass sie aufforderte, beim nächsten Mal schneller zu antworten. Unmittelbar im Anschluss wurde die Prozedur zum Abfragen der nächsten Einschätzung gestartet. Nach Abgabe aller vier Einschätzungen wurde das Experiment mit einem Bildschirm beendet, der die Versuchspersonen über den Zweck der Studie aufklärte.

Schuh 1
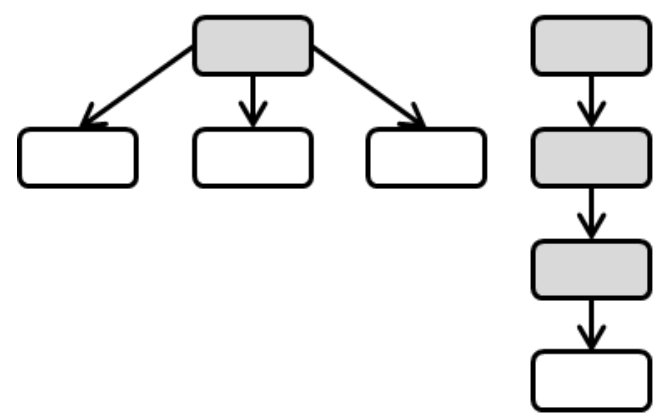

Schuh 3
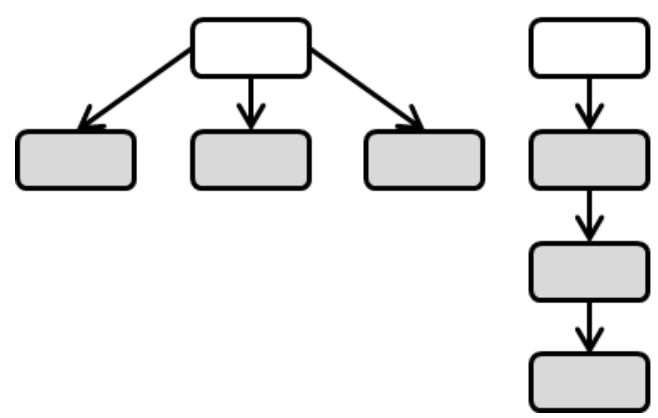

Schuh 2
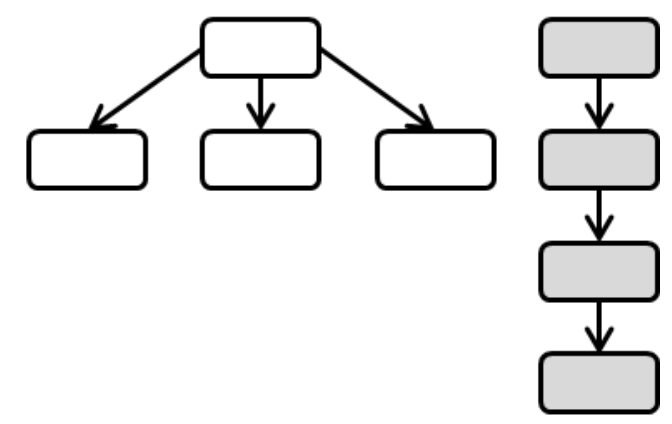

Schuh 4
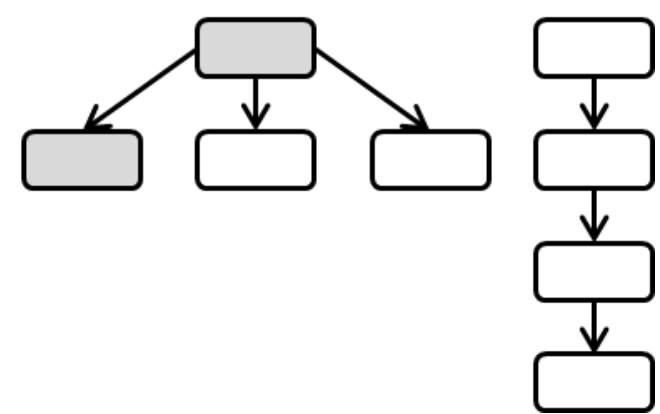

Abbildung 29: Schematische Darstellung der in Experiment 5 verwendeten Fälle. Grau gefärbte Felder repräsentieren Vorbestellungen für den jeweiligen Schuh-Prototyp in der entsprechenden Filiale.

Die in Experiment 5 präsentierten Fälle sind in Abbildung 29 graphisch dargestellt. Das letztendliche Angebot, das die Versuchspersonen für eine richtige Beurteilung der vier Prototypen vorherzusagen hatten ist in Abbildung 30 dargestellt. Aus Abbildung 30 geht 
hervor, dass für Schuh 1 die höchsten Ratings zu erwarten sind. Die zweithöchsten Ratings sollte Schuh 3 auf sich ziehen, während die Schuhe 2 und 4 mit den niedrigsten Ratings verbunden sein sollten.

Schuh 1
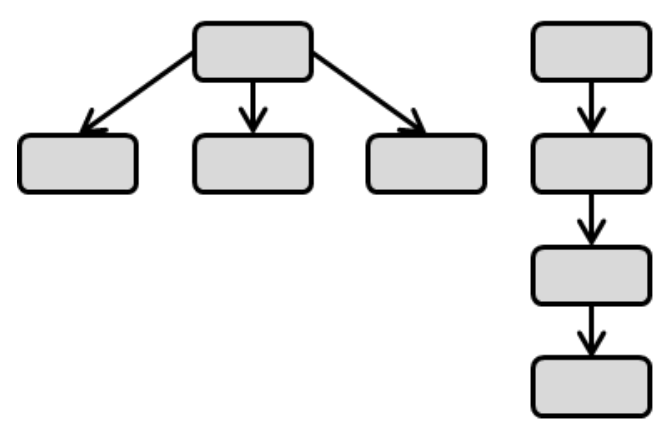

Schuh 3
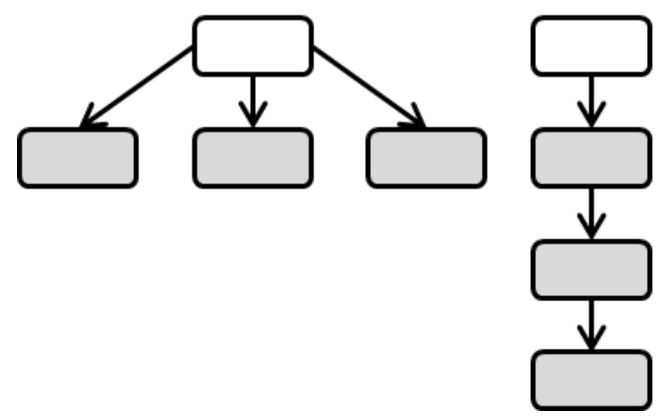

Schuh 2
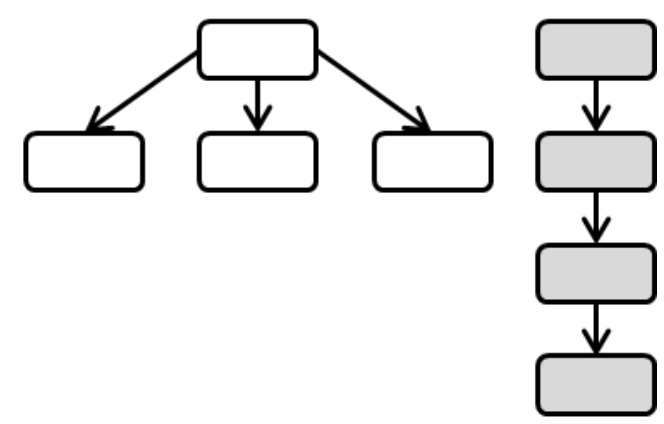

Schuh 4
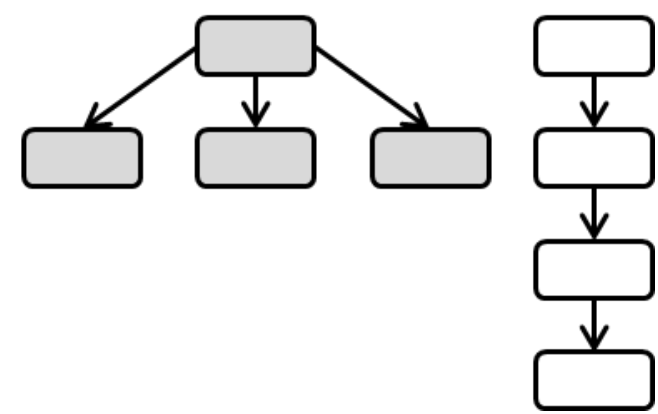

Abbildung 30: Schematische Darstellung des letztendlichen Angebotes für die vier Schuh-Prototypen in Experiment 5. Grau gefärbte Felder repräsentieren die Geschäfte, in denen der jeweilige Schuh-Prototyp nach Wirken der kausalen Mechanismen angeboten werden würde.

\subsubsection{Ergebnisse}

15 der 101 Versuchspersonen konnten nicht alle Fragen des Multiple-Choice Wissenstests innerhalb von 6 Durchgängen bzw. 30 Minuten korrekt beantworten. Somit können die Daten von 86 Versuchspersonen für die weiteren Analysen verwendet werden. Von diesen befanden sich 22 in der Bedingung spontanen Entscheidens, 22 in der Bedingung unbewussten Denkens, 22 in der Bedingung bewussten Denkens mit festgelegter Dauer und 20 in der Bedingung bewussten Denkens mit selbstgewählter Dauer. 62 der 86 Versuchspersonen, von denen Daten in der folgenden Analyse verwendet wurden waren weiblichen Geschlechts (72\%). Psychologiestudierende bildeten mit 56 Personen (65\%) die mit Abstand größte Gruppe in der aus Studenten der Universität Göttingen bestehenden Stichprobe. Die 86 Versuchspersonen benötigten durchschnittlich 18 Minuten für die Durchführung des Experiments (spontanes Entscheiden: 15 Minuten; unbewusstes Denken: 20 Minuten; bewusstes Denken mit festgelegter Dauer: 20 Minuten; bewusstes Denken mit 
selbstgewählter Dauer: 16 Minuten). Aufgrund eines Fehlers bei der Datenaufzeichnung konnte die durchschnittliche Dauer des Überlegens in der Bedingung bewussten Denkens mit selbstgewählter Dauer nicht aufgezeichnet werden.

Für die nachfolgende Analyse wurden zunächst die Ratings für die Fälle 2 und 4 auf individueller Ebene gemittelt, da sie die gleiche Anzahl an zu erwartenden Effekten hatten. Es stellte sich jedoch heraus, dass die resultierenden Fall-Cluster eine so große Anzahl an fehlenden Werten besaßen, dass eine Erfassung der allgemeinen Antwortmuster mittels Varianzanalyse nicht sinnvoll war. Um die Anzahl der fehlenden Werte auf ein erträgliches Maß zu reduzieren wurden daher die Fälle 2, 3 und 4 auf individueller Ebene gemittelt, um sie später mit Fall 1, von dem das höchste Rating erwartet wurde, zu kontrastieren. Diese Methode des Vergleichs der Antworten für den Fall mit dem höchsten erwarteten Urteil mit den gemittelten Antworten der restlichen Fälle wurde bereits für Untersuchungen im Rahmen der Unconscious Thought Theory (z.B. Waroquier et al., 2010) genutzt und kann daher auch für die vorliegende Analyse als adäquat angesehen werden.

\subsubsection{Multiple-Choice Wissenstest}

In Abbildung 31 ist die Anzahl der durchschnittlich benötigten Lerndurchgänge $(1,67)$ in den verschiedenen Verarbeitungsbedingungen dargestellt. Es ergaben sich keine Unterschiede zwischen den verschiedenen Verarbeitungsbedingungen $(F(3,82)<1, p=0,52$, $M S E<1)$. Die Graphik basiert auf denjenigen 86 Versuchspersonen, die letztlich alle MultipleChoice-Fragen innerhalb von 6 Durchgängen korrekt beantworten konnten. Von den 15 Versuchspersonen (15\%), die nicht alle Fragen korrekt beantworten konnten entfielen 4 auf die Bedingung sofortigen Entscheidens, 5 auf die Bedingung unbewussten Denkens, 4 auf die Bedingung bewussten Denkens mit festgelegter Dauer und 2 auf die Bedingung bewussten Denkens mit selbstgewählter Dauer. 


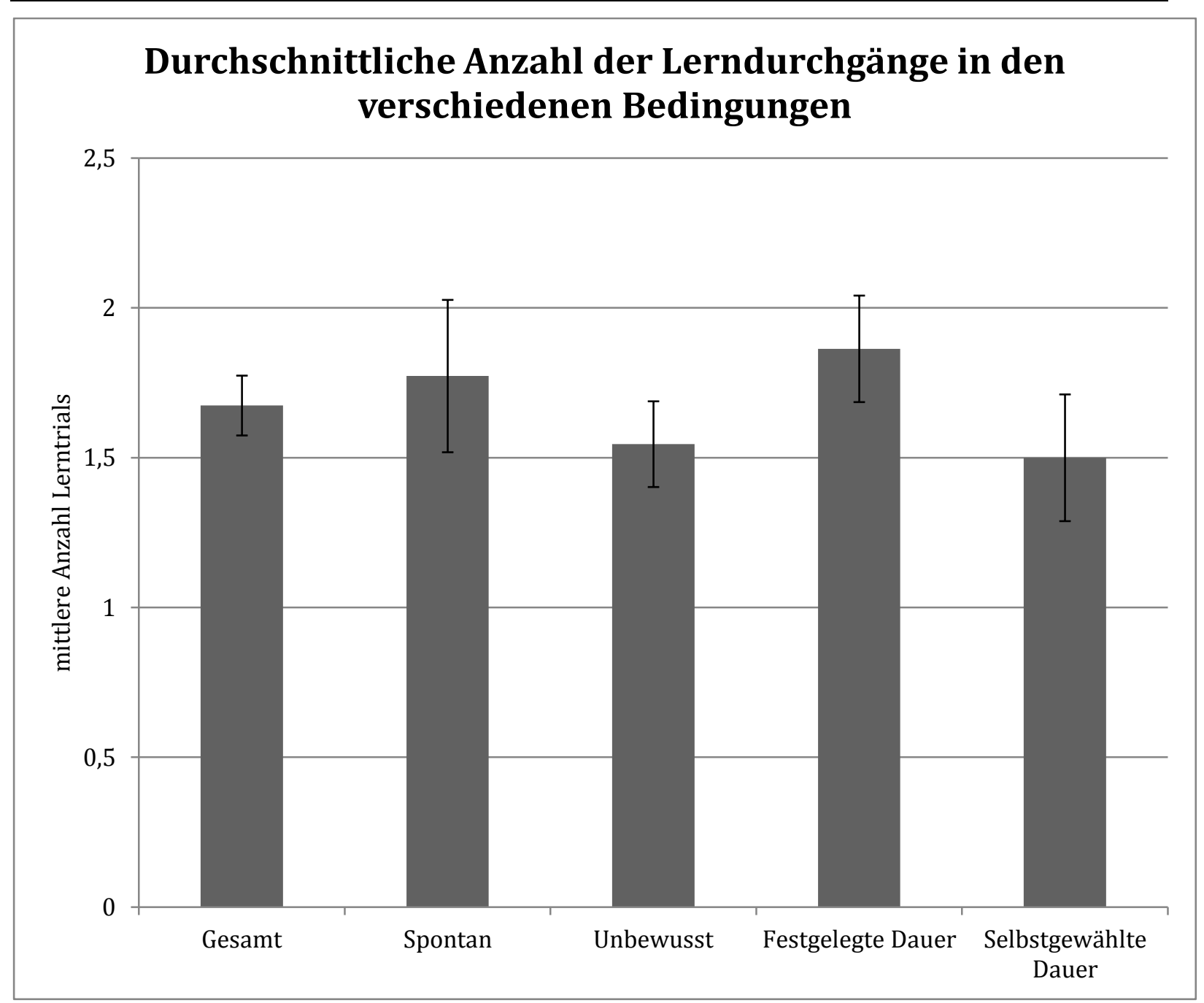

Abbildung 31: Durchschnittliche Anzahl der benötigten Lerndurchgänge (und deren Standardfehler) in den verschiedenen Verarbeitungsbedingungen von Experiment 5.

\subsubsection{Vorhersagen auf Grundlage kausaler Mechanismen}

Zunächst wurden die Antwortmuster der Urteile, die innerhalb von 3 Sekunden nach dem Erscheinen des Antwortfensters abgegeben wurden mit denjenigen verglichen, die innerhalb von 5 Sekunden abgegeben wurden. Die hierfür verwendeten zweifaktoriellen Varianzanalysen mit Messwiederholungen (Gruppierungsfaktor: Verarbeitungsbedingungen; Messwiederholungsfaktor: Fall-Cluster) ergaben jeweils einen signifikanten Effekt der FallCluster (3 Sekunden: $F(1,37)=5,68, p<0,05, M S E=1642 ; 5$ Sekunden: $(F(1,63)=10,98$, $p<0,01, M S E=2941$ ), keinen Effekt für die Manipulation der Verarbeitungsbedingung (3 Sekunden: $F(3,37)<1, p=0,70, M S E=201$; 5 Sekunden: $(F(3,63)<1, p=0,59, M S E=180)$ und keine signifikante Interaktion zwischen Verarbeitungsbedingungen und Fall-Clustern (3 Sekunden: $F(3,37)<1, p=0,71, M S E=134 ; 5$ Sekunden: $(F(3,63)<1, p<0,93, M S E=41)$. Wurden für die innerhalb von 5 Sekunden abgegebenen Urteile die fehlenden Werte durch den Durchschnitt für das jeweilige Fall-Cluster über alle Bedingungen ersetzt, so ergab sich 
hierdurch ebenfalls keine wesentliche Veränderung im Antwortmuster (Fall-Cluster: $F(1,82)=17,87, p<0,001, M S E=4135$; Verarbeitungsbedingung: $F(3,82)<1, p=0,67, M S E=127$; Interaktion zwischen Verarbeitungsbedingungen und Fall-Clustern: $F(3,82)<1, p=0,87$, $M S E=54)$. Somit werden für die folgenden Kontrastanalysen die Antworten herangezogen, die innerhalb von 5 Sekunden abgegeben wurden, und bei denen die fehlenden Werte ersetzt sind. Eine graphische Darstellung der Ratings für die beiden Fall-Cluster in den verschiedenen Verarbeitungsbedingungen findet sich in Abbildung 32.

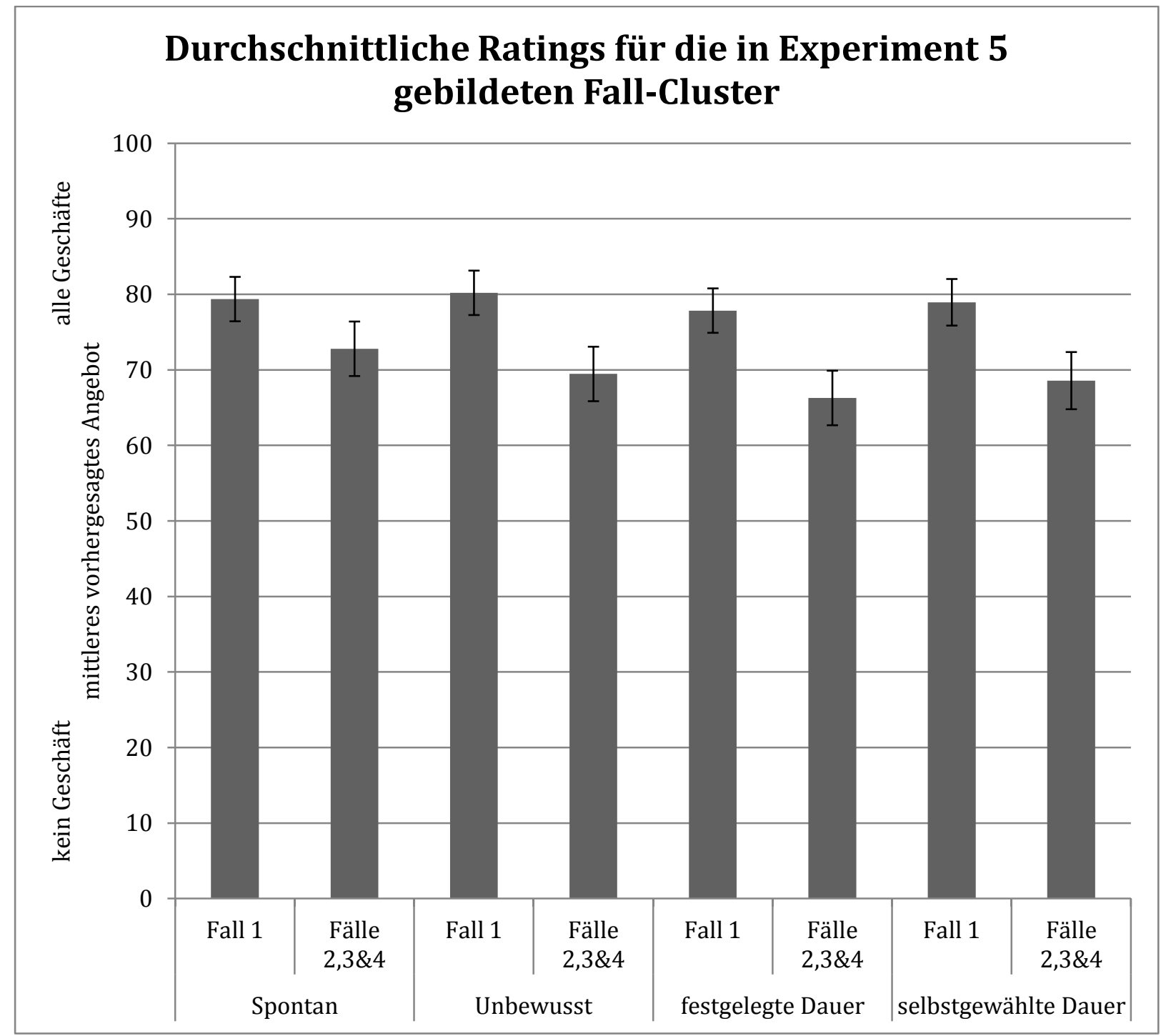

Abbildung 32: Durchschnittliche Ratings (und Standardfehler) bezüglich des vorhergesagten Angebots für die beiden Fall-Cluster in den verschiedenen Verarbeitungsbedingungen von Experiment 5.

Die Analyse der Urteile für die beiden Fall-Clustern ergab zunächst signifikante Unterschiede über alle Bedingungen $\left(t_{(85)}=4,23, p<0,001\right)$. Eine Betrachtung für die verschiedenen Verarbeitungsbedingungen zeigte, dass dieser Effekt auf signifikanten Unterschieden in den Bedingungen unbewussten Denkens $\left(t_{(21)}=2,34, p<0,05\right)$, bewussten 
Denkens mit festgelegter Dauer $\left(t_{(21)}=2,52, \quad p<0,05\right)$ und bewussten Denkens mit selbstgewählter Dauer $\left(t_{(19)}=2,16, p<0,05\right)$ beruht. Versuchspersonen in der Bedingung spontanen Entscheidens differenzierten hingegen nicht zwischen den beiden Fall-Clustern $\left(t_{(21)}=1,43, \quad p=0,16\right)$. Keiner dieser Unterschiede war jedoch groß genug, um eine Überlegenheit einzelner Verarbeitungsbedingungen gegenüber anderen zu bewirken (alle $p$ 's zwischen den Bedingungen > 0,44, s. Anhang I).

\subsubsection{Diskussion}

Die Ergebnisse von Experiment 5 zeigen, dass lediglich diejenigen Versuchspersonen, die ihr Urteil direkt im Anschluss an die Präsentation der Stimuli abzugeben hatten nicht in der Lage waren, zwischen Fall-Clustern mit unterschiedlichem erwartetem Urteil zu differenzieren. Die Überlegenheit der Differenzierungsleistung der übrigen Verarbeitungsbedingungen erwies sich jedoch nicht als signifikant. Entgegen den Vorhersagen der Unconscious Thought Theory (Dijksterhuis \& Nordgren, 2006) konnte auch keine Überlegenheit von Entscheidungen nach einer Phase des unbewussten Denkens nachgewiesen werden, auch wenn die Versuchspersonen in dieser Bedingung in der Lage waren zwischen Fall-Clustern mit unterschiedlichem erwarteten Rating zu differenzieren.

Die Ergebnisse der Bedingungen bewussten Denkens zeigen, dass die Versuchspersonen hier ebenfalls zwischen den Fall-Clustern mit unterschiedlichem erwarteten Rating differenzieren konnten. Da jedoch keine Überlegenheit der Bedingungen bewussten Nachdenkens gegenüber der Bedingung spontanen Entscheidens festgestellt werden konnte und zudem auch die Versuchspersonen in der Bedingung unbewussten Denkens erfolgreich differenzierten, konnten insgesamt keine Belege für die Notwendigkeit von Typ 2-Verarbeitungsprozessen für die erfolgreiche Bewältigung der in Experiment 5 getesteten, mentale Simulationen erfordernde, Aufgaben gesammelt werden.

Eine Auffälligkeit in den Ergebnissen ist, dass sich in allen Verarbeitungsbedingungen nur geringe Unterschiede in den Urteilen der Versuchspersonen für die beiden Fall-Cluster zeigen. Ein Grund hierfür könnte in der Beschriftung der 11-stufigen Antwortskala gelegen haben, die bei korrekter Berücksichtigung der kausalen Mechanismen nur Antworten zwischen 0 und +5 ermöglichte und dadurch die Spannweite der Ratings eingeschränkt haben könnte. Diese Annahme wird durch das Antwortverhalten der Versuchspersonen gestützt: Nur 31 der 280 (11\%) über alle Bedingungen innerhalb von 5 Sekunden nach Erscheinen der Antwortskala abgegebenen Urteile lagen im Bereich von -5 bis -1 . 


\section{Allgemeine Diskussion}

Im nun folgenden Abschnitt soll zunächst die Beurteilung der aufgestellten Hypothesen im Lichte der Ergebnisse der durchgeführten Experimente diskutiert werden. Dazu sollen die Ergebnisse der durchgeführten Experimente zusammenhängend dargestellt und mögliche Gründe für die vorgefundenen Ergebnismuster diskutiert werden. In diesem Zusammenhang werden auch Möglichkeiten aufgezeigt, wie die angebotenen Erklärungen empirisch überprüft werden könnten. Im Anschluss an die hypothesengeleitete Bewertung der Ergebnisse soll die mögliche Rolle des unbewussten Denkens im Bereich des kausalen Urteilens und Entscheidens diskutiert werden.

\subsection{Bewertung von Hypothese 1 anhand der Ergebnisse der Kategorisierungsexperimente}

Mit den in den Experimenten 1, 2 und 3 verwendeten Kategorisierungsaufgaben sollte getestet werden, ob Versuchspersonen auch auf der Grundlage von Typ 1Verarbeitungsprozessen kausale Faktoren bei ihren Urteilen berücksichtigen. Dabei hatten sie zuvor erlerntes Wissen über ein fiktives Kausalsystem mit Informationen über die Variablenausprägungen einer Instanz dieses Systems zu kombinieren und anschließend eine Bewertung abzugeben. Diese Bewertung betraf die Zugehörigkeit der präsentierten Instanz zu einer durch das jeweilige Kausalsystem beschriebenen Kategorie. Zwei Fragen standen bei der Beurteilung der Ergebnisse im Mittelpunkt: Die erste Frage ist die nach Belegen für überzufällig gute Kategorisierungsleistungen auf Basis von Typ 1-Verarbeitungsprozessen. Hierzu wurden die Urteile der Versuchspersonen für die verschiedenen präsentierten Fälle in den Bedingungen spontanen Entscheidens analysiert. Die zu beurteilenden Fälle waren so konstruiert, dass für sie auf Grundlage des instruierten Kausalsystems unterschiedliche Kategorisierungsurteile erwartet werden konnten. Eine überzufällig gute Kategorisierungsleistung lag vor, wenn signifikant unterschiedliche Ratings für Fälle mit unterschiedlichen erwarteten Kategorisierungsurteilen abgegeben wurden. Zweitens sollte geprüft werden, ob Versuchspersonen, die nach der Stimuluspräsentation die Möglichkeit erhielten, ihr Urteil mittels bewusster Typ 2-Verarbeitungsprozessen zu bilden, zu besseren Urteilen (im Sinne einer Berücksichtigung kausaler Faktoren wie kausalem Status oder kausaler Kohärenz) gelangen. Mit Hypothese 1 wurde vorhergesagt, dass dies nicht der Fall sein würde. Zur Überprüfung dieser Fragestellung wurde getestet, ob die Differenzierungsleistungen in den Bedingungen, in denen bewusst (Typ 2-) Verarbeitungsprozesse möglich waren, signifikant besser waren als die 
Differenzierungsleistung in der jeweiligen Bedingung spontanen Entscheidens. Die Bedingungen, in denen Typ 2-Verarbeitungsprozesse möglich sein sollten waren die Bedingung bewussten Nachdenkens mit festgelegter Dauer in Experiment 1, die Bedingung bewussten Nachdenkens mit selbstgewählter Dauer in den Experimenten 2 und 3, sowie die Bedingung der Online-Verarbeitung in Experiment 3.

Die Ergebnisse der drei Experimente, in denen Kategorisierungsaufgaben getestet wurden ergaben ein uneinheitliches Bild hinsichtlich des Vermögens, mittels Typ 1-Prozessen zwischen Fällen mit unterschiedlichem erwarteten Kategorisierungsurteil zu unterscheiden. Die Versuchspersonen in der Bedingung spontanen Entscheidens in Experiment 1 differenzierten offenbar nicht auf der Grundlage des instruierten Kausalsystems sowie der angenommenen kausalen Faktoren. Daher können die Ergebnisse dieses Experiments nicht für die Untersuchung von Hypothese 1 herangezogen werden. Die Analyse der Urteile in der Bedingung spontanen Entscheidens von Experiment 2 ergab keine signifikanten Unterschiede zwischen den Fall-Clustern. Die Versuchspersonen in der Bedingung spontanen Entscheidens von Experiment 3 waren hingegen in der Lage, zwischen Fällen mit unterschiedlichen erwarteten Kategorisierungsurteilen zu differenzieren. Allerdings konnte entsprechend der Vorhersage von Hypothese 1 in keinem der drei Experimente eine signifikant bessere Differenzierungsleistung in denjenigen Bedingungen festgestellt werden, die Typ 2Verarbeitungsprozesse ermöglichten. Im Folgenden soll diskutiert werden, warum die Differenzierungsleistungen in den Bedingungen spontanen Entscheidens uneinheitlich waren. Hierbei wird auf die Besonderheiten der einzelnen Experimente eingegangen und eine spekulative Erklärung für die unterschiedlichen Befunde angeboten.

Eine bereits in der Vorstellung von Experiment 1 erwähnte Erklärung für die gefundenen Ergebnisse besteht darin, dass die Versuchspersonen durch einfaches Abzählen der für eine Kategorienzugehörigkeit charakteristischen Variablenausprägungen während der Stimuluspräsentation (im Folgenden: Online-Tallying) zu einem Urteil gelangen konnten. Da ein Urteil auf diese Weise unabhängig vom zuvor erlernten Kausalwissen zustande kommen kann, sind die Ergebnisse der Kategorisierungsbedingungen von Experiment 1 ungeeignet, um Aussagen über die Differenzierungsleistung auf Grundlage kausalen Wissens mittels Typ 1-Verarbeitungsprozessen $\mathrm{zu}$ treffen. Die Konsequenz aus dieser Erklärung für die Experimente 2 und 3 bestand in der Konstruktion von Testfällen, die eine Anwendung des erlernten Kausalwissens erforderten, um zwischen den Fällen differenzieren zu können. Dies geschah, indem die Testfälle in den beiden Experimenten jeweils gleich viele für eine 
Kategorienzugehörigkeit charakteristische Variablenausprägungen aufwiesen und nur über deren kausalen Status unterschieden werden konnten. Die Fälle waren darüber hinaus so gestaltet, dass diejenigen mit auf Grundlage des kausalen Status hohen erwarteten Kategorisierungsurteilen zugleich eine hohe kausale Kohärenz aufwiesen und ein niedriges erwartetes Kategorisierungsurteil auf Grundlage des kausalen Status mit einer niedrigeren kausalen Kohärenz einherging. Die Strategie des Online-Tallying ist somit in diesen Experimenten ungeeignet, um zwischen den Fällen mit unterschiedlichem erwarteten Kategorisierungsurteil zu differenzieren.

Die nicht vorhandene Differenzierungsleistung der Versuchspersonen in der Bedingung spontanen Entscheidens von Experiment 2 kann in diesem Sinne als ein Indiz für das Vorliegen von Online-Tallying als Entscheidungsstrategie und gegen kausalmodellbasierte Kategorisierung gesehen werden. Allerdings erbrachten die Versuchspersonen in der Bedingung spontanen Entscheidens von Experiment 3 signifikante Differenzierungsleistungen, obwohl Online-Tallying hier ebenfalls nicht geeignet war zwischen Fällen mit unterschiedlichem erwarteten Kategorisierungsurteil zu differenzieren. Es stellt sich somit die Frage nach Unterschieden zwischen Experiment 2 und Experiment 3, die dazu geführt haben könnten, dass Online-Tallying in Experiment 2 als Entscheidungsstrategie angewandt wurde, während die Urteile in Experiment 3 offensichtlich auf Grundlage des instruierten Kausalmodells getroffen wurden.

Die Unterschiede zwischen den beiden Experimenten betreffen die verwendete Kausale Struktur, die benutzte Coverstory, die präsentierten Testfälle sowie eine leicht veränderte Aufgabenstellung. Die Unterschiede in der kausalen Struktur bestehen in der Anzahl der Variablen (10 in Experiment 2, 13 in Experiment 3), der Anzahl der kausalen Beziehungen (10 in Experiment 2, 12 in Experiment 3), sowie der Existenz zweier Gemeinsame-Ursache Variablen in Experiment 2. Zudem gab es in Experiment 2 zwei Variablen, die gemeinsam den höchsten kausalen Status aufwiesen, da sie nicht von anderen Variablen des Systems beeinflusst wurden. Hingegen gab es in Experiment 3 nur eine Variable, die nicht von anderen Variablen abhing und dadurch auch den höchsten kausalen Status aufwies. Insgesamt kann jedoch festgehalten werden, dass sich die kausalen Strukturen in beiden Experimenten relativ ähnlich sind (vgl. die Abbildungen 12 und 18 für die kausalen Strukturen und die verwendeten Testfälle in beiden Experimenten). So weist die Struktur Experiment 3 zwar je zwei zusätzliche Variablen und Kausalstrukturen auf, ist aber dafür durch die fehlenden Gemeinsamer-Effekt-Variablen etwas einfacher aufgebaut. Es ist daher 
kein offensichtlicher auf die kausale Struktur der Experimente zurückzuführender Grund für die unterschiedlichen Leistungen in den beiden Experimenten zu erkennen.

Ein weiterer Unterschied zwischen Experiment 2 und Experiment 3 ist die zur Vermittlung des Kausalsystems verwendete Coverstory. Während den Versuchspersonen in Experiment 2 eine Warane betreffende Krankheit instruiert wird, handelt es sich in Experiment 3 um ein neues psychische Störungsmodell. Bei der Erstellung der Coverstories wurde in beiden Fällen darauf geachtet, dass die einzelnen Kausalbeziehungen plausibel sind (so ist es z.B. in der Coverstory zu Experiment 2 sinnvoll, anzunehmen, dass Erbrechen zu einer Schädigung der Speiseröhre führen kann). Da jedoch Studierende der Psychologie die größte Subgruppe unter den Versuchspersonen ausmachten (ihr Anteil lag bei $45 \%$ in Experiment 2 bzw. 40\% in Experiment 3) könnte man annehmen, dass die Coverstory von Experiment 3 für diese Versuchspersonen eingängiger ist, da sie ihren Interessen und Erfahrungen näher steht. Eine Betrachtung der durchschnittlich benötigten Lerntrials für Psychologiestudierende und nicht-Psychologiestudierende in Experiment 3 ergab allerdings keine signifikanten Unterschiede $\left(t_{(83)}=-063, p=0,53\right)$ zwischen den beiden Gruppen. Dieses Ergebnis spricht gegen den Erklärungsansatz einer intuitiv zugänglicheren Coverstory in Experiment 3. Des Weiteren unterschieden sich die beiden Experimente hinsichtlich der Unterscheidbarkeit der Variablen mit dem höchsten kausalen Status in den jeweiligen Kausalsystemen. In Experiment 2 wurden diese als „Adenobakterien, bzw. „Coronabakterien“ präsentiert. Hier könnte es aufgrund der Ähnlichkeit der Variablennamen $\mathrm{zu}$ Verwechslungen während der Stimuluspräsentation gekommen sein, die die Versuchspersonen verwirrt haben könnten. Diese Gefahr bestand bei Experiment 3 nicht, da die Variablen mit dem höchsten kausalen Status hier als „Gefühlter Kontrollverlust“ und „Starke Stressreaktion“ benannt wurden und somit gut unterscheidbar waren. Gegen den Einfluss der Variablenbezeichnungen spricht jedoch die Konstruktion der präsentierten Testfälle: In beiden Experimenten waren alle Fälle (mit Ausnahme des für die Differenzierungsleistung unkritischen Falles 5 in Experiment 3, bei dem alle charakteristischen Variablenausprägungen der PED vorhanden waren) so konstruiert, dass die beiden präsentierten Variablen mit dem höchsten kausalen Status jeweils die gleiche Ausprägung hatten.

Eine Spekulation über Unterschiede zwischen den beiden Experimenten, die zu den beobachteten Ergebnissen geführt haben könnte, bezieht sich auf eine Modifikation der Art des von den Versuchspersonen abzugebenden Urteils. In Experiment 2 sollten sie bei der 
Beurteilung der präsentierten Fälle entscheiden, ob der jeweilige Waran an der Torokrankheit oder einer anderen Krankheit leidet. Sie hatten also die präsentierten Variablenausprägungen im Hinblick auf zwei verschiedene Kategorien vergleichend zu evaluieren. Es ist möglich, dass hierbei ein zweistufiger Entscheidungsprozess zur Anwendung kommt: In einem ersten Schritt werden die Variablenausprägungen hinsichtlich der Passung in das instruierte Kausalsystem bewertet. In einem zweiten Schritt wird bewertet, ob diese Passung ausreicht, um vom Vorliegen einer Torokrankheit auszugehen, oder ob es wahrscheinlicher ist, dass die präsentierten Symptome von einer anderen Krankheit verursacht worden sind. Da den Versuchspersonen in der Bedingung spontanen Entscheidens klar war, dass sie unmittelbar nach der Stimuluspräsentation ein Urteil abzugeben hatten, könnten sie versucht haben, bereits online $\mathrm{zu}$ einem Urteil zu gelangen. Hierfür wäre der geschilderte zweistufige Entscheidungsprozess aber zu komplex, da die Ausführung des zweiten Schrittes erst nach der Stimuluspräsentation erfolgen kann. Als alternative Entscheidungsstrategie bietet sich mit dem Online-Tallying eine Möglichkeit an, die zu treffende Wahl zwischen zwei Alternativen (Torokrankheit vs. andere Krankheit) mit überschaubarem Aufwand bereits während der Stimuluspräsentation bewerkstelligen zu können. Die Wahl dieser Strategie führte in Experiment 1, in dem sich die Versuchspersonen ebenfalls zwischen zwei Alternativen (echter Schamane vs. falscher Schamane) entscheiden mussten aufgrund der bereits dargestellten Fallgestaltung zu unterschiedlichen Beurteilungen der präsentierten Fälle. In Experiment 2, für das die Fälle ja speziell so konstruiert wurden, dass eine Differenzierung mit Online-Tallying nicht mehr möglich war, führte sie hingegen zu den beobachteten fehlenden Unterschieden in den Urteilen der Probanden.

Die Art der zu bearbeitenden Aufgabe unterschied sich in Experiment 3 dadurch, dass hier gefragt wurde, mit welcher Wahrscheinlichkeit der präsentierte Patient an einer PostEmbitterment-Disorder leidet. Der Fall war somit lediglich im Hinblick auf die Passung zu der instruierten Kategorie zu beurteilen. Dies entspricht dem ersten Schritt des angenommenen zweistufigen Entscheidungsprozesses in Experiment 2. Das abzugebende Urteil konnte ohne weitere vorzunehmende Bewertungen direkt aus dem Ergebnis dieser ersten Stufe abgeleitet werden. Somit ist die Entscheidungsaufgabe in Experiment 3 weniger komplex als in Experiment 2 und es kann angenommen werden, dass die erforderlichen Bewertungsprozesse während der Stimuluspräsentation durchgeführt werden können. Auf diese Weise könnten die guten Differenzierungsleistungen der Versuchspersonen in der Bedingung spontanen Entscheidens in Experiment 3 erklärt werden, da die Urteile tatsächlich auf der Grundlage des instruierten Kausalsystems getroffen wurden. Zudem ist davon 
auszugehen, dass die Urteile aufgrund der zeitlichen Restriktionen während der Stimuluspräsentation auf Typ 1-Verarbeitungsprozessen basieren.

Die hier vorgestellten Experimente hatten nicht zum Ziel, Unterschiede zwischen dem Einfluss von kausalem Status und kausaler Kohärenz wie beispielsweise bei Rehder (2010) zu untersuchen. Dennoch wäre es mit dem im Rahmen dieser Arbeit vorgestellten experimentellen Paradigma möglich $\mathrm{zu}$ untersuchen, ob sich spontan abzugebende Kategorisierungsurteile für Fälle, für die nach kausalem Status und kausaler Kohärenz unterschiedliche Vorhersagen gemacht werden, eher am kausalen Status der Variablen oder der kausalen Kohärenz orientieren. Darüber hinaus könnte untersucht werden, ob sich spontan abgegebene Urteile von solchen unterscheiden, bei denen bewusst über die Antwort nachgedacht werden kann. Auf diese Weise könnte ein Einblick in die intuitive bzw. deliberative Verwendung von Status- bzw. Kohärenzinformationen gewonnen werden.

Die Spekulation über die Entscheidungsprozesse in den Bedingungen spontanen Entscheidens wirft natürlich die Frage nach den Vorgängen in den anderen Verarbeitungsbedingungen auf, in denen die Versuchspersonen nach der Stimuluspräsentation noch Zeit hatten, bevor sie ihr Urteil abgeben mussten. Die Bedingungen der Stimuluspräsentation waren in diesen Verarbeitungsbedingungen identisch mit denen in der Bedingung des spontanen Entscheidens. Daher liegt die Vermutung nahe, dass die Versuchspersonen auch hier in der Lage waren, die präsentierten Informationen aufzunehmen und ein auf Typ 1-Verarbeitungsprozessen basierendes Urteil zu bilden. Im Anschluss hatten sie jedoch die Gelegenheit, dieses Urteil zu evaluieren und gegebenenfalls zu korrigieren. Dies konnte in der Bedingung bewussten Denkens auf der Grundlage von Typ 2-Prozessen geschehen. In der Bedingung unbewussten Denkens bestand hingegen die Möglichkeit des Wirkens unbewusster, langsamer Verarbeitungsprozesse, die den Vertretern der Unconscious Thought Theory (Dijksterhuis \& Nordgren, 2006) zufolge ebenfalls in der Lage sind, bestehende Repräsentationen im Hinblick auf eine zu lösende Aufgabe zu modifizieren (Strick, Dijksterhuis \& van Baaren, 2010). Eine solche bewusst oder unbewusst durchgeführte Re-Evaluation eines Online gebildeten Urteils könnte erklären, warum die Versuchspersonen in den Bedingungen in Experiment 2, in denen sie nicht spontan entscheiden mussten in der Lage waren, zwischen den Fällen mit unterschiedlichem erwarteten Kategorisierungsurteil zu differenzieren (es ergaben sich signifikante Unterschiede in der Bedingung bewussten Nachdenkens mit selbstgewählter Dauer und marginal signifikante Unterschiede in der Bedingung unbewussten Denkens). Die Ergebnisse von Experiment 3 sind ebenfalls 
konsistent zu dieser Erklärung: Hier war das während der Stimuluspräsentation gebildete Urteil aus den oben genannten Gründen bereits korrekt und musste daher nicht mehr nachträglich korrigiert werden (vgl. Abschnitt 3.1).

Die von den Versuchspersonen frei wählbare Dauer der Stimuluspräsentation in der Bedingung der Online-Verarbeitung in Experiment 3 ist der Grund, warum die obige Erklärung nicht auf diese Bedingung angewendet wird. Da die Versuchspersonen in dieser Bedingung sowohl von der zeitlichen Einschränkung bzgl. der Abgabe ihrer Urteile als auch von der zeitlich nicht eingeschränkten Stimuluspräsentation wussten, hatten sie die Möglichkeit, bereits während der Präsentation mittels Typ 2-Verarbeitungsprozessen zu einem Urteil zu gelangen. Diese Vermutung steht in einem scheinbaren Widerspruch zur Annahme von auf Typ 1-Prozessen basierenden Urteilen, die unmittelbar nach der Stimuluspräsentation abgegeben werden (vgl. Mamede et al., 2010; Thompson et al., 2011). Ein wesentlicher Unterschied zu den Studien von Mamede et al. (2010), sowie Thompson et al. (2011), bei denen die Stimuluspräsentation ebenfalls ohne zeitliche Restriktionen erfolgte, besteht im Wissen um die Erfordernis einer schnellen Urteilsabgabe in den in dieser Arbeit berichteten Experimenten. Werden die Versuchspersonen nicht darüber informiert, dass ihnen nach der Stimuluspräsentation nur eine begrenzte Zeitspanne zur Abgabe ihres Urteils zur Verfügung steht, so haben sie auch keinen Grund, eventuell zusätzlich zur Verfügung stehende Zeit während der Präsentation zum bewussten Lösen der Aufgabe bereits während der Präsentation zu nutzen.

Bei Experimenten ohne zeitliche Restriktionen bezüglich der Dauer der Stimuluspräsentation und des für die Abgabe eines Urteils zur Verfügung stehenden Zeitraums (z.B. Mamede et al., 2010, Thompson et al., 2011) kann nicht zwangsläufig davon ausgegangen werden, dass keine zusätzlichen mit der Lösung der Aufgabe befassten Typ 2Prozesse ablaufen. Um aufgabenbezogene Typ 2-Verarbeitungsprozesse während der Informationsaufnahme zu verhindern, wurde daher in den in dieser Arbeit vorgestellten Experimenten die Dauer der Stimuluspräsentation in allen Bedingungen (mit Ausnahme der Bedingung der Online-Verarbeitung) begrenzt. Die Aufhebung dieser Begrenzung in Kombination mit dem Wissen um die Erfordernis einer schnellen Urteilsabgabe in der Bedingung der Online-Verarbeitung sollte hingegen auf die Lösung der Aufgabe ausgerichtete Typ 2-Verarbeitungsprozesse bereits während der Stimuluspräsentation ermöglichen. Letztlich kann aufgrund der vorhergesagten und festgestellten Gleichartigkeit der Ergebnisse in der Bedingung spontanen Entscheidens und der Bedingung der Online- 
Verarbeitung jedoch nicht über die Existenz von aufgabenbezogenen Typ 2Verarbeitungsprozessen während der Stimuluspräsentation in der letztgenannten Bedingung entschieden werden. Aufgrund der hier dargestellten Argumentation erscheint diese jedoch wahrscheinlich. Für die Untersuchung der Annahme, dass Versuchspersonen in der Bedingung der Online-Verarbeitung von Experiment 3 bereits während der Stimuluspräsentation mittels Typ 2-Prozessen an der Lösung der Aufgabe arbeiteten wäre ein Experiment denkbar, bei dem Typ 2-Verarbeitungsprozesse zur erfolgreichen Bewältigung einer Aufgabe erforderlich sind. Ergäben sich in der Bedingung der Online-Verarbeitung bessere Ergebnisse als in der Bedingung spontanen Entscheidens, so könnte dies als Beleg für die Gültigkeit der vorgestellten Argumentation gewertet werden.

Insgesamt können die Ergebnisse der vorgestellten Experimente die für die Verarbeitungsprozesse bei Kategorisierungen aufgestellte Hypothese 1 mit Einschränkungen bestätigen. Diese Einschränkungen betreffen die nicht signifikante Differenzierungsleistung in der Bedingung spontanen Entscheidens von Experiment 2 und die nicht-Überprüfbarkeit der Hypothese anhand der Ergebnisse von Experiment 1. Für die Ergebnisse von Experiment 2 konnte eine Erklärung gefunden werden, die auf dem potenziellen Einfluss unterschiedlicher Aufgabenstellungen zwischen den Experimenten 1 und 2 auf der einen und Experiment 3 auf der anderen Seite basiert. Diese Erklärung weist jedoch momentan den Status einer Spekulation auf und müsste daher zunächst empirisch überprüft werden. Dies wäre z.B. durch ein Experiment möglich, bei dem die unterschiedlichen Aufgabenstellungen gegeneinander getestet werden. Eine Gruppe von Versuchspersonen hätte dann Testfälle hinsichtlich der Wahrscheinlichkeit ihrer Zugehörigkeit zur instruierten Kategorie zu beurteilen, während eine andere Gruppe beurteilen müsste, ob die präsentierten Testfälle zur instruierten Kategorie oder zu einer Gegenkategorie gehören. Auf Grundlage der hier vorgebrachten Spekulation wäre zu erwarten, dass die Versuchspersonen in der ersten Gruppe auch in der Bedingung spontanen Entscheidens Kategorisierungsurteile entsprechend dem instruierten Kausalsystem treffen kann, während dies in der anderen Gruppe nicht der Fall ist. Eine empirische Bestätigung der vorgebrachten Erklärung könnte dann den Ausgangspunkt für eine neuerliche Überprüfung von Hypothese 1 bilden, um sie endgültig bestätigen zu können.

\subsection{Bewertung von Hypothese 2 anhand der Ergebnisse der Interventionsexperimente}

Im nun folgenden Abschnitt werden die Resultate der Experimente mit Interventionsaufgaben gemeinsam diskutiert. Die Ergebnismuster sind wie schon diejenigen der Kategorisierungsaufgaben nicht eindeutig und bedürfen daher einer Erläuterung. Die für 
Interventionsaufgaben aufgestellte Hypothese 2 besagt, dass eine Beteiligung von Typ 2Verarbeitungsprozessen notwendig ist, um zu korrekten Urteilen zu gelangen. Ein korrektes Urteil liegt immer dann vor, wenn von den beiden möglichen Interventionen diejenige gewählt wird, die die größere Anzahl von Variablen beeinflussen kann. Die Hypothese resultiert in der Vorhersage, dass die Verarbeitungsbedingungen, in denen die Versuchspersonen bewusst über die zu wählende Intervention nachdenken konnten zu besseren Leistungen führen sollten als die übrigen untersuchten Verarbeitungsbedingungen. Die Ergebnisse der durchgeführten Experimente mit Interventionsaufgaben (Experiment 1, 2 und 4) ergeben folgendes Bild: In Experiment 1 konnten die Vorhersagen von Hypothese 2 bestätigt werden, wenn man davon ausgeht, dass die Versuchspersonen keine Unterschiede zwischen Fällen mit gleicher zu bevorzugender Intervention machen. Die Versuchspersonen in der Bedingung bewussten Nachdenkens differenzierten im Vergleich zu den Versuchspersonen in der Bedingung spontanen Entscheidens signifikant stärker zwischen denjenigen Fällen, für die auf Grundlage des instruierten Kausalmodells unterschiedliche Vorhersagen hinsichtlich der $\mathrm{zu}$ wählenden Intervention gemacht wurden. Die fehlende Differenzierung zwischen Fällen mit gleicher zu präferierender Intervention kann wie folgt erklärt werden. Der Unterschied zwischen den beiden Fällen bestand in der Beeinflussbarkeit einer einzelnen zusätzlichen Variablen durch die zu präferierende Intervention. Diese Variable hatte zudem den niedrigsten kausalen Status im gesamten Kausalsystem und sollte daher auch einen geringen Einfluss auf die Interventionsentscheidung ausüben. Insgesamt kann somit festgehalten werden, dass diejenigen Versuchspersonen, die ihre Entscheidungen auf Basis von Typ 2-Verarbeitungsprozessen treffen konnten in der Lage waren, das zuvor erlernte Kausalwissen für Interventionsurteile zu nutzten. Zudem waren sie dabei besser als die Versuchspersonen in den anderen Verarbeitungsbedingungen.

Die Versuchspersonen in den Interventionsbedingungen von Experiment 2 waren hingegen in keiner der Verarbeitungsbedingungen in der Lage, die auf Grundlage des instruierten Kausalsystems korrekten Interventionsurteile zu fällen. Es konnten zudem keinerlei Hinweise auf Unterschiede zwischen den Verarbeitungsbedingungen gefunden werden. Hypothese 2 konnte somit durch die Ergebnisse von Experiment 2 nicht bestätigt werden.

Die Analyse der Interventionsbedingungen von Experiment 4 ergab hingegen gute Differenzierungsleistungen in den Bedingungen spontanen Entscheidens und bewussten Nachdenkens, wenn wie schon bei der Interpretation der Ergebnisse von Experiment 1 davon 
ausgegangen wird, dass die Versuchspersonen nicht zwischen Fällen mit gleicher zu präferierender Intervention unterschieden. Die guten Differenzierungsleistungen in der Bedingung spontanen Entscheidens sprechen gegen die in Hypothese 2 behauptete Notwendigkeit von Typ 2-Verarbeitungsprozessen bei Interventionsaufgaben, wenn man davon ausgeht, dass Urteile in dieser Bedingung auf Typ 1-Prozessen basieren. Entgegen der aus Hypothese 2 abgeleiteten Vorhersage zeigte sich zudem keine Überlegenheit der Differenzierungsleistung in der Bedingung bewussten Nachdenkens gegenüber der Bedingung spontanen Entscheidens.

Die vorgestellten Ergebnisse werfen zwei wesentliche Fragen auf, für die im Folgenden Antworten auf Grundlage der Unterschiede zwischen den Experimenten vorgeschlagen werden sollen. Erstens soll untersucht werden, warum es den Versuchspersonen in Experiment 2 in keiner der Verarbeitungsbedingungen gelang, zwischen den Fällen mit unterschiedlichen zu präferierenden Interventionen zu differenzieren. Zweitens soll diskutiert werden, warum die Versuchspersonen in der Bedingung spontanen Entscheidens von Experiment 4 ebenso erfolgreich zwischen den Fällen mit unterschiedlicher $\mathrm{zu}$ präferierender Intervention differenzierten wie die Versuchspersonen in der Bedingung bewussten Nachdenkens.

Die verwendeten Kausalsysteme in den drei Experimenten mit Interventionsaufgaben hatten zwar eine unterschiedliche Struktur, wiesen aber eine ähnliche Komplexität auf. In jedem der drei verwendeten Kausalsysteme gab es sowohl gemeinsame Ursache-, als auch gemeinsame Effekt-Strukturen, sowie kausale Ketten. Das Kausalsystem in Experiment 1 bestand aus 8 Variablen, die durch 8 Kausalrelationen miteinander verbunden waren. In Experiment 2 waren 10 Variablen über 10 Kausalrelationen miteinander verbunden. Das Kausalsystem in Experiment 4 wies 9 Variablen auf, die über 9 Kausalrelationen miteinander in Beziehung standen. In allen drei Experimenten wurden fiktive Coverstories genutzt, um das Kausalsystem zu vermitteln. In Experiment 1 wurden hierzu Fähigkeiten und Techniken von Schamanenschülern verwendet, in Experiment 2 repräsentierte eine Warane betreffende Krankheit das Kausalsystem. In Experiment 4 bestand die Coverstory in den Beziehungen in der Angebotspolitik für Investmentfonds bei Schweizer Kantonalbanken. Es wurde bei allen Coverstories darauf geachtet, dass sie die jeweiligen Kausalrelationen plausibel erscheinen lassen. Zudem sollten sie Phänomene beschreiben, mit denen die Versuchspersonen wenig bis keine Erfahrungen haben. Die in Experiment 2 bestehende und bereits im Abschnitt über die Kategorisierungsaufgaben beschriebene Verwechslungsgefahr bezüglich der Benennung der 
beiden Variablen mit dem höchsten kausalen Status (,Adenobakterien“ und „Coronabakterien“) sollte sich nicht auf die Interventionsurteile auswirken. Der Grund hierfür ist, dass die beiden möglichen Interventionen auf Variablen wirken, die diesen beiden Variablen in der kausalen Struktur untergeordnet sind und eine Intervention die kausale Verbindung zwischen einer Variablen und den sie normalerweise verursachenden Variablen kappt (vgl. Abschnitt 2.1.5). Insgesamt gibt es somit keine offensichtlichen Gründe dafür, dass die kausale Struktur oder die Coverstory von Experiment 2 für die mangelnde Differenzierungsleistung verantwortlich sein könnte.

Ein möglicherweise relevanter Unterschied zwischen Experiment 2 auf der einen und den Experimenten 1 und 4 auf der anderen Seite besteht in der Unterschiedlichkeit der Folgen der beiden möglichen Interventionen. Diese Unterschiedlichkeit kann z.B. als Differenz in der Anzahl der durch die beiden Interventionen beeinflussbaren Variablen gemessen werden. In Experiment 2 wurde durch die zu präferierenden Intervention in allen verwendeten Testfällen genau eine Variable mehr beeinflusst als durch die nicht zu präferierende Intervention. Diese geringstmögliche Überlegenheit der zu präferierenden Intervention kann bereits bei kleinen Fehlern in der Repräsentation des präsentierten Falles zu Fehlurteilen führen. Solche Fehler können entweder bereits während der Informationsaufnahme (z.B. wenn eine der Variablenausprägungen während der Stimuluspräsentation nicht korrekt wahrgenommen wird) oder bei der Integration der beobachteten Variablenausprägung mit dem gelernten Kausalsystem (z.B. wenn eine Ausprägung einer falschen Position im System zugeordnet wird) entstehen. Der Grund für diese geringen Unterschiede liegt in den besonderen Anforderungen an die Testfälle in Experiment 2. Zum einen sollten die Fälle geeignet sein, um mit identischen Kombinationen von Variablenausprägungen sowohl Kategorisierungs- als auch Interventionsurteile bei unterschiedlichen Versuchspersonen abfragen zu können. Daher mussten die Testfälle auch die an diese beiden Arten von Urteilen gestellten Anforderungen erfüllen. In Bezug auf die Kategorisierungsurteile bestanden diese in einer in allen Fällen gleichen Anzahl von für die instruierte Kategorie charakteristischen Variablenausprägungen. Zudem sollte die Wirkung der kausalen Faktoren (kausaler Status und kausale Kohärenz) für jeden einzelnen Fall jeweils die gleichen Vorhersagen zur Folge haben. So sollte ein Fall, in dem die Variablen mit dem höchsten kausalen Status die für die instruierte Kategorie charakteristischen Ausprägungen besitzen, zugleich eine hohe kausale Kohärenz aufweisen, so dass auf Grundlage beider Faktoren ein hohes Urteil bezüglich der Kategorienzugehörigkeit zu erwarten ist. In Bezug auf die Interventionsurteile musste zum einen sichergestellt werden, dass die beiden Variablen, auf die die beiden möglichen 
Interventionen wirken, in allen Fällen diejenige Ausprägung besaßen, die von der jeweiligen Intervention geändert werden konnte. Im Fall der Warane betreffenden Torokrankheit bedeutete dies, dass die Interventionen nur auf vorhandene Symptome wirken konnten. Aufgrund dieser Restriktion fielen die beiden Variablen mit dem höchsten kausalen Status als mögliche Interventionspunkte aus, da diese ja aufgrund der Testfälle für die Kategorisierungsurteile unterschiedliche Ausprägungen in den verschiedenen Fällen aufwiesen. Zudem musste sichergestellt werden, dass die beiden möglichen Interventionen in allen Fällen auf dieselben Variablen wirken, um keinen zusätzlichen und im Vergleich zu den Kategorisierungsaufgaben wesentlich höheren Lernaufwand bei den Versuchspersonen zu erzeugen. Zuletzt mussten die Testfälle so konstruiert werden, dass sich auf Grundlage des Kausalsystems stets eine eindeutige, wenn auch knappe, Präferenz für eine der beiden Interventionsoptionen ergab.

Die Unterschiedlichkeit der Interventionsfolgen war in den beiden anderen Experimenten deutlich stärker ausgeprägt. Der über alle Fälle gemittelte Unterschied in der Anzahl der durch die beiden Interventionen beeinflussbaren Variablen lag bei 2,25 in Experiment 1 und bei 1,71 in Experiment 4. In diesen Experimenten hatten die Versuchspersonen somit im Vergleich zu Experiment 2 mehr Anhaltspunkte, um sich für eine der beiden möglichen Interventionen zu entscheiden. Dieser Faktor könnte den Ausschlag dafür gegeben haben, dass die Versuchspersonen in Experiment 2 nicht zwischen den Fällen mit unterschiedlichen auf Grundlage des instruierten Kausalmodells vorhergesagten Interventionen differenzieren konnten.

Wie bereits weiter oben erläutert, waren die Ähnlichkeit zwischen den drei Experimenten, mit denen Interventionsurteile untersucht wurden, hinsichtlich kausaler Struktur und verwendeter Coverstory zu groß, um hieraus Unterschiede in den Ergebnissen erklären zu können. Bei der Betrachtung der Frage, warum die Versuchspersonen in der Bedingung spontanen Entscheidens in Experiment 4 ebenso gut wie diejenigen in der Bedingung bewussten Denkens zwischen den Fällen mit unterschiedlicher auf Grundlage des Kausalsystems zu präferierender Intervention differenzieren konnten, fallen jedoch zwei weitere Unterschiede zwischen den Experimenten auf. Der erste Unterschied bezieht sich auf die Vermittlung des verwendeten Kausalsystems. Den Versuchspersonen in Experiment 4 wurde das Kausalsystems ausschließlich mittels Text, also ohne graphische Darstellungen wie in den Experimenten 1 und 2, vermittelt. Zudem wurden alle Parameter des Kausalsystems (Basisraten der Variablen und Kausalstärken zwischen den Variablen) in Experiment 4 als 
Prozentangaben präsentiert, während sie in den beiden anderen Experimenten zumindest teilweise verbal umschrieben wurden (In Experiment 1 wurden die Basisraten ebenfalls mit Prozentangaben vermittelt). Die Form der Wissensvermittlung in Experiment 4 macht es plausibel anzunehmen, dass das Kausalsystem hier relativ schwer zu erlernen war. Hinweise hierauf sind zum einen der mit $28 \%$ relativ hohe Anteil an Versuchspersonen, die am Multiple-Choice-Wissenstest von Experiment 4 scheiterten. Zum anderen war die Anzahl der Lerndurchgänge, die die Versuchspersonen im Durchschnitt benötigten, die innerhalb der maximal möglichen 6 Durchgänge alle Fragen des Multiple-Choice-Wissenstests korrekt beantworten konnten in Experiment 4 höher als in den beiden anderen Experimenten mit Interventionsaufgaben. Während die Versuchspersonen in den Interventionsbedingungen der Experimente 1 und 2 hierfür 1,71 bzw. 2,44 Lerndurchgänge benötigten, ergab sich ein Durchschnitt von 3,28 Durchgängen in Experiment 4. Diese Zahlen können zwar aufgrund der inhaltlichen und strukturellen Unterschiede zwischen den Experimenten nicht statistisch miteinander verglichen werden, sie geben aber dennoch einen Anhaltspunkt dafür, dass die Versuchspersonen in Experiment 4 etwas länger zum Erlernen des Kausalsystems brauchten. Es ist jedoch fraglich, ob und wenn ja, wie sich ein schwerer zu erlernendes Kausalsystem in positiver Weise auf Urteile niederschlagen soll, die mittels Typ 1-Verarbeitungsprozessen gebildet werden. Daher kann nicht davon ausgegangen werden, dass die geschilderten Besonderheiten bei der Vermittlung des verwendeten Kausalsystems in Experiment 4 zur besseren Differenzierungsleistung in der Bedingung der spontanen Verarbeitung beigetragen haben.

Der zweite wesentliche Unterschied zu den beiden anderen Experimenten mit Interventionsaufgaben bestand in einer Verlängerung der Dauer der Präsentation der einzelnen Variablenausprägungen. Während diese in den Experimenten 1 und 2 jeweils 1,5 Sekunden betrug wurde sie in Experiment 4 auf 2,5 Sekunden erhöht. Die längere Stimuluspräsentation war die Konsequenz aus im Vorfeld von Experiment 4 durchgeführten Vortests. Da die Präsentation der Variablenausprägungen in Form von Sätzen erfolgte, benötigten die Versuchspersonen im Vortest länger, um die Informationen aufzunehmen. Es besteht jedoch die Möglichkeit, dass sich die Versuchspersonen im eigentlichen Experiment auf die wesentlichen Bestandteile der jeweiligen Stimuluspräsentation fokussiert haben und dadurch Zeit für Typ 2-Verarbeitungsprozesse zur Verfügung stand. Ein Beispiel für die Präsentation einer Variablenausprägung ist „Die Kantonalbank Chur hat den Fonds EuroInvest im Angebot“. Da die Informationen für einen einzelnen Fonds in einem Block präsentiert wurden, woraufhin die Manipulation der Verarbeitungsbedingungen, sowie die 
Abfrage des Urteils für diesen Fonds erfolgte, war es nicht notwendig, den Namen des Fonds zu beachten. Die relevanten Informationen bestanden im Namen der Stadt (hier: Chur), sowie der An- bzw. Abwesenheit des Wortes „NICHT“ im Anschluss an den Fondsnamen (hier: abwesend), das zudem in Großbuchstaben präsentiert wurde. Wenn man davon ausgeht, dass sich die Versuchspersonen während der Stimuluspräsentation auf die Aufnahme dieser beiden Informationseinheiten beschränkten, so kann davon ausgegangen werden, dass sie weniger als die vorhandenen 2,5 Sekunden hierfür benötigten. Die verbleibende Zeit kann dann von Typ 2-Prozessen dazu genutzt worden sein, bereits während der Stimuluspräsentation an der Lösung der Aufgabe zu arbeiten. Dies gilt insbesondere für die Bedingung des spontanen Entscheidens, bei der die Versuchspersonen wussten, dass sie unmittelbar im Anschluss ihr Urteil bezüglich der beiden möglichen Interventionen abzugeben hatten. Es besteht somit die Möglichkeit, dass bereits während der Stimuluspräsentation eine bewusste OnlineVerarbeitung stattfand, die Ähnlichkeiten zur Bedingung der Online-Verarbeitung bei den Kategorisierungsaufgaben von Experiment 3 aufweist. Es gab beim hier betrachteten Experiment zwar immer noch Restriktionen bezüglich der Dauer der Betrachtung der einzelnen Variablenausprägung, aber das festgelegte 2,5-Sekunden-Intervall ist nur unwesentlich kürzer als die durchschnittliche selbstgewählte Betrachtungszeit in Experiment 3, die 2,86 Sekunden betrug. Ein direkter Vergleich zwischen den Experimenten 3 und 4 ist aufgrund der Unterschiede in der Art der zu bearbeitenden Aufgabe natürlich nicht möglich. Während in Experiment 3 Kategorisierungsaufgaben zu lösen waren, sollten in Experiment 4 Interventionen beurteilt werden. In der vorliegenden Arbeit wird angenommen, dass Kategorisierungsaufgaben auch ohne den Einfluss von Typ 2-Verarbeitungsprozessen vorgenommen werden können, diese aber für erfolgreiche Interventionsurteile notwendig sind. Die Ergebnisse von Experiment 3 können als Belege für die Annahme bezüglich der Kategorisierungsaufgaben angesehen werden. Die Interpretation der Ergebnisse in Experiment 4 ist insofern schwieriger, als dass aufgrund der oben vorgebrachten Argumente Zweifel daran bestehen, dass das Urteil in der Bedingung spontanen Entscheidens ausschließlich auf Typ 1-Verarbeitungsprozessen basiert.

Es wäre daher für eine abschließende Beurteilung der Hypothese 2 erforderlich, empirisch zu überprüfen, ob die vorgebrachte Erklärung einer Typ 2-Online-Verarbeitung belegt werden kann, oder ob die Versuchspersonen tatsächlich in der Lage sind, auch Interventionsurteile ohne die Anwesenheit von Typ 2-Verarbeitungsprozessen korrekt zu fällen. Eine Möglichkeit für eine solche Überprüfung bestünde beispielsweise in einem Experiment mit Interventionsaufgaben, bei dem die Vermittlung des Kausalsystems 
derjenigen in Experiment 1 entspricht. Die Dauer der Darbietung der einzelnen Variablenausprägungen könnte dann wieder den in den anderen Experimenten genutzten Wert von 1,5 Sekunden annehmen. Neben einem Test in den Bedingungen des spontanen Entscheidens und des Entscheidens mit bewusstem Nachdenken könnte in einer zusätzlichen Bedingung der Online-Verarbeitung geprüft werden, ob längere und selbstgewählte Präsentationszeiten der Variablenausprägungen zum einen zu besseren Ergebnissen als in der Bedingung spontanen Entscheidens und zudem zu vergleichbaren Ergebnissen wie in der Bedingung bewussten Denkens führen. Darüber hinaus könnte eine weitere Bedingung des spontanen Entscheidens durchgeführt werden, in der sich die festgelegte Präsentationsdauer an der durchschnittlichen selbstgewählten Dauer in der Bedingung der Online-Verarbeitung orientiert. Der Vergleich der Ergebnisse dieser Bedingung mit denjenigen der Bedingung der Online-Verarbeitung würde die Exploration des möglichen Einflusses eigener Kontrolle während der Stimuluspräsentation auf die ablaufenden Verarbeitungsprozesse ermöglichen.

Auf Grundlage der in den Experimenten 1, 2 und 4 erhaltenen Ergebnisse muss die aufgestellte Hypothese 2 jedoch als nicht bestätigt bewertet werden, da die Befundlage zu uneinheitlich ist. Während die Hypothese in Experiment 1 in vollem Umfang bestätigt werden konnte, war dies in den Experimenten 2 und 4 nicht der Fall. In Experiment 2 lag dies an der nicht vorhandenen Differenzierung zwischen Fällen mit unterschiedlicher auf Grundlage des instruierten Kausalsystems $\mathrm{zu}$ präferierender Intervention in sämtlichen Verarbeitungsbedingungen. Hierfür konnte eine Erklärung angeboten werden, die auf den besonderen Anforderungen bei der Konstruktion der Testfälle beruht. Experiment 4 erbrachte hingegen ein Ergebnis, das der Annahme einer Notwendigkeit von Typ 2Verarbeitungsprozessen in Hypothese 2 direkt $\mathrm{zu}$ widersprechen scheint. Die Versuchspersonen waren sowohl in der Bedingung spontanen Entscheidens als auch in der Bedingung bewussten Denkens in der Lage, zwischen den Fällen mit unterschiedlicher zu präferierender Intervention zu differenzieren. Zudem wurden keine Unterschiede zwischen diesen Bedingungen festgestellt. Die für das Zustandekommen der Ergebnisse vorgebrachte Erklärung des Wirkens von Typ 2-Verarbeitungsprozessen bereits während der Stimuluspräsentation bedarf jedoch erst einer empirischen Überprüfung um die Unvereinbarkeit der Ergebnisse mit Hypothese 2 aufzulösen.

Die Ergebnisse von Experiment 5 mit dem mentale Simulationsprozesse ohne den externen Einfluss durch Interventionen untersucht wurden, sollen ebenfalls an dieser Stelle diskutiert werden. Der Grund hierfür liegt in der Annahme, dass für mentale Simulationen, 
die auch einen wichtigen Bestandteil von Interventionsentscheidungen bilden, ebenfalls Typ 2-Verarbeitungsprozesse erforderlich sind (vgl. Abschnitt 2.4.3.2). Die Ergebnisse von Experiment 5 zeigten jedoch keine Überlegenheit in den Differenzierungsleistungen in den beiden Bedingungen, in denen die Versuchspersonen bewusst über die Lösung der Aufgabe nachdenken konnten. Die Vermutung einer Notwendigkeit von Typ 2-Verarbeitungsprozessen bei mentalen Simulationen in kausalen Systemen konnte somit nicht bestätigt werden. Die vorhandenen signifikanten Differenzierungen in allen Bedingungen außer der des spontanen Entscheidens deuten zwar an, dass zusätzlich zur Verfügung stehende Zeit nach der Stimuluspräsentation hilfreich für die Urteilsbildung sein kann, sie aber nicht unbedingt zum bewussten Nachdenken über die Aufgabe genutzt werden muss.

Ein Grund für die nicht nachweisbare Überlegenheit der Bedingungen, die bewusstes Nachdenken erlaubten, könnte in der großen Menge der während der Stimuluspräsentation dargebotenen Informationen gelegen haben. Die Versuchspersonen wurden während der Stimuluspräsentation mit 4 Fällen à 8 Variablenausprägungen konfrontiert und hatten sich Urteile für jeden der Fälle zu bilden. Die begrenzte Kapazität des Arbeitsgedächtnisses (vgl. Dijksterhuis \& Nordgren, 2006), auf dessen Grundlage die Verarbeitung mittels Typ 2Prozessen erfolgt (vgl. Abschnitt 3.2), könnte dazu geführt haben, dass die Differenzierungsleistung der Versuchspersonen in den Bedingungen bewussten Nachdenkens nicht besser war als in den übrigen Bedingungen.

\subsection{Unbewusstes Denken bei Kausalurteilen?}

In Abschnitt 3.2 wurde diskutiert, ob sich die von Dijksterhuis und Nordgren (2006) postulierten Vorteile unbewussten Denkens auch auf den Bereich des kausalen Urteilens- und Entscheidens übertragen lassen. In den im Rahmen dieser Arbeit durchgeführten Experimenten wurde daher auch geprüft, ob eine Phase unbewussten Denkens einen Einfluss auf im Anschluss zu treffende Urteile ausüben kann. Für diejenigen Experimente, in denen Kategorisierungsaufgaben untersucht wurden gestaltet sich die Interpretation der Ergebnisse im Hinblick auf die Differenzierungsleistungen in den Bedingungen unbewussten Denkens problematisch. Die Ergebnisse von Experiment 1 können aufgrund der plausiblen Alternativerklärung nicht für die Untersuchung einer Anwendung kausalen Wissens beim unbewussten Denken herangezogen werden (vgl. Abschnitt 4.2.4.2). Darüber hinaus ergaben sich widersprüchliche Befunde in den Bedingungen unbewussten Denkens von Experiment 2 und 3. Während die Versuchspersonen in dieser Bedingung in Experiment 2 marginal signifikante Differenzierungsleistungen erbrachten, konnten sie in Experiment 3 nicht 
zwischen den Fällen mit unterschiedlichem erwarteten Kategorisierungsurteil unterscheiden. Im Sinne zeitintensiver unbewusst ablaufender Denkprozesse (vgl. Dijksterhuis \& Nordgren, 2006) könnten zwar die Ergebnisse von Experiment 2, nicht aber diejenigen von Experiment 3 erklärt werden. Darüber hinaus waren die Differenzierungsleistungen in der spontanen Bedingung von Experiment 3 signifikant besser (und in den beiden Bedingungen, die Typ 2Prozesse ermöglichten marginal signifikant besser) als diejenigen in der Bedingung unbewussten Denkens. Dieses Ergebnis deutet auf eine negative Beeinflussung eines während der Stimuluspräsentation gebildeten Urteils durch die Distraktoraufgabe in der Bedingung unbewussten Denkens hin und steht damit dem Top-Down versus Bottom-Up-Prinzip der UTT (vgl. Abschnitt 3.2) entgegen. Insgesamt kann der Widerspruch zwischen den Ergebnissen in den Bedingungen unbewussten Denkens zwar nicht aufgelöst werden, die eindeutigeren Ergebnisse in Experiment 3 deuten jedoch eine zufällig zustande gekommene Differenzierungsleistung in dieser Bedingung von Experiment 2 an.

Zusammenfassend kann daher aufgrund der Befundlage in den Experimenten mit Kategorisierungsaufgaben nicht davon ausgegangen werden, dass sich die Vorteile des unbewussten Denkens auf den Bereich der kausalen Kategorisierungen übertragen lassen. Diese Annahme wird noch durch folgenden Umstand bestärkt: Obwohl die zu bewältigenden Aufgaben aufgrund der Komplexität der verwendeten Kausalsysteme geeignet erschienen, um mögliche Vorteile des unbewussten Denkens festzustellen, konnte in keinem der Experimente eine Überlegenheit gegenüber den anderen getesteten Verarbeitungsprozessen nachgewiesen werden.

Die Ergebnisse in den Bedingungen unbewussten Denkens für die Experimente mit Interventionsaufgaben sind eindeutig. In keinem Experiment waren die Versuchspersonen in dieser Bedingung in der Lage, zwischen den Fällen mit unterschiedlicher zu präferierender Intervention zu differenzieren. In Experiment 1 waren die Differenzierungsleistungen in dieser Bedingung marginal signifikant schlechter als in der Bedingung des bewussten Denkens, in Experiment 4 differenzierten die Versuchspersonen in der Bedingung bewussten Denkens signifikant und diejenigen in der Bedingung spontanen Entscheidens marginal signifikant besser als die Versuchspersonen in der Bedingung unbewussten Denkens. In Experiment 2 konnten die Versuchspersonen in der Bedingung unbewussten Denkens ebenso wie diejenigen in den anderen experimentellen Bedingungen nicht zwischen den Fall-Clustern mit unterschiedlichem vorhergesagten Interventionsurteil differenzieren. Insgesamt kann also 
auf Grundlage der Ergebnisse für Interventionsaufgaben davon ausgegangen werden, dass unbewusstes Denken hier zu keinerlei Verbesserungen der Leistungen führt.

Die Durchführung von Experiment 5 hatte neben der im vorangegangenen Abschnitt geschilderten Untersuchung mentaler Simulationsprozesse das Ziel, Bedingungen zu schaffen, die denen in klassischen Experimenten zur Unconscious Thought Theory (Dijksterhuis \& Nordgren, 2006) möglichst ähnlich sind. Dadurch sollten möglichst günstige Voraussetzungen für das Wirken unbewusster Denkprozesse auf dem Gebiet des Urteilens auf Grundlage kausalen Wissens geschaffen werden. Wie in den vorangegangen Abschnitten bereits diskutiert, führte unbewusstes Denken in keinem zur Testung der aufgestellten Hypothesen 1 und 2 durchgeführten Experimente 1 bis 4 zu überlegenen Leistungen der Versuchspersonen, meistens erbrachte es sogar gar keine Effekte.

Die Versuchspersonen in der Bedingung des unbewussten Denkens von Experiment 5 waren zwar in der Lage, erfolgreich zwischen den Fall-Clustern mit unterschiedlichen vorhergesagten Ratings zu differenzieren. Allerdings waren sie dabei nicht besser als in den anderen Verarbeitungsbedinungen. Daher kann die durch die Unconscious Thought Theory (Dijksterhuis \& Nordgren, 2006) vorhergesagte und empirisch auch nachgewiesene Überlegenheit des unbewussten Denkens (z.B. Dijksterhuis et al., 2006) gegenüber dem bewussten Denkens auch in Experiment 5 nicht auf dem Gebiet des kausalen Urteilens und Entscheidens nachgewiesen werden. Insgesamt sprechen die Befunde der im Rahmen dieser Arbeit vorgestellten Experimente somit eindeutig gegen die Übertragbarkeit der UTT auf das Gebiet des kausalen Urteilens und Entscheidens. Es ist jedoch anzumerken, dass mit dieser Aussage keine Beurteilung der Gültigkeit der UTT im Allgemeinen abgegeben werden soll. Der Fokus dieser Arbeit richtet sich auf das kausale Urteilen und Entscheiden und in diesem Bereich scheint die Theorie nicht anwendbar zu sein.

\subsection{Konklusion}

Die vorliegende Arbeit hatte zum Ziel, Urteile auf Basis kausalen Vorwissens hinsichtlich der ihnen zu Grunde liegenden kognitiven Verarbeitungsprozesse zu untersuchen. Es wurden zwei Hypothesen aufgestellt und empirisch geprüft, die auf der Annahme basieren, dass unterschiedliche Arten von Aufgaben im Bereich des kausalen Urteilens und Entscheidens unterschiedliche Arten von Verarbeitungsprozessen erfordern. Die Verarbeitungsprozesse wurden auf Grundlage der in anderen Bereichen der Kognitionswissenschaft vielfach beforschten Zwei-Prozess-Ansätze in zwei Kategorien 
eingeteilt (vgl. Evans, 2008). Verarbeitungsprozesse vom Typ 1 wurden z.B. als schnell, unbewusst, automatisch und intuitiv charakterisiert, während Typ 2-Verarbeitungsprozesse z.B. als langsam, bewusst und willentlich ausführbar verstanden wurden. Ausgehend von einer Analyse desjenigen Ausschnitts der Literatur zu kausalen Phänomenen, die Rückschlüsse auf kognitive Verarbeitungsprozesse erlaubt, wurde vorhergesagt, dass kausale Kategorisierungsaufgaben auch mittels Typ 1-Verarbeitungsprozessen erfolgreich bearbeitet werden können (Hypothese 1), während die Beurteilung möglicher Interventionen in ein kausales System die Beteiligung von Typ 2-Verarbeitungsprozessen erfordert (Hypothese 2). Die Ergebnisse der durchgeführten Experimente ergaben ein uneinheitliches Bild.

Hypothese 1 konnte mit Einschränkung bestätigt werden. Experiment 3 zeigte, dass es möglich sein kann, korrekte kausale Kategorisierungsurteile bereits unmittelbar nach der Stimuluspräsentation abzugeben, die auch durch die Ermöglichung von Typ 2Verarbeitungsprozessen nicht verbessert werden konnten. Die Ergebnisse der ebenfalls Kategorisierungsurteile untersuchenden Experimente 1 und 2 schränken diesen Befund zwar ein, es wurden jedoch Erklärungen vorgebracht, die die Abweichungen erklären sollen.

Hypothese 2 konnte hingegen nicht bestätigt werden. Zwar gab es auch hier mit den Ergebnissen von Experiment 1 Belege, die zeigten, dass bewusstes Nachdenken spontanem Entscheiden überlegen sein kann. Diese konnten jedoch in den Experimenten 2 und 4 nicht repliziert werden. Die Ergebnisse von Experiment 4, in dem Versuchspersonen bereits sofort nach der Stimuluspräsentation korrekte Interventionsurteile fällen konnten, die sich qualitativ nicht von denen in der Bedingung bewussten Denkens unterschieden, widersprechen der Hypothese sogar eindeutig. Es wurden jedoch auch hier Erklärungen präsentiert, die das Zustandekommen der Ergebnisse erklären sollen und sie mit der aufgestellten Hypothese vereinbar machen würden.

In Bezug auf die Positionierung kausaler Phänomene in der Zwei-Prozess-Debatte kann somit festgehalten werden, dass Kategorisierungen auf Grundlage kausalen Vorwissens offenbar auch aus Typ 1-Verarbeitungsprozessen resultieren können. Die Befunde lassen sich somit mit den Annahmen derjenigen Theorien vereinbaren, die davon ausgehen, dass kausale Phänomene auch intuitiv verarbeitet werden können (z.B. Kahneman \& Frederick, 2002; Kahnemann, 2011). Sie stellen somit eine Herausforderung für diejenigen Ansätze dar, die eine Beteiligung von Typ 2-Verarbeitungsprozessen an der Durchführung kausaler Kategorisierungen als unabdingbar erachten (z.B. Sloman, 1996). Zudem konnte eine Notwendigkeit von Typ 2-Verarbeitungsprozessen zur erfolgreichen Bearbeitung von 
Interventionsaufgaben nicht zweifelsfrei nachgewiesen werden. Dieser Befund widerspricht den Annahmen der im Rahmen dieser Arbeit analysierten Theorien (z.B. Lagnado, 2011; Sloman \& Hagmayer, 2006) und der aufgestellten Hypothese 2. Die Befundlage ist jedoch nicht eindeutig, so dass es weiterer Überprüfung bedarf, um die Rolle der beiden Arten kognitiver Verarbeitungsprozesse bei der Bearbeitung von Interventionsaufgaben beurteilen zu können.

Unabhängig von der Bewertung der Hypothesen konnten mit den hier vorgestellten Experimenten Belege dafür erbracht werden, dass unterschiedliche kognitive Verarbeitungsprozesse bei der kausalen Urteils- und Entscheidungsfindung ablaufen. Dabei stellte sich jedoch heraus, dass die spezifischen Gegebenheiten der Aufgabe eine wichtige Rolle spielten. Sie führten beispielsweise dazu, dass die Versuchspersonen teilweise nicht auf Grundlage ihres kausalen Vorwissens urteilten (vgl. Experiment 1) sondern andere, nichtkausale Entscheidungsstrategien wie Tallying heranzogen. Urteilten sie kausal, so resultierte dies teils aus intuitiven und teils aus bewussten Verarbeitungsprozessen. Die Ausgangsvermutung, dass kausales Urteilen und Entscheiden sowohl intuitiv über Typ 1 Prozesse als auch explizit über Typ 2 Prozesse erfolgen kann, wurde also bestätigt. Zudem ergaben sich Belege für die Bedeutung der zu bearbeitenden Aufgaben, auch wenn es scheint, dass die Art der Aufgabe nicht den einzigen Einflussfaktor darstellt. In den ersten beiden Experimenten ergaben sich deutliche Unterschiede zwischen einer Kategorisierungs- und einer Interventionswahlaufgabe, obwohl die präsentierten Informationen identisch waren. In Experiment 4 zeigte sich aber, dass auch vermeintlich Typ 2-Prozesse erfordernde Aufgaben wie Interventionsentscheidungen mit Prozessen bearbeitet werden können, die ohne eine auf die Präsentation der relevanten Informationen folgende Phase des bewussten Nachdenkens auskommen.

Insgesamt kann somit festgestellt werden, dass die eingesetzte Methodik, die aus einer Manipulation der möglichen Denkprozesse nach der Informationsvermittlung bestand, nicht genügend Kontrolle liefert, um beim kausalen Urteilen und Entscheiden die ablaufenden kognitiven Prozesse hinreichend genau zu untersuchen. Die Befunde weisen deutlich darauf hin, dass gerade in der Phase der Informationsaufnahme bereits wichtige und vermutlich je nach Aufgabe unterschiedliche Verarbeitungsprozesse auftreten können, die über die reine Encodierung der Information hinausgehen. Diese Erkenntnis wirft ein kritisches Licht auf viele Studien (z.B. Dijksterhuis, 2004; Mamede et al., 2010; Thompson et al., 2011), die diese Möglichkeit nicht in Betracht ziehen. Daher wäre es in zukünftigen Studien im Sinne einer 
weiteren Differenzierung von intuitiven und expliziten kausalen Entscheidungsprozessen sinnvoll, Typ 2-Verarbeitungsprozesse bereits während der Stimuluspräsentation zu untersuchen. Dies könnte zum Beispiel auf Basis der Methode des protokollierten lauten Denkens (think aloud) geschehen (vgl. Ericsson \& Simon, 1980). Darüber hinaus könnte das Vorgehen von Versuchspersonen anhand von kritischen Testfällen wie in den Experimenten 2-5 nach kausalen und nicht kausalen Strategien differenziert werden. Im Anschluss könnten die Ergebnisse derjenigen Versuchspersonen, die sich auf Grundlage des instruierten Kausalsystems entscheiden, im Hinblick auf ihre zeitliche Dynamik in einer Bedingung ohne zeitliche Einschränkungen in der Informationsaufnahme und -verarbeitung separat analysiert werden, um einen klareren Blick auf die kognitiven Prozesse beim kausalen Urteilen und Entscheiden zu erlangen als es in den hier vorgestellten Experimenten der Fall war. Da verschiedene kognitive Prozesse unterschiedliche Zeiträume erfordern, sind so Rückschlüsse möglich, insbesondere wenn diese mit den Ergebnissen von kognitiven Modellierungen abgeglichen werden.

Die vorliegende Arbeit konnte nur einen ersten Einblick auf die kognitiven Prozesse liefern, die beim kausalen Urteilen und Entscheiden ablaufen. Es konnten empirische Belege für den Ablauf unterschiedlicher Verarbeitungsprozesse gefunden werden, die kausale Informationen verarbeiten und zu kausalen Urteilen führen. Die Frage nach den Bedingungen, die bestimmen, welche Arten von kognitiven Prozessen ausreichend bzw. notwendig sind, konnte zwar nicht abschließend geklärt werden, die Art der zu bearbeitenden Aufgabe konnte jedoch als ein Einflussfaktor identifiziert werden. Somit stellt diese Arbeit einen ersten Schritt in Richtung des Verständnisses derjenigen Bedingungen und kognitiven Prozesse dar, die eine kausale Urteils- und Entscheidungsfindung ermöglichen. 


\section{Zusammenfassung}

Die vorliegende Arbeit beschäftigt sich mit der Frage, welche kognitiven Verarbeitungsprozesse ablaufen, wenn Menschen kausales Wissen nutzen, um zu Urteilen und Entscheidungen zu gelangen. Zu diesem Zweck wird zunächst auf Grundlage besonderer Charakteristika kausaler Beziehungen gezeigt, dass es gerechtfertigt ist, kausales Denken als eigenständige Denkform zu verstehen. Im Anschluss werden einige Zwei-Prozess-Theorien im Hinblick auf die Berücksichtigung von Aspekten kausalen Denkens untersucht. Darunter befinden sich Theorien, die davon ausgehen, dass langsame, bewusste und kontrollierte Typ 2-Prozesse beim kausalen Urteilen und Entscheiden nötig sind (z.B. Sloman, 1996). Auf der anderen Seite stehen Ansätze (z.B. Kahneman, 2011), die davon ausgehen, dass kausale Informationen auch mittels schneller, unbewusster und automatisch ablaufender Typ 1Prozesse verarbeitet werden können. Daneben werden Ansätze aus dem Bereich des kausalen Denkens vorgestellt und in die Zwei-Prozess-Debatte eingeordnet. Hierbei ergeben sich vier Gruppen von Theorien: Einige Ansätze sind mit Urteilen auf Basis von Typ 1-Prozessen vereinbar (z.B. White, 2006a), andere erachten Typ 2-Prozesse als notwendig (z.B. Sloman \& Hagmayer, 2006). Darüber hinaus gibt es Theorien, die beide Arten von Prozessen berücksichtigen (z.B. Fugelsang \& Thompson, 2003), sowie agnostische Ansätze, die keine Aussage zu Verarbeitungsprozessen machen (z.B. Waldmann, 1996). Bei der Betrachtung ergeben sich Hinweise auf einen Zusammenhang zwischen den von den Theorien untersuchten kausalen Aufgaben und der Einordnung der angenommenen Prozesse.

Auf Grundlage der theoretischen Analyse werden zwei Hypothesen abgeleitet und ein experimentelles Paradigma entwickelt, um diese zu untersuchen. Hypothese 1 besagt, dass kausale Kategorisierungsurteile auf Grundlage von Typ 1-Prozessen getroffen werden. Nach Hypothese 2 sind für kausale Interventionsurteile hingegen Typ 2-Prozesse nötig. Diese Hypothesen werden in 5 Experimenten empirisch überprüft. Die Resultate zeigen, dass Hypothese 1 als mit Einschränkungen bestätigt angesehen werden kann, während Hypothese 2 als nicht bestätigt betrachtet werden muss. Es konnte gezeigt werden, dass die Art der zu bearbeitenden Aufgabe einen Einfluss auf die Verarbeitung ausübt. Daneben scheinen aber noch andere Bedingungen zu existieren, die zwar ebenfalls einen Einfluss auf die Verarbeitung ausüben, mit der gewählten Methodik jedoch nicht hinreichend kontrolliert werden konnten. Insgesamt konnte mit den Experimenten jedoch gezeigt werden, dass unterschiedliche kognitive Prozesse bei der Verarbeitung kausaler Informationen ablaufen und dass Urteile auf der Basis von Typ 1-Verarbeitungsprozessen möglich sind. 


\section{Literaturverzeichnis}

Acker, F. (2008). New findings on unconscious versus conscious thought in decision making: additional empirical data and meta-analysis. Judgment and Decision Making, 3, 292-303.

Ahn, W.-K., \& Kalish, C.W. (2000). The role of mechanism beliefs in causal reasoning. In R. Wilson \& F. Keil (Eds.), Cognition and Explanation. Cambridge, MA: MIT Press. Ahn, W.-K., Kalish, C.W., Medin, D.L., \& Gelman, S.A. (1995). The role of covariation versus mechanism information in causal atrribution. Cognition, 54, 299-352.

Ahn, W.-K., Kim, N.S., Lassaline, M.E., \& Dennis, M.J. (2000). Causal status as a determinant of feature centrality. Cognitive Psychology, 41, 361-416.

Barbey, A.K., \& Wolff, P. (2007). Learning causal structure from reasoning. In D. S. McNamara \& J. G. Trafton (Eds.), Proceedings of the 29th Annual Cognitive Science Society (pp. 713-718). Austin, TX: Cognitive Science Society.

Bargh, J.A. (2011). Unconscious thought theory and its discontents: A critique of the critiques. Social Cognition, 29, 629-637.

Beasley, N. (1968). The extent of individual differences in the perception of causality. Canadian Journal of Psychology, 22, 399-407.

Blakemore, S.J., Fonlupt, P., Pachot-Clouard, M., Darmon, C., Boyer, P., Meltzoff, A.N., Segebarth, C., \& Decety, C. (2001). How the brain perceives causality: an eventrelated fMRI study. Neuroreport, 12, 3741-3746.

Bos, M.W., \& Dijksterhuis, A. (2010). The benefits of "sleeping on things": Unconscious thought leads to automatic weighting. Journal of Consumer Psychology. 21, 4-8.

Buehner, M.J. (2005). Contiguity and covariation in human causal inference. Learning \& behavior, 33, 230-238.

Buehner, M.J., \& May, J. (2002). Knowledge mediates the timeframe of covariation assessment in human causal induction. Thinking \& Reasoning, 8, 269-295.

Buehner, M.J., \& May, J. (2003). Rethinking temporal contiguity and the judgement of causality: Effects of prior knowledge, experience, and reinforcement procedure. The Quarterly Journal of Experimental Psychology, 56A, 865-890.

Chaigneau, S., \& Barbey, A.K. (2008). Assessing psychological theories of causal meaning and inference. In B.C. Love, K. McRae \& V.M. Sloutsky (Eds.), Proceedings of the 30th Annual Conference of the Cognitive Science Society (S. 1111-1116). Austin, TX: Cognitive Science Society. 
Cheng, P.W. (1997). From covariation to causation: A causal power theory. Psychological Review, 104, 367-405.

Cheng, P.W., \& Holyoak, K.J. (1985). Pragmatic reasoning schemas. Cognitive Psychology, 17, 391-416.

Clark, A. (1997). Being there: Putting brain, body, and world together again. Cambridge, MA: MIT Press.

Craik, K. (1952). The Nature of Explanation. Cambridge: Cambridge Unviversity Press.

Cummins, D.D., Lubart, T., Alksnis, O., \& Rist, R. (1991). Conditional reasoning and causation. Memory \& Cognition, 19, 274-282.

Danks, D. (2009). The psychology of causal perception and reasoning. In H. Beebee, C. Hitchcock \& P. Menzies (Eds.), The Oxford handbook of causation (pp. 447-470). Oxford: Oxford University Press.

Dijksterhuis, A. (2004). Think different: The merits of unconscious thought in preference development and decision making. Journal of Personality and Social Psychology, 87, 586-598.

Dijksterhuis, A., Bos, M.W., Nordgren, L.F., van Baaren, R.B. (2006). On Making the Right Choice: The Deliberation-Without-Attention Effect. Science, 311, 1005-1007.

Dijksterhuis, A., \& Nordgren, L.F. (2006). A theory of unconscious thought. Perspectives on Psychological Science, 1, 95-109.

Dowe, P. (1992). Wesley Salmon's process theory of causality and the conserved quantity theory. Philosophy of Science, 59, 195-216.

Eells, E. (1991). Probabilistic causality. Cambridge, MA: Cambridge University Press.

Einhorn, H., \& Hogarth, R.M. (1986). Judging probable cause. Psychological Bulletin, 99, 3-19.

Ericsson, K.A., \& Simon, H.A. (1980). Verbal reports as data. Psychological Review, 87, 215-251.

Evans, J.S.B.T. (2003). In two minds: dual-process accounts of reasoning. Trends in Cognitive Sciences, 7, 454-459.

Evans, J.S.B.T. (2008). Dual-processing accounts of reasoning, judgment, and social cognition. Annual Review of Psychology, 59, 255-278.

Evans, J.S.T.B. (2009). How many dual-process theories do we need? One, two, or many?. In J.S.T.B. Evans \& K.E. Frankish (Eds.), In two minds: Dual processes and beyond (pp. 33-54). Oxford: Oxford University Press. 
Evans, J.S.B.T. (2011). Dual-process theories of reasoning: Contemporary issues and developmental applications. Developmental Review, 31, 86-102.

Evans, J.S.B.T., \& Frankish, K.E. (Eds.). (2009). In two minds: Dual processes and beyond. Oxford: Oxford University Press.

Fodor, J.A. (1983). Modularity of mind. Cambridge, MA: MIT Press.

Frankish, K.E., \& Evans, J.S.T.B. (2009). The duality of mind: An historical perspective. In J.S.T.B. Evans \& K.E. Frankish (Eds.), In two minds: Dual processes and beyond (pp. 132). Oxford: Oxford University Press.

Fugelsang, J., \& Thompson, V.A. (2003). A dual-process model of belief and evidence interactions in causal reasoning. Memory \& Cognition, 31, 800-815.

Gallistel, C.R., \& Gibbon, J. (2000). Time, rate, and conditioning. Psychological Review, $107,289-344$.

Glennan, S. (2009). Mechanisms. In H. Beebee, C. \& P. Menzies (Eds.), The Oxford Handbook of Causation (pp. 315-325). Oxford: Oxford University Press.

Glöckner, A., \& Witteman, C. (2010). Beyond dual-process models: A categorisation of processes underlying intuitive judgement and decision making. Thinking \& Reasoning, 16, 1-25.

Glymour, C. (1998). Learning causes: psychological explanations of causal explanation. Minds and Machines, 8, 39-60.

Glymour, C.N. (2001). The mind's arrows: Bayes nets and graphical causal models in psychology. Cambridge, MA: MIT Press.

Goldvarg, E., \& Johnson-Laird, P.N. (2001). Naive causality: a mental model theory of causal meaning and reasoning. Cognitive Science, 25, 565-610.

González-Vallejo, C., Lassiter, G.D., Bellezza, F.S., \& Lindberg, M.J. (2008). “Save angels perhaps": A critical examination of unconscious thought theory and the deliberationwithout-attention effect. Review of General Psychology, 12, 282-296.

Gopnik, A., Glymour, C., Sobel, D.M., Schulz, L.E., Kushnir, T., \& Danks, D. (2004). A theory of causal learning in children: Causal maps and Bayes nets. Psychological Review, $111,3-32$.

Greville, W.J., \& Buehner, M.J. (2010). Temporal predictability facilitates causal learning. Journal of Experimental Psychology: General, 139, 756-771.

Griffiths, T.L., \& Tenenbaum, J.B. (2005). Structure and strength in causal induction. Cognitive Psychology, 51, 334-384. 
Gruber, H., Fink, C., \& Damm, V. (1957). Effects of experience on perception of causality. Journal of Experimental Psychology, 53, 89-93.

Hagmayer, Y., \& Sloman, S.A. (2009). Decision makers conceive of their choices as interventions. Journal of Experimental Psychology: General, 138, 22-38.

Hagmayer, Y., \& Waldmann, M.R. (2002). How temporal assumptions influence causal judgments. Memory \& Cognition, 30, 1128-1137.

Hagmayer, Y., Meder, B., Osman, M., Mangold, S., \& Lagnado, D.A. (2010). Spontaneous causal learning while controlling a dynamic system. The Open Psychology Journal, 3 , 145-162.

Hagmayer, Y., Sloman, S.A., Lagnado, D.A., \& Waldmann, M.R. (2007). Causal reasoning through intervention. In A. Gopnik \& L. Schulz (Eds.), Causal learing: Psychology, philosophy, and computation (pp.86-100). Oxford: Oxford University Press.

Hegarty, M. (2004). Mechanical reasoning by mental simulation. Trends in Cognitive Sciences, 8, 280-285.

Hume, D. (1739/1978). A treatise of human nature. Oxford: Clarendon Press.

Johnson-Laird, P.N., Byrne, R.M., \& Schaeken, W. (1992). Propositional reasoning by model. Psychological Review, 99, 418-439.

Jonides, J., Schumacher, E.H., Smith, E.E., Lauber, E.J., Awh, E., Minoshima, S., \& Koeppe, R. A. (1997). Verbal working memory load affects regional brain activation as measured by PET. Journal of Cognitive Neuroscience, 9, 462-475.

Juslin, P., Olsson, H., \& Olsson, A.-C. (2003). Exemplar effects in categorization and multiple-cue judgment. Journal of Experimental Psychology: General, 132, 133-156.

Kahneman, D. (2011). Thinking, fast and slow. New York, NY: Farrar, Straus and Giroux.

Kahneman, D., \& Frederick, S. (2002). Representativeness revisited: Attribute substitution in intuitive judgment. In T. Gilovich, D. Griffin \& D. Kahneman (Eds.), Heuristics and biases: The psychology of intuitive judgment (pp.49-81). New York : Cambridge University Press.

Kahneman, D., \& Tversky, A. (1973). On the psychology of prediction. Psychological Review, 80, 237-251.

Kelly, H. (1973). The process of Causal Attribution. American Psychologist, 28, 107-128. Keren, G., \& Schul, Y. (2009). Two is not always better than one. Perspectives on Psychological Science, 4, 533-550.

Lagnado, D.A. (2011). Causal thinking. In P. McKay-Illari, F. Russo \& J. Williamson (Eds.), Causality in the Sciences (pp. 129-149), Oxford: Oxfor University Press. 
Lagnado, D.A., \& Sloman, S.A. (2004). The advantage of timely intervention. Journal of Experimental Psychology: Learning, Memory, and Cognition, 30, 856-876.

Lagnado, D.A., Waldmann, M.R., \& Hagmayer, Y. (2007). Beyond covariation: cues to causal structure. In A. Gopnik \& L. Schulz (Eds.), Causal learing: Psychology, philosophy, and computation (pp. 154-172), Oxford: Oxford University Press.

Luhmann, C.C., Ahn, W.-K., \& Palmeri, T.J. (2006). Theory-based categorization under speeded conditions. Memory \& Cognition, 34, 1102-1111.

Machamer, P. (2004). Activities and causation: The metaphysics and epistemology of mechanisms. International Studies in the Philosophy of Science, 18, 27-39.

Machamer, P., Darden, L., \& Craver, C.F. (2000). Thinking about Mechanisms. Philosophy of Scinece, 67, 1-25.

Mamede, S., Schmidt, H.G., \& Penaforte, J.C. (2008). Effects of reflective practice on the accuracy of medical diagnoses. Medical Education, 42, 468-475.

Mamede, S., Schmidt, H.G., Rikers, R.M.J.P., Custers, E.J.F.M., Splinter, T.A.W., \& Saase, J.L.C.M. (2010). Conscious thought beats deliberation without attention in diagnostic decision-making: at least when you are an expert. Psychological Research, 74, 586592.

Mayrhofer, R., \& Rothe, A. (in press). Causal Status meets Coherence:The Explanatory Role of Causal Models in Categorization. In N. Miyake, D. Peebles \& R.P. Cooper (Eds.), Proceedings of the 34th Annual Conference of the Cognitive Science Society. Austin, TX: Cognitive Science Society.

Meder, B., Gerstenberg, T., Hagmayer, Y., \& Waldmann, M.R. (2010). Observing and intervening: rational and heuristic models of causal decision making. The Open Psychology Journal, 3, 119-135.

Meder, B., Hagmayer, Y., \& Waldmann, M.R. (2008). Inferring interventional predictions from observational learning data. Psychonomic Bulletin \& Review, 15, 75-80.

Michotte, A.E. (1963). The perception of causality. Oxford: Basic Books.

Newell, B.R., Wong, K.Y., Cheung, J.C.H., \& Rakow, T. (2009). Think, blink or sleep on it? The impact of modes of thought on complex decision making. The Quarterly Journal of Experimental Psychology, 62, 707-732.

Newman, G.E., Choi, H., Wynn, K., \& Scholl, B.J. (2008). The origins of causal perception: Evidence from postdictive processing in infancy. Cognitive Psychology, 57, 262-291. 
Nichols, W., \& Danks, D. (2007). Decision making using learned causal structures. In D.S. McNamara \& J.G. Trafton (Eds.), Proceedings of the 29th Annual Cognitive Science Society (pp. 1343-1348). Austin, TX: Cognitive Science Society.

Nosofsky, R.M. (1986). Attention, similarity, and the identification-categorization relationship. Journal of Experimental Psychology: General, 115, 39-61.

Osman, M. (2004). An evaluation of dual-process theories of reasoning. Psychonomic Bulletin \& Review, 11, 988-1010.

Payne, J.W., Samper, A., Bettman, J.R., \& Luce, M.F. (2008). Boundary conditions on unconscious thought in complex decision making. Psychological Science, 19, 11181123.

Pearl, J. (1988). Probabilistic reasoning in intelligent systems: Networks of plausible inference. San Mateo, CA: Morgan Kaufmann Publishers.

Pearl, J. (2009). Causality: Models, reasoning, and inference. New York, NY: Camebridge University Press.

Peng, K., \& Knowles, E.D. (2003). Culture, education, and the attribution of physical causality. Personality and Social Psychology Bulletin, 29, 1272-1284.

Perales, J.C., Shanks, D.R., \& Lagnado, D. (2010). Causal representation and behavior: The integration of mechanism and covariation. The Open Psychology Journal, 3, 174-183.

Proctor, C., \& Ahn, W.-K. (2007). The effect of causal knowledge on judgments of the likelihood of unknown features. Psychonomic Bulletin \& Review, 14, 635-639.

Rehder, B. (1999). A causal model theory of categorization. In M. Hahn \& S.C. Stoness (Eds.), Proceedings of the Twenty First Annual Conference of the Cognitive Science Society (pp. 595-600). Mahwah, NJ: Lawrence Erlbaum.

Rehder, B. (2003). A causal-model theory of conceptual representation and categorization. Journal of Experimental Psychology: Learning, Memory, and Cognition, 29, 1141-1159.

Rehder, B. (2010). Causal-based categorization: A review. In B. Ross (Ed.), The Psychology of Learning and Motivation, Vol. 52 (pp. 39-116). San Diego, CA: Academic Press.

Rehder, B., \& Kim, S. (2006). How causal knowledge affects classification: A generative theory of categorization. Journal of Experimental Psychology: Learning, Memory, and Cognition, 32, 659-683. 
Rehder, B., \& Kim, S. (2010). Causal status and coherence in causal-based categorization. Journal of Experimental Psychology: Learning, Memory, and Cognition, 36, 11711206.

Rescorla, R.A., \& Wagner, A.R. (1972). A theory of Pavlovian conditioning: Variations in the effectiveness of reinforcement and nonreinforcement. In A.H. Black \& W.F. Prokasy (Eds.), Classical conditioning II: current research and theory (pp. 64-99). New York, NY: Appleton-Century-Crofts.

Rips, L.J. (1989). Similarity,typicality, and categorization. In S. Vosniadou \& A. Ortony (Eds.), Similarity and analogical reasoning (pp. 21-59). Cambridge: Cambridge University Press.

Rips, L.J. (2008). Causal thinking. In J.E. Adler \& L.J. Rips (Eds.), Reasoning: Studies of human inference and its foundation (pp. 597-631). Cambridge: Cambridge University Press.

Rips, L.J. (2011). Causation from perception. Perspectives on Psychological Science, 6, 7797.

Robinson, A.E., Sloman, S.A., Hagmayer, Y., \& Hertzog, C. K. (2010). Causality in solving economic problems. The Journal of Problem Solving, 3, 106-130.

Rosch, E., \& Mervis, C. (1975). Family resemblances: Studies in the internal structure of categories. Cognitive Psychology, 7, 573-605.

Saito, M., \& Shimazaki, T. (2011). Causal reasoning in decision making: The role of causal models and their parameters. In B Kokinov, A. Karmiloff-Smith \& N.J. Nersessian (Eds.), European Perspectives in Cognitive Science. Sofia: New Bulgarian University Press.

Salmon, W.C. (1980). Probabilistic causality. Pacific Philosophical Quarterly, 61, 50-74.

Salmon, W.C. (1984). Scientific explanation and the causal structure of the world. Princeton, NJ: Princeton University Press.

Samuels, R. (2009). The magical number two, plus or minus: Dual-process theory as a theory of cognitive kinds. In J.S.T.B. Evans \& K.E. Frankish (Eds.), In two minds: Dual processes and beyond (pp. 129-146). Oxford: Oxford University Press.

Sherman, J.W. (Ed.). (2011). Unconscious Thought. Social Cognition, 29.

Schlottmann, A. (2000). Is perception of causality modular? Trends in Cognitive Sciences, $4,441-441$.

Schlottmann, A., \& Anderson, N.H. (1993). An information integration approach to phenomenal causality. Memory \& Cognition, 21, 785-801. 
Schneider, W., \& Shiffrin, R.M. (1977). Controlled and automatic human information processing: I. Detection, search, and attention. Psychological Review, 84, 1-66.

Scholl, B.J., \& Tremoulet, P.D. (2000). Perceptual causality and animacy. Trends in Cognitive Sciences, 4, 299-309.

Sherry, D.F., \& Schacter, D.L. (1987). The evolution of multiple memory systems. Psychological Review, 94, 439-454.

Shiffrin, R.M., \& Schneider, W. (1977). Controlled and automatic human information processing: II. Perceptual learning, automatic attending and a general theory. Psychological Review, 84, 127-190.

Sloman, S.A. (1996). The empirical case for two systems of reasoning. Psychological Bulletin, 119, 3-22.

Sloman, S.A. (2005). Causal Models. New York, NY: Oxford University Press.

Sloman, S.A., \& Hagmayer, Y. (2006). The causal psycho-logic of choice. Trends in Cognitive Sciences, 10, 407-412.

Sloman, S., \& Lagnado, D.A. (2005). Do we "do"? Cognitive Science, 29, 5-39.

Smith, E.R., \& DeCoster, J. (2000). Dual-process models in social and cognitive psychology: Conceptual integration and links to underlying memory systems. Personality and Social Psychology Review, 4, 108-131.

Smith, E.E., \& Sloman, S.A. (1994). Similarity- versus rule-based categorization. Memory \& Cognition,22, 377-386.

Sober, E. (2001). Venetian sea levels, British bread prices, and the principle of the common cause. The British journal for the philosophy of science, 52, 331-346.

Spirtes, P., Glymour, C., \& Scheines, R. (2000). Causation, prediction, and search.Cambridge, MA: MIT Press.

Stanovich, K.E. (1999). Who is rational?: Studies of individual differences in reasoning. Mahwah, NJ: Lawrence Erlbaum Associates.

Stanovich, K.E. (2004). The robot's rebellion. Chicago, IL: University Of Chicago Press. Stanovich, K.E., \& Toplak, M.E. (2012). Defining features versus incidental correlates of Type 1 and Type 2 processing. Mind \& Society, 1, 3-13.

Steyvers, M., Tenenbaum, J.B., Wagenmakers, E.J. \& Blum, B. (2003). Inferring causal networks from observations and interventions. Cognitive Science, 27, 453-489.

Strack, F., \& Deutsch, R. (2004). Reflective and impulsive determinants of social behavior. Personality and Social Psychology Review, 8, 220-247. 
Strick, M., Dijksterhuis, A., \& van Baaren, R.B. (2010). Unconscious-thought effects take place off-line, not on-line. Psychological Science, 21, 484-488.

Strick, M., Dijksterhuis, A., Bos, M.W., Sjoerdsma, A., \& van Baaren, R. (2011). A metaanalysis on unconscious thought effects. Social Cognition, 29, 738-762.

Suppes, P. (1970). A probabilistic theory of causality. Amsterdam: North Holland.

Suppes, P. (1984). Probabilistic metaphysics. Oxford: Basil Blackwell.

Talmy, L. (1988). Force dynamics in language and cognition. Cognitive Science, 12, 49100.

Tenenbaum, J., \& Griffiths, T.L. (2003). Theory-based causal inference. In S. Becker, S. Thrun \& K. Obermaier (Eds.); Advances in Neural Information Processing Systems 15 (pp. 43-50). Cambridge, MA: MIT Press.

Thompson, V.A., Turner, J.A.P., \& Pennycook, G. (2011). Intuition, reason, and metacognition. Cognitive Psychology, 63, 107-140.

Tversky, A. \& Kahneman, D. (1980). Causal schemas in judgments under uncertainty. In M. Fishbein (Ed.), Progress in social psychology, Hillsdale, NJ: Lawrence Erlbaum Associates.

Verschueren, N., Schaeken, W., \& d'Ydewalle, G. (2005). A dual-process specification of causal conditional reasoning. Thinking \& Reasoning, 11, 239-278.

Waldmann, M.R. (1996). Knowledge-based causal induction. Causal learning, 34, 47-88.

Waldmann, M.R., \& Hagmayer, Y. (1995). Causal paradox: When a cause simultaneously produces and prevents an effect. In J.D. Moore \& J.F. Lehman (Eds.), Proceedings of the Seventeenth Annual Conference of the Cognitive Science Society. (pp. 425-430). Mahwah, NJ: Lawrence Erlbaum Associates.

Waldmann, M.R., \& Hagmayer, Y. (2001). Estimating causal strength: the role of structural knowledge and processing effort. Cognition, 82, 27-58.

Waldmann, M.R., \& Hagmayer, Y. (2005). Seeing versus doing: Two modes of accessing causal knowledge. Journal of Experimental Psychology: Learning, Memory, and Cognition, 31, 216-227.

Waldmann, M.R., \& Hagmayer, Y. (in press). Causal Reasoning. In D. Reisberg (Ed.), Oxford Handbook of Cognitive Psychology.

Waldmann, M.R., \& Holyoak, K.J. (1992). Predictive and diagnostic learning within causal models: asymmetries in cue competition. Journal of Experimental Psychology: General, 121, 222-236. 
Waldmann, M.R., Holyoak, K.J., \& Fratianne, A. (1995). Causal models and the acquisition of category structure. Journal of Experimental Psychology: General, 124, 181-205.

Waroquier, L., Marchiori, D., Klein, O., \& Cleeremans, A. (2010). Is it better to think unconsciously or to trust your first impression? A reassessment of unconscious thought theory. Social Psychological and Personality Science, 1, 111-118.

Wason, P., \& Evans, J. (1975). Dual processes in reasoning? Cognition, 3, 141-154.

Weir, S. (1978). The perception of motion: Michotte revisited. Perception, 7, 247-260.

White, P. (1989). A theory of causal processing. British Journal of Psychology. 80, 431454.

White, P.A. (2006a). The causal asymmetry. Psychological Review, 113, 132-147.

White, P.A. (2006b). The role of activity in visual impressions of causality. Acta Psychologica, 123, 166-185.

White, P.A. (2009). Property transmission: An explanatory account of the role of similarity information in causal inference. Psychological Bulletin, 135, 774-793.

Wolff, P. (2007). Representing causation. Journal of Experimental Psychology: General, 136, 82-111. 


\section{Anhang A - Multiple-Choice-Fragen und -Antworten in Experiment 1}

(Hinweis: Die korrekten Antworten sind fett gedruckt.)

\section{Kategorisierungsbedingungen}

Tiefgreifendes Wissen über die Regeln der Yuwipis findet man

bei genau $20 \%$ der echten Schamanen

bei genau $80 \%$ der echten Schamanen

bei genau $80 \%$ der falschen Schamanen

bei allen echten Schamanen

Die Rekapulation als Atemtechnik findet man

bei genau $20 \%$ der echten Schamanen

bei genau $20 \%$ der falschen Schamanen

bei allen falschen Schamanen

bei allen echten Schamanen

Von welchem der folgenden Faktoren wird das Beherrschen der Göttersprache beeinflusst?

Gebrauch psychedelischer Pflanzen

Wissen über die Regeln der Yuwipis

Gesang

Atemtechnik

Von welchem der folgenden Faktoren wird die Dauer der Hungermeditation beeinflusst?

Gebrauch psychedelischer Pflanzen

Wissen über die Regeln der Yuwipis

Gesang

Atemtechnik

Von welchem der folgenden Faktoren wird der Tanz beeinflusst?

Gebrauch psychedelischer Pflanzen

Wissen über die Regeln der Yuwipis

Gesang

Atemtechnik

Von welchem der folgenden Faktoren wird die Rauchtechnik beeinflusst?

Gebrauch psychedelischer Pflanzen

Wissen über die Regeln der Yuwipis

Gesang

Atemtechnik

Welche Faktoren beeinflussen den Gesang der Schamanen?

Atemtechnik und aufgeführter Tanz

Atemtechnik und Beherrschung der Göttersprache

Beherrschung der Göttersprache und aufgeführter Tanz

Atemtechnik, Beherrschung der Göttersprache und aufgeführter Tanz 
Welche Faktoren beeinflussen den Gebrauch psychedelischer Pflanzen?

Wissen über die Regeln der Yuwipis und Dauer der Hungermeditation

Dauer der Hungermediation und Rauchtechnik

Wissen über die Regeln der Yuwipis und Rauchtechnik

Wissen über die Regeln der Yuwipis, Dauer der Hungermeditation und Rauchtechnik

Welche Techniken beherrschen ECHTE Schamanen sehr viel häufiger als falsche?

Rekapulation, Trancetanz und angemessenen Gebrauch psychedelischer Pflanzen

Hyperventilation, Sonnentanz und kurze Hungermeditationen

Rekapulation, paffende Rauchtechnik und gute Beherrschung der Göttersprache

Hyperventilation, Trancetanz und rhythmischen Gesang

Welche Techniken zeichnen FALSCHE Schamanen sehr häufig aus?

Hyperventilation, unangemessener Gebrauch psychedelischer Substanzen, Trancetänze Rekapulation, unrythmischer Gesang und kurze Hungermediationen

Tiefes Wissen, inhalierende Rauchtechnik und rhythmischer Gesang

Oberflächliches Wissen der Regeln, paffende Rauchtechnik und Sonnentänze

\section{Interventionsbedingungen}

Tiefgreifendes Wissen über die Regeln der Yuwipis haben Schamanenschüler

nie

zu genau $20 \%$

zu genau $80 \%$

immer

Die Rekapulation als Atemtechnik beherrschen Schamanenschüler

nie

zu genau $20 \%$

zu genau $80 \%$

immer

Von welchem der folgenden Faktoren wird das Beherrschen der Göttersprache beeinflusst

Gebrauch psychedelischer Pflanzen

Wissen über die Regeln der Yuwipis

Gesang

Atemtechnik

Von welchem der folgenden Faktoren wird die Dauer der Hungermeditation beeinflusst?

Gebrauch psychedelischer Pflanzen

Wissen über die Regeln der Yuwipis

Gesang

Atemtechnik 
Von welchem der folgenden Faktoren wird der Tanz beeinflusst?

Gebrauch psychedelischer Pflanzen

Wissen über die Regeln der Yuwipis

Gesang

Atemtechnik

Von welchem der folgenden Faktoren wird die Rauchtechnik beeinflusst?

Gebrauch psychedelischer Pflanzen

Wissen über die Regeln der Yuwipis

Gesang

Atemtechnik

Welche Faktoren beeinflussen den Gesang der Schamanen?

Atemtechnik und aufgeführter Tanz

Atemtechnik und Beherrschung der Göttersprache

Beherrschung der Göttersprache und aufgeführter Tanz

Atemtechnik, Beherrschung der Göttersprache und aufgeführter Tanz

Welche Faktoren beeinflussen den Gebrauch psychedelischer Pflanzen?

Wissen über die Regeln der Yuwipis und Dauer der Hungermeditation

Dauer der Hungermediation und Rauchtechnik

Wissen über die Regeln der Yuwipis und Rauchtechnik

Wissen über die Regeln der Yuwipis, Dauer der Hungermeditation und Rauchtechnik

In dem Kurs Atemtechniken wird den Schamanenschülern

Rekapulation als Atemtechnik beigebracht

Hyperventilation als Atemtechnik beigebracht

Inhalation als Atemtechnik beigebracht

Hyperventilation, Trancetanz und rhythmischen Gesang

In dem Kurs Werte und Normen werden/wird den Schamanenschülern

Die Normen der Stammesältesten beigebracht

Die Regeln ihrer Urväter beigebracht

Die Göttersprache beigebracht

Die Regeln der Yuwipis näher gebracht 


\section{Anhang B - Zusätzliche Varianzanalytische Ergebnisse in Experiment 1}

Varianzanalysen auf Grundlage der aus der kausalen Kohärenz abgeleiteten Vorhersagen für die Kategorisierungsbedingungen in Experiment 1.

Tabelle B-1: Varianzanalytische Ergebnisse für das 3-Sekunden-Intervall:

\begin{tabular}{llllll} 
Effekt & SQ & FG & MSE & F & p \\
\hline Konstante & 484825,1 & 1 & 484825,1 & 749,7147 & 0,000000 \\
Verarbeitungsbedingung & 2105,6 & 2 & 1052,8 & 1,6280 & 0,207672 \\
Fehler & 29100,6 & 45 & 646,7 & & 0,000000 \\
\hline $\begin{array}{l}\text { Fall-Cluster } \\
\begin{array}{l}\text { Interaktion } \\
\text { (Verarbeitungsbedingung X }\end{array}\end{array}$ & 66095,2 & 5 & 13219,0 & 39,9098 & \\
$\begin{array}{l}\text { Fall-Cluster) } \\
\text { Fehler }\end{array}$ & 1399,5 & 10 & 139,9 & 0,4225 & 0,934862 \\
\hline
\end{tabular}

Tabelle B-2: Varianzanalytische Ergebnisse für das 5-Sekunden-Intervall:

\begin{tabular}{llllll} 
Effekt & SQ & FG & MSE & F & p \\
\hline Konstante & 689646,8 & 1 & 689646,8 & 1003,749 & 0,000000 \\
Verarbeitungsbedingung & 1975,7 & 2 & 987,9 & 1,438 & 0,245261 \\
Fehler & 42598,4 & 62 & 687,1 & & \\
\hline $\begin{array}{l}\text { Fall-Cluster } \\
\begin{array}{l}\text { Interaktion } \\
\text { (Verarbeitungsbedingung X }\end{array}\end{array}$ & 102553,9 & 5 & 20510,8 & 63,235 & 0,000000 \\
$\begin{array}{l}\text { Fall-Cluster) } \\
\text { Fehler }\end{array}$ & 2591,3 & 10 & 259,1 & 0,799 & 0,629920 \\
\hline & 100550,8 & 310 & 324,4 & & \\
\hline
\end{tabular}

Tabelle B-3: Varianzanalytische Ergebnisse für das 5-Sekunden-Intervall mit ersetzten fehlenden Werten:

\begin{tabular}{llllll} 
Effekt & SQ & FG & MSE & F & p \\
\hline Konstante & 819226,6 & 1 & 819226,6 & 1325,763 & 0,000000 \\
Verarbeitungsbedingung & 2099,9 & 2 & 1049,9 & 1,699 & 0,189900 \\
Fehler & 45726,7 & 74 & 617,9 & & 0,000000 \\
\hline Fall-Cluster & 127602,6 & 5 & 25520,5 & 79,003 & \\
$\begin{array}{l}\text { Interaktion } \\
\text { (Verarbeitungsbedingung X }\end{array}$ & 2047,1 & 10 & 204,7 & 0,634 & 0,784964 \\
$\begin{array}{l}\text { Fall-Cluster) } \\
\text { Fehler }\end{array}$ & 119522,3 & 370 & 323,0 & & \\
\hline
\end{tabular}




\section{Anhang C - Multiple-Choice-Fragen und Antworten in Experiment 2}

(Hinweis: Die korrekten Antworten sind fett gedruckt.)

\section{Kategorisierungsbedingungen}

Deine Aufgabe in diesem Experiment ist es...

... zu entscheiden, wie viele der Warane insgesamt an der Torokrankheit leiden.

... aufgrund der Symptomkonstellation zu entscheiden, welches Medikament die meisten Symptome behebt.

... aufgrund der Symptomenkonstellation zu entscheiden, ob die Torokrankheit bei dem jeweiligen Tier vorliegt, oder ob das betroffene Tier an einer anderen Erkrankung leidet.

... aufgrund der Symptomkonstellation zwischen den 4 Tieren die Symptome zu vergleichen.

Eine Infektion mit den ansonsten eher harmlosen Adeno- und Coronabakterien führt oftmals $\mathrm{zu} \ldots$

... einer eingeschränkten Sicht durch eine Zerstörung der Netzhaut.

... einer Zerstörung der Darmflora und Entzündung der Magenschleimhaut.

... zu starken Schmerzen in Folge einer zerstörten Darmflora.

... Flatulenz, Schwächegefühl, Fieber.

Wenn ein Waran an einer zerstörten Darmflora leidet, führt dies oftmals zu...

... Koliken, Diarrhö und dadurch auch zu Dehydration.

... Koliken, Diarrhö und starken Schmerzen.

... Starken Schmerzen, Erbrechen und dadurch auch zu einer Schädigung der

Speiseröhre.

... einer entzündeten Magenschleimhaut, Diarrhö und Koliken.

Wenn ein Waran an einer entzündeten Magenschleimhaut leidet, führt dies oft auch zu...

... einer zerstörten Darmflora, Diarrhö und Koliken.

... Starken Schmerzen, Erbrechen und dadurch auch zu einer Schädigung der Speiseröhre.

... Koliken, Diarrhö und dadurch auch zu einer Schädigung der Speiseröhre.

... Erbrechen, Diarrhö und starken Schmerzen.

Bei der Torokrankheit können starke Schmerzen verursacht werden durch...

... Koliken.

... einer Zerstörung der Darmflora.

... eine Entzündung der Magenschleimhaut.

... Erbrechen.

Wenn ein Waran durch die Krankheit bedingt brechen muss, führt dies oftmals zu ...

... einer Zerstörung der Darmflora und Entzündung der Magenschleimhaut.

... einer Schädigung der Speiseröhre.

... einer Kolik.

... einer Dehydration. 
Alle Warane, die an der Torokrankheit leiden, haben folgende Symptome ...

... Diarrhö und Dehydration.

... einer erhöhten Anzahl an Adenobakterien oder Coronabakterien.

... einer zerstörten Darmflora und einer entzündeten Magenschleimhaut.

... alle oben genannten Punkte sind falsch. Nicht alle Warane mit Torokrankheit zeigen diese Symptome.

Wenn ein Waran an Diarrhö leidet, führt dies in Folge dessen oft auch zu...

... Erbrechen.

... Dehydration.

... Starken Schmerzen, Erbrechen und dadurch zu einer Schädigung der Speiseröhre.

... Koliken und Dehydration

\section{Interventionsbedingungen}

Deine Aufgabe in diesem Experiment ist es...

... aufgrund der Symptomkonstellation zu entscheiden, welches der beiden in Frage

kommenden Behandlungen alle Symptome behebt.

... aufgrund der Symptomkonstellation zu entscheiden, ob die Tiere krank sind.

... aufgrund der Symptomenkonstellation zu entscheiden, welche der beiden in Frage kommenden Behandlungen effektiver ist und deshalb als erstes durchgeführt werden soll.

... aufgrund der Symptomkonstellation zwischen den 4 Tieren die Symptome zu vergleichen.

Eine Infektion mit den ansonsten eher harmlosen Adeno- und Coronabakterien führt oftmals $\mathrm{zu} \ldots$

... einer eingeschränkten Sicht durch eine Zerstörung der Netzhaut.

... einer Zerstörung der Darmflora und Entzündung der Magenschleimhaut.

... zu starken Schmerzen in Folge einer zerstörten Darmflora.

... Flatulenz, Schwächegefühl, Fieber.

Wenn ein Waran an einer zerstörten Darmflora leidet, führt dies oftmals zu ...

... Koliken, Diarrhö und dadurch auch zu Dehydration.

... Koliken, Diarrhö und starken Schmerzen.

... Starken Schmerzen, Erbrechen und dadurch auch zu einer Schädigung der

Speiseröhre.

... einer entzündeten Magenschleimhaut, Diarrhö und Koliken.

Wenn ein Waran an einer entzündeten Magenschleimhaut leidet, führt dies oft auch zu...

... einer zerstörten Darmflora, Diarrhö und Koliken.

... Starken Schmerzen, Erbrechen und dadurch auch zu einer Schädigung der Speiseröhre.

... Koliken, Diarrhö und dadurch auch zu einer Schädigung der Speiseröhre.

... Erbrechen, Diarrhö und starken Schmerzen. 
Eine der Behandlungen wirkt sehr effektiv auf...

... die Coronabakterien.

... die Adenobakterien.

... eine entzündete Magenschleimhaut.

... eine kaputte Netzhaut.

Eine der Behandlungen wirkt sehr effektiv auf...

... die Adenobakterien

... eine zerstörte Darmflora.

... die Coronabakterien.

... eine kaputte Netzhaut

Alle Warane, die an der Torokrankheit leiden, haben folgende Symptome ...

... Diarrhö und Dehydration.

... einer erhöhten Anzahl an Adenobakterien oder Coronabakterien.

... einer zerstörten Darmflora und einer entzündeten Magenschleimhaut.

... alle oben genannten Punkte sind falsch. Nicht alle Warane mit Torokrankheit zeigen diese Symptome.

Wenn ein Waran an Diarrhö leidet, führt dies in Folge dessen oft auch zu...

... Erbrechen.

... Dehydration.

... Starken Schmerzen, Erbrechen und dadurch zu einer Schädigung der Speiseröhre.

... Koliken und Dehydration. 


\section{Anhang D - Multiple-Choice-Fragen und Antworten in Experiment 3}

(Hinweis: Die korrekten Antworten sind fett gedruckt.)

Deine Aufgabe in diesem Experiment ist es...

... aufgrund der Symptomkonstellation zu entscheiden, welche Therapie die Symptome beheben kann.

... zu entscheiden, ob die Post-Embitterment Disorder in die nächste Version des DSM (Diagnostic and Statistical Manual of Mental Disorders) aufgenommen werden soll.

... aufgrund der Symptomkonstellation zu entscheiden, wie wahrscheinlich die Patienten an einer „Post Embitterment Disorder“" leiden.

... aufgrund der Symptomkonstellation zwischen den Patienten Vergleiche anzustellen.

Treten nach einem Verbitterung auslösenden Ereignis die Symptome gefühlter Kontrollverlust und starke Stressreaktion auf, so führen diese oftmals direkt zu...

... einer verminderten Selbstwirksamkeitserwartung und anhaltenden Symptomen erhöhter Erregung.

... einer wahrgenommenen Handlungsunfähigkeit und Schwierigkeiten ein- oder durchzuschlafen.

... Pessimismus und Reizbarkeit.

... einem Verbitterung auslösenden Ereignis.

Wenn ein Patient an Schwierigkeiten ein- oder durchzuschlafen leidet, führt dies oftmals direkt zu...

... Reizbarkeit.

... anhaltender Müdigkeit am Tage.

... Konzentrationsschwierigkeiten.

... einer Reduzierung der Teilnahme an sozialen Aktivitäten.

Das Gefühl der Entfremdung von Anderen ist oft direkt bedingt durch...

... Pessimismus.

... eine starke Stressreaktion.

... eine Reduzierung der Teilnahme an sozialen Aktivitäten.

... eine anhaltende Müdigkeit am Tage.

Wenn ein Patient an einer starken Stressreaktion leidet, führt diese oftmals zu...

... anhaltenden Symptomen erhöhter Erregung, und dadurch oftmals zu Schwierigkeiten ein-oder durchzuschlafen und oftmals auch zu Reizbarkeit. ... anhaltenden Symptomen erhöhter Erregung, und dadurch oftmals zu einer wahrgenommenen Handlungsunfähigkeit und oftmals auch zu Pessimismus. ... einem Gefühl der Entfremdung von Anderen, und dadurch oftmals zu einer Reduzierung der Teilnahme an sozialen Aktivitäten und oftmals auch zu Konzentrationsschwierigkeiten.

... einer Verminderung der Selbstwirksamkeitserwartungen und dadurch oftmals zu Reizbarkeit und oftmals auch zu Pessimismus. 
Pessimismus ist oft direkt bedingt durch...

... eine wahrgenommene Handlungsunfähigkeit.

... einen gefühlten Kontrollverlust.

... eine Reduzierung der Teilnahme an sozialen Aktivitäten.

... verminderte Selbstwirksamkeitsüberwartungen.

Anhaltende Symptome erhöhter Erregung sind...

... oft bedingt durch einen gefühlten Kontrollverlust und bedingen selbst wiederum oftmals Pessimismus und eine wahrgenommene Handlungsunfähigkeit.

... oft bedingt durch eine starke Stressreaktion und bedingen selbst wiederum oftmals Schwierigkeiten ein- oder durchzuschlafen und Reizbarkeit.

... oft bedingt durch eine Reduzierung der Teilnahme an sozialen Aktivitäten und bedingen selbst wiederum oftmals ein Gefühl der Entfremdung von Anderen.

... oft bedingt durch verminderte Selbstwirksamkeitserwartungen und bedingen selbst wiederum oftmals Schwierigkeiten ein- oder durchzuschlafen.

Im Störungsmodell der Post-Embitterment Disorder führt ein Verbitterung auslösendes Ereignis oftmals direkt zu...

... Pessimismus und wahrgenommener Handlungsunfähigkeit.

... Konzentrationsschwierigkeiten und einem Gefühl der Entfremdung von Anderen.

... Reizbarkeit und dadurch zu einem Gefühl der Entfremdung von Anderen.

... einem gefühlten Kontrollverlust und einer starken Stressreaktion.

Reizbarkeit und Pessimismus...

... bedingen selbst keine weiteren Symptome

... bedingen sich gegenseitig

... werden beide durch verminderte Selbstwirksamkeitserwartungen verursacht

... gehören gar nicht in das Störungsmodell der „Post Embitterment Disorder“

Anhaltende Müdigkeit am Tage...

... ist oftmals bedingt durch anhaltende Symptome erhöhter Erregung und bedingt selbst oftmals eine starke Stressreaktion.

... ist oftmals bedingt durch die Reduzierung der Teilnahme an sozialen Aktivitäten und bedingt selbst oftmals anhaltende Symptome erhöhter Erregung.

... ist oftmals bedingt durch wahrgenommene Handlungsunfähigkeit und bedingt selbst oftmals Konzentrationsschwierigkeiten.

... ist oftmals bedingt durch Schwierigkeiten ein- und durchzuschlafen und bedingt selbst oftmals Konzentrationsschwierigkeiten. 


\section{Anhang E - Instruktionen für Balancierungsbedingung 2 in Experiment 4}

„,Wir kommen nun zum eigentlichen Experiment. Stelle Dir hierzu bitte folgende Situation vor: Du bist Verkäufer bei einer großen internationalen Fondsgesellschaft und u.a. für die Vermarktung neuer Aktienfonds bei Schweizer Kantonalbanken verantwortlich. Damit die neuen Fonds in möglichst vielen der Kantonalbanken den Kunden angeboten werden können (und Du eine möglichst hohe Provision bekommst), ist es wichtig etwas darüber zu wissen, welche der Banken die Produktpolitik der anderen Banken beeinflussen.

In Deinem Vertriebsgebiet gibt es insgesamt neun verschiedene Kantonalbanken (Zürich, Lausanne, Basel, Neuchâtel, Genève, Luzern, Winterthur, St. Gallen und Chur).

- Die Züricher Kantonalbank hat in diesem Bankenverbund normalerweise die Aufgabe, neue Aktienfonds zu bewerten und ggf. in ihr Angebot aufzunehmen. Über die letzten Jahre hat sich gezeigt, dass die Züricher Kantonalbank neue Fonds Deiner Fondsgesellschaft mit einer Wahrscheinlichkeit von 60 Prozent in ihr Sortiment aufnimmt.

- Die anderen Kantonalbanken suchen ebenfalls selbstständig nach neuen für sie relevanten Produkten. Jede dieser Banken führt unabhängig von allen anderen Kantonalbanken ein neues Produkt Deiner Fondsgesellschaft mit einer Wahrscheinlichkeit von 25 Prozent ein.

- Die Produktpolitik der Kantonalbanken ist durch ein komplexes System, welches Du auf der nächsten Seite kennenlernen wirst, miteinander verbunden. Nimmt eine Kantonalbank einen neuen Fonds in ihr Sortiment auf, so werden diejenigen Banken, deren Produktpolitik direkt von dieser Bank beeinflusst wird diesen Fonds mit einer Wahrscheinlichkeit von 80 Prozent ebenfalls in ihr Sortiment aufnehmen.

Die Produktpolitik der einzelnen Kantonalbanken ist folgendermaßen miteinander verbunden (Bitte denke daran, dass die Beeinflussung mit einer Wahrscheinlichkeit von 80 Prozent erfolgt):

- Die Kantonalbank Zürich beeinflusst die Produktpolitik der Kantonalbank Lausanne

- Die Kantonalbank Zürich beeinflusst die Produktpolitik der Kantonalbank Basel

- Die Kantonalbank Lausanne beeinflusst die Produktpolitik der Kantonalbank Genève

- Die Kantonalbank Lausanne beeinflusst die Produktpolitik der Kantonalbank Winterthur

- Die Kantonalbank Genève beeinflusst die Produktpolitik der Kantonalbank Neuchâtel

- Die Kantonalbank Neuchâtel beeinflusst die Produktpolitik der Kantonalbank Luzern

- Die Kantonalbank Basel beeinflusst die Produktpolitik der Kantonalbank Winterthur

- Die Kantonalbank Basel beeinflusst die Produktpolitik der Kantonalbank St. Gallen

- Die Kantonalbank Basel beeinflusst die Produktpolitik der Kantonalbank Chur

- Des Weiteren gibt es keinerlei Einflüsse zwischen den Banken 
Bitte nimm Dir genügend Zeit, um zu verstehen, wie die Banken miteinander vernetzt sind. Es ist für den weiteren Verlauf des Experimentes unerlässlich, dass Du die Zusammenhänge komplett verstanden hast.

Wie Du auf der letzten Folie gesehen hast, wird die Produktpolitik der Kantonalbank Winterthur sowohl von der Kantonalbank Lausanne als auch von der Kantonalbank Basel beeinflusst. Die Ursache hierfür liegt in der geographischen Lage Winterthurs, dass direkt auf der Grenze der Einflussbereiche der Baseler und Lausanner Banken liegt.

Aufgrund der auf den letzten Folien geschilderten Beziehungen und Wahrscheinlichkeiten wird ein neuer Fonds, der von der Züricher Kantonalbank angeboten wird auch von den meisten anderen Kantonalbanken angeboten.

Ein Fonds der zumindest von der Kantonalbank Basel angeboten wird, wird dann mit hoher Wahrscheinlichkeit auch von den Banken in Winterthur, St. Gallen und Chur angeboten.

Ein Fonds der nur von der Kantonalbank Lausanne angeboten wird, wird dann mit hoher Wahrscheinlichkeit auch von den Banken in Genève und Winterthur, wegen Genéve in Neuchâtel und dann am Ende in Luzern angeboten.

Leider hast Du als Fonds-Verkäufer keinen Einfluss auf die Produktpolitik in Zürich, da diese Kantonalbank vollkommen unabhängig über die Fonds entscheidet. Du hast aber großen Einfluss auf die Produktpolitik der Banken in Basel und Lausanne. In den Fällen, in denen die Kantonalbanken Lausanne und Basel einen neuen Fonds nicht anbieten, kommst Du ins Spiel: Du hast die Möglichkeit, einer dieser beiden Kantonalbanken eine äußerst attraktive Gewinnbeteiligung zu gewähren. Dies führt dazu, dass diese Bank den neuen Fonds mit 100\%-iger Wahrscheinlichkeit in ihr Sortiment aufnehmen wird.

Deine Aufgabe wird daher sein, zu entscheiden, welcher der beiden Banken (Lausanne oder Basel) die Gewinnbeteiligung gewährt wird. Wie bereits erwähnt bemisst sich Deine Provision danach, wie viele der neun Kantonalbanken den neuen Fonds anbieten. Hierbei ist einzig und allein die Anzahl der Banken relevant. Daher sollte Deine Entscheidung davon abhängen, wie viele Banken durch deine Entscheidung zusätzlich dazu gebracht werden, den Fonds anzubieten.

So, jetzt hast Du alle Informationen erhalten, die Du benötigst, um als Fondsverkäufer in der Schweiz tätig zu werden. Für die Multiple-Choice-Fragen ist es vor allem wichtig, dass Du folgende Dinge verstanden hast:

- Welche Bank beeinflusst welche andere(n) Bank(en)?

- Wie hoch sind die verschiedenen Wahrscheinlichkeiten dafür, dass

- $\quad$ eine Bank eine andere erfolgreich beeinflusst

- $\quad$ eine Bank einen neuen Fonds unabhängig von der Beeinflussung durch eine andere Bank in ihr Angebot aufnimmt?

- $\quad$ Du selbst eine der Banken davon überzeugst, einen neuen Fonds in ihr Sortiment aufzunehmen?" 


\section{Anhang F - Multiple-Choice-Fragen und Antworten in Experiment 4}

(Hinweis: Die korrekten Antworten sind fett gedruckt.)

\section{Balancierungsbedingung 1}

Wenn die Kantonalbank Zürich einen Fonds anbietet, dann wird dieser in Folge dessen...

... immer auch von den Kantonalbanken Lausanne und Basel angeboten

... mit einer 80\%-igen Wahrscheinlichkeit von der Kantonalbank Neuchâtel angeboten

... mit einer Wahrscheinlichkeit von jeweils $80 \%$ auch von den Kantonalbanken

Lausanne und Basel angeboten

... immer auch von der Kantonalbank Neuchâtel angeboten

Wenn die Kantonalbank Lausanne einen Fonds anbietet, dann wird dieser in Folge dessen...

... mit 80\%-iger Wahrscheinlichkeit gleichzeitig auch in das Angebot aller drei

Kantonalbanken Neuchâtel, Genève und Luzern aufgenommen

... mit 80\%-iger Wahrscheinlichkeit auch in das Angebot der Kantonalbank Zürich aufgenommen

... mit 80\%-iger Wahrscheinlichkeit auch in das Angebot der Kantonalbank Basel aufgenommen

... mit jeweils 80\%-iger Wahrscheinlichkeit auch in das Angebot der Kantonalbanken Neuchâtel, Genève und Luzern aufgenommen

Die Wahrscheinlichkeit dafür, dass die Kantonalbank Zürich einen neuen Fonds in ihr Sortiment aufnimmt beträgt

0 Prozent

25 Prozent

60 Prozent

80 Prozent

Die Wahrscheinlichkeit, dass die Kantonalbank St. Gallen einen neuen Fonds in ihr

Sortiment aufnimmt ohne dabei von einer anderen Bank beeinflusst worden zu sein beträgt

0 Prozent

25 Prozent

60 Prozent

80 Prozent

Das Fondsangebot der Kantonalbank Luzern wird unmittelbar beeinflusst vom

Fondsangebot. .

... der Kantonalbanken Lausanne und Basel

... der Kantonalbanken Winterthur und Chur

... der Kantonalbank Zürich

... der Kantonalbanken Neuchâtel und Genéve 
Ein neuer Fonds, der nicht von der Kantonalbank Winterthur, aber von der Kantonalbank St. Gallen angeboten wird befindet sich mit welcher Wahrscheinlichkeit im Sortiment der Kantonalbank Chur?

$$
\begin{aligned}
& 0 \% \\
& 25 \% \\
& 60 \% \\
& \mathbf{8 0} \%
\end{aligned}
$$

Ein neuer Fonds, der nicht von der Kantonalbank Lausanne, aber von der Kantonalbank Neuchâtel angeboten wird, befindet sich mit welcher Wahrscheinlichkeit im Sortiment der Kantonalbank Genève?
$0 \%$
$25 \%$
$60 \%$
$80 \%$

Befindet sich ein neuer Fonds im Angebot der Kantonalbank Basel, so beeinflusst seine Aufnahme in Basel das Sortiment in Chur...

... auf jeden Fall

... nur indirekt, nämlich wenn die Kantonalbanken Winterthur und St. Gallen den Fonds ebenfalls in ihr Sortiment aufnehmen

... auf keinen Fall

... nur indirekt, nämlich wenn die Kantonalbank St. Gallen den Fonds anbietet, obwohl die Kantonalbank Winterthur ihn nicht im Sortiment hat

Befindet sich ein neuer Fonds im Angebot der Kantonalbank Basel, so ...

... beeinflusst dies nicht die Wahrscheinlichkeit, mit der dieser Fonds auch in Lausanne angeboten wird

... beeinflusst dies die Wahrscheinlichkeit, mit der dieser Fonds auch in Lausanne angeboten wird positiv

... beeinflusst dies die Wahrscheinlichkeit, mit der dieser Fonds auch in Lausanne angeboten wird negativ

... beeinflusst dies die Wahrscheinlichkeit, mit der dieser Fonds auch in Lausanne angeboten wird unvorhersehbar

Befindet sich ein neuer Fonds NICHT im Angebot der Kantonalbank Zürich, so ...

... beträgt die Wahrscheinlichkeit, dass er sich im Sortiment der Kantonalbank

Lausanne oder der Kantonalbank Basel befindet jeweils $\mathbf{2 5 \%}$

... beträgt die Wahrscheinlichkeit, dass er sich im Sortiment der Kantonalbank

Lausanne oder der Kantonalbank Basel befindet jeweils 50\%

... beträgt die Wahrscheinlichkeit, dass er sich im Sortiment der Kantonalbank

Lausanne oder der Kantonalbank Basel befindet jeweils 60\%

... beträgt die Wahrscheinlichkeit, dass er sich im Sortiment der Kantonalbank

Lausanne oder der Kantonalbank Basel befindet jeweils 80\%

Welche Banken kannst Du bezüglich der Aufnahme eines neuen Fonds in ihr Sortiment direkt beeinflussen?

Die Kantonalbanken Neuchâtel und Winterthur

Die Kantonalbanken Lausanne und Genève

Die Kantonalbanken Lausanne und Basel

Die Kantonalbanken Basel und St. Gallen 
Wenn Du eine Kantonalbank hinsichtlich der Aufnahme eines neuen Fonds beeinflusst, so beträgt die Wahrscheinlichkeit, dass diese Bank den Fonds auch tatsächlich in ihr Angebot aufnimmt...

$$
\begin{aligned}
& \ldots 0 \% \\
& \ldots 25 \% \\
& \ldots .80 \% \\
& \ldots .100 \%
\end{aligned}
$$

\section{Balancierungsbedingung 2}

Wenn die Kantonalbank Zürich einen Fonds anbietet, dann wird dieser in Folge dessen... ... immer auch von den Kantonalbanken Basel und Lausanne angeboten ... mit einer 80\%-igen Wahrscheinlichkeit von der Kantonalbank St. Gallen angeboten ...mit einer Wahrscheinlichkeit von jeweils $80 \%$ auch von den Kantonalbanken Basel und Lausanne angeboten

... immer auch von der Kantonalbank St. Gallen angeboten

Wenn die Kantonalbank Basel einen Fonds anbietet, dann wird dieser in Folge dessen...

... mit 80\%-iger Wahrscheinlichkeit gleichzeitig auch in das Angebot aller drei

Kantonalbanken St. Gallen, Chur und Winterthur aufgenommen

... mit 80\%-iger Wahrscheinlichkeit auch in das Angebot der Kantonalbank Zürich aufgenommen

... mit 80\%-iger Wahrscheinlichkeit auch in das Angebot der Kantonalbank Lausanne aufgenommen

... mit jeweils 80\%-iger Wahrscheinlichkeit auch in das Angebot der Kantonalbanken St. Gallen, Chur und Winterthur aufgenommen

Die Wahrscheinlichkeit dafür, dass die Kantonalbank Zürich einen neuen Fonds in ihr Sortiment aufnimmt beträgt

0 Prozent

25 Prozent

60 Prozent

80 Prozent

Die Wahrscheinlichkeit, dass die Kantonalbank Neuchâtel einen neuen Fonds in ihr Sortiment aufnimmt ohne von einer anderen Bank dabei beeinflusst worden zu sein beträgt

0 Prozent

25 Prozent

60 Prozent

80 Prozent

Das Fondsangebot der Kantonalbank Winterthur wird unmittelbar beeinflusst vom Fondsangebot...
... der Kantonalbanken Basel und Lausanne
... der Kantonalbanken Genève und Luzern
... der Kantonalbank Zürich
... der Kantonalbanken St. Gallen und Chur 
Ein neuer Fonds, der nicht von der Kantonalbank Genève, aber von der Kantonalbank Neuchâtel angeboten wird befindet sich mit welcher Wahrscheinlichkeit im Sortiment der Kantonalbank Luzern?

$$
\begin{aligned}
& 0 \% \\
& 25 \% \\
& 60 \% \\
& \mathbf{8 0} \%
\end{aligned}
$$

Ein neuer Fonds, der nicht von der Kantonalbank Basel, aber von der Kantonalbank St. Gallen angeboten wird, befindet sich mit welcher Wahrscheinlichkeit im Sortiment der Kantonalbank Chur?
$0 \%$
$25 \%$
$60 \%$
$80 \%$

Befindet sich ein neuer Fonds im Angebot der Kantonalbank Lausanne, so beeinflusst seine Aufnahme in Lausanne das Sortiment in Luzern...

... auf jeden Fall

... nur indirekt, nämlich wenn die Kantonalbanken Genève und Neuchâtel den Fonds ebenfalls in ihr Sortiment aufnehmen

... auf keinen Fall

... nur indirekt, nämlich wenn die Kantonalbank Neuchâtel den Fonds anbietet, obwohl die Kantonalbank Genève ihn nicht im Sortiment hat

Befindet sich ein neuer Fonds im Angebot der Kantonalbank Lausanne, so ...

... beeinflusst dies nicht die Wahrscheinlichkeit, mit der dieser Fonds auch in Basel angeboten wird

... beeinflusst dies die Wahrscheinlichkeit, mit der dieser Fonds auch in Basel angeboten wird positiv

... beeinflusst dies die Wahrscheinlichkeit, mit der dieser Fonds auch in Basel angeboten wird negativ

... beeinflusst dies die Wahrscheinlichkeit, mit der dieser Fonds auch in Basel angeboten wird unvorhersehbar

Befindet sich ein neuer Fonds NICHT im Angebot der Kantonalbank Zürich, so ...

... beträgt die Wahrscheinlichkeit, dass er sich im Sortiment der Kantonalbank Basel oder der Kantonalbank Lausanne befindet jeweils $\mathbf{2 5 \%}$

... beträgt die Wahrscheinlichkeit, dass er sich im Sortiment der Kantonalbank Basel oder der Kantonalbank Lausanne befindet jeweils $50 \%$

... beträgt die Wahrscheinlichkeit, dass er sich im Sortiment der Kantonalbank Basel oder der Kantonalbank Lausanne befindet jeweils $60 \%$

... beträgt die Wahrscheinlichkeit, dass er sich im Sortiment der Kantonalbank Basel oder der Kantonalbank Lausanne befindet jeweils $80 \%$

Welche Banken kannst Du bezüglich der Aufnahme eines neuen Fonds in ihr Sortiment direkt beeinflussen?

Die Kantonalbanken St. Gallen und Genève

Die Kantonalbanken Basel und Chur

Die Kantonalbanken Basel und Lausanne

Die Kantonalbanken Lausanne und Neuchâtel 
Wenn Du eine Kantonalbank hinsichtlich der Aufnahme eines neuen Fonds beeinflusst, so beträgt die Wahrscheinlichkeit, dass diese Bank den Fonds auch tatsächlich in ihr Angebot aufnimmt...

$$
\begin{aligned}
& \ldots 0 \% \\
& \ldots .25 \% \\
& \ldots .80 \% \\
& \ldots \mathbf{1 0 0 \%}
\end{aligned}
$$




\section{Anhang G - Instruktionen für Balancierungsbedingung 2 in Experiment 5}

„,Wir kommen nun zum eigentlichen Experiment. Stelle Dir hierzu bitte folgende Situation vor: Du bist MitarbeiterIn einer Firma, die neue Schuhe designt und produziert. Einmal im Quartal werden mehrere Prototypen exklusiver neuer Schuhmodelle von der Design-Abteilung deiner Firma entwickelt. Von diesen Prototypen wird jedoch nur ein Modell ausgewählt, das später tatsächlich produziert und verkauft wird. Natürlich soll genau das Modell produziert werden, von dem sich deine Firma den größten Absatz verspricht. Genau hier kommst du ins Spiel, denn deine Aufgabe besteht darin, zu überprüfen, welcher der Prototypen in den meisten der von deiner Firma belieferten Schuhgeschäfte verkauft werden wird. Um diese Aufgabe zu erfüllen, benötigst du einige Informationen, die dir im Folgenden zur Verfügung gestellt werden:

Die Prototypen werden ausschließlich den Filialen von zwei exklusiven Ketten von Schuhgeschäften vorgestellt. Die ersten Filialen der einen Kette wurden in München gegründet, die der anderen in Berlin.

Die Münchner Filiale der ersten Kette eröffnete später drei untergeordnete Filialen in Nürnberg, Frankfurt am Main und Stuttgart. Immer wenn die Münchner Filiale ein neues Schuhmodell in ihr Angebot aufnimmt, sorgt sie dafür, dass auch alle ihr untergeordneten Filialen dieses Modell anbieten, damit die Kunden überall das neue Modell kaufen können. Nimmt hingegen eine der untergeordneten Filialen ein neues Schuhmodell in ihr Angebot auf, so hat diese Filiale keine Möglichkeit dafür zu sorgen, dass das neue Modell auch in München oder in einer der anderen untergeordneten Filialen angeboten wird.

Die Filialen der zweiten Kette sind etwas anders organisiert: Die Berliner Filiale eröffnete lediglich eine untergeordnete Filiale in Leipzig. Die Leipziger Filiale eröffnete später selbst eine eigene untergeordnete Filiale in Hannover. Die Filiale in Hannover eröffnete inzwischen auch eine untergeordnete Filiale in Hamburg. Nimmt eine dieser Filialen einen neuen Schuh in ihr Sortiment auf, so sorgt sie immer dafür, dass auch die ihr direkt untergeordnete Filiale diesen Schuh in ihr Sortiment aufnimmt. Auf die ihr übergeordnete(n) Filiale(n) hat sie allerdings keinen Einfluss.

Insgesamt lässt sich also folgendes Muster feststellen: Eine Filiale sorgt immer und zuverlässig dafür, dass ein von ihr angebotenes neues Schuhmodell in allen der/den ihr direkt untergeordnete(n) Filiale(n) verkauft wird. Die Filiale kann hingegen nicht dafür sorgen, dass ihr übergeordnete Filialen den Schuh auch in ihr Angebot aufnehmen [An dieser Stelle folgte in den Instruktionen eine balancierungsabhängige Darstellung des Kausalsystems vergleichbar mit Abbildung 28].

Nachdem die Designabteilung die vier Prototypen fertiggestellt hat, besuchst du alle Filialen persönlich, um ihnen die Modelle vorzustellen und möglichst viele Filialen dazu zu bewegen, verbindliche Vorbestellungen zu machen. Natürlich hebst Du die Vorzüge aller Prototypen gleichermaßen hervor, da du kein persönliches Interesse an der Produktion eines bestimmten Modells hast, sondern die Filialen unabhängig beraten willst. Im Anschluss an deine Präsentation der vier Prototypen entscheidet sich jede Filiale, welche der Modelle sie verbindlich vorbestellen möchte. Eine Filiale kann frei entscheiden, ob sie keines, eines oder mehrere der Modelle bestellt. Da nur ein Prototyp tatsächlich produziert werden wird, verfallen die Bestellungen für die anderen Modelle. 
Du wirst später Informationen darüber erhalten, welche der Prototypen von welchen Filialen verbindlich vorbestellt wurden. Aus diesen Informationen und dem Wissen darüber, dass die Filialen dafür sorgen, dass ein von ihnen angebotenes Modell auch in der/den ihr untergeordnete(n) Filiale(n) angeboten wird, sollst du dann ermitteln, welches Schuhmodell sich später in den meisten Filialen vorfinden lässt. Hierbei hat es keine Bedeutung, ob ein Schuh von einer über- oder einer untergeordneten Filiale angeboten wird, es geht ausschließlich um die Anzahl der Filialen in denen er letztendlich verkauft wird. Wichtig ist außerdem, dass die Beeinflussung der untergeordneten Filiale(n) erst nach der Entscheidung für die Produktion eines bestimmten Schuhmodells stattfindet, also unabhängig von den Vorbestellungen ist. “ 


\section{Anhang $\mathbf{H}$ - Multiple-Choice-Fragen und Antworten in Experiment 5}

(Hinweis: Die korrekten Antworten sind fett gedruckt.)

\section{Balancierungsbedingung 1}

Deine Aufgabe in diesem Experiment ist es...

... denjenigen Schuh-Prototyp auszuwählen, der von den meisten Filialen vorbestellt wird.

... denjenigen Schuh-Prototyp auszuwählen, der von allen Filialen einer der beiden Ketten vorbestellt wird.

... denjenigen Schuh-Prototyp auszuwählen, der letztendlich in den meisten Filialen angeboten wird.

... den Schuh-Prototyp auszuwählen, bei dem die Differenz zwischen den Filialen, die ihn vorbestellen und den Filialen, die ihn letztendlich anbieten am größten ist.

Wenn die Filiale Berlin einen Schuh vorbestellt, der dann auch tatsächlich produziert wird, dann wird dieser Schuh in der Folge...

... auch in Leipzig, Hamburg und Hannover angeboten.

... entweder in Leipzig oder in Hamburg oder in Hannover angeboten.

... auch in Nürnberg, Frankfurt am Main und Stuttgart angeboten.

$\ldots$ in keiner weiteren Filiale angeboten.

Wenn die Filiale in München einen Schuh vorbestellt, der dann auch tatsächlich produziert wird, dann wird dieser Schuh in der Folge...

... in der Filiale Nürnberg oder der Filiale Frankfurt am Main oder der Filiale Stuttgart angeboten.

... in den Filialen Leipzig, Hamburg und Hannover angeboten.

... unmittelbar in den Filialen Nürnberg, Frankfurt am Main und Stuttgart angeboten.

... in der Filiale Nürnberg, dadurch in der Filiale Frankfurt am Main und dadurch in der Filiale Stuttgart angeboten.

Welcher der folgenden beiden Prototypen würde letztendlich von den meisten Filialen angeboten? Prototyp 1 wird von den Filialen Leipzig und Frankfurt am Main vorbestellt; Prototyp 2 wird von den Filialen Leipzig, Hamburg und Hannover vorbestellt.

Prototyp 1

Prototyp 2

Beide Prototypen würden letztendlich von gleich vielen Filialen angeboten

Ein Schuh-Prototyp, der von der Filiale Frankfurt am Main vorbestellt wurde, würde in der Folge...

... auch in den Filialen Nürnberg und Stuttgart angeboten.

... auch in den Filialen München, Nürnberg und Stuttgart angeboten.

... auch in der Filiale Nürnberg angeboten.

... auch in der Filiale Stuttgart angeboten. 
Ein Schuh-Prototyp, der von der Filiale Hamburg vorbestellt wurde, würde in der Folge...

... von keiner weiteren Filiale angeboten.

... von der Filiale Berlin angeboten.

... von der Filiale Leipzig angeboten.

... von der Filiale Hannover angeboten.

Welche Filialen müssten einen neuen Schuh-Prototypen mindestens vorbestellen, damit er später in allen Filialen angeboten wird?

München, Nürnberg und Leipzig

Berlin und München

Berlin, Hamburg und Frankfurt am Main

Berlin, München, Hannover und Stuttgart

\section{Balancierungsbedingung 2}

Deine Aufgabe in diesem Experiment ist es...

... denjenigen Schuh-Prototyp auszuwählen, der von den meisten Filialen vorbestellt wird.

... denjenigen Schuh-Prototyp auszuwählen, der von allen Filialen einer der beiden Ketten vorbestellt wird.

... denjenigen Schuh-Prototyp auszuwählen, der letztendlich in den meisten Filialen angeboten wird.

... den Schuh-Prototyp auszuwählen, bei dem die Differenz zwischen den Filialen, die ihn vorbestellen und den Filialen, die ihn letztendlich anbieten am größten ist.

Wenn die Filiale München einen Schuh vorbestellt, der dann auch tatsächlich produziert wird, dann wird dieser Schuh in der Folge...

... auch in Nürnberg, Frankfurt am Main und Stuttgart angeboten.

... entweder in Leipzig oder in Hamburg oder in Hannover angeboten.

... auch in Leipzig, Hamburg und Hannover angeboten.

$\ldots$ in keiner weiteren Filiale angeboten.

Wenn die Filiale in Berlin einen Schuh vorbestellt, der dann auch tatsächlich produziert wird, dann wird dieser Schuh in der Folge...

... in der Filiale Leipzig oder der Filiale Hannover oder der Filiale Hamburg angeboten.

... in den Filialen Nürnberg, Frankfurt am Main und Stuttgart angeboten.

... unmittelbar in den Filialen Leipzig, Hannover und Hamburg angeboten.

... in der Filiale Leipzig, dadurch in der Filiale Hannover und dadurch in der Filiale Hamburg angeboten.

Welcher der folgenden beiden Prototypen würde letztendlich von den meisten Filialen angeboten? Prototyp 1 wird von den Filialen Nürnberg und Hannover vorbestellt; Prototyp 2 wird von den Filialen Nürnberg, Frankfurt am Main und Stuttgart vorbestellt.

Prototyp 1

Prototyp 2

Beide Prototypen würden letztendlich von gleich vielen Filialen angeboten 
Ein Schuh-Prototyp, der von der Filiale Hannover vorbestellt wurde, würde in der Folge... ... auch in den Filialen Leipzig und Hamburg angeboten.

... auch in den Filialen Berlin, Leipzig und Hamburg angeboten.

... auch in der Filiale Leipzig angeboten.

... auch in der Filiale Hamburg angeboten.

Ein Schuh-Prototyp, der von der Filiale Stuttgart vorbestellt wurde, würde in der Folge...

... von keiner weiteren Filiale angeboten.

... von der Filiale München angeboten.

... von der Filiale Nürnberg angeboten.

... von der Filiale Frankfurt am Main angeboten.

Welche Filialen müssten einen neuen Schuh-Prototypen mindestens vorbestellen, damit er später in allen Filialen angeboten wird?

München, Nürnberg und Leipzig

Berlin und München

Berlin, Hamburg und Frankfurt am Main

Berlin, München, Hannover und Stuttgart 


\section{Anhang I - Kontraste zwischen den Bedingungen in Experiment 5}

Kontraste für die Differenz der beiden Fall-Cluster zwischen den Verarbeitungsbedingungen in Experiment 5

Tabelle I-1: Kontraste zwischen den Bedingungen in Experiment 5

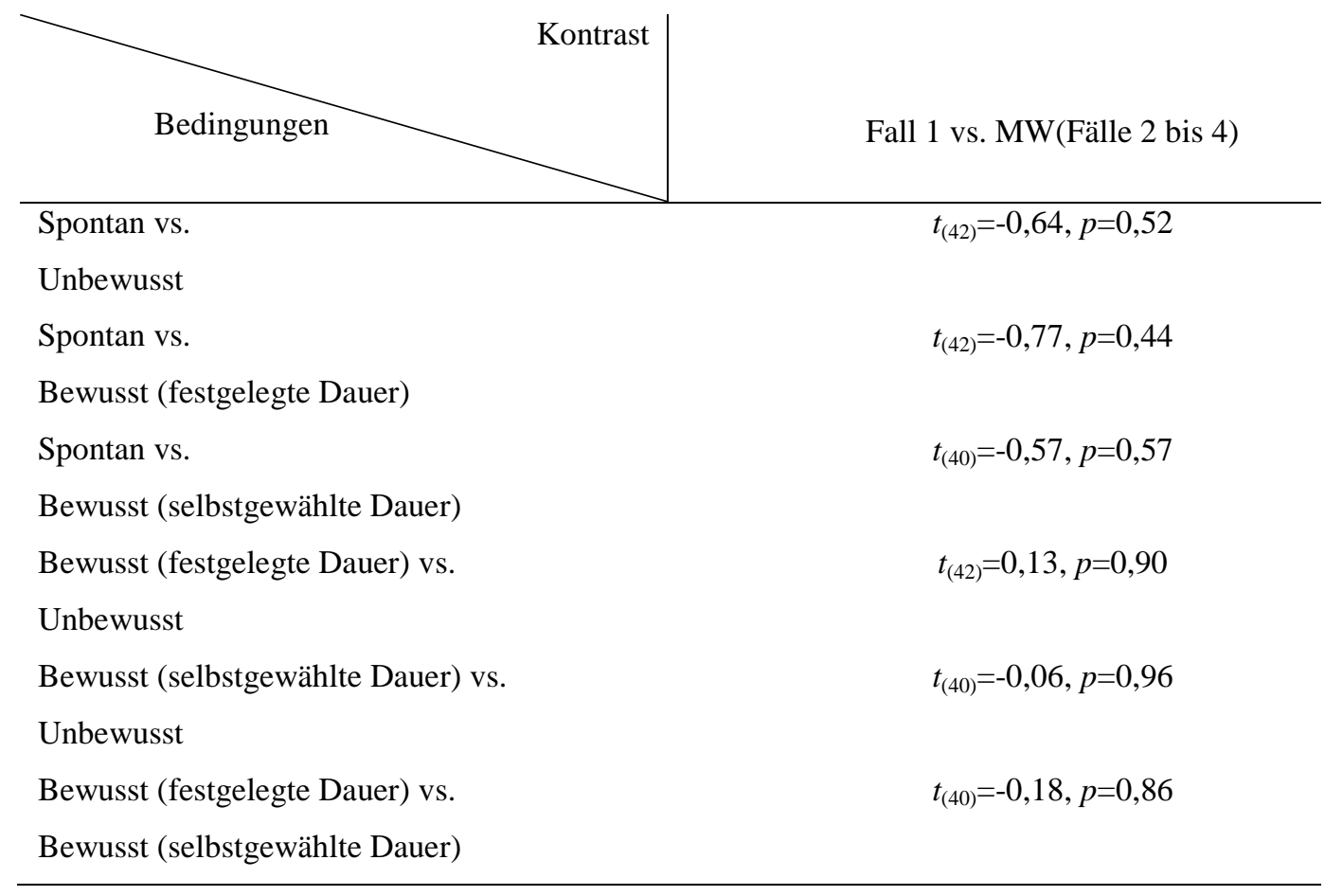




\section{Danksagung}

An dieser Stelle möchte ich mich bei allen Menschen herzlich bedanken, die mir während der Zeit, in der ich an meiner Promotion und dieser Dissertation gearbeitet habe, auf vielfältige Weise unterstützt haben.

Für die Anleitung meiner Promotion danke ich York Hagmayer. Er hatte stets ein offenes Ohr, sei es für neue Ideen oder aber auch neue Probleme, die im Laufe der Zeit entstanden. Darüber hinaus stand er mir stets mit gutem und freundschaftlichen Rat zur Seite und half mir dabei, das Ziel meines Vorhabens nicht aus dem Auge zu verlieren.

Wenn ich in meinem Kämmerlein eine neue Idee ausgebrütet hatte waren es meistens meine Kollegen Jonas, Alex, Ralf und Hans, mit denen ich darüber als erstes diskutierte. Beim Kaffeekochen, im Büro oder einfach auf dem Flur konnte ich so meinen aktuellen Geistesblitz im kritischen Dialog evaluieren und manchmal überlebte sogar noch ein kleiner Funke.

Meinen Freunden innerhalb und außerhalb Göttingens gebührt ebenfalls ein großes Dankeschön dafür, dass Ihr einfach so seid wie Ihr seid! Demnächst werden wir hoffentlich wieder mehr Zeit miteinander verbringen können.

Meinen Eltern kann ich eigentlich gar nicht genug dafür danken, dass sie zu jeder Zeit und in jeder Lebenslage für mich da sind und zu mir stehen. Man kann sich einfach keine besseren Eltern als Euch wünschen!

Zuletzt möchte ich noch meiner Freundin Vanessa von ganzem Herzen danken, die diese für mich nicht immer ganz einfache Zeit mit mir durchlebt und durchlitten hat. Wenn Du lächelst, scheint für mich die Sonne! 


\section{Lebenslauf}

Name: $\quad$ Stefan Mangold

12.12.1977 geboren in Fritzlar

1983-1989 Grundschule in Gudensberg

1988-1994 Dr. Georg-August-Zinn-Gesamtschule in Gudensberg

1994-1997 Friedrich-List-Schule in Kassel; Abitur am 20.06.1997

1997-1998 Zivildienst im Altenzentrum Eben-Ezer in Gudensberg

1998-2004 Betriebswirtschaftslehre-Studium, Georg-August-Universität Göttingen;

Diplom am 05.08.2004

2004-2010 Psychologie-Studium, Georg-August-Universität Göttingen; Diplom am 11.01 .2010

2006-2007 Akademisches Jahr an der University of California, Santa Cruz, USA

seit 04/2010 Promotionsstudium; Abteilung für Kognitionswissenschaften und

Entscheidungspsychologie des Georg-Elias-Müller Instituts für Psychologie an der Georg-August-Universität Göttingen 